\title{
The impact of thermal stress and nutrient availability on the physiology and proteome of symbiotic dinoflagellates
}

\author{
Grace Irene Newson
}

A thesis submitted to the Victoria University of Wellington

in partial fulfilment of the requirements for the degree of

\author{
Master of Science \\ in Marine Biology
}

Victoria University of Wellington, New Zealand

2019

VICTORIA UNIVERSITY OF WELLINGTON

Te Whare Wānanga o te Ūpoko o te Ika a Mãui

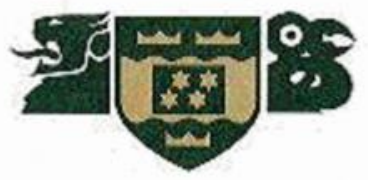




\section{Abstract}

Scleractinian corals, which form the building blocks of tropical reefs, are reliant on a mutualistic symbiosis with phototrophic dinoflagellates of the family Symbiodiniaceae for their metabolic needs and survival. Unfortunately, when subjected to environmental stress this symbiosis can destabilise, culminating in coral bleaching (the loss of symbionts from coral tissue). The most prominent cause of coral bleaching is elevated sea surface temperatures as a result of global warming. However, local stressors such as eutrophication can determine coral reef resilience. Although the physiological responses to temperature and nutrient enrichment are well characterised, the cellular mechanisms underlying these responses are not well understood. This thesis aims to further the understanding of the physiological and cellular responses of Symbiodiniaceae to both thermal stress and nutrient availability.

The Symbiodiniaceae species used in this study was Breviolum minutum (ITS2 ‘type' B1), the homologous symbiont of the model cnidarian Aiptasia. The first objective of this thesis was to compare the physiological response to a rapid versus slow temperature increase, in two strains of the Breviolum minutum (culture IDs: NZ01 and FlAp2), by measuring a range of physiological parameters in cultures exposed to an increase in temperature from 25 to $35^{\circ} \mathrm{C}$, either immediately or over one-week. The physiological measurements taken were: population growth, chlorophyll fluorescence and concentration, photosynthetic and respiratory oxygen flux, and alkaline phosphatase activity (APA). Measurements of chlorophyll fluorescence and oxygen flux demonstrated that NZ01 was able to maintain photosynthetic efficiency and metabolic balance at $35^{\circ} \mathrm{C}$, while FlAp2 was experiencing lethal thermal stress. This divergence in physiological plasticity between strains was emphasised by different heating rates. FlAp2 showed more significant thermal stress at a slower heating rate, exemplified by reduced photosynthetic rates relative to cultures exposed to a rapid temperature increase. Alternately, NZ01 cultures exposed to a slow versus rapid heating rate demonstrated greater thermal acclimation, as alkaline phosphatase activity was elevated, and unlike cultures exposed to a rapid temperature increase, respiration and gross photosynthetic rates were equal to cultures at control temperatures. The intraspecies variability in thermal tolerance demonstrated in this thesis adds to the data supporting the intra-species physiological plasticity of the Symbiodiniaceae family.

The second objective of this thesis was to determine the influence of nutrient supply on the proteomic response to elevated temperature of B. minutum (using the FlAp2 strain). This was achieved by utilising novel proteomics techniques (Liquid chromatography-electrospray ionisation - tandem mass spectrometry, LC-ESI-MS/MS) and various physiological measurements to corroborate trends of 
protein expression (population growth, chlorophyll fluorescence and concentration, photosynthetic and respiratory oxygen flux, and alkaline phosphatase activity). Algal cultures were exposed to either ambient (dissolved inorganic nitrogen: DIN $\sim 1.8 \mu \mathrm{M}$, dissolved inorganic phosphorus: DIP $\sim 0.2 \mu \mathrm{M}$ ), imbalanced (DIN $\sim 26 \mu \mathrm{M}$, DIP $\sim 0.5 \mu \mathrm{M}$ ), or enriched nutrient regimes (DIN $\sim 3 \mu \mathrm{M}$, DIP $\sim 0.55 \mu \mathrm{M}$ ), at either 25 or $34^{\circ} \mathrm{C}$. Although it was hypothesised that there would be an interaction between the influence of temperature and nutrient availability on the Symbiodiniaceae proteome, this was not found. However, separately these environmental stressors had a strong influence on protein abundance. Temperature caused a reduction in photosynthesis proteins, ribosomal proteins, metabolic proteins (Calvin cycle/glycolysis) and proteins involved in biosynthesis, and a relative increased abundance of chaperonin proteins and proteins involved in cellular redox homeostasis.

Interestingly, the Symbiodiniaceae proteome under the ambient and enriched regimes was very similar, while the proteome under the imbalanced nutrient regime was different to these comparatively balanced regimes. This trend highlights the importance of the nitrogen to phosphorus ratio in determining the cellular response of Symbiodiniaceae to nutrient enrichment. Under an imbalanced nutrient regime, there was a down-regulation in photosynthetic and Calvin cycle proteins and an upregulation of proteins involved in protein translation, energy-generating metabolic pathways and storage-product turn-over. Consistent with previous studies, proteomic and physiological data indicated that $B$. minutum might have been experiencing phosphorus deficiency under an imbalanced nutrient regime. However, photochemical efficiency and metabolic balance was maintained, indicating metabolic adaption to the skewed nutrient ratio.

This thesis provides insight into the physiological and cellular response of Symbiodiniaceae to both temperature and nutrients, highlighting potential avenues of research that could be directed to facilitate the knowledge-based management of coral reefs. The intraspecies plasticity demonstrated in chapter two highlights the need to characterise physiological variability within Symbiodiniaceae species, as this could confer an adaptive advantage to the coral holobiont. In conjunction, the proteomics results of chapter three indicate that the relative availability of nitrogen to phosphorus determines the response of Sybiodiniaceae cellular physiology to nutrient availability. This emphasises the importance of determining the threshold of nitrogen to phosphorus that has a negative influence on the coral holobiont, facilitating the setting of ecologically relevant nutrient input limits by coral reef management. 


\section{Acknowledgements}

Firstly, I would like to thank my supervisor Professor Simon Davy. Simon provided me with a huge amount of support and feedback throughout the year and was available to discuss my thesis whenever needed, despite his busy schedule.

I would also like to acknowledge the support I received from the Victoria Masters by Thesis Scholarship, which allowed me to focus on my thesis without the need to also juggle a part time job. I also thank all members of the Davy Lab, all of which have provided me with help and support at one time or another. In particular, I would like to thank Dr Clint Oakley. Clint taught me almost all the laboratory skills I have and showed remarkable patience when answering my endless string of questions. Most significantly Clint trained me in the art of proteomics. Without his support and guidance, I would not have been able to complete the proteomics section of this thesis in such a short time.

I would also like to acknowledge Dr Shaun Wilkinson, who wrote the R code for the permutational analysis of variance of the proteomics data set.

In addition, I would like to thank Sushila Pillai for taking the time to train me on the Olympus FV1000 confocal microscope.

Lastly, I also thank Laura Kelly, who provided me with a protocol to measure the alkaline phosphatase activity of cyanobacteria, which I was then able to adapt for use with Symbiodiniaceae. 


\section{Table of Contents}

Title page

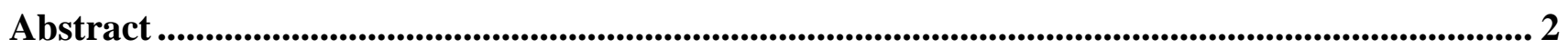

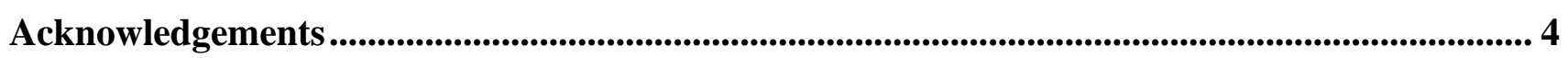

Table of Contents.............................................................................................................................. 5

List of figures .............................................................................................................................................. 8

List of tables .................................................................................................................................... 9

Chapter 1: General Introduction ........................................................................................................... 10

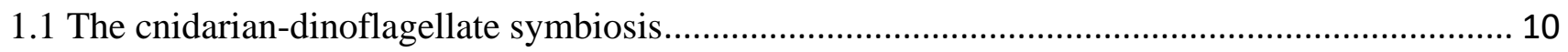

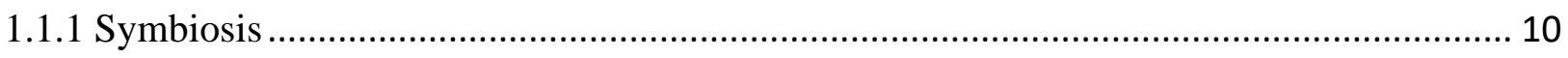

1.1.2 The success of the symbiosis between stony corals and dinoflagellate algae ................... 11

1.1.3 Nutritional exchange and uptake in the cnidarian-dinoflagellate symbiosis ..................... 12

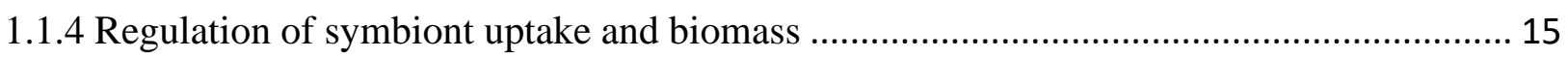

1.2 The breakdown of the cnidarian- dinoflagellate symbiosis...................................................... 16

1.2.1 Cnidarian bleaching ............................................................................................... 16

1.2.2 The oxidative model of cnidarian bleaching ................................................................ 18

1.2.3 Reactive nitrogen species and the activation of cnidarian bleaching ................................ 18

1.3 Symbiodiniaceae photochemistry and photoinhibition ......................................................... 19

1.3.1 The light harvesting pigments of Symbiodiniaceae _....................................................... 19

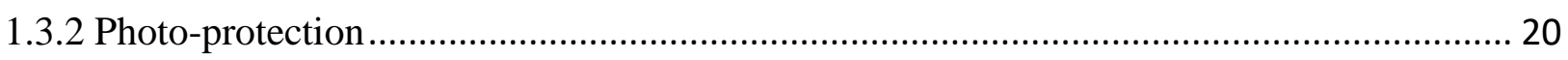

1.3.3 The cellular pathways underlying photosynthetic dysfunction........................................ 21

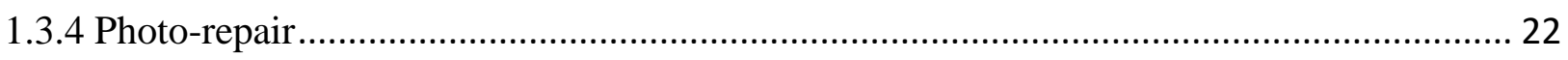

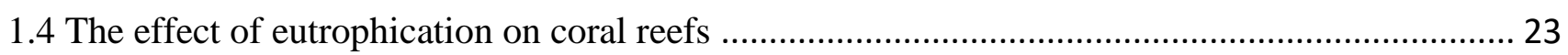

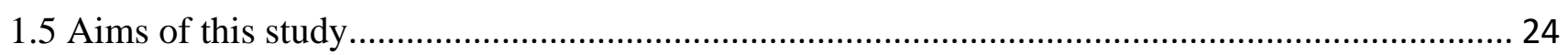

Chapter 2: Characterisation of the physiological response of two Breviolum minutum strains to a slow versus rapid temperature increase .............................................................................................. 26

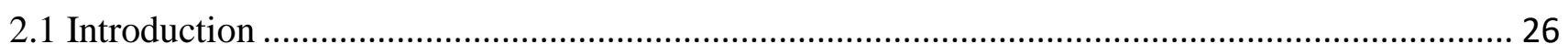

2.1.1 Cnidarian-dinoflagellate symbiosis and the success of reefs .......................................... 26

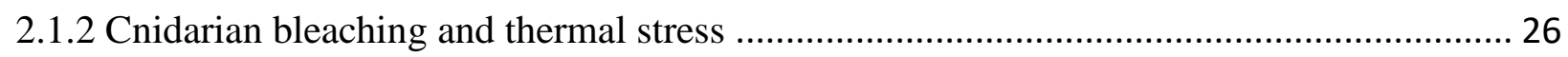

2.1.3 Symbiont diversity and the need to better understand intra-species variability ................ 27

2.1.4 The capacity for Symbiodiniaceae to adapt/ acclimate to climate change.......................... 29

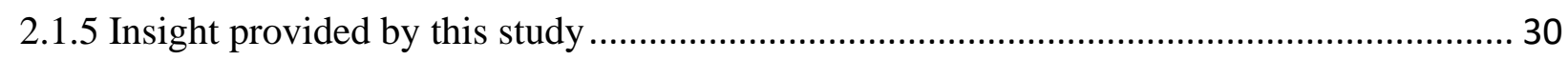

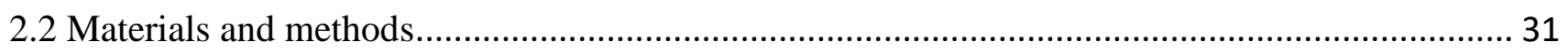

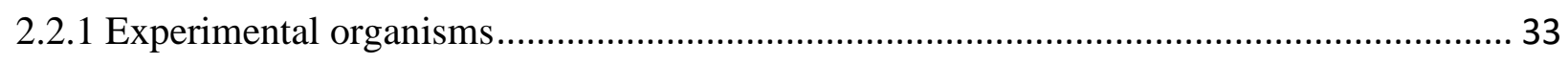

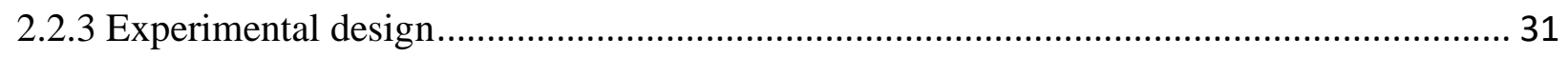

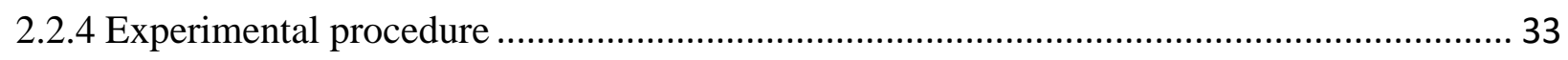




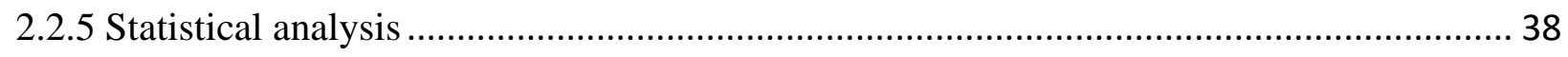

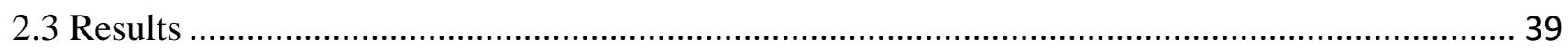

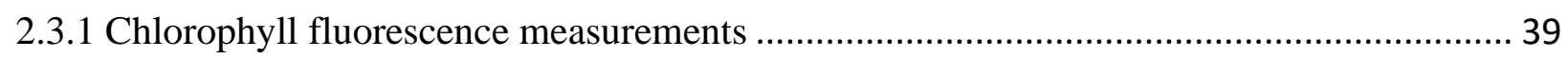

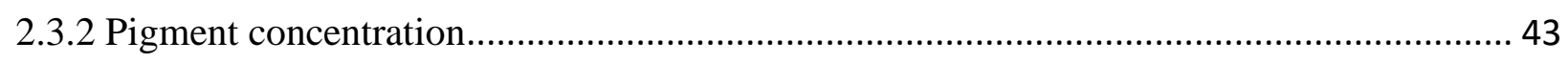

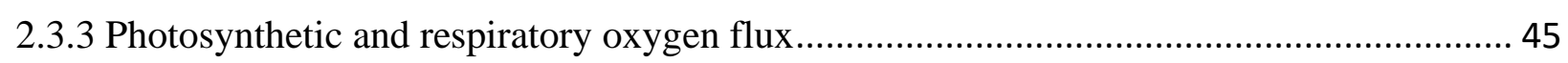

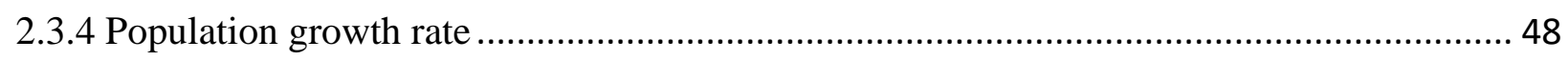

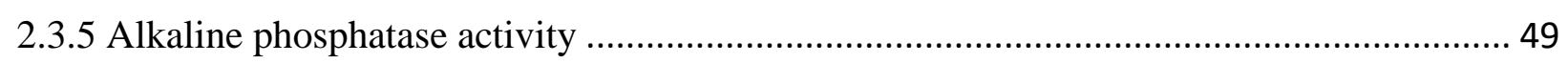

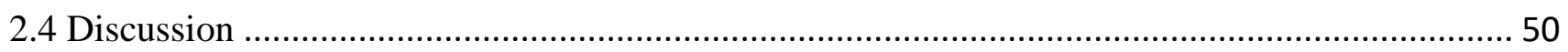

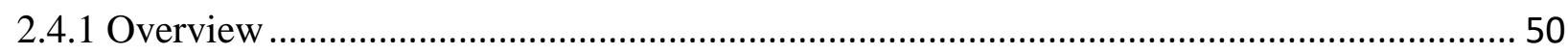

2.4.2 Thermal effects on the photosystems of Breviolum minutum ......................................... 50

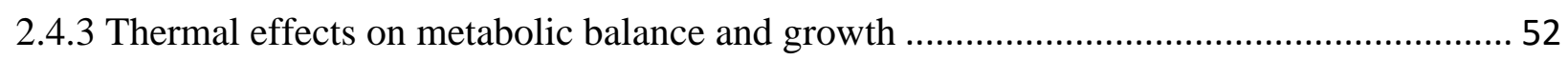

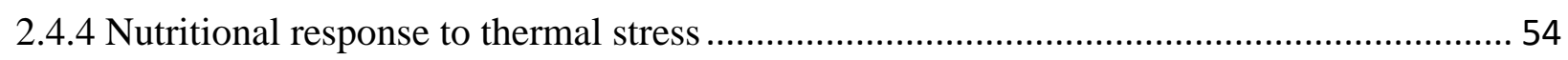

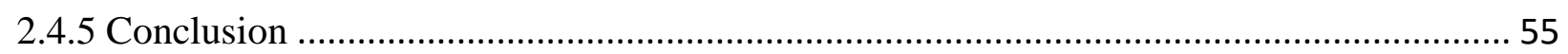

Chapter 3: The link between the nutrient environment and the thermal tolerance of Breviolum

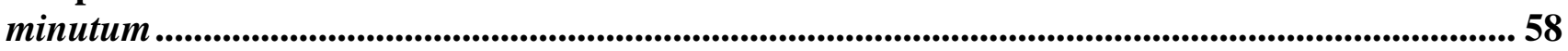

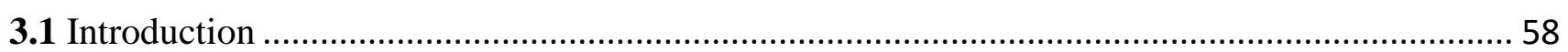

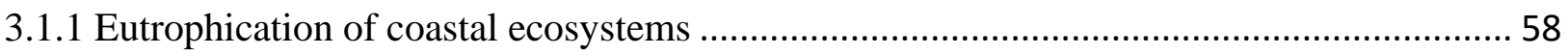

3.1.2 Coral reefs, coral bleaching and eutrophication......................................................... 58

3.1.3 The effect of eutrophication on Symbiodiniaceae ...................................................... 59

3.1.4 'Omics' techniques and their application in increasing our understanding of the cnidarian-

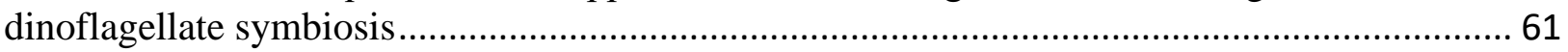

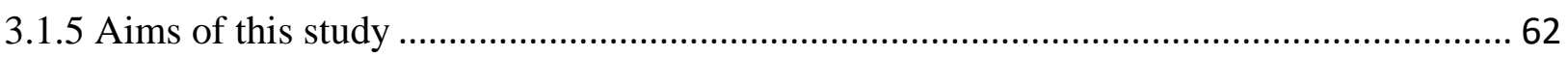

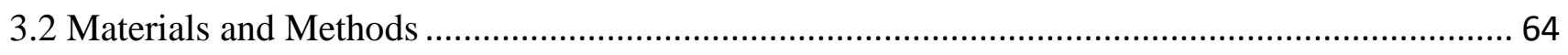

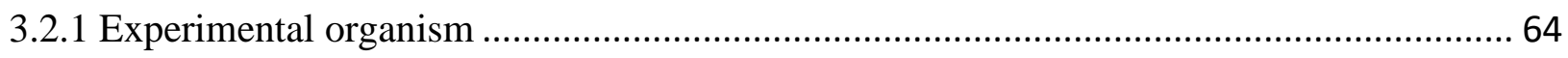

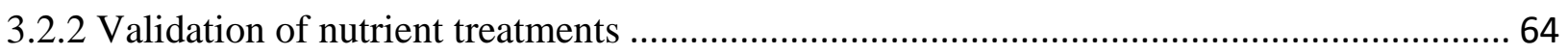

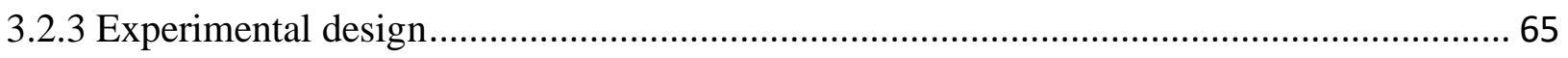

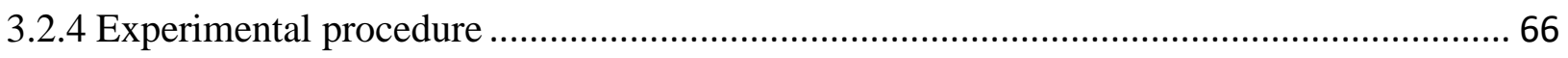

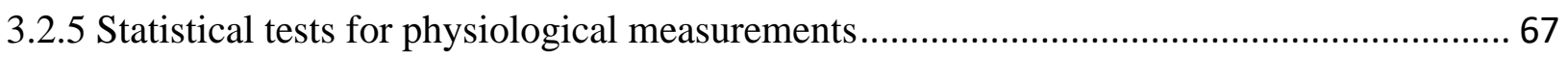

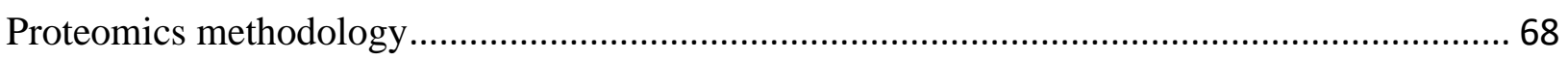

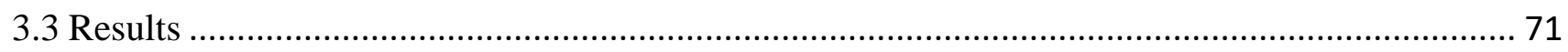

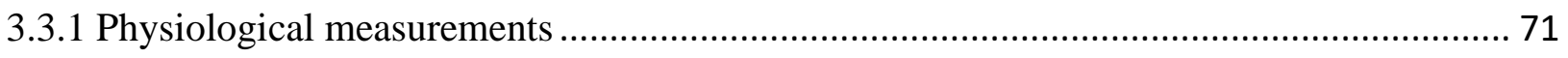

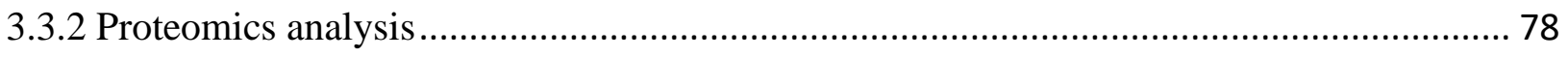

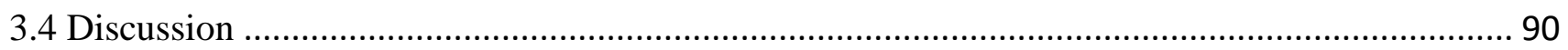

3.4.1 The proteomic response of Breviolum minutum to elevated temperature ......................... 90

3.4.2 The proteomic response of Breviolum minutum to nutrient availability........................... 94

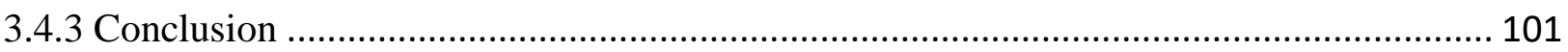


4.1 How might the barrier of host-symbiont specificity be overcome in the context of adaptive bleaching?....

4.2 How might 'omics' technology elucidate the impact of eutrophication on coral reefs at a cellular level and highlight potential avenues for future research? 105

4.3 The results of this thesis in the context of knowledge-based reef management 107

References

Appendix 1: Appendices for Chapter 2

A1.1. Confocal microscope images of stained lipid droplets and starch granules

A1.2. Statistical tests for chapter two physiological measurements

Appendix 2: Appendices for Chapter three

A2.1. Statistical tests for chapter three physiological data A-14

A2.2. Appendices for proteomic analysis A-25

Permutational multivariate analysis of variance. A-25

Tables of differentially abundant proteins between treatments A-25 


\section{List of figures}

Figure 1.1: Microscope image of a cryosection illustrating the symbiosome membrane surrounding Symbiodiniaceae in hospite Exaiptasia pallida ('Aiptasia'). 12

Figure 1.2: Nutritional exchange between partners in the cnidarian-dinoflagellate symbiosis. 15

Figure 1.3: Images of a bleached corals in Hawaii.... 17

Figure 2.1: Diagram depicting the different temperature treatments.

Figure 2.2: Diagram depicting the allocation of biological replicates towards the various physiological measurements preformed in this study

Figure 2.3: NZ01 and FlAp2 strains of Breviolum minutum.

Figure 2.4: Light and dark-adapted chlorophyll fluorescence measurements of two strains of B. minutum at different temperatures.

Figure 2.5: Light and dark-adapted chlorophyll fluorescence measurements of two strains of B. minutum at different temperatures.

Figure 2.6: The concentration of both chlorophyll a and carotenoids, for both NZ01 and FlAp2 cultures.

Figure 2.7: Maximum rates of oxygen evolution and consumption in NZ01 cultures exposed to different temperatures, normalised to cell volume.

Figure 2.8: Maximum rates of oxygen evolution and consumption in FLAp2 cultures exposed to different temperatures, normalised to cell volume.

Figure 2.9: The percentage difference in the cell density of cultures from the beginning of the experimental period to the end of the experimental period.

Figure 2.10: Alkaline phosphatase activity of cultures that were exposed to different temperature treatments.

Figure 3.1: A) Visualisation of experimental design. B) Allocation of biological replicates to the various physiological measurements performed in this study

Figure 3.2: Light and dark-adapted chlorophyll a fluorescence measurements of B. minutum cultures under ambient, imbalanced and enriched nutrient regimes

Figure 3.3: A), (B): The concentration of both chlorophyll a and carotenoids, C) The ratio of carotenoids to chlorophyll a, (D) Alkaline phosphatase activity, (E) The percentage difference in the cell density of cultures from the beginning to the end of the experimental period.

Figure 3.4: Maximum rates of cell-specific photosynthetic oxygen evolution and dark respiratory consumption per hour for cultures exposed to different temperatures and nutrient regimes.....

Figure 3.5: Principal component analysis of label-free quantification protein intensities 78

Figure 3.6: Quantitative proteomic analysis of Symbiodiniaceae cultures at 25 versus $34^{\circ} \mathrm{C}$

Figure 3.7: Quantitative proteomic analysis of Symbiodiniaceae cultures grown under different nutrient regimes. 
Figure 3.8: Counts of proteins that were differentially abundant $(\mathrm{p}<0.05)$ between control $\left(25^{\circ} \mathrm{C}\right)$ and heat-treated $\left(34^{\circ} \mathrm{C}\right)$ B. minutum, grouped by biological process gene ontology terms.

Figure 3.9: Protein interaction network analysis of differentially abundant proteins between 25 and 34 ${ }^{\circ} \mathrm{C}$ in Breviolum minutum.

Figure 3.10: Counts of proteins that were differentially abundant $(p<0.05)$ between Breviolum minutum under the enriched and imbalanced nutrient regimes, grouped by biological process gene ontology terms.

Figure 3.11: Protein interaction network analysis of differentially abundant proteins between an imbalanced and enriched nutrient regime....

Figure 3.12: Counts of proteins that were differentially abundant $(\mathrm{p}<0.05)$ between $B$. minutum under the ambient and imbalanced nutrient regimes, grouped by biological process and function gene ontology terms.

Figure 3.13: Protein interaction network analysis of differentially abundant proteins between an imbalanced and ambient nutrient regime.

Figure 3.14: Conceptual diagram illustrating possible alterations of cellular physiology in B. minutum in response to an imbalanced nutrient regime

\section{List of Tables}

Table 3.1: Nitrogen and phosphorus levels of the ambient, enriched and imbalanced nutrient treatments at the start and end of the experiment.

Table 3.2: Number of differentially abundant proteins between the different thermal and nutrient regimes, based on false discovery rates

Table S1: Differentially abundant proteins between cultures at $25^{\circ} \mathrm{C}$ and $34^{\circ} \mathrm{C}$ based on $\log _{2}$ fold change and a student's t-test.

Table S2: Differentially abundant proteins between the imbalanced and enriched nutrient regime based on $\log _{2}$ fold change and a student's t-test

Table S3: Differentially abundant proteins between the imbalanced and ambient nutrient regime based on $\log _{2}$ fold change and a student's t-test.... 


\section{Chapter 1: General Introduction}

\subsection{The cnidarian-dinoflagellate symbiosis}

\subsubsection{Symbiosis}

Symbiosis is defined as "the living together of two unlike organisms" (De Bary 1879). This association exists on a continuum, ranging from a positive interaction which benefits both partners (mutualistic) to a negative interaction where one organism benefits while the other suffers (parasitic) (Douglas 2008). Precisely where a partnership sits along this continuum is not static, as the symbiotic balance can shift in response to environmental change (Marschner and Dell 1994). The larger partner of the symbiosis is called the host and the smaller partner is termed the symbiont (Smith and Douglas 1987). Symbionts can either be ectosymbiotic and reside on the surface of the host, or endosymbiotic and live inside the host (Smith and Douglas 1987). Symbioses exist across taxa in multiple environments and are often ecologically important. For example, the arbuscular mycorrhizal fungi that live in the root systems of terrestrial plants are estimated to facilitate 75 and $80 \%$ of the phosphorus and nitrogen uptake of these autotrophs, respectively (Van Der Heijden et al. 2008), and hence are central to the maintenance of plant diversity and the ecological functioning of the terrestrial sphere (Van der Heijden et al. 1998, Jeffries et al. 2003). Similarly important are the bacterial symbionts that are commonly found in insects (Ferrari and Vavre 2011). For example, the aphid has a mutualistic symbiosis with the bacterium Buchnera aphidicola, which provides its host with essential amino acids it does not obtain from its diet, receiving carbohydrates and non-essential amino acids in return (Munson et al. 1991, Wilkinson and Douglas 1995). Symbiotic partnerships are also found in aquatic systems, such as between the Hawaiian bobtail squid Euprymna scolopes and the bacterium Vibrio fischeri. In this relationship the luminescence produced by the endosymbiotic bacterium is utilised by the host for counter-illumination, which obscures the silhouette of the host to avoid predators (Jones and Nishiguchi 2004). However, the most well studied marine endosymbionts are single-celled dinoflagellates of the class Dinophyceae (Trench 1997, Gómez 2012) which are present in a range of marine organisms such as sponges, clams, jellyfish, sea anemones and corals (Carlos et al. 1999, Sachs and Wilcox 2006). 


\subsubsection{The success of the symbiosis between stony corals and dinoflagellate algae}

Coral reefs are hot spots of production and biodiversity in otherwise oligotrophic and unproductive tropical waters (Odum and Odum 1955). Although these ecosystems only cover $0.2 \%$ of the ocean's surface, they are estimated to house a third of the world's described marine species (Reaka-Kudla et al. 1997, Reaka-Kudla 2001). In addition, coral reefs can act as a nursery ground for species that do not reside on coral reefs as adults, highlighting the connectivity between coral reef productivity and that of other marine ecosystems (Coker et al. 2014). Not only do coral reefs have immense biological value, but they are also economically important. The ecosystem services of coral reefs are valued at between US\$172 and US\$375 billion per year (Costanza et al. 1997, Moore and Best 2001, Fischlin et al. 2007, Veron et al. 2009). This value is largely built from the tourism and fishing industries associated with coral reefs, in addition to the role that these reefs play in coastal stabilisation by shielding coastlines from tropical storms and cyclones (Johnson et al. 2007). Furthermore, over six million people from small island states are completely reliant on coral reef fisheries for their livelihood, economy and food security (Costanza et al. 1997, Cinner 2014).

Underpinning these ecosystems is the symbiosis between cnidarian stony corals (Order Scleractinia) and dinoflagellate algae, all belonging to the family Symbiodiniaceae (LaJeunesse et al. 2018). These associations are highly successful and have persisted for millions of years. For example, stable isotope analysis of fossilised coral skeletons suggests that the symbiosis between corals and dinoflagellate algae was established 235 million years ago (Stanley and Swart 1995, Muscatine et al. 2005, Stanley Jr 2006). This symbiosis facilitated the formation of productive reef ecosystems in this period, which gave life to the diverse coral reefs we know today (Muscatine et al. 2005). The Symbiodiniaceae family is genetically diverse and has recently been separated into nine phylogenetic groups or genera (LaJeunesse et al. 2018), which used to be known as clades (A-I) (Pochon and Gates 2010, Pochon et al. 2014). Of these clades, A-D are most commonly found to associate with corals, and are now known as the genera; Symbiodinium (Clade A), Breviolum (Clade B), Cladocopium (Clade C), and Durusdinium (Clade D) (Carlos et al. 1999, LaJeunesse 2001, Pochon and Gates 2010, Pochon et al. 2014, LaJeunesse et al. 2018). There is also further diversity within these genera, previously referred to as subcladal phylotypes and now defined as species (LaJeunesse et al. 2018). This genetic diversity is reflected in the differential physiology and resistance to stressful environmental conditions between Symbiodiniaceae species, which in turn may affect how 'valuable' a symbiotic partner they are to their cnidarian host (Silverstein et al. 2015, Swain et al. 2017). In addition, corals are also associated with a diversity of other microorganisms such as bacteria, archaea, and protozoans; collectively this community is termed the coral 'holobiont' (Rohwer et al. 2001). 


\subsubsection{Nutritional exchange and uptake in the cnidarian-dinoflagellate symbiosis}

The foundation of the symbiosis between cnidarians and Symbiodiniaceae is nutritional exchange. These dinoflagellate symbionts are usually found in the gastrodermis of the coral host, surrounded by a series of membranes called the 'symbiosome membrane complex' (Figure 1.1) (Rands et al. 1993, Wakefield and Kempf 2001, Kazandjian et al. 2008). The symbionts are primary producers and provide photosynthetic products such as glucose, glycerol, amino acids, glycoproteins, fatty acids and lipids to their coral host (Whitehead and Douglas 2003, Davy et al. 2012, Matthews et al. 2018) (see Figure 1.2). It is estimated that this photosynthate can support up to $100 \%$ of the coral's metabolic requirements under well-lit conditions (Muscatine et al. 1981, Grottoli et al. 2006, Tremblay et al. 2012), sustaining coral growth, reproduction, and survival (Muscatine et al. 1984, Davies 1991). In return, the dinoflagellates receive coral waste products (ammonium), protection from predation and a stable position in the water column where downwelling light is accessible (Venn et al. 2008, Yellowlees et al. 2008).

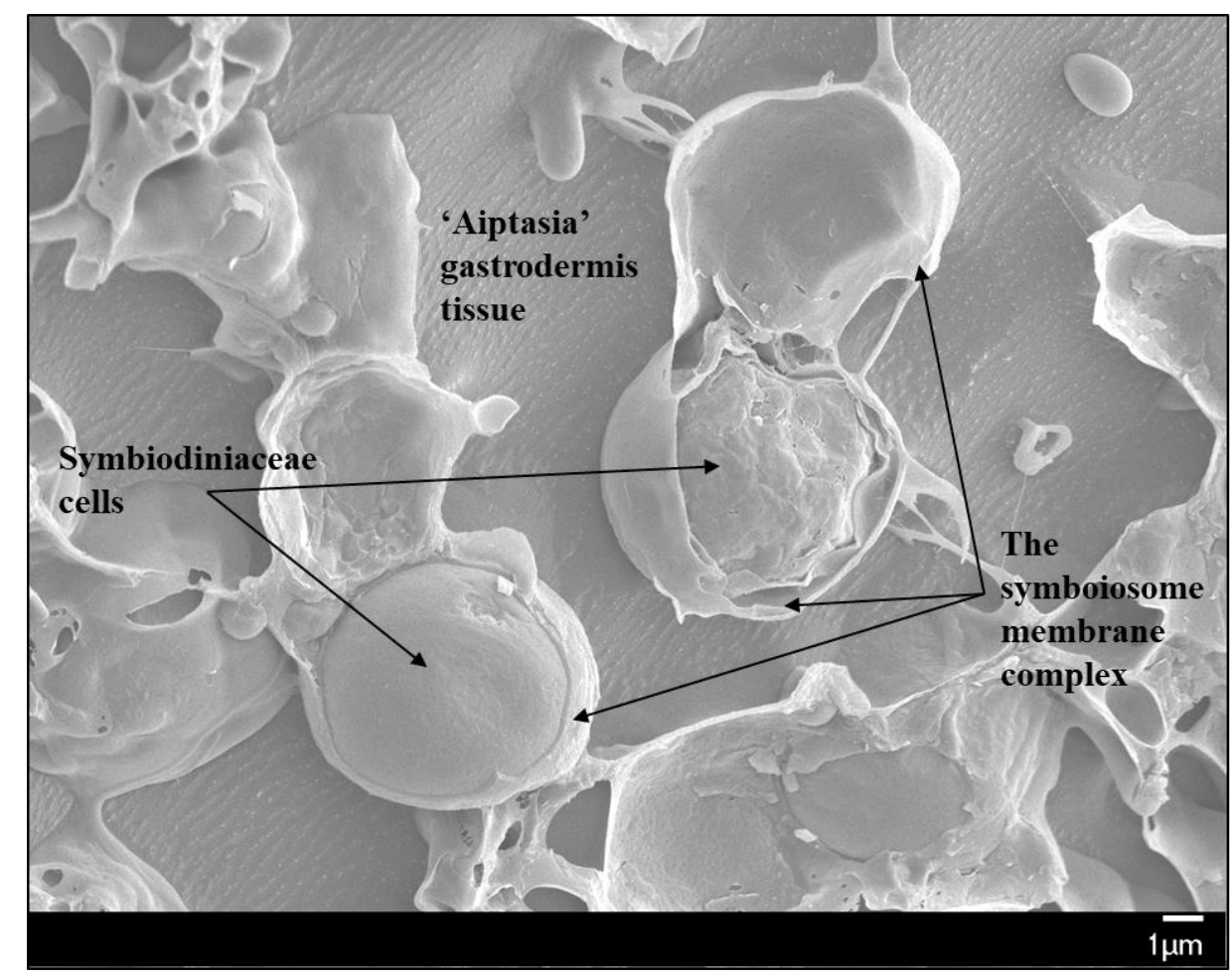

Figure 1.1: Microscope image of a cryosection illustrating the symbiosome membrane surrounding Symbiodiniaceae in hospite Exaiptasia pallida ('Aiptasia'). Photo: A. Mashini. 
As Symbiodiniaceae are endosymbiotic, the host must supply or make available the carbon and nutrients necessary for its survival. For example, $\mathrm{CO}_{2}$ is required for photosynthesis and must be obtained and concentrated to support this physiological function. Carbon exists in seawater largely as $\mathrm{HCO}_{3}{ }^{-}$, which cannot cross cellular membranes due to its charge and so must be converted into $\mathrm{CO}_{2}$ to be assimilated. Both the host (Leggat et al. 2002, Barott et al. 2015b), and its symbionts (Bertucci et al. 2013) have carbonic anhydrases that perform this function (Whitney and Yellowlees 1995). In relation to this, host maintenance of an acidic symbiosome space via the $\mathrm{H}^{+}$-ATPase proton pump may promote the accumulation of carbon dioxide, fuelling photosynthesis (Barott et al. 2015a). Unlike dissolved inorganic carbon the oligotrophic waters of tropical reefs have very low levels of dissolved inorganic nitrogen and phosphorus (DIN and DIP, respectively), which are also necessary for the physiological function of Symbiodiniaceae (Miller and Yellowlees 1989, Smith and Muscatine 1999).

Seawater contains both ammonium and nitrate as sources of inorganic nitrogen, with ammonium thought to be preferred by reef corals (D'elia et al. 1983, Grover et al. 2008). Ammonium is assimilated by the host via the activity of glutamine synthase (GS) and glutamate dehydrogenase (GDH) enzymes (Miller and Yellowlees 1989, Wang and Douglas 1998). Alternatively, ammonium is taken up by the symbiont by GS and / or glutamine 2-oxoglutarate aminotransferase (GOGAT) enzymes (Swanson and Hoegh-Guldberg 1998, Roberts et al. 1999, Roberts et al. 2001, Pernice et al. 2012). Nitrate, on the other hand, can only be assimilated by the symbiont, via an enzymatic cycle involving nitrate reductase and reduced ferredoxins $(\mathrm{Fd})$ from the electron transport chain of photosystem II (Tanaka et al. 2006, Kopp et al. 2015). Nitrogen can be recycled between the members of this symbiosis, as catabolism of nitrogenous compounds by the host generates ammonium, which can in turn be taken up by the symbionts and utilised to produce amino acids (Wang and Douglas 1999, Davy et al. 2012, Pernice et al. 2012). This allows the recycling and retention of nitrogen within the holobiont (see Figure 1.2) and contributes to the success of coral reefs in the oligotrophic tropical waters in which they reside (Muscatine and Porter 1977, Wang and Douglas 1998).

In comparison, phosphorus can be obtained in either its organic or inorganic forms (Björkman and Karl 1994). Dissolved organic phosphorus (DOP) and particulate organic phosphorus (POP) are likely to be sourced via host heterotrophy but must be converted into DIP in order to be assimilated (FerrierPagès et al. 2016). Evidence suggests that DIP is absorbed and concentrated from surrounding seawater by carrier-mediated active transport in the host and the symbiont (Jackson and Yellowlees 1990, Godinot et al. 2009, Ferrier-Pagès et al. 2016). In addition, phosphate uptake has been observed to be inhibited in the absence of sodium, suggesting the involvement of a sodium phosphate 
symporter in phosphate uptake across the host membrane (Godinot et al. 2011b). However, the specific enzymes that mediate phosphate uptake in the symbiont and host are not yet characterised.

Both nitrogen and phosphorus are essential for symbiont growth. Specifically, Symbiodiniaceae require a ratio of one nitrogen atom for every seven carbon atoms in order to proliferate (Dubinsky and Berman-Frank 2001). Any carbon in addition to this ratio may be respired, stored or translocated to the host (Dubinsky and Berman-Frank 2001). As cell division rates are higher when Symbiodiniaceae are grown in culture relative to in hospite (Miller and Yellowlees 1989, Muscatine et al. 1989b, Smith and Muscatine 1999, Davy et al. 2012), it is thought that the host controls the symbionts' intracellular nutrient environment, forcing uncoupled photosynthetic and growth rates and ensuring maximal translocation of photosynthetic products (Cook et al. 1988, Falkowski et al. 1993, Smith and Muscatine 1999, Kopp et al. 2013, Jiang et al. 2014). However, the capacity of the coral host to control the availability of nutrients to its symbionts may still be limited, as the symbionts have been observed to react to changes in the nutrient environment outside the coral host (Stambler et al. 1991, Fabricius 2005, D'Angelo and Wiedenmann 2014). In addition to this mechanism, it is also hypothesised that the host promotes photosynthate release via a signalling compound coined 'the host release factor' (HRF). However, throughout the literature there has been debate as to whether HRF truly operates in the intact association, and if so what its identity is (Muscatine et al. 1972, Gates et al. 1995, Wang and Douglas 1997, Withers et al. 1998, Cook and Davy 2001, Grant et al. 2001, Grant et al. 2006). The maintenance of carbon translocation is therefore inherent to symbiosis stability (Muscatine et al. 1981), though, various other mechanisms likely also play a role, such as interpartner signalling and alternate mechanisms of symbiont biomass regulation (McAuley and Smith 1982, Smith and Muscatine 1999, Davy et al. 2012). 


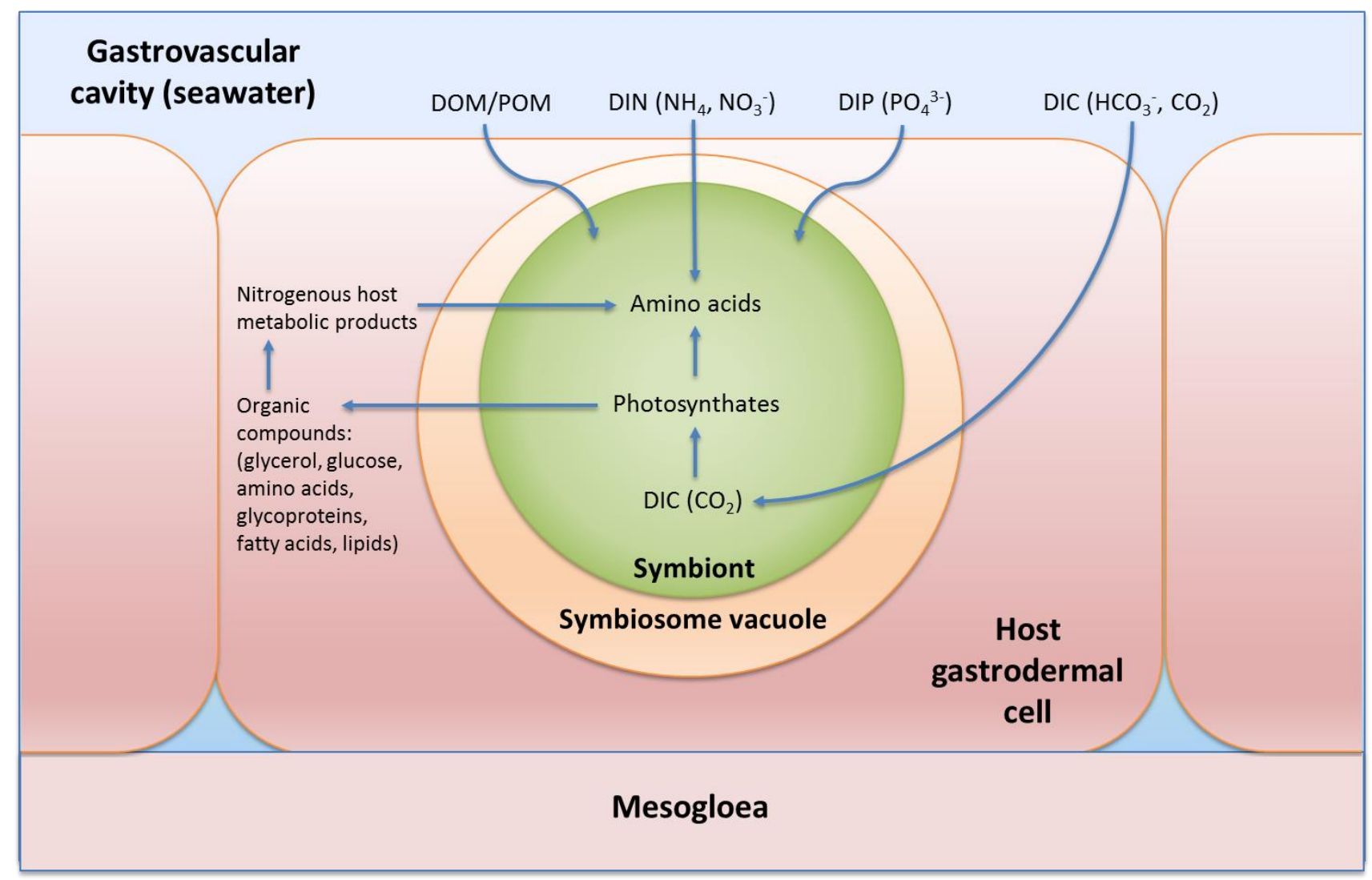

Figure 1.2: Nutritional exchange between partners in the cnidarian-dinoflagellate symbiosis. $\mathrm{POM}=$ Particulate Organic Matter; DOM= Dissolved Organic Matter; DIN= Dissolved Organic Nitrogen; DIP= Dissolved Organic Phosphate; DIC= Dissolved Organic Carbon. (After Davy et al., 2012).

\subsubsection{Regulation of symbiont uptake and biomass}

Various cellular mechanisms control the establishment of the cnidarian-dinoflagellate symbiosis. Cnidarians can acquire symbionts either by horizontal or vertical transmission. Horizontal transmission is where coral larvae or newly metamorphosed primary polyps acquire dinoflagellate symbionts from their surrounding environment (the seawater) (Van Oppen 2001, Hirose et al. 2008), whereas vertical transmission is when symbionts are transferred from the parent coral to developing eggs (Padilla-Gamiño et al. 2012). The majority of host-symbiont combinations are very specific, however a few coral species are more generalist and can form a symbiosis with a variety of symbiont types (Fabina et al. 2012, Silverstein et al. 2012). Upon symbiosis establishment, molecular signalling and cell-surface interactions allows the host to identify compatible symbiont species, and differentiate symbionts from potential pathogens (McAuley and Smith 1982, Bay et al. 2011, Davy et al. 2012). If a Symbiodiniaceae cell is not compatible with the host, its innate immune system will react to remove 
the symbiont, hypothesised to be achieved via autophagy (cell degradation) (Levine and Klionsky 2004, Davy et al. 2012) or apoptosis (cell death) (Dunn and Weis 2009, Davy et al. 2012).

A key factor underlying the persistence of this partnership once established is the demographic control of the Symbiodiniaceae population. This ensures that the symbiont population does not outgrow its coral host and become a carbon sink instead of a carbon source, representing a shift from mutualism towards parasitism along the continuum of a symbiosis (Muscatine et al. 1989a, Muscatine et al. 1989b, Muscatine et al. 1998). The coral host can control symbiont populations under normal environmental conditions by a number of mechanisms. Host limitation of the symbionts' nutrient environment and the HRF, mentioned above as mechanisms of ensuring the release of photosythates to the host, may also play a role in symbiont population regulation by limiting resource availability and restricting mitosis, arresting the Symbiodiniaceae cell cycle in the $\mathrm{G}_{1}$ phase (Falkowski et al. 1993, Smith and Muscatine 1999, Davy et al. 2012). However, there is also evidence that the symbiont cell cycle may be limited by a host-derived factor other than inorganic nutrients. In support of this, Smith and Muscatine (1999) reported that the $G_{1}$ phase of the symbionts was much longer in hospite than in culture, with similar results seen under nutrient-enriched conditions. The mechanism behind this pathway has yet to be determined.

In addition, if symbiont populations become too large the host can actively remove symbionts by degrading symbionts in situ (Titlyanov et al. 1996, Titlyanov et al. 2004), expulsion (HoeghGuldberg et al. 1987, Stimson and Kinzie 1991, McCloskey et al. 1996, Titlyanov et al. 1996, Titlyanov et al. 2004), or both in concert (Fujise et al. 2014). There is evidence that this process is non-random, as preferential expulsion of dividing symbionts has been observed (Baghdasarian and Muscatine 2000).

\subsection{The breakdown of the cnidarian- dinoflagellate symbiosis}

\subsubsection{Cnidarian bleaching}

The presence of Symbiodiniaceae within host cells gives coral tissue a golden-brown colour. Healthy corals contain millions of symbionts per square centimetre of tissue (Weis 2008). However, when an environmental perturbation occurs, symbionts themselves can die due to stress and/or they are expelled from coral tissue, causing the coral to lose its pigmentation (see Figure 1.3) (Douglas 2003, Weis 2008). This phenomenon is called 'coral bleaching'. Bleached corals suffer nutrient deprivation, increased susceptibility to disease, and mortality if a symbiosis cannot be re-established (Goreau and 
Macfarlane 1990, McClanahan 2004). Both members of this symbiosis have evolved protective mechanisms to prevent bleaching damage, including fluorescent proteins and mycosporine-like amino acids to absorb high levels of radiation, production of heat shock proteins to mitigate protein dissociation under high temperatures, and antioxidant systems to neutralise reactive species (reviewed in Baird et al., 2009). It is when these defence systems become over-whelmed due to stress that corals begin to bleach (Weis 2008).

Bleaching is provoked by increased salinity, sedimentation, eutrophication, solar radiation (visible light + UV), or most significantly sea surface temperatures (Coles and Brown 2003, D’Angelo and Wiedenmann 2014). Global warming as a result of the accumulation of anthropogenically-produced greenhouse gases in the Earth's atmosphere is labelled as the primary cause of the global increase in coral bleaching over the last century (Hughes et al. 2003, Hoegh-Guldberg et al. 2007). At the current rate of global warming, it is projected that coral reefs worldwide will be subjected to annual bleaching events by 2040 (Van Hooidonk et al. 2013), which is predicted to result in global mass mortality of coral reefs within decades (Baker et al. 2008, Van Hooidonk et al. 2013, Donner et al. 2017). In conjunction, increasing levels of atmospheric $\mathrm{CO}_{2}$ are resulting in ocean acidification, which can compromise carbonate accretion and thus threaten the framework of coral reef ecosystems (HoeghGuldberg et al. 2007, De'ath et al. 2009). This degradation of coral reef habitats can have cascading negative effects throughout the reef community (De'ath et al. 2012).

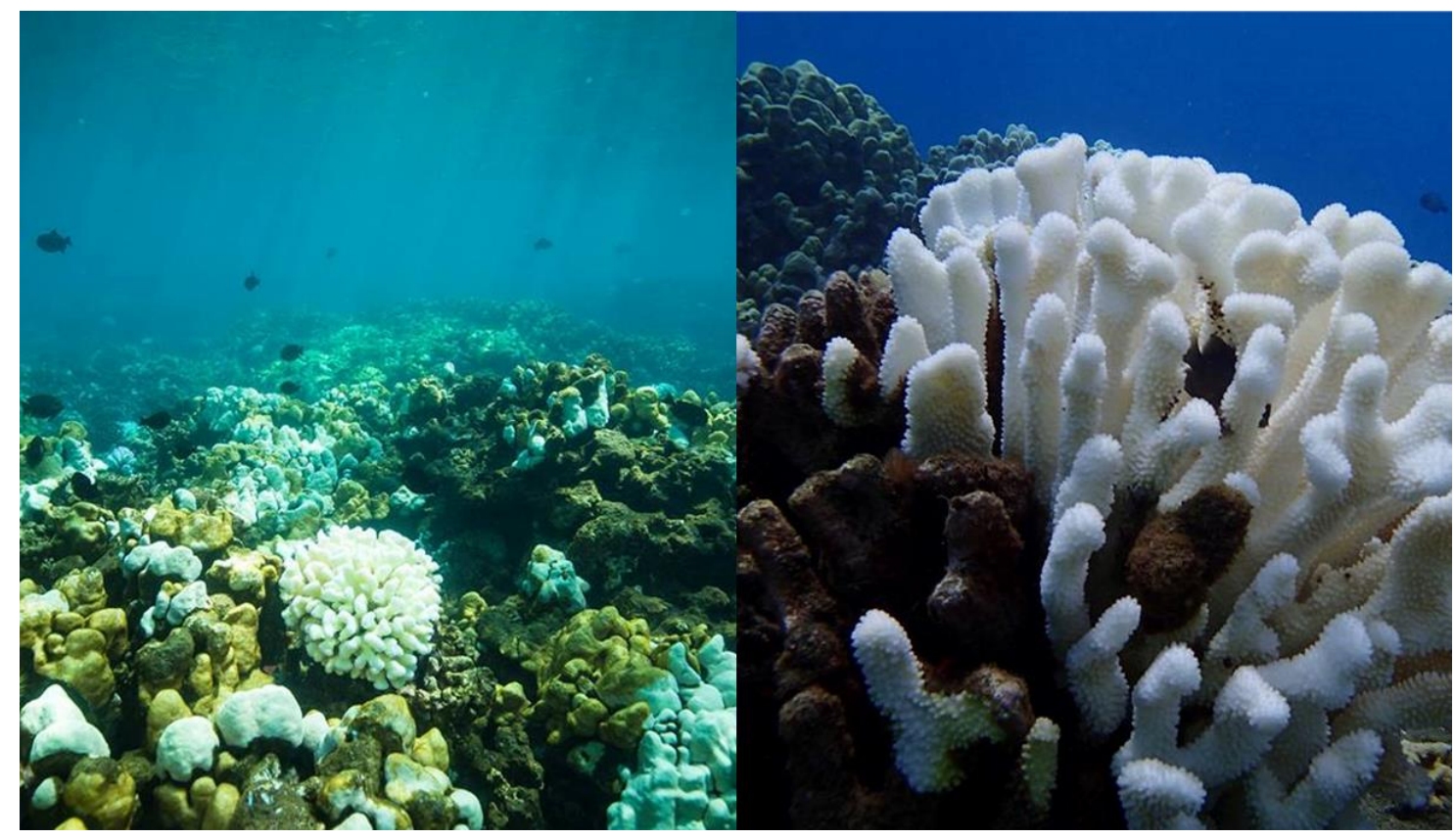

Figure 1.3: Images of a bleached corals in Hawaii (Photo: G.J. Williams). 


\subsubsection{The oxidative model of cnidarian bleaching}

In the face of global coral reef decline, it is essential to understand the mechanism of coral bleaching. However, while the environmental stressors that trigger bleaching are established, the cellular and molecular pathways that result in symbiont loss are not well understood (Weis 2008). It is generally accepted that the build-up of reactive oxygen species (ROS) and the resultant oxidative stress, sparks the cellular cascade of cnidarian bleaching (Weis 2008, Rehman et al. 2016, Oakley and Davy 2018). Environmental stressors such as elevated irradiance and sea surface temperatures (Hoegh-Guldberg et al. 2007, Hughes et al. 2017) can cause the dysfunction of Symbiodiniaceae's photosynthetic apparatus, through overexcitaion, photoinhibtion and sink limitation (described in detail in Section 1.3.3). These processes lead to the formation of reactive oxygen species (ROS), such as singlet oxygen $\left(\mathrm{IO}_{2}\right)$ and superoxide $\left(\mathrm{O}_{2}^{-}\right)$(Lesser 2006, Warner and Suggett 2016), which may subsequently leak into host tissue (Saragosti et al. 2010, Rehman et al. 2016). While earlier studies focused on the symbiont as the primary producer of ROS, more recent literature has shifted focus to the role of the host in the initiation of the bleaching cascade (Dunn et al. 2012, Paxton et al. 2013, Tolleter et al. 2013, Krueger et al. 2015, Lutz et al. 2015). Thermally-induced damage to the host mitochondrion and endoplasmic reticulum can also generate high levels of ROS (Dunn et al. 2012, Lutz et al. 2015, Oakley et al. 2017, Ruiz-Jones and Palumbi 2017). ROS can oxidise membranes, denature proteins and damage nucleic acids, causing significant structural and functional cellular damage in both the symbiont and host (Lesser 2006). Both partners produce antioxidant enzymes which neutralise these reactive species, such as ascorbate peroxidase (APX), catalase and different forms of superoxide dismutase (SOD) (Richier et al. 2005, Lesser 2006, Merle et al. 2007). However, as ROS production increases under stressful conditions, these antioxidant defence systems become overwhelmed (Lesser 1996, Franklin et al. 2004, Lesser 2006, Weis 2008). The build-up of ROS and other reactive species such as nitrogen radicals (discussed in Section 1.2.3), is thought to activate the host's innate immune system (Detournay et al. 2012, Mydlarz et al. 2016). The resulting immune response acts to remove symbionts from host tissue by several mechanisms, such as exocytosis, symbiont digestion, and most prominently host cell apoptosis, or programmed cell death (Weis 2008, Tchernov et al. 2011).

\subsubsection{Reactive nitrogen species and the activation of cnidarian bleaching}

In addition to ROS, reactive nitrogen species (RNS) have also been linked to the cnidarian bleaching response (Weis 2008). Nitric oxide (NO) has been identified as a key cell-signalling and antimicrobial 
molecule, playing a central role in the regulation of microbial endosymbioses (Fang 2004, Wang and Ruby 2011). There is evidence of NO production in response to thermal stress by both the cnidarian host (Perez and Weis 2006, Hawkins and Davy 2013, Hawkins et al. 2014), and its symbiotic dinoflagellates (Trapido-Rosenthal et al. 2001, Bouchard and Yamasaki 2008, Hawkins and Davy 2012, 2013), suggesting that this molecule contributes to the mediation of the symbiosis as well. The model symbiotic anemone, Exaiptasia pallida ('Aiptasia'), has even been induced to bleach by NO addition under ambient temperatures (Perez and Weis 2006). It is hypothesised that NO production is linked to the activation of the host's innate immune system (Perez and Weis 2006, Weis 2008, Detournay et al. 2012). With regards to this, NO synthesis has been correlated with the activation and regulation of host apoptosis-like pathways (Radi et al. 2002, Lesser and Farrell 2004, Perez and Weis 2006, Snyder et al. 2009, Man and Kanneganti 2016), connecting increased NO levels with the bleaching response (Hawkins and Davy 2013).

\subsection{Symbiodiniaceae photochemistry and photoinhibition}

\subsubsection{The light harvesting pigments of Symbiodiniaceae}

Members of the Symbiodiniaceae contain chlorophylls $a$ and $c_{2}$, but unlike higher plants their primary light-harvesting pigment is peridinin, which has a higher absorbance of green and blue light when compared to chlorophyll (Brown et al. 1999). These pigments combine to form the chlorophyll $a$ chlorophyll c2-peridinin-protein-complex (acpPC), which is found within the membrane of the chloroplast (Niedzwiedzki et al. 2014). These complexes absorb light energy, which is then passed on through the electron transport chains of photosystems II and I to ferredoxin-(NADP ${ }^{+}$) reductase which accepts electrons and transfers them to the Calvin cycle to be used to fix carbon (Warner and Suggett 2016). The efficiency of the photosynthetic electron transport chain is related to the photosynthetic productivity and health (Genty et al. 1989). This can be measured by chlorophyll induction fluorometry (Warner et al. 1996). Specifically, the effective quantum yield measurements taken in the light give an indication of the amount of energy used for photochemistry by photosystem II under steady state conditions. Alternately, the maximum quantum yield measurements taken in darkness provide information on the number of reaction centres available for photosynthetic reaction. This is useful information, as when plants experience stress they downregulate photosystem II by reducing the number of open reaction centres, which protects against damage by overexcitation (Hill et al. 2005, Gururani et al. 2015, Warner and Suggett 2016). Generally, fluorescence values of 0.5 and above are considered within the healthy range, with anything below 0.4 indicating photoinhibition 
(Gorbunov et al. 2001). This metric has been linked to cnidarian bleaching, with evidence of a decline in quantum yield before the loss of algal symbionts from host tissue (Hill et al. 2004, Hill et al. 2005, Warner and Suggett 2016).

\subsubsection{Photo-protection}

Like higher plants, Symbiodiniaceae have various regulatory mechanisms to ensure that when light is absorbed in excess of what is needed for $\mathrm{CO}_{2}$ fixation, the photosynthetic apparatus does not become over-excited, so causing damage (Warner and Suggett 2016). For example, photosystem overload can be avoided by diverting light energy away from the photosystems or reducing the amount of light absorbed originally. This can be achieved by reducing the number of photosynthetic units or lightharvesting pigments, thus reducing the amount light energy absorbed by the alga (Falkowski and Raven 2007). Alternately, light energy can be redirected away from regular photosynthetic linear electron transport to alternate electron flow (AEF) pathways to reduce over-excitation (Roberty et al. 2014). AEF pathways include: cyclic electron flow around PSII, the Mehler reaction, chlororespiration, photorespiration, and mitorespiration (Asada 1999, Badger et al. 2000, Cardol et al. 2008, Cardol et al. 2011, Roberty et al. 2014). The interaction and precise roles of these AEF's are not fully elucidated, however it is well accepted that CEF activity acts to build a proton gradient across the thylakoid membrane which activates $\mathrm{qE}$, a form of non-photochemical quenching (NPQ) (Warner et al. 1996, Shikanai 2014, Aihara et al. 2016). NPQ is the dissipation of light energy as heat. There are three pathways of NPQ; energy dependent quenching (qE), state transitions (qT), and photoinhibitory processes $(\mathrm{qI})$.

The quenching of light energy by qE is linked to the xanthophyll cycle (Brown et al. 1999, Kanazawa et al. 2014). An increased pH gradient across the thylakoid membrane triggers the conversion of the light absorbing pigment diadinoxanthin to diatoxanthin, which dissipates absorbed light energy as heat instead of passing it on to the photosystems (Gustafsson et al. 2014). Alternately, state transitioning (qT) involves the reversible phosphorylation and resultant rearrangement of light harvesting pigments within PSII. This can decouple the light-harvesting pigments from the reaction centres and reduce the quantum yield of photosystem II (Falkowski and Raven 2007). In addition, the thylakoid membranes of Symbiodiniaceae photosystems I and II are within close physical proximity, which facilitates the bidirectional transfer of energy between these systems, a phenomenon which appears to increase during thermal stress (Slavov et al. 2016). Symbiodiniaceae have the ability to reorganise the positioning of photosystems I and II complexes along the thylakoid membrane, which 
is thought to be a photoprotective mechanism allowing a redirection of excess light energy away from the photosystems to avoid damage to the photosynthetic apparatus. This excess energy is converted to heat and quenched by chlorophyll P700+ which is an accessory pigment (Slavov et al. 2016). Lastly, photoinhibitory quenching (qI), occurs due to damage of the photosynthetic apparatus, which decreases the efficiency of the harvesting and conversion of light energy (Warner et al. 1999).

\subsubsection{The cellular pathways underlying photosynthetic dysfunction}

The initial stages of the current oxidative theory of coral bleaching are linked to the photosynthetic dysfunction of a coral's algal symbionts (Rehman et al. 2016). At high temperatures and irradiance, more energy is captured by the primary light-harvesting pigments and the photosynthetic electron transport chain than can be used for $\mathrm{CO}_{2}$ assimilation (Krause and Weis 1991, Szabó et al. 2005, Warner and Berry-Lowe 2006). This results in the overload of the photosynthetic machinery, causing photodamage and the production of ROS (Warner et al. 1999, Rehman et al. 2016, Warner and Suggett 2016). Various areas of vulnerability within the photosystem of Symbiodiniaceae have been identified:

1. Within the thylakoid membranes of the chloroplast, the D1 protein (part of the water-splitting complex in photosystem II), is particularly susceptible to destabilisation under stressful environmental conditions (Ohad et al. 1994, Warner et al. 1999, Robison and Warner 2006). Under ambient conditions, a repair complex keeps this machinery functional (Ohad et al. 1994). However, under thermal stress both the D1 protein and the repair complex become damaged (Warner et al. 1999, Takahashi et al. 2004, Hill et al. 2011). This can decrease the capacity of photosystem II to utilise absorbed light energy, causing a backup of excitation energy and photoinhibition (Warner et al. 1999). Notably, the capacity to repair thermally induced damage to the $\mathrm{D} 1$ protein is a potential area of functional diversity between Symbiodiniaceae species (Takahashi et al. 2004).

2. The Calvin cycle or the dark reactions of photosynthesis have been shown to reduce carbon fixation under elevated temperatures and light levels (Jones et al. 1998, Bhagooli 2013). In addition, chemical inhibition of the Calvin cycle has been observed to result in photoinhibition and bleaching in corals (Bhagooli, 2013). Evidence suggests that the site of Calvin cycle dysfunction is ribulose bisphosphate carboxylase oxygenase (Rubisco), which facilitates carboxylation (Lesser 1996). Symbiodiniaceae are unique amongst eukaryotes in their form of 
Rubisco (II), which is sensitive to thermally-induced alteration and has a low specifity for $\mathrm{CO}_{2}$ relative to $\mathrm{O}_{2}$ (Leggat et al. 1999, Lilley et al. 2010). The fixation of $\mathrm{O}_{2}$ instead of $\mathrm{CO}_{2}$ by Rubisco, or photorespiration, has no metabolic use, but may reduce excess $\mathrm{O}_{2}$, in turn reducing ROS production (Smith et al. 2005). Photorespiration induces the production of the Calvin cycle-inhibitor phosphoglycerate (Badger et al. 1998, Crawley et al. 2010). Thus, as photorespiration increases, less ATP and NADPH produced by the light reactions is utilised for carbon fixation, a process called 'sink limitation'. This is thought to lead to a backup of excitation energy in photosystem II, which can lead to dysfunction (Jones et al. 1998, Venn et al. 2008). However, it is worth noting that this energy sink limitation remains a hypothesis and has not been demonstrated in Symbiodiniaceae (Oakley et al. 2014b).

3. The thylakoid membrane can also be directly damaged by heat and light stress (Downs et al. 2013), causing changes in membrane lipid saturation (Tchernov et al. 2004). These changes result in decreased structural integrity of the thylakoid membrane, which disrupts electron transport across both photosystems I and II. Photosynthetic machinery continues to produce electrons but stops quenching them via ATP and NADPH production. These free electrons can then reduce photosynthetically derived $\mathrm{O}_{2}$, forming ROS (Tchernov et al., 2004). Notably, the threshold temperature at which membrane composition alters varies between Symbiodiniaceae genera and is diagnostic of their thermal sensitivity (Tchernov et al. 2004).

\subsubsection{Photo-repair}

If damage occurs to the photosystems as described above, Symbiodiniaceae have the capacity to repair this damage via de novo protein synthesis, and so restore functionality. For example, the D1 protein, which is susceptible to damage, can be repaired and replaced (Ohad et al. 1994).

Photoinhibtion can be repaired within hours of returning to non-stressful conditions (Gorbunov et al. 2001, Takahashi et al. 2009, Hill et al. 2011). In the context of coral bleaching, the capacity for photosystem repair is thought to be a determinant of thermal tolerance and bleaching susceptibility (Warner et al. 1999, Takahashi et al. 2004, 2008). This is exemplified by a study performed by Ragni et al. (2010), who investigated the relative investment of energy in photoprotection to avoid photodamage, or in the repair of the photosystems once photodamage had occurred in two strains of Symbiodiniaceae; A1, and the more thermally sensitive A1.1. When exposed to high light conditions, A1.1 exhibited greater photoinhibtion than A1. However, measurements of NPQ remained similar. In the presence of an inhibitor of D1 synthesis, photoinhibiton was similar, suggesting that, in this case, 
the more thermally-resistant taxon of alga invests more energy in photorepair mechanisms than photoprotective mechanisms. The activity of photosystem II is dependent on the net rates of photoprotection, photodamage and photorepair (Ragni et al. 2010), thus thermal tolerance is determined by the balance of all three of these processes.

\subsection{The effect of eutrophication on coral reefs}

In addition to the global scale threat of oceanic warming, coral reefs face stressors on a local scale as well. Anthropogenic presence along coastlines have led to declining water quality on coral reefs (Schaffelke et al. 2012). Municipal and industrial wastewater outputs, in conjunction with runoff from urbanised areas and agricultural land, has increased the levels of nitrogen and phosphorus in coastal waters (Howarth et al. 1996, Carpenter et al. 1998, Conley et al. 2009). Worldwide increased nutrient loading has been quantitatively linked to the degradation of coral reefs (Brown 1997, Fabricius 2005, DeVantier et al. 2006, Wooldridge 2009).

Increased nutrient levels, specifically elevated nitrogen, can cause enlarged symbiont populations (Muscatine et al. 1989a, Stambler et al. 1991, Marubini and Davies 1996). Increased symbiont densities can cause a shift in symbiotic balance, as they can become a carbon sink instead of a carbon source (Ezzat et al. 2015, Wooldridge 2016), which can have negative consequences for coral physiology. Corals in nutrient-enriched waters have been observed to exhibit reduced tissue thickness (McGuire and Szmant 1997, Cruz-Pinon et al. 2003), gamete production (Tomascik and Sander 1987, Loya et al. 2004), and skeletal growth rates (Marubini and Davies 1996, Ferrier-Pagès et al. 2001). In addition, enlarged symbiont populations have been linked to an enhanced thermal bleaching response (Wooldridge et al. 2017). One explanation for this observation is an increased total production of reactive oxygen species (Cunning and Baker 2013). Alternately, elevated Symbiodiniaceae densities also result in an increased demand for carbon and phosphate, which if not met induces physiological dysfunction (Wiedenmann et al. 2013, D’Angelo and Wiedenmann 2014, Rosset et al. 2017). Phosphate starvation of Symbiodiniaceae due to a severely imbalanced nitrogen to phosphorus ratio has been shown to induce a shift in the lipid composition of the thylakoid membrane from phospholipids to sulpholipids, which is thought to alter the ionic character of the membrane, reducing the threshold for thermal and light induced damage (Wiedenmann et al. 2013). This highlights the importance of the relative availability of nitrogen to phosphorus in determining the impact of eutrophication on coral reefs. It is essential to characterise the response of corals and their dinoflagellate symbionts to different levels of eutrophication to inform ecologically relevant nutrient input limits and facilitate the effective management of coral reefs (Brodie et al. 2017b). 


\subsection{Aims of this study}

The phenomenon of coral bleaching has been the subject of many research efforts, as any advance in knowledge is a step towards the conservation of the important coral reef ecosystem. Elevated sea surface temperature due to global warming is the most prominent trigger of coral bleaching (HoeghGuldberg et al. 2007, Hughes et al. 2017). Thus, characterising the physiological response of both the host and symbiont to varying degrees of thermal stress is central to this field of research. However, many knowledge gaps remain, particularly with regards to the cellular mechanisms underlying physiological responses to stress (Weis 2008). This lack of fundamental knowledge is a concern in the face of global coral reef decline.

While the stressors associated with global warming remain the hardest to counter due to their scale, throughout the literature the importance of local stressors in determining the resilience of coral reef ecosystems has been highlighted as a point of focus (Douglas 2003, Hughes et al. 2003). For example, water quality, specifically the input of nutrients such as nitrogen and phosphorus into coastal ecosystems can have a dramatic effect on Symbiodiniaceae physiology (Wiedenmann et al. 2013, Ezzat et al. 2015, Rosset et al. 2017). Of particular importance is how the ratio of nitrogen to phosphorus determines the thermal tolerance of Symbiodiniaceae and, in turn, the susceptibility of the holobiont to bleaching (Wiedenmann et al. 2013, Rosset et al. 2017). In order to facilitate the effective management of local stressors, it is critical that we gain a greater understanding of the interaction between these factors and global threats such as increasing sea surface temperatures (D'Angelo and Wiedenmann 2014), as this will ultimately determine the persistence of these valuable ecosystems into the future.

This thesis aims to characterise the physiological and cellular response of the symbiotic dinoflagellate Breviolum minutum (ITS2 'type' B1), to both thermal stress and nutrient availability. This Symbiodiniaceae species was chosen as it is considered moderately robust to thermal and oxidative stress (Wietheger et al., 2015), and thus occupies an intermediate position on the spectrum of speciesspecific thermal tolerance (Swain et al. 2017). Moreover, it is the native symbiont of the sea anemone Exaiptasia pallida (commonly known as 'Aiptasia'), the globally-adopted model system for the study of the cnidarian-dinoflagellate symbiosis (Weis et al. 2008). The thesis aim was addressed by the following objectives.

1) To compare the physiological response to a rapid versus slow temperature increase in two strains of B. minutum. 
The first objective was addressed by performing a range of physiological measurements on two $B$. minutum strains in culture (culture IDs: NZ01 and FlAp2), exposed to a temperature change from $25^{\circ} \mathrm{C}$ to $35^{\circ} \mathrm{C}$ either immediately or over one week. The physiological measurements performed were: symbiont population density change (beginning and end), chlorophyll fluorescence with Pulse-Amplitude-Modulation (PAM) fluorometry, photosynthetic and respiratory oxygen flux using a chamber and oxygen probe, and alkaline phosphatase activity (APA) and chlorophyll quantification with colorimetric and fluorometric assays, respectively. It was hypothesised that the two strains of B. minutum would have different physiological responses to temperature due to epigenetic or phenotypic differences between populations. It was also hypothesised that the algae exposed to a rapid increase in temperature would show more significant signs of physiological dysfunction than those exposed to a more gradual temperature, reflecting a heat shock versus acclimation response.

2) To determine the influence of nutrient supply on the proteomic response to elevated temperature of B. minutum (culture ID: FlAp2), and so characterise the detailed cellular responses of this symbiotic dinoflagellate.

The second objective was addressed by utilising powerful proteomics techniques (LC-ESI-MS/MS, Liquid chromatography-electrospray ionisation - tandem mass spectrometry), to characterise the proteome of algae under different nutrient regimes at control $\left(25^{\circ} \mathrm{C}\right)$ and elevated temperatures $\left(34^{\circ} \mathrm{C}\right)$. In addition, various physiological measurements were taken to corroborate trends of protein expression (the same measurements described above). It was hypothesised that protein abundance would alter in response to both temperature and nutrient regime, and that there would be an interaction between these two environmental factors on the Symbiodiniaceae proteome. This proteomic approach had yet to be applied to Symbiodiniaceae, thus facilitating novel insight into the cellular ecophysiology of the Symbiodiniaceae.

Chapters 2 and 3 are written as independent manuscripts. Thus, there may be some repetition in content between chapters (mainly introductory material). Lastly, Chapter 4 considers research question that arise from the results of this thesis, and how these findings are significant in the context of knowledge-based reef management. 


\section{Chapter 2: Characterisation of the physiological response of two Breviolum minutum strains to a slow versus rapid temperature increase}

\subsection{Introduction}

\subsubsection{Cnidarian-dinoflagellate symbiosis and the success of coral reefs}

Coral reefs are one of the most productive and diverse places on Earth and support approximately a third of the world's fish species (Reaka-Kudla et al. 1997, Reaka-Kudla 2001). This ecosystem is underpinned by the symbiosis between dinoflagellate algae from the family Symbiodiniaceae and cnidarian stony corals. These symbiotic algae reside within the gastrovascular cavity of their cnidarian host, providing the coral with photosynthates that support up to $100 \%$ of its metabolic requirements (Muscatine et al. 1981, Grottoli et al. 2006, Tremblay et al. 2012), facilitating coral growth and reproduction (Muscatine et al. 1984, Davies 1991). In return, the dinoflagellate algae receive protection, a stable place in the water column and coral waste products such as ammonium (Venn et al. 2008, Yellowlees et al. 2008). The transfer of nitrogen from the host to the symbiont facilitates nitrogen recycling and retention and is thought to underlie the success of coral reefs in the oligotrophic tropical waters (Muscatine and Porter 1977, Wang and Douglas 1998, Wang and Douglas 1999).

\subsubsection{Cnidarian bleaching and thermal stress}

Healthy corals contain millions of symbionts per square centimetre of tissue. However, when an environmental perturbation occurs, symbionts themselves can die due to stress and/or they are expelled from coral tissue, causing the coral to lose its pigmentation (see Figure 1.3) (Douglas 2003, Weis 2008). This phenomenon is called 'coral bleaching'. Bleached corals suffer nutrient deprivation, increased susceptibility to disease, and mortality if a symbiosis cannot be re-established (Goreau and Macfarlane 1990, McClanahan 2004). The most prominent causes of bleaching are elevated temperatures and irradiation. It is widely accepted that the global increase in coral bleaching over the last century is a consequence of elevated sea surface temperatures due to global warming (Hughes et al. 2003, Hughes et al. 2017). 
Thermal stress causes physiological dysfunction in both the coral host and its endosymbionts, leading to the formation of reactive species, of which there are two primary forms: reactive oxygen (ROS) and nitrogen species (RNS). On the level of the symbiont, elevated temperatures and irradiance can cause the dysfunction of Symbiodiniaceae's photosynthetic apparatus, through overexcitation, photoinhibition and sink limitation (Jones et al. 1998, Warner et al. 1999, Tchernov et al. 2004, Hill et al. 2011) (see section 1.3.3 of the general introduction for details). These processes lead to the formation of ROS (Lesser 2006, Warner and Suggett 2016), which may subsequently leak into host tissue (Saragosti et al. 2010, Rehman et al. 2016). Additionally, thermally-induced damage to the host mitochondrion and endoplasmic reticulum can generate high levels of ROS (Dunn et al. 2012, Lutz et al. 2015, Oakley et al. 2017, Ruiz-Jones and Palumbi 2017). In concert, there is evidence of NO production in response to thermal stress by both the cnidarian host (Perez and Weis 2006, Hawkins and Davy 2013, Hawkins et al. 2014), and its symbiotic dinoflagellates (Trapido-Rosenthal et al. 2001, Bouchard and Yamasaki 2008, Hawkins and Davy 2012, 2013). These reactive species are thought to elicit an immune response in the host (Weis 2008, Davy et al. 2012), which acts to remove symbionts from host tissue by several mechanisms, such as exocytosis, symbiont digestion, and most prominently host cell apoptosis, or programmed cell death (Weis 2008, Tchernov et al. 2011).

\subsubsection{Symbiont diversity and the need to better understand intra-species variability}

Coral bleaching susceptibility is determined by the thermal tolerance of both the coral itself and the symbiont type(s) it contains (Berkelmans and Van Oppen 2006, Baird et al. 2009, Quigley et al. 2018). Within the Symbiodiniaceae family, corals associate with four phylogenetic groups or genera: Symbiodinium (Clade A), Breviolum (Clade B), Cladocopium (Clade C), and Durusdinium (Clade D) (Carlos et al. 1999, LaJeunesse 2001, Pochon and Gates 2010, Pochon et al. 2014, LaJeunesse et al. 2018). There is also further diversity within these genera, previously known as subcladal phylotypes, and now defined as species, which is identified through sequencing the second internal transcribed spacer (ITS2) region of the nuclear ribosomal RNA gene (Silverstein et al. 2011, Franklin et al. 2012, Thornhill et al. 2014). This genetic diversity is reflected in physiological differences between both Symbiodiniaceae genera and species, which affects how 'valuable' they are to their cnidarian host as a symbiotic partner. For example, species may differ in their ability to produce photosynthates (Cantin et al. 2009, Starzak et al. 2014), take up nutrients (Baker et al. 2013), and tolerate thermal stress (LaJeunesse et al. 2009, Swain et al. 2017). With regards to thermal stress, differential tolerance between types has been connected to thylakoid membrane composition (Tchernov et al. 2004), ability 
to repair photosystem damage (Takahashi et al. 2009, Ragni et al. 2010), and combat oxidative stress (McGinty et al. 2012, Krueger et al. 2014). These physiological differences are significant on an ecosystem level, as the algal type(s) that reside within a coral colony contribute to both coral productivity and bleaching resistance (Coffroth 2005, Berkelmans and Van Oppen 2006, Hume et al. 2015). Although, many corals only associate with particular symbiont species (Fabina et al. 2012), there is evidence that the Symbiodiniaceae species in a coral may change in response to elevated light and/or temperature (Baker 2001, Reich et al. 2017), providing a possible mechanism of adaption to changing environmental conditions (Buddemeier and Fautin 1993). Therefore, understanding the different physiological capabilities of the Symbiodiniaceae family across its diversity will inform both the prediction and remediation of coral bleaching events.

Despite a growing body of literature that has ranked the thermal tolerance of different Symbiodiniaceae genera, only a fraction of the possible phylotype comparisons have been performed (Swain et al. 2017). Furthermore, inconsistencies between studies suggest that there may be differential thermal tolerance within genera and even within species (Swain et al. 2017). Although the ITS2 marker is the established method for species-level identification of the Symbiodiniaceae family, the diversity identified by this marker may not reflect thermal tolerance, as different populations may display phenotypic plasticity (Howells et al. 2012, Swain et al. 2017). This concept is supported by a foundational study by Howells et al., (2012), which was the first study to provide evidence for differential thermal tolerance between populations of the same species of Symbiodiniaceae. The thermal tolerance of two populations of the generalist Symbiodiniaceae type C1, extracted from the coral Acropora millepora from either South Molle Island (average maximum temperature 28.2 ${ }^{\circ} \mathrm{C}$ ), or Magnetic Island (average maximum temperature $32{ }^{\circ} \mathrm{C}$ ), Australia, were compared when in symbiosis with A. millepora from a third Australian reef location (Miall Island). When subjected to heat stress at $32{ }^{\circ} \mathrm{C}$, symbionts from Magnetic Island displayed a higher photochemical performance and survival than those symbionts from Molle Island, both in culture and symbiosis. Furthermore, juvenile corals associated with symbionts from Magnetic Island exhibited accelerated growth when exposed to a temperature of $32^{\circ} \mathrm{C}$, while those corals housing Molle Island symbionts responded to this increase in temperature with severe bleaching. This demonstrates that coral thermal tolerance can be shaped by the adaption of its photosymbionts to local environmental factors, emphasising the importance of further studies which characterise the thermal sensitivity of Symbiodiniaceae species on a population level. 


\subsubsection{The capacity for Symbiodiniaceae to adapt/ acclimate to climate change}

The ecophysiology of Symbiodiniaceae directly affects the thermal tolerance of the cnidarian-algal holobiont, and thus the capacity of Symbiodiniaceae to adapt to global warming is fundamental to the persistence of coral reefs (Takahashi et al. 2013). The potential for genetic adaption of Symbiodiniaceae populations is high due to their haploid genomes (Santos and Coffroth 2003), fast generation times (Wilkerson et al. 1988), large population sizes (Drew 1972, Littman et al. 2008) and genetic isolation between populations (Santos et al. 2003, Howells et al. 2009). The possibility of genetic adaption is illustrated by Symbiodinium thermophilum, which originated from a thermally tolerant sub-population of an ancestral taxonomic group and adapted to a rapidly changing climate in the Holocene (Hume et al. 2015). Today this species facilitates corals survival in the world's hottest seas (Persian/Arabian Gulf) (Hume et al., 2015). However, it remains uncertain whether corals and their symbionts will be able to genetically adapt fast enough to global warming, due to the current rate of temperature change (Donner et al. 2005, Pandolfi 2015).

Alternately, the persistence of coral reefs may be determined by phenotypic changes that are independent of genotype, classified as acclimatisation/acclimation (Prosser 1991, Edmunds and Gates 2008). Plants have the capacity to acclimate to stressful environmental conditions (Bruce et al. 2007). The molecular basis of these physiological changes reflects either the accumulation of signalling proteins or transcription factors, which activate molecular defence mechanisms against this stressor, or epigenetic change, whereby the genetic code itself remains unchanged but the accessibility of the genetic code changes, altering gene expression (Hochachka 2002, Madlung and Comai 2004, Bruce et al. 2007). Symbiodiniaceae have been observed to alter gene expression in response to their environment (Davies et al. 2018), and when under experimentally induced thermal stress (Gierz et al. 2017). These epigenetic changes may underlie observed thermal acclimation of Symbiodiniaceae in both the field and laboratory. Thermal history is integral to this acclimation process, as sub-lethal exposure to a stressor is required to promote phenotypic change (Bruce et al. 2007). For example, meta-analyses of field data have revealed that corals historically exposed to higher temperature variability are more resistant to thermally induced bleaching than counterparts that reside in parts of the reef where temperatures are more stable (Carilli et al. 2012, Ainsworth et al. 2016, Safaie et al. 2018). The importance of the rate of temperature change in inducing thermal acclimation has also been experimentally tested, demonstrating that a period of moderate thermal stress which precedes a more severe period can reduce symbiont loss (Middlebrook et al. 2008, Ainsworth et al. 2016), and different heating rates alter Symbiodiniaceae physiology (in hospite) at a given temperature (Middlebrook et al. 2010). In addition, increasing the growth temperatures of Symbiodiniaceae 
cultures has been found to increase thermal tolerance (Díaz-Almeyda et al. 2011, Takahashi et al. 2013, Chakravarti et al. 2017). Further characterisation of the capacity of Symbiodiniaceae to acclimate to elevated temperatures and the time frame in which this process takes place, is integral to our understanding of the response of the cnidarian-algal symbiosis to global warming.

\subsubsection{Insight provided by this study}

As global warming increases sea surface temperatures, corals are approaching or have surpassed their maximum thermal limits (Fabricius et al. 2007, Hoegh-Guldberg et al. 2007). The survival of these animals which form the basis of tropical reef ecosystems will be determined by their ability to adjust to these rapidly changing conditions (Coles and Brown 2003). Both partners of the symbiosis must survive these warming conditions; thus, both must adapt or acclimate. Thermally tolerant hostsymbiont genotype combinations could be the determinant of the survival of corals (Baskett et al. 2009, LaJeunesse et al. 2010, Parkinson and Baums 2014). To this end, the genetic and physiological diversity of Symbiodiniaceae provides a sound basis for adaption/ acclimation. However, due to this diversity, despite the extensive studies analysing the thermal tolerance of Symbiodiniaceae (Swain et al., 2017 and references therein), few studies have compared the thermal tolerance of two strains of the same species (Bayliss et al. , Howells et al. 2012), which may provide insight into physiological plasticity on a population level (Parkinson et al. 2016).

This study addressed this knowledge gap, aiming to compare the physiological response to a rapid versus slow temperature increase in two strains of Breviolum minutum. This aim was addressed by performing a range of physiological measurements on two B. minutum strains in culture (NZ01 and FlAp2), exposed to a temperature change from $25^{\circ} \mathrm{C}$ to $35^{\circ} \mathrm{C}$ either immediately or over one week. The physiological measurements performed were: symbiont population density change (beginning and end), Pulse-Amplitude-Modulation (PAM) fluorometry, chlorophyll quantification, oxygen flux using a chamber and oxygen probe, and alkaline phosphatase activity with an assay (APA). It was hypothesised that the two strains of B. minutum would have different physiological responses to temperature due to epigenetic or phenotypic differences between populations (Bayliss et al. , Howells et al. 2012, Parkinson et al. 2016). It was also hypothesised that a slow versus rapid increase in temperature would cause less thermally induced physiological dysfunction, reflecting thermal acclimation versus shock. This is based on the idea that the slower temperature increase will allow time for epigenetic change and the de novo synthesis of proteins (Prosser 1991, Hochachka 2002, Madlung and Comai 2004, Bruce et al. 2007, Edmunds and Gates 2008, Takahashi et al. 2013), while 
the dramatic increase in temperature will not. With regards to this, these two temperature treatments would provide greater insight into differential physiological plasticity between the two algal strains.

\subsection{Materials and methods}

\subsubsection{Experimental design}

Bleaching thresholds depend on geographic location but generally range between 27 to $36.8^{\circ} \mathrm{C}$ (Hoegh-Guldberg 1999, Fitt and Cook 2001, Coles and Brown 2003, Hoegh-Guldberg et al. 2004, Jokiel 2004). Thus, an upper-temperature maximum of $35^{\circ} \mathrm{C}$ was chosen for this experiment, to ensure that a physiological response was observed while remaining ecologically-relevant. The effect of heating rate is explored by comparing a dramatic temperature increase from 25 to $35^{\circ} \mathrm{C}$ over two different time scales (see Figure 2.1). For one temperature treatment, algal cultures were moved immediately from 25 to $35^{\circ} \mathrm{C}$, and maintained at $35^{\circ} \mathrm{C}$ for $72 \mathrm{~h}$. While extreme, similar temperature fluctuations have been observed on coral reefs in the Persian Gulf, where temperatures can vary up to $9^{\circ} \mathrm{C}$ per day (Coles 1997, Coles and Brown 2003, Hume et al. 2013). Alternately, for the second temperature treatment the temperature of the cultures was increased from $25^{\circ} \mathrm{C}$ to $35^{\circ} \mathrm{C}$ over a seven day period (an increase of $1.6^{\circ} \mathrm{C}$ on day 1 , and $1.2^{\circ} \mathrm{C}$ every day thereafter), similar to heating rates used in previous studies (Howells et al. 2012, McGinley et al. 2012b, Fujise et al. 2014), and then also maintained at $35^{\circ} \mathrm{C}$ for $72 \mathrm{~h}$. Notably, for both temperature treatments the FlAp2 cultures were maintained at $35^{\circ} \mathrm{C}$ for $48 \mathrm{~h}$ instead of $72 \mathrm{~h}$ as a dramatic drop in the maximum quantum yield to 0.2 (see Figures $2.4 \& 2.5$ below), prompted an earlier cut off of the experimental period as the cultures were in danger of dying rendering them useless for further physiological measurements.

Thus, the experimental period was 24 days long in the case of NZ01 and 23 days long in the case of FLAp2. This includes two weeks of acclimation before temperatures were raised for treatments cultures; one week kept in a glass conical flask in the culture cabinet at $25^{\circ} \mathrm{C}(12: 12$ light, dark cycle, $\mathrm{PAR}=40 \mu \mathrm{mol}$ photons $\mathrm{m}^{-2} \mathrm{~s}^{-1}$, warm fluorescent lamps), in Red Sea pro-salt artificial seawater (low nutrient conditions), as opposed to the standard f/2 medium, and one week kept in $15 \mathrm{ml}$ Falcon tubes, in temperature-controlled water baths (12:12 light dark cycle, $\mathrm{PAR}=60-65 \mu \mathrm{mol}$ photons $\mathrm{m}^{-2} \mathrm{~s}^{-1}$, warm T12 fluorescent bulbs). Note that this experimental period was staggered by one day for each of the temperature treatments and the controls as the respirometry measurements could not have been completed in one day for all the experimental cultures. For the control and each temperature treatment 
there were 14 biological replicates for NZ01 and 16 for FlAp2 due to the greater availability of this culture (see Figure 2.2).

\section{Temperature controlled water baths}

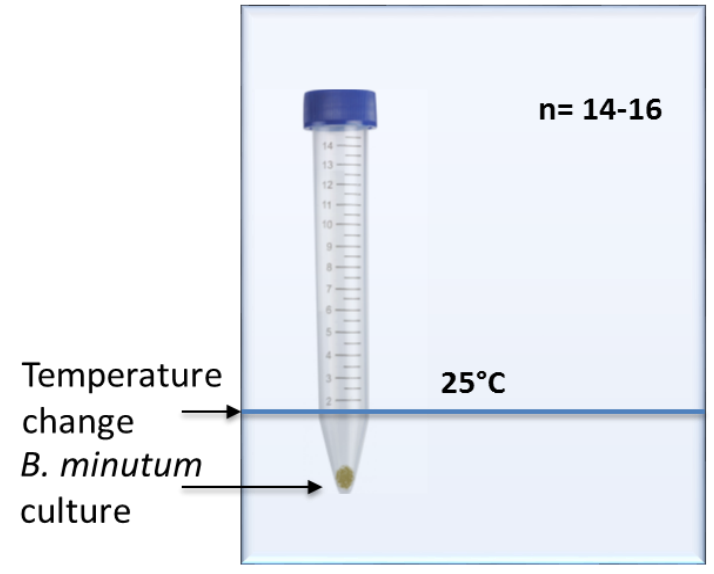

Control

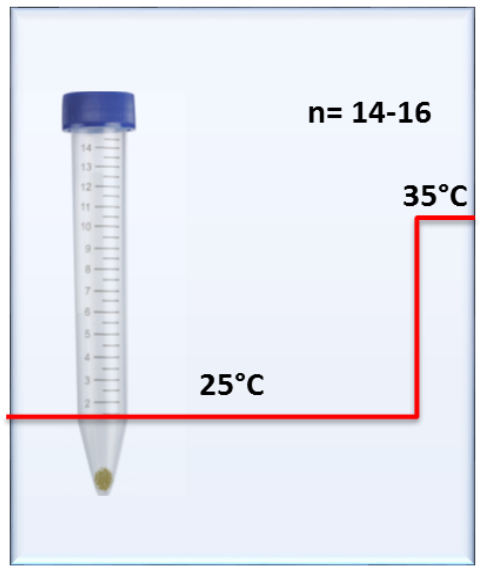

Rapid temperature increase treatment

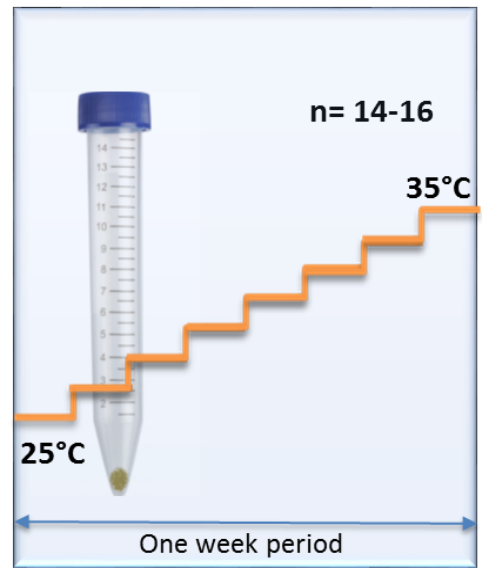

Slow temperature increase treatment

Figure 2.1: Diagram depicting the different temperature treatments

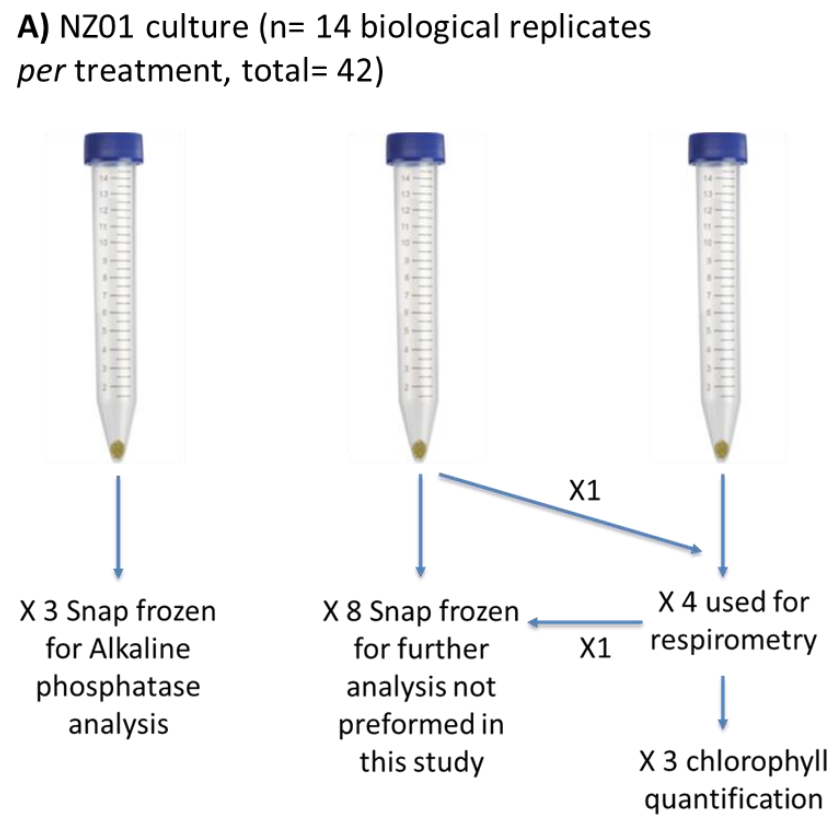

X14 used for chlorophyll fluorescence and population density measurements
B) FIAp2 culture ( $n=16$ biological replicates per treatment, total $=48$
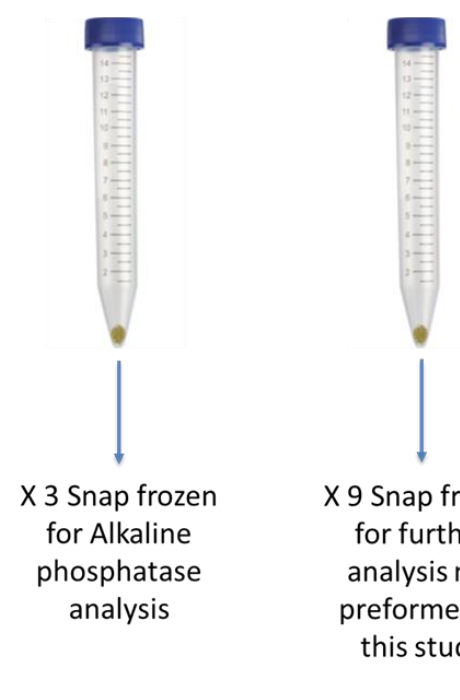
X 9 Snap frozen for further analysis not preformed in this study

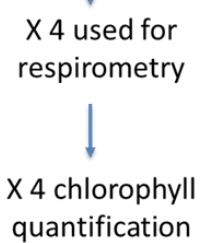

X16 used for chlorophyll fluorescence and population density measurements

Figure 2.2: Diagram depicting the allocation of biological replicates towards the various physiological measurements preformed in this study. 


\subsubsection{Experimental organisms}

The Symbiodiniaceae species used here was Breviolum minutum (ITS2 'type' B1), the homologous symbiont of the model cnidarian Aiptasia. Breviolum minutum is considered moderately robust to thermal and oxidative stress (Wietheger et al. 2015) and thus occupies an intermediate position on the spectrum of species-specific thermal tolerance (Swain et al. 2017).

Both the NZ01 and FLAp2 culture strains were genetically identified as B. minutum, as confirmed by sequencing of the ITS2 region (visuals of cultures are provided in Figure 2.3 below). The NZ01 strain of Symbiodiniaceae was isolated from the Davy lab clonal Aiptasia stock using sequential inoculations in $\mathrm{f} / 2$ medium and germanium oxide to prevent the growth of diatoms at the beginning of 2017. Alternately, the FlAp2 culture was isolated from an Aiptasia stock sourced from the Florida Keys by Scott Santos (Auburn University) and received by the Davy lab in 2012. Since isolated or received by the Davy group, both cultures have been maintained in $\mathrm{f} / 2$ medium, at $25^{\circ} \mathrm{C}$, on a $12: 12$ light dark cycle in a temperature-controlled incubator, $\mathrm{PAR}=40 \mu \mathrm{mol}$ photons $\mathrm{m}^{-2} \mathrm{~s}^{-1}$ (warm fluorescent lamps).

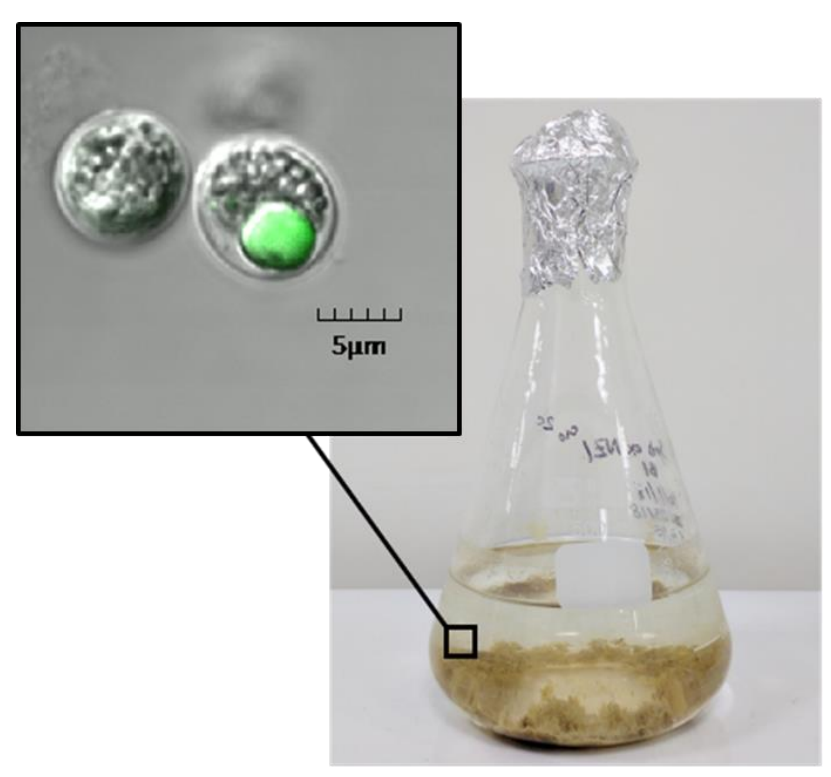

NZ01 Strain

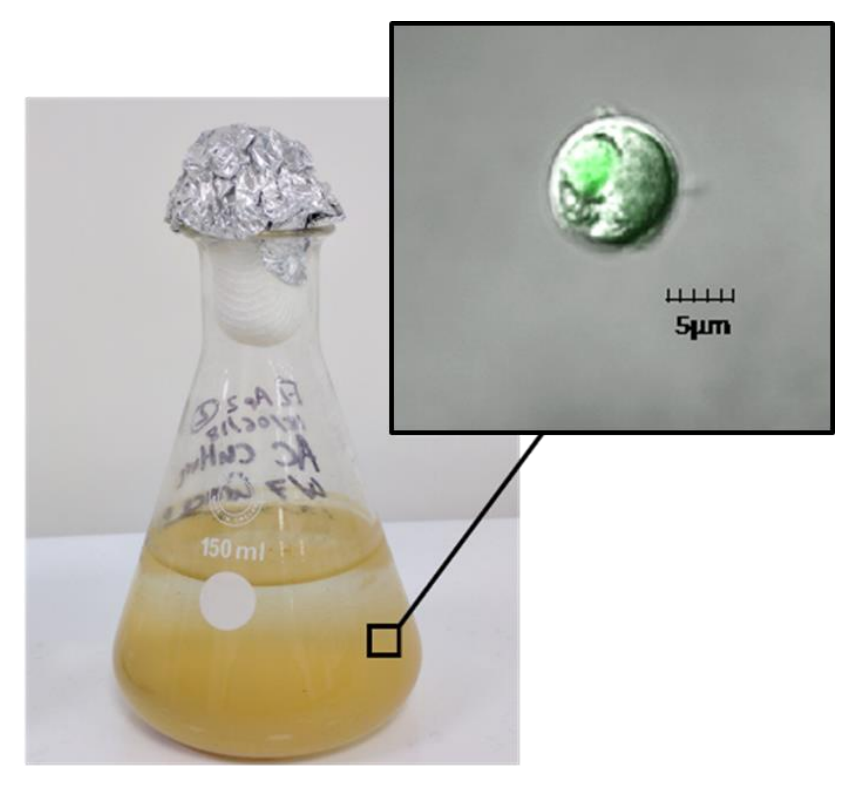

FIAp2 Strain

Figure 2.3: NZ01 and FlAp2 strains of Breviolum minutum. 


\subsubsection{Experimental procedure}

\section{Setting up the cultures for the experiment}

The B. minutum cultures are normally maintained in $\mathrm{f} / 2$ medium, at $25^{\circ} \mathrm{C}$, on a $12: 12$ light dark cycle in a temperature-controlled incubator, $\mathrm{PAR}=40 \mu \mathrm{mol}$ photons $\mathrm{m}^{-2} \mathrm{~s}^{-1}$ (warm fluorescent bulbs). At the beginning of the experimental period an unopened glass pipette and a pipette pump was used to extract $20 \mathrm{ml}$ of $B$. minutum culture (either NZ01 or FLAp2) from the source flask and transferred into a new $50 \mathrm{ml}$ falcon tube. This tube was then centrifuged at $20,000 \times \mathrm{g}$ for 6 minutes. The supernatant was removed leaving the algal pellet. This pellet was then re-suspended in $10 \mathrm{ml}$ of artificial sea water (ASW, Red sea coral pro-salt) and vortexed. This process was repeated twice more to wash the algal cells of the $\mathrm{f} / 2$ medium. The $B$. minutum pellet was then resuspended in pro-salt mix and transferred into a new autoclaved glass conical flask. After a one-week acclimation to this altered nutrient medium, an unopened glass pipette was used to transfer the culture from the conical flask into a new $50 \mathrm{ml}$ tube. Cell counts were performed using a haemocytometer to determine the density of $B$. minutum in the $50 \mathrm{ml}$ tube. Based on the density calculation the B. minutum culture was aliquoted into $15 \mathrm{ml}$ falcon tubes so that approximately 3 million cells were in each tube ( $\mathrm{n}=42$ for NZ01, $\mathrm{n}=48$ for FLAp2). A sub-sample was taken from each tube for initial density cell counts, and the total volume of the medium in each tube was increased to $8 \mathrm{ml}$. These $15 \mathrm{ml}$ tubes were then transferred into their destined experimental tank. $4 \mathrm{ml}$ of the pro-salt medium was exchanged daily (half of the total volume), to ensure a relatively consistent nutrient regime.

\section{Photo-physiological measurements}

The diving-PAM chlorophyll fluorometer (Walz, Effeltrich, Germany), was used to measure chlorophyll fluorescence daily at $11 \mathrm{am}$ (diel cycle $5 \mathrm{am}-5 \mathrm{pm}$ ). The instantaneous fluorescence yield $\left(F_{t}\right)$ and the maximum fluorescence yield $\left(F_{m}\right.$ ') were measured in actinic light. Then after 20 minutes dark adaption the minimal fluorescence yield $\left(\mathrm{F}_{0}\right)$ and the dark-adapted maximum fluorescence yield $\mathrm{F}_{\mathrm{m}}$ were also measured. These measurements were then used to calculate the following parameters;

Effective PS11 quantum yield $(\phi \mathrm{PS})=\Delta \mathrm{F} / \mathrm{F}_{\mathrm{m}}{ }^{\prime}=\left(\mathrm{F}_{\mathrm{m}}{ }^{-}-\mathrm{F}_{\mathrm{t}}\right) / \mathrm{F}_{\mathrm{m}}$ ' $($ Genty et al. 1989)

Maximum quantum yield (photosynthetic efficiency) $=\mathrm{F}_{\mathrm{v}} / \mathrm{F}_{\mathrm{m}}=\left(\mathrm{F}_{\mathrm{m}}-\mathrm{F}_{0}\right) / \mathrm{F}_{\mathrm{m}}$ (Kitajima and Butler 1975) 


\section{Sample processing at the end of the experimental period}

At the end of the experimental period all biological replicates were removed from their water bath and centrifuged for 8 minutes at $2000 \times \mathrm{g}$. The pro-salt culture medium was then removed from each tube, leaving an algal pellet. The algal pellet was then re-suspended in $500 \mu \mathrm{l}$ of ASW medium and transferred into a $2 \mathrm{ml}$ tube. A further $700 \mu \mathrm{l}$ of ASW medium was added to the $15 \mathrm{ml}$ tube to collect any algae left behind. This was then also transferred to the $2 \mathrm{ml}$ tube making the total volume $1.2 \mathrm{ml}$. $200 \mu \mathrm{l}$ was then extracted from the $2 \mathrm{ml}$ tube for cell counts. Four of these $2 \mathrm{ml}$ tubes were then set aside for respirometry measurements, followed by chlorophyll quantification. The remaining tubes were spun down for 3 minutes at $2000 \times \mathrm{g}$ and the supernatant was removed. These $2 \mathrm{ml}$ tubes containing algal pellets were then snap frozen in the $-80{ }^{\circ} \mathrm{C}$ freezer (see Figure 2.2 for allocation of biological replicates).

\section{Population growth}

Due to the high volume of cell counts needed for this study, I developed and refined a new protocol for Symbiodiniaceae cell counts using the high throughput confocal IN Cell analyser $6500 \mathrm{HS}$ microscope, followed by image analysis with IN Carta image analysis software.

$100 \mu \mathrm{l}$ aliquots were taken from the culture extracts that were saved for cell counts at the beginning and end of the experimental period and transferred into a $2 \mathrm{ml}$ tube. $900 \mu \mathrm{l}$ of sodium chloride solution with $30 \mathrm{mM}$ of EDTA was then added to the $2 \mathrm{ml}$ tube. The cells were then vortexed thoroughly, followed by being drawn up with a $2.5 \mathrm{ml}$ syringe and blasted out back into the tube to eliminate clumps. A further $1000 \mu \mathrm{l}$ of sodium chloride solution was then added to the tube, making the total volume $2000 \mu \mathrm{l} .12$ replicates of $10 \mu \mathrm{l}$ aliquots were taken from this solution and loaded into a 396 well plate, vortexing between each replicate.

Images of each well were taken at x10 magnification using the high throughput confocal IN Cell analyser $6500 \mathrm{HS}$ microscope, with the far-red laser (642 nm, 4\%, exposure: 0.45, aperture 1.12), which promotes the auto-fluorescence of Symbiodiniaceae chlorophyll. These images were then loaded into IN Carta image analysis software and the number of cells in each well were counted using the 'count nuclei' function. The count produced by the software was then multiplied to account for dilution and used to estimate the number of cells in the culture of interest. Using the cell counts at the beginning and end of the experimental period, the percent population increase was calculated. 


\section{Measurements of photosynthetic and respiratory oxygen flux}

For each B. minutum strain and treatment (controls and two temperature treatments), four biological replicates were transferred into a $1.76 \mathrm{ml}$ respirometry chamber containing a stir bar. Oxygen flux was measured using the Presens Fibox 3 minisensor oxygen meter and the Oxyview-PST3 software (version 6.02). To determine the respiration rate oxygen levels were first measured for 30 minutes in the dark. Oxygen evolution was then measured in the light for 30 minutes (PAR $=80 \mu \mathrm{mol}$ photons $\mathrm{m}^{-}$ $\left.{ }^{2} \mathrm{~S}^{-1}\right)$. These measurements were performed in a water bath to sustain experimental temperatures, with the treatment cultures maintained at $35^{\circ} \mathrm{C}$ and the controls maintained at $25^{\circ} \mathrm{C}$. From these measurements the respiration rate, net photosynthetic rate, gross photosynthetic rate, and the photosynthesis to respiration ratio was calculated. This value was then standardised per million cells.

\section{Chlorophyll quantification}

After respirometry was performed the B. minutum cultures were returned to their $2 \mathrm{ml}$ tube and spun down using the centrifuge for 3 minutes at $2000 \times \mathrm{g}$, after which the supernatant was removed. The remaining algal pellet was re-suspended in $70 \%$ ethanol, vortexed, and then kept in the $-20^{\circ} \mathrm{C}$ freezer in a dark box (chlorophyll can break down in light). $70 \%$ ethanol was used so the cells did not become dissociated as these same cells could later be imaged with the confocal microscope. These tubes were spun down and $300 \mu \mathrm{l}$ of supernatant was removed and put in a new $1.7 \mathrm{ml}$ tube. $1.5 \mathrm{ml}$ of $100 \%$ ethanol was then added to $300 \mu$ of the supernatant to reach a final concentration of $95 \%$ ethanol. This supernatant was transferred into a 96 well plate and the absorbance was read with a spectrophotometer at the following wave lengths; 664.1, 648.6 and $470 \mathrm{~nm}$. The absorbance of the blank (95\% ethanol) was then subtracted from the absorbance values. These absorbance values were substituted into the following equations to calculate chlorophyll a and carotenoid concentrations (Lichtenthaler and Buschmann 2001), then standardised per million cells:

\section{Chlorophyll a concentration:}

$C_{a}(\mu \mathrm{g} / \mathrm{ml})=13.36 A_{664.1}-5.19 A_{648.6}$

The concentration of the sum of carotenoids:

$C_{(x+c)}(\mu \mathrm{g} / \mathrm{ml})=\left(1000 A_{470}-2.13_{C a}\right) / 209$ 


\section{Nutritional response to thermal stress}

The nutrient condition of Symbiodiniaceae has been found to alter thermal tolerance (Wiedenmann et al. 2013, Ezzat et al. 2016a, Rosset et al. 2017), possibly due to modified thylakoid membrane composition (Wiedenmann et al. 2013), which has also been found to be diagnostic of differential thermal sensitivity between Symbiodiniaceae types (Tchernov et al. 2004). It was the intention to investigate the accumulation of both starch and lipid bodies under different temperature treatments using confocal microscopy. These storage products can provide energy and a source of carbon (Vitova et al. 2015). It was predicted they might be utilised under thermal stress due to a reduction in carbon fixation (Jones et al. 1998). The analysis of starch and lipid bodies was unable to be optimised in the time frame of this study due to equipment failure (see appendix section A1.2 for progress that was made). However, an assay of alkaline phosphatase activity (APA), which is involved in the recycling of inorganic phosphorus, was optimised (see below).

\section{Alkaline Phosphatase assay procedure}

Alkaline phosphatase converts organic phosphorus into inorganic phosphorus $\left(\mathrm{P}_{\mathrm{i}}\right)($ Feder 1973). This allows a regeneration of $\mathrm{P}_{\mathrm{i}}$ in phosphate deficient environments. Alkaline phosphatase activity (APA) has been found to increase in response to a decreased availability of $\mathrm{P}_{\mathrm{i}}$ in many marine autotrophs (Short et al. 1985, Lapointe 1987), including Symbiodiniaceae (Annis and Cook 2002, Wiedenmann et al. 2013). Phosphorus is essential to the thermal stress response in Symbiodiniaceae, with increased abundance increasing thermal tolerance (Wiedenmann et al. 2013, Ezzat et al. 2016a, Rosset et al. 2017). Thus, I hypothesised that APA will increase in response to elevated temperature. Previous studies analysing APA in Symbiodiniaceae have used a para-nitrophenyl phosphate (p-NPP) colorimetric assay (Annis and Cook, 2002; Wiedenmann et al. 2013). However, this assay was not efficiently sensitive, so instead a modified methylum belliferyl fluorometric assay was used (modified from Marx et al., 2001). This assay hasn't been used for dinoflagellates before, but the affinity of phosphatases for 4-methylumbelliferyl phosphate has been found to up to two orders of magnitude greater than p-NPP (Marx et al. 2001).

For each Breviolum minutum strain and treatment (controls and two temperature treatments), the alkaline phosphatase activity of four biological replicates was measured. At the end of the experimental period samples were snap frozen in $2 \mathrm{ml}$ tubes and stored in the negative $80{ }^{\circ} \mathrm{C}$ freezer. These samples were defrosted and washed three times in $50 \mu \mathrm{M}$ Tris-HCl buffer containing $1 \mu \mathrm{M}$ 
$\mathrm{MgCl}_{2}$ ( $\mathrm{pH} 10.3$ ) to remove any phosphate from remnant pro-salt mix. $0.5 \mathrm{ml}$ Tris- $\mathrm{HCl}$ buffer was added to the algal pellet, and the mixture was sonicated on ice at $15 \%$ output, for 10 pulses with a two second interval to liberate phosphatases from within the cell. A further $1 \mathrm{ml}$ of Tris- $\mathrm{HCl}$ buffer was added to the tube, along with $0.25 \mathrm{ml}$ of $100 \mu \mathrm{M}$ 4-methylumbelliferyl phosphate. This mixture was left to incubate in darkness at room temperature for exactly one hour. The reaction was terminated by the addition of $0.225 \mathrm{ml}$ of $1 \mathrm{M} \mathrm{NaOH}$, and the tubes were centrifuged for 3 minutes at $3200 \times \mathrm{g}$. Standards of 4-Methylumbelliferone were prepared and incubated in conjunction with the samples. $100 \mu \mathrm{M}$ stock solution was pipetted into $2 \mathrm{ml}$ centrifuge tubes and made up to $1.75 \mathrm{ml}$ with Tris- $\mathrm{HCl}$ buffer to make the following concentrations; $10.5 \mu \mathrm{M}, 5.5 \mu \mathrm{M}, 0.5 \mu \mathrm{M}, 0.25 \mu \mathrm{M}$ and $0.1 \mu \mathrm{M}$. 0.225 $\mathrm{ml}$ of $1 \mu \mathrm{M} \mathrm{NaOH}$ was also added to the standards after a one hour incubation, and the tubes were centrifuged for 3 minutes at $3200 \times \mathrm{g}$. A blank solution was made from $1.75 \mathrm{ml}$ of Tris- $\mathrm{HCl}$ buffer and $0.225 \mathrm{mls}$ of $1 \mu \mathrm{M} \mathrm{NaOH}$. $0.25 \mathrm{ml}$ of the blank solution, the various standard solutions and sample solutions were pipetted into a black 96 well plate, with five technical replicates for each solution. Fluorescence was measured using a Perkin Elmer Enspire 2300 multi-label microplate reader with an excitation wavelength of $365 \mathrm{~nm}$ and emission wavelength of $450 \mathrm{~nm}$. The blank absorbance was subtracted from the standard and sample absorbance values. The absorbance values of the MUB standards $(0.1 \mu \mathrm{M}-10.5 \mu \mathrm{M})$ were then used to create a standard curve. The equation of this standard curve was then used to calculate the concentration of MUB produced by alkaline phosphatase activity per hour, using the sample fluorescence values (y), and rearranging for $\mathrm{x}$ (concentration of MUB). This value was then standardised per million cells.

\subsubsection{Statistical analysis}

All statistical tests were performed with GraphPad Prism (7.04). Unpaired two tailed t-tests, not assuming constant variance, were used to compare quantum yield measurements of control and treatment measurements on a daily basis $(\alpha=0.01)$. In some cases the unpaired t-test identified significant differences between the quantum yield measurements of control and treatment cultures during the period where they were both kept at the same temperature. These discrepancies are likely due to biological variability between replicates and are not discussed in the results section as they do not contribute to the overall trends. For the effective quantum yield measurements of NZ01 cultures a repeated measures ANOVA was performed in place of an unpaired t-test, due to a consistent difference between control and treatment cultures throughout the experimental period. To determine how the control and treatment measurements changed over time each measurement from day 15 
onwards was compared with the average fluorescent measurement over the first six days of the active experiment when all cultures including treatments were kept at $25^{\circ} \mathrm{C}$ (control conditions) $(\alpha=0.01)$.

For all other physiological measurements, a one-way ANOVA was used to compare the control and two treatments, followed by a Tukeys post hoc analysis $(\alpha=0.05)$. ANOVA assumptions were tested using the Shapiro-Wilk and Kolmogornov-Smirnov normality tests, and the Brow-Forsythe test of equal variance. If violated, values were $\log _{10}$ normalised. Bonferroni adjusted P-values are presented to avoid type 1 errors. Data points that were less or more than two standard deviations of the average were removed as outliers. Details of statistical tests are in appendix section A1.2.

\subsection{Results}

\subsubsection{Chlorophyll fluorescence measurements}

\section{Rapid temperature increase}

The rapid increase in temperature from $25^{\circ} \mathrm{C}$ to $35^{\circ} \mathrm{C}$ had a minimal effect on the photochemistry of NZ01 (Figure 2.4 A\&B), but a dramatic photo-inhibitory effect on FLAp2 (Figure 2.4 C\&D). In the case of NZ01, the effective and maximum quantum yields decline slightly relative to controls at $35{ }^{\circ} \mathrm{C}$ by $9-15 \%$ (Unpaired T-test, $\mathrm{p}<0.0001$ for all significant differences from day 22 onwards). However, these values remained within the healthy range ( 0.45 and above), indicating that no severe photoinhibition was occurring.

In comparison to NZ01, the effective and maximum quantum yield of FLAp2 declined markedly in response to the rapid temperature increase (Figure $2.4 \mathrm{C} \& \mathrm{D}$ ). After $24 \mathrm{~h}$ and $48 \mathrm{~h}$ at $35^{\circ} \mathrm{C}$, both the effective and maximum quantum yield dropped by 46-58\% compared to the controls (Unpaired Ttest, $\mathrm{p}<0.00001$ for all). As these values were far below the lower limit of the healthy range (0.45), the treatment cultures were evidently experiencing severe photo-inhibition. 
A)

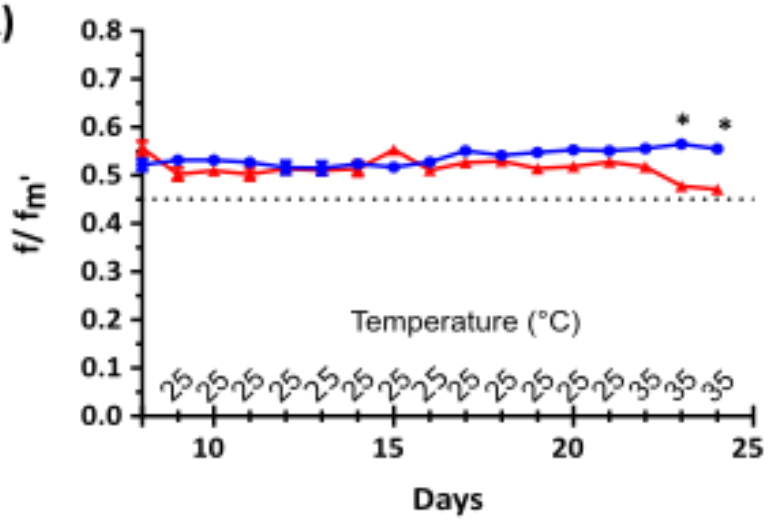

C)

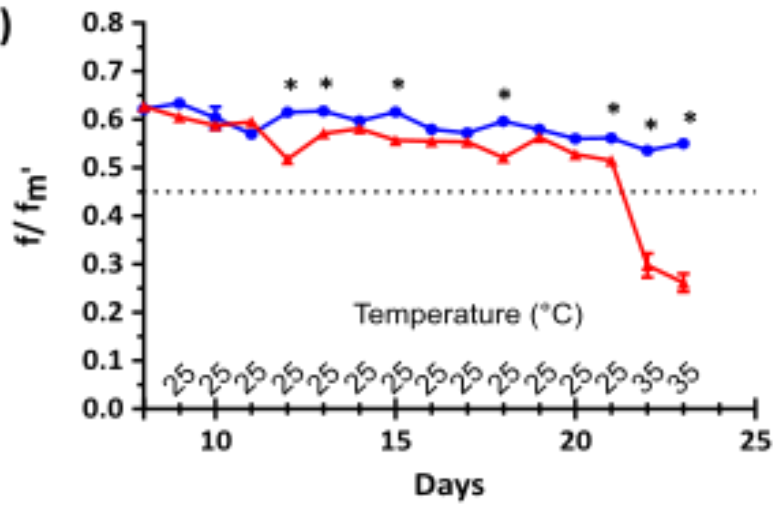

$\rightarrow$ Control
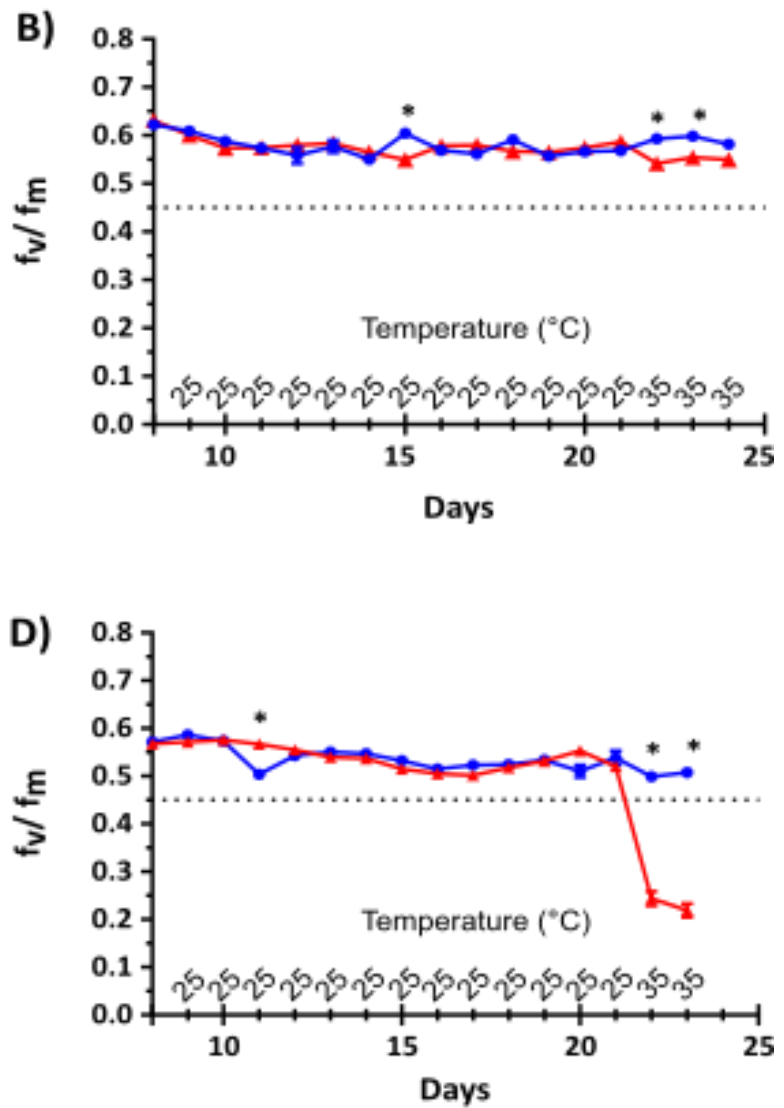

Rapid temperature increase treatment

Figure 2.4: Light and dark-adapted chlorophyll fluorescence measurements of two strains of $B$. minutum at different temperatures. A) Effective quantum yield $\left(\mathrm{f}_{\mathrm{v}} / \mathrm{f}_{\mathrm{m}}\right)$ for NZ01 $(\mathrm{n}=14)$. B) Maximum quantum yield $\left(f_{v} / f_{m}\right)$ after 20 minutes of dark acclimation for NZ01 $(n=14)$. C) Effective quantum yield $\left(f_{v} / f_{m}\right)$ for FLAp2 $(n=16)$. D) Maximum quantum yield $\left(f_{v} / f_{m}\right)$ after 20 minutes of dark acclimation for FLAp2 $(\mathrm{n}=16)$. Control cultures (blue) were maintained at $25^{\circ} \mathrm{C}$ for the entire experimental period. Treatment cultures (red), were increased from $25^{\circ} \mathrm{C}$ to $35^{\circ} \mathrm{C}$ on day 21 , as indicated by the $\mathrm{x}$ axis. Values presented are the mean $+/$ - standard error; if error bars are not visible, they are smaller than the data point. The dotted line indicates the value above which yield measurements are considered to be within the 'healthy' range ${ }^{1}$. Statistically significant differences between the control and treatment measurements on a given day are indicated by an asterix (Unpaired two tailed T-test, not assuming constant variance, details in appendix section A1.2, alpha $=0.01$ ).

\footnotetext{
${ }^{1}$ Generally, quantum yield values greater than 0.5 are considered in the healthy range (Gorbunov et al. 2001). A value of 0.45 was chosen to take a conservative approach.
} 


\section{Slow temperature increase}

Following the trend observed with those cultures exposed to a rapid temperature increase, a slow increase in temperature from $25^{\circ} \mathrm{C}$ to $35^{\circ} \mathrm{C}$ had a minimal effect on the photosynthetic health of NZ01 (Figure 2.5 A\&B), and an inhibitory effect on the photosystems of FlAp2 (Figure 2.5 C\&D). For NZ01, the effective quantum yield measurements were below that of the control for the entire experimental period, and so could not be directly compared to the control (Figure $2.5 \mathrm{~A}$ ). Thus, a repeated-measures ANOVA was performed, to compare how both the control and treatment measurements changed over time, finding no effect of elevated temperature on the effective quantum yield of NZ01 cultures. Alternately, the maximum quantum yield measurements of NZ01 showed a trend towards decline at elevated temperature by up to $15 \%$, however, no progressive decline was observed (see appendix A1.2 for t-test results) (Figure 2.5 B).

In comparison to NZ01, the chlorophyll fluorescence measurements for FLAp2 dropped dramatically at elevated temperature. Both the effective and maximum quantum yield progressively declined as the temperature increased (Figure 2.5 C\&D). The effective quantum yield of the treatment cultures was significantly lower than for the control cultures at $29^{\circ} \mathrm{C}$ (Unpaired T-test, $\mathrm{P}=0.002$ ), however this difference was not highly significant $(\mathrm{P}<0.0001)$ until the treatment cultures reached $30.2^{\circ} \mathrm{C}$ and above (Figure 2.5 C). Similarly, for the maximum quantum yield, there is a significant difference between the control and treatment cultures at $29^{\circ} \mathrm{C}$ and higher. However, this difference was not highly significant $(\mathrm{P}<0.0001)$ until $31.4^{\circ} \mathrm{C}$, the same temperature that the yield measurements dropped below 0.45 into the unhealthy range, indicating thermally-induced photo-inhibition. 

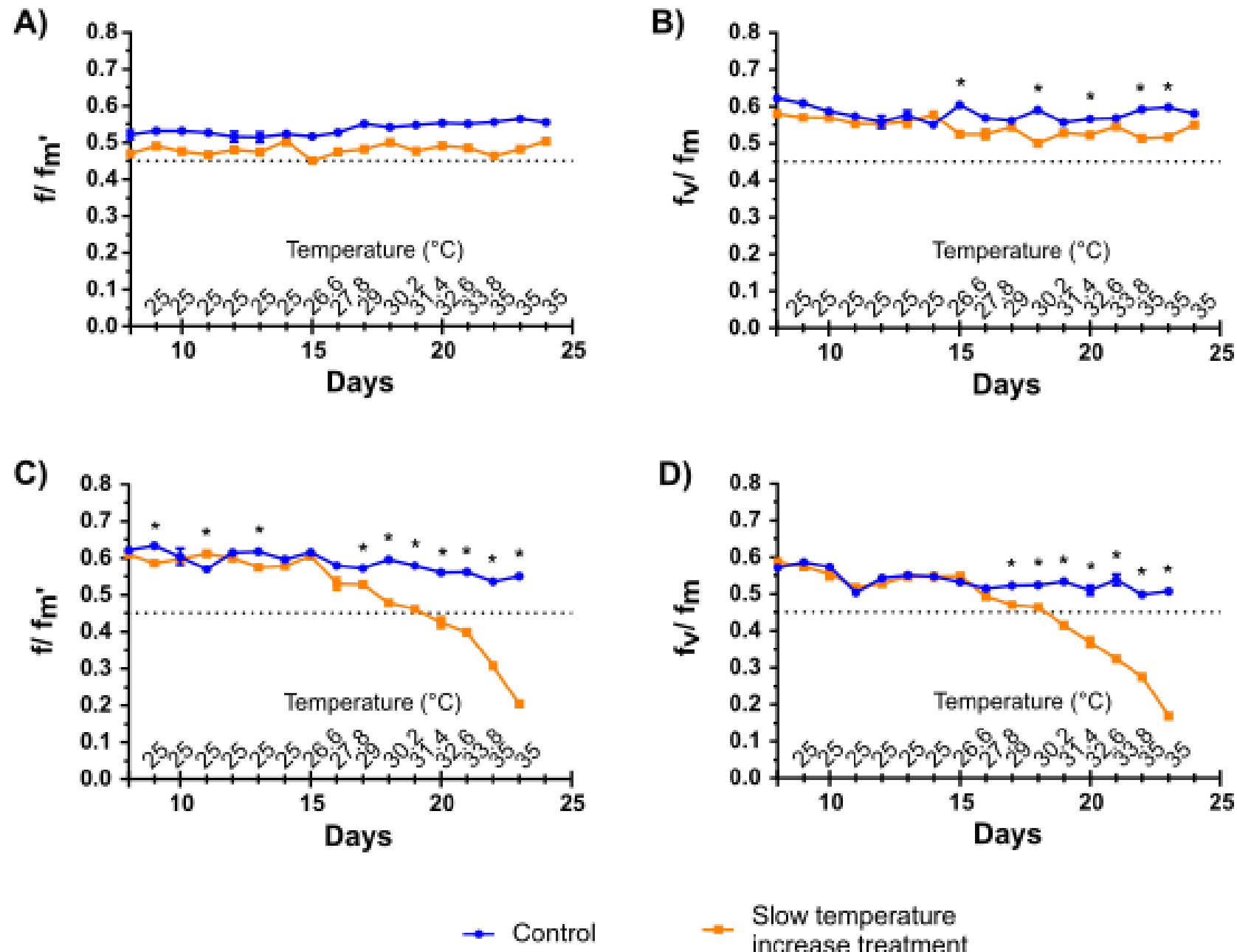

Slow temperature
increase treatment

Figure 2.5: Light and dark-adapted chlorophyll fluorescence measurements of two strains of $B$. minutum cultures at different temperatures. A) Effective quantum yield $\left(\mathrm{f}_{\mathrm{v}} / \mathrm{f}_{\mathrm{m}}\right)$ for NZ01 $(\mathrm{n}=14)$. B) Maximum quantum yield $\left(\mathrm{f}_{\mathrm{v}} / \mathrm{f}_{\mathrm{m}}\right)$ after 20 minutes of dark acclimation for NZ01 $(\mathrm{n}=14)$. C) Effective quantum yield $\left(\mathrm{f}_{\mathrm{v}} / \mathrm{f}_{\mathrm{m}}\right)$ for FLAp2 $(\mathrm{n}=16)$. D) Maximum quantum yield $\left(\mathrm{f}_{\mathrm{v}} / \mathrm{f}_{\mathrm{m}}\right)$ after 20 minutes of dark acclimation for FLAp2 $(\mathrm{n}=16)$. Control cultures (blue) were maintained at $25^{\circ} \mathrm{C}$ for the entire experimental period. Treatment cultures (orange) were slowly taken from $25^{\circ} \mathrm{C}$ to $35^{\circ} \mathrm{C}$ over a one week period as indicated by the $\mathrm{x}$-axis. Values presented are the mean $+/$ - the standard error; if error bars are not visible they are smaller than the data point. The dotted line indicates the value above which yield measurements are considered within the healthy range. Statistically significant differences between the control and treatment culture measurements are indicated by an asterisk (Unpaired two tailed T-test, not assuming constant variance, details in appendix section A1.2, alpha = 0.01 ). 


\subsubsection{Pigment concentration}

NZ01 and FLAp2 have different pigment profiles. Overall, FLAp2 had a higher pigment concentration than NZ01. This is especially evident when comparing the concentration of carotenoids between the two strains (Figure 2.6 A\&B), where FlAp2 concentrations are up to 5.8 times higher. This pattern is also reflected by a higher ratio of carotenoids to chlorophyll $a$ for FlAp2 compared with NZ01 across all treatments (Figure 2.6 C vs.D). Unsurprisingly, this aligns with the visual colour of the two cultures displayed in Figure 2.3, where FLAp2 has a more brownish tinge (the colour of peridinin) and NZ01 has a more greenish tinge (the colour of chlorophyll $a$ ).

The chlorophyll $a$ concentration of NZ01 was not significantly different between the controls and either of the elevated temperature treatments (ANOVA, $\mathrm{f}(2,6)=3.5, \mathrm{p}=0.0970)$ (Figure $2.6 \mathrm{~A}$ ). Conversely, temperature had a significant effect on the concentration of carotenoids (ANOVA, $\mathrm{f}(2,6)=6.4, \mathrm{p}=0.033)($ Figure 2.6 A), with those cells exposed to a rapid increase in temperature having a 2.6 times higher concentration than the control cultures (Tukey post hoc, $\mathrm{p}=0.0418$ ). The concentration of carotenoids in the cultures exposed to a slow increase in temperature was also higher than the control cultures on average but was not significantly different to either the control cultures or those exposed to rapid temperature increase. Although there appears to be a trend of an increase in the concentration of carotenoids in the treatment cultures compared to the controls, graph C illustrates that this is not paired with a concomitant down-regulation of chlorophyll $a$, as there is no significant difference between the carotenoid to chlorophyll $a$ ratio of the control and treatment cultures $($ ANOVA, $\mathrm{f}(2,6)=0.3, \mathrm{p}=0.778)($ Figure 2.6 C).

For FLAp2, both the chlorophyll $a$ concentration (ANOVA, $\mathrm{f}(2,9)=3.5, \mathrm{p}=0.0970$ ), and the concentration of carotenoids (ANOVA, $\mathrm{f}(2,9)=2.9, \mathrm{p}=0.101$ ), were not significantly different between the controls and either of the elevated temperature treatments (Figure 2.6 B). Unsurprisingly, this is also reflected in the ratio of carotenoids to chlorophyll $a$, for which there was also no significant difference between the control and treatment cultures (ANOVA, $f(2,9)=0.85, p=$ 0.463)(Figure 2.6 D). 
A)

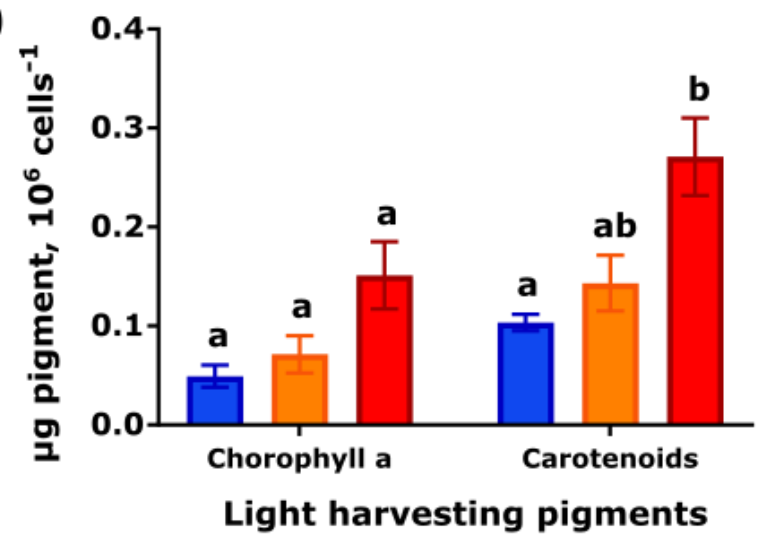

C)

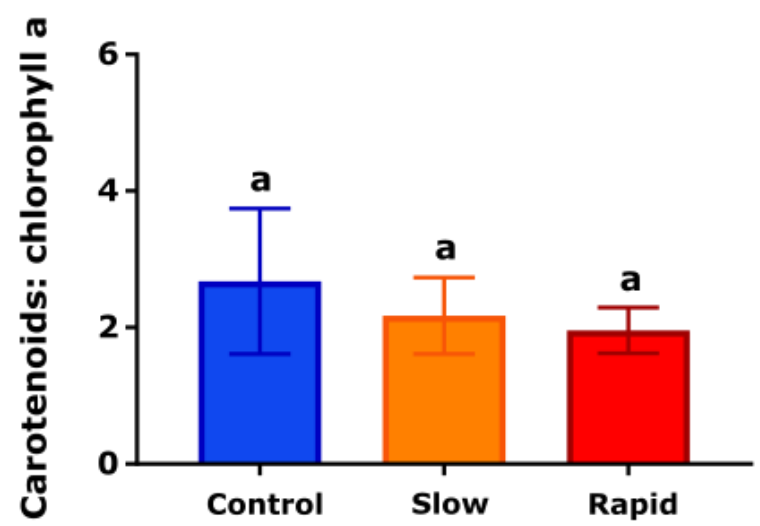

Control
B)

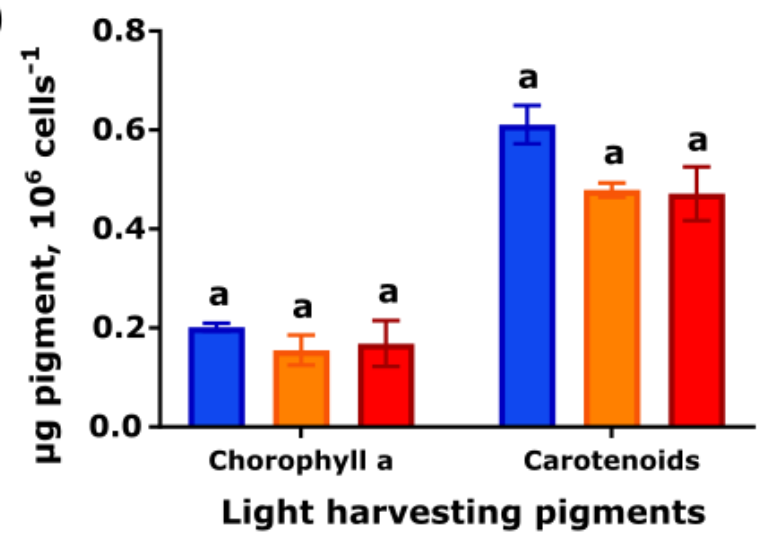

D)

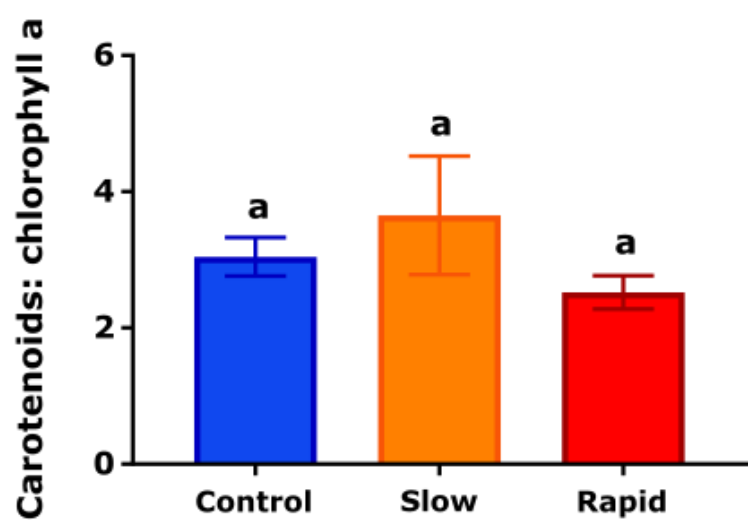

Slow temperature increase
Rapid temperature

increase

Figure 2.6: The concentration of chlorophyll $a$ and 'carotenoids (peridinin and xanthophylls). A) NZ01 $(\mathrm{n}=3)$. B) FLAp2 $(\mathrm{n}=4)$. C) NZ01 ratio of carotenoids to chlorophyll $a(\mathrm{n}=3)$. D) FLAp2 ratio of carotenoids to chlorophyll $a(\mathrm{n}=4)$. Values presented are the mean $+/$ - standard error. The letters indicate whether values are significantly different (ANOVA and Tukey post hoc, alpha $=0.05$, statistical tests in appendix section A1.2). 


\subsubsection{Photosynthetic and respiratory oxygen flux}

Oxygen flux measurements provided insight into the effect of elevated temperature on the metabolic state of the algal cultures, in terms of how much oxygen is produced and hence how much organic carbon is fixed in photosynthesis, compared to how much is being consumed by respiratory activity (i.e. gross photosynthesis: respiration; P:R).

\section{NZ01}

Elevated temperature did not have a negative effect on the metabolic health of NZ01. There was no significant difference between the rates of respiration, net photosynthesis or gross photosynthesis, or $\mathrm{P}: \mathrm{R}$, of the control cultures and those subject to a slow increase in temperature (Figure $2.7 \mathrm{~A}-\mathrm{D}$ ). Conversely, the respiration rate, as well as gross photosynthetic rate of the cultures exposed to a rapid increase in temperature were at least double those of both the control cultures and those subjected to a slow increase in temperature (Tukey post hoc, $\mathrm{P} \leq 0.0003$ for all comparisons). These thermal effects on photosynthesis and respiration were of a similar magnitude however, so there was no significant difference between P:R ratios of control and treatment cultures (ANOVA, $f(2,9)=0.088, p=0.917$ ).

\section{FlAp2}

FLAp2 showed a general trend of decreased metabolic health at $35^{\circ} \mathrm{C}$ compared to $25^{\circ} \mathrm{C}$ (Figure 2.8 D). In comparison to controls, respiration rates were elevated by 39 and $65 \%$ for cultures exposed to a slow, or rapid increase in temperature, respectively. However, this difference was only significantly different for the rapid temperature increase (Figure $2.8 \mathrm{~B}$; Tukey post hoc, $\mathrm{p}=0.0166$ ). The gross photosynthetic rate of control cultures was 72 and $47 \%$ higher than the rates of cultures exposed to a slow and rapid increase in temperature, respectively (graph A; Tukey post hoc $\mathrm{p}=0.0001$ and $\mathrm{p}=0.02$ ). Interestingly, gross photosynthetic rates were $47 \%$ lower for cultures under a slow versus rapid temperature increase (graph A; Tukey post hoc, $\mathrm{p}=0.01$ ). This is in alignment with the chlorophyll fluorescence measurements (Section 2.3.1.), which indicated that thermally-induced photoinhibition was marginally less for those cultures exposed to a rapid versus slow temperature increase. Overall, in terms of metabolic balance, the $\mathrm{P}: \mathrm{R}$ ratio indicates that the cultures kept at control temperatures were healthiest, being $80 \%$ and $67 \%$ greater than the cultures exposed to and slow and rapid temperature increase, respectively (graph $\mathrm{C}$; Tukey post hoc, $\mathrm{p}=0.001, \mathrm{p}=0.02$, respectively). Notably, the P:R ratio for cultures at elevated temperature was $<1$, indicating that more organic carbon was being consumed by respiratory activity than produced by photosynthesis. 
A)

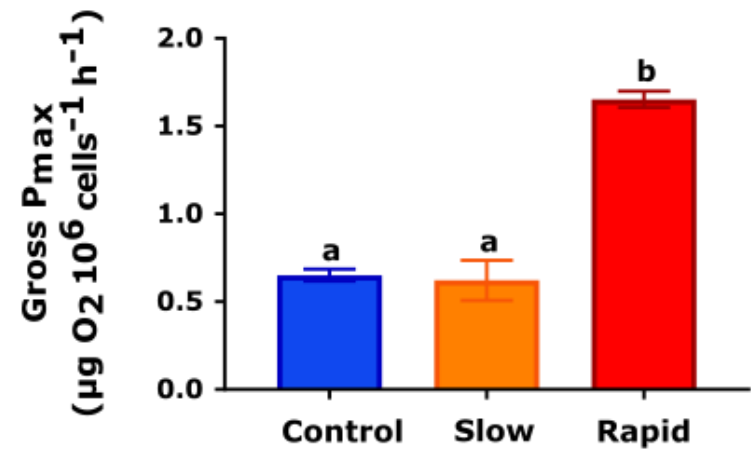

B)

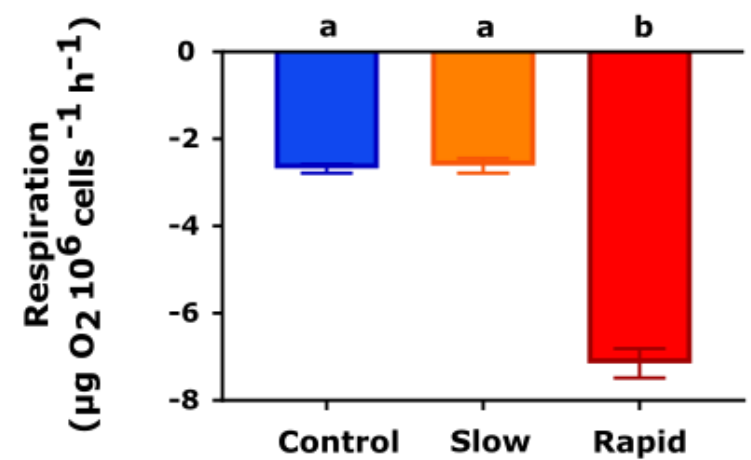

C)

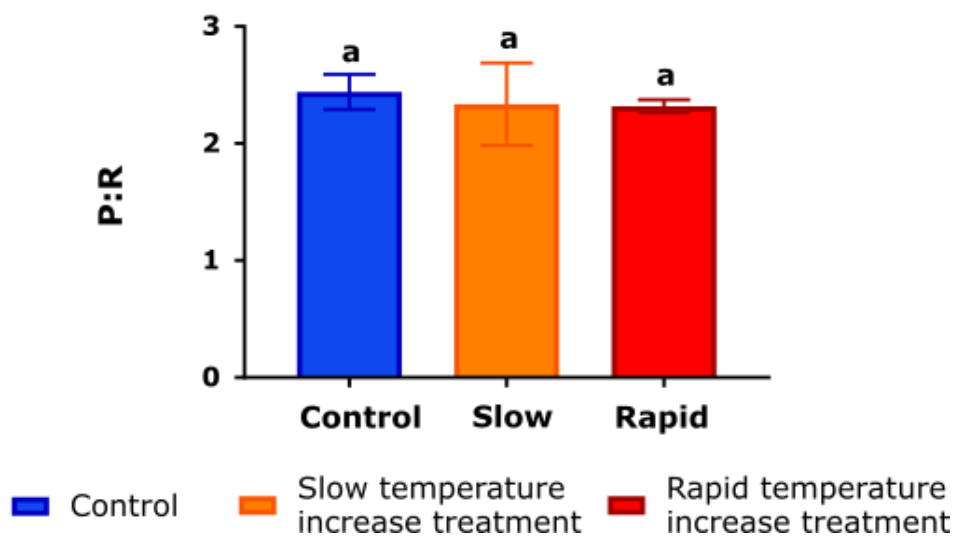

Figure 2.7: Maximum rates of oxygen evolution and consumption in NZ01 cultures exposed to different temperatures, normalised to cell density. A) Respiration; B) Gross photosynthesis; C) Ratio of gross photosynthesis to respiration (P:R). Values presented are the mean $+/$ - standard error. The letters indicate whether values are significantly different (ANOVA and Tukey post hoc, alpha $=$ 0.05 , statistical tests in appendix section A1.2). 
A)

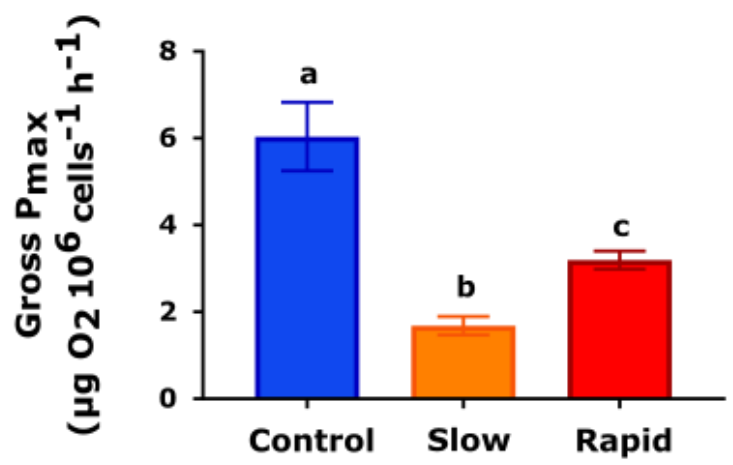

B)

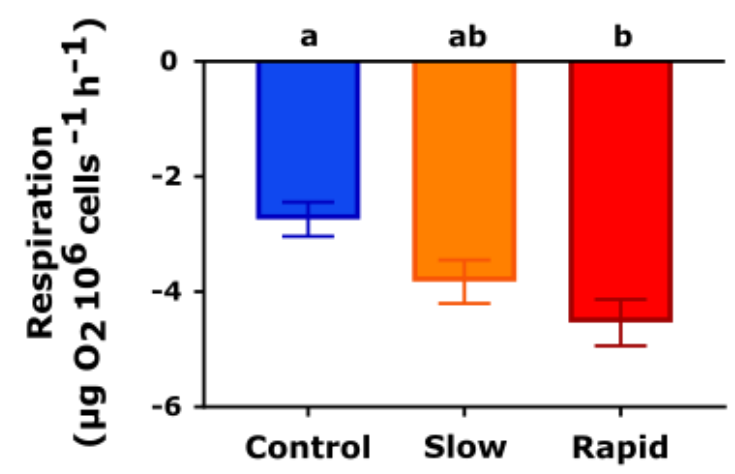

C)

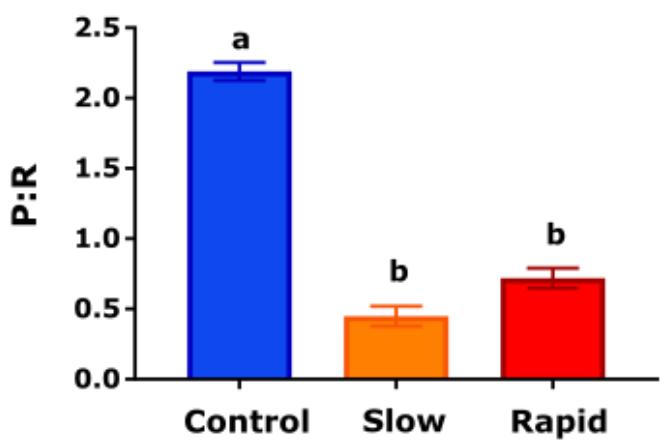

Contro

$\square \begin{aligned} & \text { Slow temperature } \\ & \text { increase treatment }\end{aligned} \quad \begin{aligned} & \text { Rapid temperature } \\ & \text { increase treatment }\end{aligned}$

Figure 2.8: Maximum rates of oxygen evolution and consumption in FLAp2 cultures exposed to different temperatures, normalised to cell density. A) Maximum respiration rate; B) maximum gross photosynthetic rate; $\mathrm{C}$ ) the ratio of maximal gross photosynthesis to respiration, these values were $\log _{10}$ transformed to meet ANOVA assumptions of normality. Values presented are the mean $+/-$ the standard error. The letters indicate whether values are significantly different (ANOVA and Tukey post hoc, alpha $=0.05$, statistical tests in appendix section A1.2) 


\subsubsection{Population growth rate}

Elevated temperature had a negative effect on the population growth of NZ01. The percentage increase in cell density of NZ01 cultures over the experimental period was significantly different between the treatments and the control, as well as between the treatments (ANOVA, $\mathrm{f}(2,39)=56.57$, $\mathrm{p}<0.0001$ ) (Figure 2.9 A). The mean percent increase in cell density for the control was 1.5 times higher than that of the cultures exposed to a slow temperature increase (Tukey post hoc, $\mathrm{p}<0.0001$ ), and 4.3 times higher than the cultures exposed to a rapid temperature increase (Tukey post hoc, $\mathrm{p}=$ $0.003)$.

In comparison to NZ01, FLAp2 exhibited a 62-68\% decrease in cell density over the experimental period for the control and treatment cultures, with no significant difference between groups (ANOVA, $\mathrm{f}(2,45)=2.5, \mathrm{p}=0.0934$ ) (Figure $2.9 \mathrm{~B}$ ). This could partially be because of cell death but is likely largely attributed to the propensity of FLAp2 to form mats on the surface of the plastic tube in which they were contained. Despite efforts to wash the algae from their tubes, algae not visible to the human eye could have remained. Previous published studies have also had the issue of cell adhesion to culture tubes affecting growth rate calculations (Krämer et al. 2012, Karim et al. 2015). Because of this discrepancy no inferences can be made on the effect of thermal stress on the growth rates of the FLAp2 cultures, and thus this metric is not discussed.

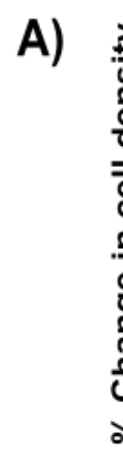

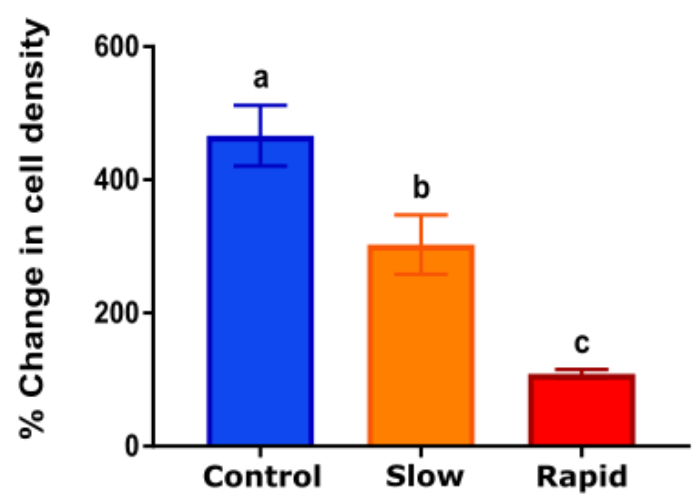

Control
B)

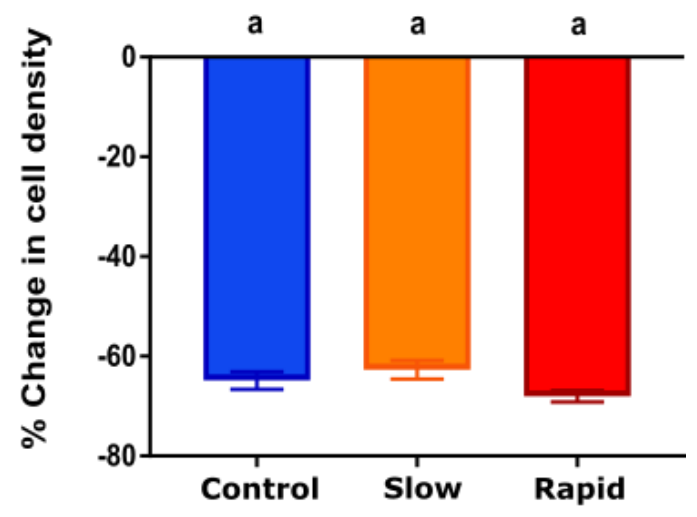

Slow temperature increase
Rapid temperature increase

Figure 2.9: The percentage difference in the cell density of cultures from the beginning of the experimental period to the end of the experimental period. A) NZ01 $(n=14)$. B) FLAp2 $(n=16)$. Values presented are the mean $+/$ - the standard error. The letters indicate whether values are significantly different (ANOVA and Tukey post hoc, alpha $=0.05$, statistical tests in appendix section A1.2). 


\subsubsection{Alkaline phosphatase activity}

NZ01 exhibited a general trend of increased alkaline phosphatase activity at elevated temperature (ANOVA, $\mathrm{f}(2,6)=7.1, \mathrm{p}=0.027)($ Figure $2.10 \mathrm{~A})$. The mean APA activity of the cultures exposed to a slow increase in temperature was 3.4 times higher than that of the control cultures (Tukey post hoc, $\mathrm{p}=0.0228$ ). Similarly, the APA activity for cultures exposed to a rapid increase in temperature was also higher than that of the controls, by an average of 1.4 times, however this difference was not significant due to variance (Tukey post hoc, $\mathrm{p}=0.1346$ ). The trend was very different for FLAp2 $($ ANOVA, $\mathrm{f}(2,6)=5.83, \mathrm{p}=0.039)($ Figure $2.10 \mathrm{~B})$. The control cultures had very similar levels of APA activity to those cultures exposed to a slow increase in temperature. In comparison, the mean APA activity in the cultures exposed to a rapid increase in temperature was approximately half of what was observed for the control cultures (Tukey post hoc, $\mathrm{p}=0.0317$ ), and those exposed to a slow temperature increase (Tukey post hoc, $\mathrm{p}=0.0211$ ).

\section{A)}

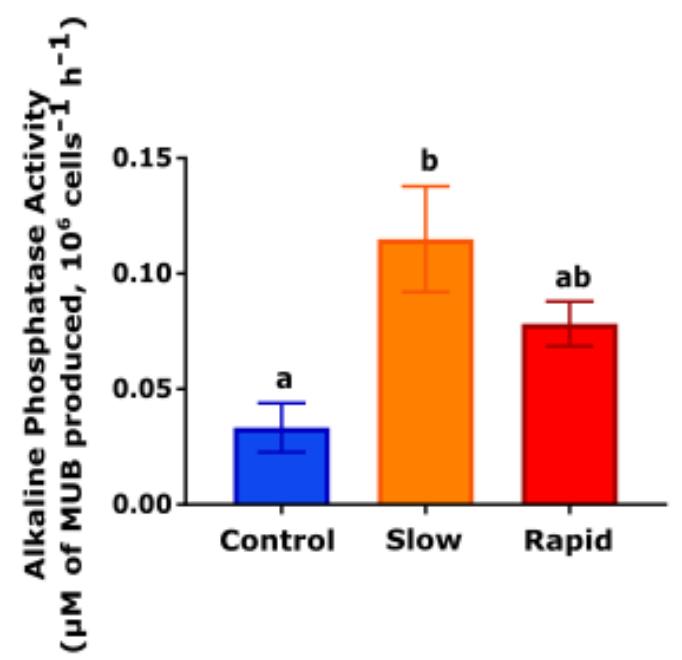

Control
B)

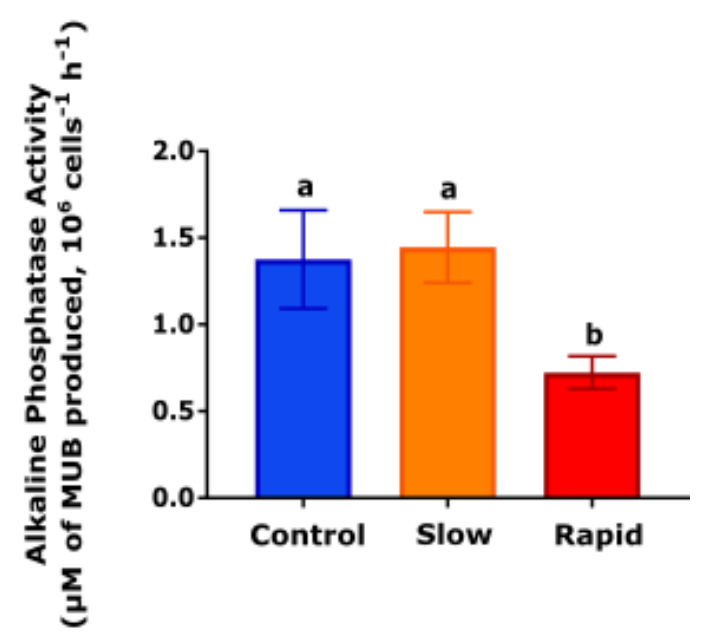

Rapid temperature increase

Figure 2.10: Alkaline phosphatase activity of cultures that were exposed to different temperature treatments. A) NZ01 $(n=3)$. B) FLAp2 $(n=3)$. Values presented are the mean $+/$ - standard error. All values across graphs A and B were $\log _{10}$ transformed to meet ANOVA assumptions of normality. The letters indicate whether values are significantly different (ANOVA and Tukey post hoc, alpha = 0.05, statistical tests in appendix section A1.2). 


\subsection{Discussion}

\subsubsection{Overview}

In summary, despite being genetically identified as the same species, the NZ01 and FlAp2 strains of B. minutum have different thermal tolerances. NZ01 showed greater physiological adaptability and was able to maintain healthy photosynthetic function and metabolic balance at $35^{\circ} \mathrm{C}$. Alternately, FLAp2 exhibited severe photoinhibition and reduced metabolic health at $35^{\circ} \mathrm{C}$. The different heating rates used in this study highlight the functional variation between strains. Interestingly, FlAp2 showed more significant thermal stress at a slower heating rate, exemplified by reduced photosynthetic rates relative cultures exposed to a rapid temperature increase. Alternately, NZ01 cultures exposed to a slow versus rapid heating rate demonstrated greater thermal acclimation, as alkaline phosphatase activity was higher, and unlike cultures exposed to a rapid temperature increase, respiration and gross photosynthetic rates were equal to cultures at control temperatures.

\subsubsection{Thermal effects on the photosystems of Breviolum minutum}

\section{Chlorophyll fluorescence measurements}

Chlorophyll fluorescence provides an overview of photosynthetic health (Lichtenthaler 1988, Maxwell and Johnson 2000), with reduction in quantum yield indicative of photosynthetic dysfunction (Genty et al., 1989; Warner et al., 1999). Among other physiological measurements, quantum yield has been repeatedly used as an indicator of differential thermal tolerance between Symbiodiniaceae taxa (Tchernov et al. 2004, Fisher et al. 2012, Krueger et al. 2014). In this study, the quantum yield values indicate that strain NZ01 has a much greater capacity to maintain photosynthetic function at elevated temperature than strain FLAp2. The effective and maximum quantum yield measurements of NZ01 dropped marginally in response to elevated temperature, possibly reflecting a slight down-regulation of photosystem II (PSII), to avoid over excitation (Hill et al. 2005, Gururani et al. 2015, Warner and Suggett 2016). Alternately, in the case of FLAp2, there was a dramatic drop in chlorophyll fluorescence in response to elevated temperature. Quantum yield measurements have been observed to drop in response to elevated temperatures for Symbiodiniaceae both in hospite and in culture, under both laboratory or field conditions (Hoegh-Guldberg and Smith 1989b, Iglesias-Prieto et al. 1992, Fitt and Warner 1995, Warner et al. 1996, Jones et al. 2000, Bhagooli and Hidaka 2003, Hill et al. 2004). This indicates a down-regulation of photosystem II as 
well as severe thermally-induced damage and dysfunction of photosystem II (Nishiyama and Murata 2014, Warner and Suggett 2016) Significantly, when PSII is severely damaged it is no longer able to dissipate photosynthetic energy as heat (non-photochemical quenching; NPQ) (Gorbunov et al. 2001) resulting in over-excitation of the photosynthetic apparatus and the production of ROS (Bhagooli and Hidaka 2003, Lesser 2006, Rehman et al. 2016). Photo-inhibition of photosystem II has been observed to precede thermal bleaching (Hoegh-Guldberg and Smith 1989a, Lesser 1997, Hillyer et al. 2017), so it is widely assumed that this dysfunction is linked to the initiation of the cnidarian bleaching response (Baird et al. 2009). Therefore, in the context of the cnidarian-dinoflagellate symbiosis, the NZ01 strain of Breviolum minutum may be a more beneficial symbiont at elevated temperatures than the FLAp2 strain.

Interestingly, for NZ01 the maximum quantum yield measurements were on average $9 \%$ higher than the effective quantum yield measurements, whereas for FLAp2 they were 8\% lower (see Figures 2.4 $\& 2.5)$. Generally, the dark-adapted maximum quantum yield is expected to be higher than the lightadapted quantum yield, as in darkness the plastoquinone (PQ) pool becomes fully oxidised, and nonphotochemical quenching mechanisms are switched off in the absence of light (Schreiber 2004). However, the discrepancy observed in FLAp2 has been observed in previous studies measuring the chlorophyll fluorescence of Symbiodiniaceae in situ and has been linked to the reduction of the PQ pool due to chlororespiration (Jones and Hoegh-Guldberg 2001, Ulstrup et al. 2005, Hill and Ralph 2008). Chlororespiratory activity can be different between Symbiodiniaceae genera (Reynolds et al. 2008, Roberty et al. 2014, Aihara et al. 2016), and provides a possible mechanism behind the discrepancy in light and dark-adapted chlorophyll fluorescence measurements observed between strains here.

\section{Pigment Concentration}

Photobleaching, or a reduction in pigment concentration, has been observed in Symbiodiniaceae in response to elevated temperature both in culture and in hospite (Porter et al. 1989, Warner et al. 1996, Takahashi et al. 2004, 2008). Photobleaching follows photoinhibtion and is primarily a result of the suppression of photosystem repair mechanisms, and the de novo synthesis of antenna proteins (Warner et al. 1999, Takahashi et al. 2004, 2008). This response was not observed for either NZ01 or FLAp2. Consistent with this study, other experiments have also observed no change in the chlorophyll $a$ concentration when Symbiodiniaceae are exposed to thermal stress, despite a drop in the quantum yield (Iglesias-Prieto et al. 1992, Rowan 2004, Hillyer et al. 2016b). This could reflect 
that photosystem repair mechanisms are still functional despite thermal stress. In addition, energy can also be invested in photoprotection (Ragni et al. 2010), whereby the light energy absorbed by the pigments may be quenched by non-photochemical mechanisms to reduce the over-excitation of photosystem II and potential damage (Gorbunov et al. 2001). This could explain the significant increase in the concentration of carotenoids observed for NZ01 when exposed to a rapid increase in temperature, as not all carotenoids are photoactive and are instead photoprotective (Young 1991). Interestingly, when NZ01 was exposed to a slow increase in temperature, it did not show the same response, indicating that this change in pigment profile may be an initial response to thermal stress. These results demonstrate the greater physiological plasticity of NZ01 than FLAp2.

\subsubsection{Thermal effects on metabolic balance and growth}

\section{Oxygen flux measurements}

Oxygen flux measurements provided insight into the metabolic state of the algal cultures, in terms of how much oxygen is produced and hence how much organic carbon is fixed in photosynthesis, compared to how much is being consumed by respiratory activity. Consistent with the chlorophyll fluorescence measurements (see above), NZ01 exhibited no negative response to elevated temperature with respect to either gross photosynthesis or metabolic balance (P:R ratio), irrespective of thermal treatment. Interestingly, in the case of NZ01 exposed to a rapid increase in temperature both the respiration and photosynthetic rates were at least double those of both the control cultures and those exposed to a slow temperature increase. A similar response has also been observed in Breviolum minutum isolated from the anemone Condylactis gigantea, however temperatures were only elevated from $17.8^{\circ} \mathrm{C}$ to $26.8^{\circ} \mathrm{C}$ (as opposed to 25 to $35^{\circ} \mathrm{C}$ in this study) (Karako-Lampert et al. 2005). This could reflect an increase in the rate of electron transport with temperature (Falkowski \& Raven, 2007), along with the intrinsic link between the reaction rates of photosynthesis and respiration, as the products of one process are used as reactants for the other process (Kromer et al. 1993, Kromer 1995, Hoefnagel et al. 1998, Noguchi and Yoshida 2008). For example, mitochondrial respiration rate has been shown to increase in response to photosynthetically produced oxygen in both marine algae and Symbiodiniaceae in hospite (Dromgoole 1978, Harland and Davies 1995). NZ01 at control temperatures and those exposed to a slow temperature increase had equal respiration and gross photosynthetic rates, reflecting acclimation to elevated temperature. As reaction rates are expected to increase with temperature (Peterson et al. 2007), this may reflect a down-regulation of photosynthetic and respiratory reactions to avoid potential over-excitation and damage at elevated temperature (Berry 
and Bjorkman 1980, Hikosaka et al. 2005, Eberhard et al. 2008, Takahashi and Badger 2011, Takahashi et al. 2013). A previous study comparing the effect of heating rate on oxygen evolution per symbiont in hospite, found that while oxygen evolution dropped compared to controls at $30-33^{\circ} \mathrm{C}$, this drop was less pronounced for symbionts exposed to a heating rate of $0.5^{\circ} \mathrm{C}$ per day as opposed to $1^{\circ} \mathrm{C}$ per day (Middlebrook et al. 2010). While clearly this symbiont type (not specified in paper), illustrated less physiological adaptability than NZ01, this example also demonstrates a possible acclimation response to elevated temperature at a slower heating rate.

Unlike NZ01, FLAp2 exhibited decreased metabolic health when exposed to elevated temperature. Whether exposed to either a slow or rapid increase in temperature, this strain exhibited a reduced rate of gross photosynthesis and an increased rate of respiration, which is typical of Symbiodiniaceae both in culture and in hospite under thermal stress (Iglesias-Prieto et al. 1992, Buxton et al. 2009, Oakley et al. 2014b). Interestingly, the gross photosynthetic rate of FlAp2 was lower for cultures exposed to a slow versus rapid temperature increase. Due to the severe photo-inhibition observed for FlAp2 at $35^{\circ} \mathrm{C}$, it is likely that the maximum thermal tolerance of this strain is lower than $35^{\circ} \mathrm{C}$ (likely between $31-32^{\circ} \mathrm{C}$, see Figure 2.5). Thus, cultures exposed to a slow temperature increase have been above this tolerance level for longer than rapidly heated cultures, and so were experiencing greater photoinhibition. As for NZ01, both gross photosynthesis and respiration rates were higher for the rapid versus slow temperature increase, but respiration increased to a proportionally greater extent meaning that P:R declined relative to the control, indicating reduced metabolic health. A reduced P:R has been observed repeatedly in thermally-stressed Symbiodiniaceae both in culture and in symbiosis (Coles and Jokiel 1977, Rowan 2004, Goulet et al. 2005, Oakley et al. 2014b), and reflects increased energy expenditure in order to maintain cellular function while under thermal stress (Kaplan et al. 2004). Specifically, as the P:R ratio was bellow one for treatment cultures, these cells are in a state of negative metabolic balance and would eventually die if left at $35^{\circ} \mathrm{C}$. In conjunction with the chlorophyll fluorescence measurements, the oxygen flux measurements demonstrate the greater thermal tolerance of NZ01 than FLAp2.

\section{Population growth rates}

While no inferences can be made about the effect of elevated temperature on the growth rate of FLAp2 due to the methodological limitation described earlier, NZ01 showed an interesting trend of reduced population growth with increased temperature, with this trend being significantly stronger for those cultures exposed to a rapid versus slow temperature increase. The thermal optimum for growth 
is typically lower than that for photosynthesis (Davison 1991), which may explain the observed increase in gross photosynthetic rate in conjunction with decreased growth in NZ01 when exposed to a rapid temperature increase. Similarly, decreased cellular growth in conjunction with minimal declines in $F_{\mathrm{v}} / F_{\mathrm{m}}$ has been described for other Symbiodiniaceae taxa in response to elevated temperature (Karim et al. 2015). This signifies an uncoupling between photosynthesis and growth, and is likely due to respiratory losses and the energy demands of maintaining protein repair and photo-physiological function at elevated temperature, which redirects energy away from cellular growth (Kaplan et al. 2004, Goulet et al. 2005, Robison and Warner 2006, Karim et al. 2015). It is worth noting that the cell counts to calculate population growth were taken at the beginning and end of the experimental period. Thus, those cultures exposed to a slow temperature increase may have had increased growth rates at moderately elevated temperatures, as catalysed reactions increase with temperature up to a point, after which thermal stress causes the denaturing of proteins and loss of functionality (Karako-Lampert et al. 2005, Peterson et al. 2007). This could explain the relatively higher population increase for these cultures.

\subsubsection{Nutritional response to thermal stress}

\section{Alkaline phosphatase activity assay}

Alkaline phosphatase activity in Symbiodiniaceae may increase in response to temperature, facilitating greater phosphorus availability. Phosphorus is an essential nutrient and is the key component in ATP, nucleotides and membrane phospholipids (Lehninger et al. 1993). Alkaline phosphatase converts organic phosphorus into inorganic phosphorus $\left(\mathrm{P}_{\mathrm{i}}\right)$, allowing the regeneration of $\mathrm{P}_{\mathrm{i}}$ in phosphate-deficient environments (Feder 1973), in addition to phosphorus uptake, as it must be in its inorganic form to pass through the cellular membrane (Doonan and Jensen 1980, Vincent and Crowder 1995, Ferrier-Pagès et al. 2016). Increased alkaline phosphatase activity has been used to identify phosphate deficiency in a variety of marine autotrophs, including benthic algae (Lapointe 1987, Lapointe and O'Connell 1989), sea grasses (Short et al. 1985, Pérez and Romero 1993), marine diatoms (Møller et al. 1975), and in Symbiodiniaceae (Annis and Cook 2002, Wiedenmann et al. 2013). However, no one has investigated the effect of temperature on the expression of this enzyme as performed here. NZ01 demonstrated higher APA in response to elevated temperature. However, this increase was only statistically significant between the control and those cells exposed to a slow increase in temperature. This may be because a slow increase in temperature allowed more time for possible epigenetic change and protein upregulation, whereas a rapid increase in temperature would 
not only provide less time for protein upregulation to occur, but may have induced cellular shock and the redirection of resources elsewhere (Kaplan et al. 2004). Previous studies have identified that increased phosphorus availability increases the thermal tolerance of Symbiodiniaceae in hospite (Ezzat et al. 2016a), and deficiency decreases thermal tolerance, possibly due to altered composition of the thylakoid membrane increasing vulnerability to damage (Béraud et al. 2013, Wiedenmann et al. 2013, Rosset et al. 2017). Thus, due to the integral part that phosphorus availability plays in thermal tolerance, it is logical that that an organism would upregulate the expression of alkaline phosphatase under thermal stress as was observed here.

Unlike NZ01, FLAp2 did not exhibit increased APA in response to elevated temperature. Conversely, APA activity for both the control and slow increase treatments was significantly higher than the activity when exposed to a rapid temperature increase. This possibly reflects a loss of enzyme function due to the rapid temperature change (Peterson et al. 2007), which was able to be mediated when temperature was increased more slowly. It is also notable that the alkaline phosphatase activity for FLAp2 is ten-fold higher than that of NZ01, which could relate to epigenetic differences between the two algal strains or be an artefact of experimental design, as the two assays were performed on separate days but using the same reagent stock solutions. The fact that, unlike NZ01, FlAp2 did not show increased APA in response to thermal stress further illustrates the greater physiological adaptability to thermal stress of NZ01.

The MUB assay used in this study has never been used for determining Symbiodiniaceae alkaline phosphatase activity. Being more sensitive than the PNPP assay which has previously been used (Annis and Cook 2002, Wiedenmann et al. 2013), this methodology could be utilised in future studies as a biomarker of phosphorus availability and deficiency on coral reefs (Annis and Cook 2002).

\subsubsection{Conclusion}

Though limited, previous studies have also identified functional differences between different genotypes or strains of the same Symbiodiniaceae species. For example, a transcriptomic study identified differential expression of genes between strains of B. minutum (mac703, Mf1.05b, rt002, rt351) (Parkinson et al. 2016). Specifically, there was differential expression in fatty acid metabolism genes, which is interesting as the composition of fatty acids which make up the thylakoid membrane in Symbiodiniaceae has been found to be a determinant of differential thermal tolerance between algal types (Tchernov et al. 2004, Díaz-Almeyda et al. 2011). Although not measured in this transcriptomic 
study, these differences in gene expression may confer differences in physiology and adaptability to environmental stressors between strains (Parkinson et al. 2016). This concept has been illustrated by observed differential thermal tolerance between populations of the same species (ITS2 type C1), both in hospite and in culture as a product of adaption to local environmental conditions (Howells et al. 2012). In addition, a very recently published paper observed different functional responses between different culture strains of $B$. minutum at 26 and $30^{\circ} \mathrm{C}$, and at different nitrate concentrations, however based on the results, no conclusions of difference in thermal tolerance between strains could be made (Bayliss et al.). This current study adds to the data supporting the intra-species physiological plasticity of the Symbiodiniaceae family, providing evidence of this phenomenon in strains which have not before been directly compared. This was emphasised by different heating rates; when exposed to a slow versus rapid heating rate the less tolerant strain showed more substantial photosynthetic dysfunction, while the more thermally tolerant strain demonstrated greater acclimation. Compared to a rapid heating rate, the slow temperature increase may have provided greater opportunity for NZ01 to adapt to $35^{\circ} \mathrm{C}$, possibly through protein upregulation and/or epigenetic change (Madlung and Comai 2004, Bruce et al. 2007, Edmunds and Gates 2008, Takahashi et al. 2013). This emphasises the importance of considering heating rate when performing thermal stress studies.

The functional differences between strains in their ability to maintain photosynthetic function and metabolic balance at elevated temperature, are characteristics that would likely confer differences in symbiosis stability with a cnidarian host under thermal stress. This possibility is exemplified by functional differences between specific host-symbiont genotypes within coral populations (Abrego et al. 2008, Parkinson and Baums 2014, Parkinson et al. 2015). However, the physiological responses of Symbiodiniaceae in culture may be different than when it is in hospite (Bhagooli and Hidaka 2003, Goulet et al. 2005, Buxton et al. 2009). Thus, the next step in this investigation would be to determine whether the dramatic differences in thermal tolerance between the NZ01 and FlAp2 strains of $B$. minutum are sustained in hospite. This study highlights the importance of characterising the physiological plasticity of Symbiodiniaceae on a population level, as this diversity may confer increased acclimatory capacity and thermal tolerance to the cnidarian-algal holobiont and contribute to the prediction and remediation of bleaching events.

In addition, to fully understand cnidarian bleaching we must further characterise the cellular mechanisms underlying the physiological response of Symbiodiniacae to thermal stress and other environmental factors. 'Omics' techniques have proven to be invaluable to this endeavour. Genomic, transcriptomic and metabolomic studies of Symbiodiniaceae have provided insight into the shifts in cellular processes that culminate in altered physiology (Meyer and Weis 2012). However, due to 
limitations such as posttranscriptional protein modification (Leggat et al. 2011), a complete picture of cellular ecophysiology is yet to be revealed. In the next chapter, novel proteomics methodology is applied for the first time to Symbiodiniaceae, in an effort to unravel the complexities of the cellular response to thermal stress and nutrient availability. 


\section{Chapter 3: The link between the nutrient environment and the thermal tolerance of Breviolum minutum}

\subsection{Introduction}

\subsubsection{Eutrophication of coastal ecosystems}

Half the human population lives within $60 \mathrm{~km}$ of the coast, a number only expected to increase as the human population grows (Tilman et al. 2001). Anthropogenic presence along the coastline has a dramatic influence on coastal ecosystems as the natural environment is altered and shaped to fit our needs. A significant effect of this anthropogenic activity is the input of nutrients into marine coastal ecosystems. Organic and inorganic nutrients dissolved in water, or within sediments or organic matter, enter the coastal system via aeolian dust or riverine and diffuse discharge (Brown 1997, Fabricius 2005, Wooldridge 2009, Brodie et al. 2010, Wagner et al. 2010, Brodie et al. 2012, Weber et al. 2012). The primary enrichers are nitrogen and phosphorus. However, the relative abundance of each of these nutrients depends on the source. Generally, runoff from urbanised areas and agricultural land results in nitrogen enrichment (Howarth et al. 1996, Carpenter et al. 1998). Alternately, municipal and industrial wastewater which enters coastal systems contains very high levels of phosphorus (Conley et al. 2009). This nutrient loading is dramatically influencing biogeochemical cycling in coastal environments, having reverberating effects on both the species and ecosystem levels (Rabalais et al. 2009, Howarth et al. 2011, Valiela et al. 2016).

\subsubsection{Coral reefs, coral bleaching and eutrophication}

An ecosystem particularly vulnerable to eutrophication is coral reefs, which typically thrive in oligotrophic tropical waters (Muscatine and Porter 1977). Worldwide, increased nutrient loading has been linked to the degradation of coral reefs in regions where they are in close proximity to urbanisation, agriculture or industry (Brown 1997, Fabricius 2005, Wooldridge 2009, Fabricius 2011). For example, the development of the catchment surrounding the Great Barrier Reef has resulted in a 5.7 and 8.9 fold increase in the levels of nitrogen and phosphorus, respectively, since European settlement (Kroon et al. 2012). This eutrophication has been quantitatively linked to declines in coral species richness (DeVantier et al. 2006), outbreaks of the corallivorous crown-ofthorns starfish (Acanthaster planci) (Fabricius et al. 2010, Brodie et al. 2017a), and reduced upper thermal bleaching thresholds of inshore coral reefs (Wooldridge 2009, Wooldridge et al. 2017). 
Coral reefs are underpinned by the symbiosis between dinoflagellate algae from the family Symbiodiniaceae and cnidarian stony corals. These symbiotic algae reside within the gastrovascular cavity of their cnidarian host, providing the coral with photosynthates that support up to $100 \%$ of its metabolic requirements (Muscatine et al. 1981, Grottoli et al. 2006, Tremblay et al. 2012), facilitating coral growth and reproduction (Muscatine et al. 1984, Davies 1991). In return, the dinoflagellate algae receive protection, a stable place in the water column and coral waste products such as ammonium (Venn et al. 2008, Yellowlees et al. 2008). Healthy corals contain millions of symbionts per square centimetre of tissue. However, when an environmental perturbation occurs, symbionts themselves can die due to stress and/or they are expelled from coral tissue, causing the coral to lose its pigmentation (see Figure 1.3) (Douglas 2003, Weis 2008). This phenomenon is called 'coral bleaching'. Bleached corals suffer nutrient deprivation, increased susceptibility to disease, and mortality if a symbiosis cannot be re-established (Goreau and Macfarlane 1990, McClanahan 2004). The most prominent causes of bleaching are elevated temperatures and irradiation. It is widely accepted that the global increase in coral bleaching over the last century is a consequence of elevated sea surface temperatures due to global warming (Hughes et al. 2003, Hughes et al. 2017). In conjunction, the importance of nutrient environment in determining the susceptibility and resilience of corals to thermally-induced bleaching is becoming increasingly apparent (Fabricius 2005, Brodie et al. 2012, D'Angelo and Wiedenmann 2014). The influence of eutrophication on the cnidarian-algal symbiosis begins with the effects of eutrophication on the physiology of Symbiodiniaceae, which is the focus of this study.

\subsubsection{The effect of eutrophication on Symbiodiniaceae}

\section{Nutrient assimilation by Symbiodiniaceae}

Nitrogen and phosphorus compounds are necessary for the physiological functioning of plants. Phosphorus is a key component of nucleotides and membrane phospholipids (Lehninger et al. 1993), and nitrogen is an essential component of amino acids, which are the building blocks of proteins (Bothe et al. 2006). Seawater contains both ammonium and nitrate as sources of dissolved inorganic nitrogen (DIN). Ammonium is taken up Symbiodiniaceae by glutamine synthetase (GS) and glutamine 2-oxoglutare aminotransferase (GOGAT) enzymes (Swanson and Hoegh-Guldberg 1998, Roberts et al. 1999, Roberts et al. 2001, Pernice et al. 2012). Nitrate on the other hand is assimilated by an enzymatic cycle involving nitrate and nitrite reductase enzymes and reduced ferredoxins (Fd), from the electron transport chain of photosystem II (Tanaka et al. 2006, Kopp et al. 2015). Comparatively, phosphorus can be sourced in either its organic or inorganic forms (Björkman and 
Karl 1994). Dissolved organic phosphorus (DOP) and particulate organic phosphorus (POP) must be converted into dissolved inorganic phosphorus (DIP) in order to be assimilated (Ferrier-Pagès et al. 2016), a process mediated by alkaline phosphatase activity (APA) (Doonan and Jensen 1980, Vincent and Crowder 1995, Wiedenmann et al. 2013). In contrast to nitrogen, there has been less research on phosphorus assimilation in the cnidarian-algal symbiosis and the exact enzymes involved in this process have not been identified. However, evidence suggests that DIP is absorbed and concentrated from surrounding seawater by carrier-mediated active transport (Jackson and Yellowlees 1990, Godinot et al. 2009, Ferrier-Pagès et al. 2016).

\section{The effect of eutrophication on Symbiodiniaceae physiology}

There have been numerous studies whereby nitrogen or phosphorus levels, or both, have been experimentally or naturally elevated, and various physiological parameters of Symbiodiniaceae in hopsite and/or in culture measured (Muscatine et al. 1989a, Snidvongs and Kinzie 1994, Marubini and Davies 1996, Ferrier-Pagès et al. 2001, Koop et al. 2001, Holcomb et al. 2010, Béraud et al. 2013, Fabricius et al. 2013, Wiedenmann et al. 2013, Ezzat et al. 2015, Rosset et al. 2015, Ezzat et al. 2016b, Rosset et al. 2017). A clear trend in the literature is the importance of the relative abundance of nitrogen and phosphorus, which determines the physiological response of Symbiodiniaceae to elevated nutrient levels (Wiedenmann et al. 2013, D'Angelo and Wiedenmann 2014).

Phosphate enrichment alone enhances the physiological functioning of Symbiodiniaceae and has been shown to increase both photosynthetic and respiration rates, along with the maximum quantum yield of photosystem II (Kinsey and Davies 1979, Ferrier-Pagès et al. 2000, Godinot et al. 2011a). In fact, phosphate enrichment has been shown to reduce the bleaching susceptibility of corals and enhance the translocation and retention of carbon within the host tissue under thermal stress (Ezzat et al. 2016a). In contrast, nitrogen enrichment can have a varied effect on Symbiodiniaceae physiology, driven by the ratio of nitrogen to phosphorus. Under nitrogen-enriched conditions, symbiont growth rates increase (Muscatine et al. 1989b, Stambler et al. 1991, Snidvongs and Kinzie 1994, Marubini and Davies 1996, Wiedenmann et al. 2013, Ezzat et al. 2015, Rosset et al. 2015). This is significant, as elevated Symbiodiniaceae densities also result in an increased demand for carbon and phosphate, which if not met induces physiological dysfunction. More specifically, phosphate starvation of Symbiodiniaceae due to a severely imbalanced nitrogen to phosphorus ratio has been shown to induce a shift in the lipid composition of the thylakoid membrane from phospholipids to sulpholipids, which is thought to alter the ionic character of the membrane and reduce the threshold for thermal and light- 
induced damage (Wiedenmann et al. 2013). This physiological response is likely linked to the reduced photosynthetic efficiency and light-harvesting ability of Symbiodiniaceae observed under elevated levels of DIN (Nordemar et al. 2003, Béraud et al. 2013, Wiedenmann et al. 2013, Rosset et al. 2017). Notably, recent studies directly comparing nitrogen enrichment alone with both nitrogen and phosphorus enrichment, have found that symbiont photosynthetic dysfunction, thermally-induced bleaching, and a reduced translocation of photosynthate by symbionts, is greater under a severely imbalanced nutrient regime in favour of nitrogen (Wiedenmann et al. 2013, D'Angelo and Wiedenmann 2014, Ezzat et al. 2015, Ezzat et al. 2016a, Rosset et al. 2017). While the physiological responses of Symbiodiniaceae to elevated nutrients and temperature are well characterised, the cellular mechanisms which underlie these responses are not well understood.

\subsection{4 'Omics' techniques and their application in increasing our understanding of the cnidarian-dinoflagellate symbiosis}

In the face of global coral reef decline, it is essential to understand the mechanism of coral bleaching. However, while the environmental stressors that trigger bleaching are well established, the cellular and molecular pathways that result in symbiont loss are not well understood (Weis 2008, Davy et al. 2012). Partially, this is because of the difficulty of elucidating physiological responses on a molecular level. However, this is becoming increasingly possible with the progressive development of 'omics' techniques such as genomics, transcriptomics, proteomics and metabolomics (Meyer and Weis 2012). These omics techniques have already dramatically enhanced our knowledge of the cnidariandinoflagellate symbiosis and the bleaching response. For example, genomics has shed light on the response of corals to environmental change on a molecular level (Shinzato et al. 2011), and metabolomics studies have provided insight into the cellular response to thermal stress in both the cnidarian host and symbiont (Hillyer et al. 2016b, Hillyer et al. 2017).

In general, genomics studies of Symbiodiniaceae are lagging behind those of their cnidarian hosts (Meyer and Weis 2012), which has had a knock-on effect to both transcriptomics and proteomics. This is partially because of the huge genome of dinoflagellates (LaJeunesse et al. 2005, Hou and Lin 2009, Leggat et al. 2011), making sequencing difficult. In conjunction, the Symbiodiniaceae family is incredibly diverse, and still not fully characterised. However, as sequencing technology improves, Symbiodiniaceae genomes are becoming increasingly available, which has facilitated a recent jump in progress, particularly with regards to transcriptomic studies on Symbiodiniaceae. There have been a series of transcriptomic studies published recently on Symbiodiniaceae, for example investigating the 
response of the transcriptome to thermal stress (Kaniewska et al. 2015, Gierz et al. 2017). However, it is worth noting that protein abundance is largely regulated by post-translational modification in

dinoflagellates generally, and Symbiodiniaceae specifically (Okamoto et al. 2001, Chen et al. 2004, Chen et al. 2005, Bachvaroff and Place 2008, Moustafa et al. 2010, Ganot et al. 2011, Leggat et al. 2011). Thus, proteomics may provide further insight into the cellular response of Symbiodiniaceae to environmental stressors.

Initially proteomics relied on the separation of protein using gel electrophoresis, from which protein bands were excised and analysed with mass spectrometry for identification. However, this method is limited and can merely identify tens of proteins in a sample (Stochaj and Grossman 1997, Pasaribu et al. 2014, Pasaribu et al. 2015). Since then, this field has grown immensely as mass spectrometry technology has become increasingly sensitive and able to identify proteins from more complex mixtures (Tyers and Mann 2003). Now using LC-ESI-MS/MS (liquid chromatography- electro-spray ionisation- tandem mass spectrometry), hundreds of proteins can be both quantified and identified using genome-specific protein databases. This technology has been applied to the cnidarian host, allowing the investigation of differential protein expression between symbiotic and aposymbiotic Exaiptasia pallida ('Aiptasia'), a model cnidarian, to provide insight into the coral-dinoflagellate symbiosis (Oakley et al. 2016). However, the latest in proteomics technology is yet to be applied to Symbiodiniaceae. Thus, the utilisation of LC-ESI-MS/MS to identify proteins extracted from Symbiondiniaceae in this thesis is novel, providing detail of the cellular response to environmental stress that is currently lacking in this field of research.

\subsubsection{Aims of this study}

Eutrophication of coastal ecosystems can destabilise the cnidarian-dinoflagellate symbiosis, which underlies the success of coral reefs, and the productivity and biodiversity which they support (Odum and Odum 1955, Reaka-Kudla et al. 1997). Clearly, in combination with the threats that coral reefs face due to global warming, it is important to gather knowledge on the effects of eutrophication on both corals and their endosymbionts, as effective management of this local stressor may help build the resilience of these organisms to future temperature extremes (D'Angelo and Wiedenmann 2014). With regards to coral bleaching, recent literature has highlighted that the effect of nutrient enrichment on the coral holobiont begins with the reduction of Symbiodiniaceae stress tolerance, paradoxically via imbalanced nutrient ratios resulting in deprivation of nutrients essential to the physiological functioning of the symbiont (Wiedenmann et al. 2013, D'Angelo and Wiedenmann 2014, Rosset et al. 
2017). While the physiological effects of eutrophication and its interaction with thermal stress have been established, the molecular mechanisms behind these responses are not fully characterised.

This study addressed this knowledge gap by aiming to determine the influence of nutrient supply on the proteomic response to elevated temperature of $B$. minutum, and so characterise the detailed cellular responses of this symbiotic dinoflagellate. This objective is achieved by utilising powerful proteomics techniques (LC-ESI-MS/MS), to compare the proteome of algae under different nutrient regimes at control $\left(25^{\circ} \mathrm{C}\right)$ and elevated temperatures $\left(34^{\circ} \mathrm{C}\right)$. In addition, various physiological measurements were taken to corroborate trends of protein expression. The measurements performed were: symbiont density, pulse amplitude modulation (PAM) fluorometry, chlorophyll content, photosynthetic and respiratory oxygen flux, and alkaline phosphatase activity. In terms of the physiological effects of elevated temperature, it was hypothesised that those cultures exposed to an imbalanced nutrient regime would show greater susceptibility to thermal stress than cultures under a balanced regime, as observed in other studies (Wiedenmann et al. 2013, D'Angelo and Wiedenmann 2014, Ezzat et al. 2015, Ezzat et al. 2016a, Rosset et al. 2017). In conjunction, it was predicted that protein expression would change in response to both temperature and nutrient regime, and that there would be an interaction between these two environmental factors on the Symbiodiniaceae proteome. Specifically, it was predicted that nutrient regime would influence the abundance of proteins involved in metabolism, such as those involved in glucose metabolism, and energy generation pathways like glycolysis and the tricarboxylic acid (TCA) cycle, as has been observed in transcriptomic and proteomic studies of higher plants (Li et al. 2007, Müller et al. 2007, Li et al. 2008, Zhang et al. 2014) and diatoms (Brembu et al. 2017) under different nutrient regimes. It was also thought that temperature would affect the expression of metabolic proteins, with higher temperatures resulting in an upregulation of proteins involved in energy-generating pathways. Specifically, it was predicted that the TCA cycle and glycolysis would increase, while pathways involved in energetically-costly cellular processes such as biogenesis would decrease, as has been inferred previously from metabolomic studies of Symbiodiniaceae (Hillyer et al. 2016a, Hillyer et al. 2017). Furthermore, it was hypothesised that temperature would affect the abundance of proteins that function to maintain cellular homeostasis under thermal stress, such as antioxidants and chaperonin proteins (Krueger et al. 2014, Gierz et al. 2017). 


\subsection{Materials and Methods}

\subsubsection{Experimental organism}

The Symbiodiniaceae species used here was Breviolum minutum (ITS2 'type' B1), the homologous symbiont of the model cnidarian Aiptasia. This dinoflagellate is considered moderately robust to thermal and oxidative stress (Wietheger et al. 2015), and thus occupies an intermediate position on the spectrum of species-specific thermal tolerance (Swain et al. 2017). Unfortunately, the NZ01 strain of Breviolum minutum used in chapter 2 was no longer available, thus only the FlAp2 strain was used for the following experiment.

\subsubsection{Validation of nutrient treatments}

The nutrient treatments were maintained at ambient (control) (DIN $\sim 1.8 \mu \mathrm{M}$, DIP $\sim 0.2 \mu \mathrm{M}$ ), imbalanced (DIN $\sim 26 \mu \mathrm{M}$, DIP $\sim 0.5 \mu \mathrm{M}$ ), or enriched (DIN $\sim 3 \mu \mathrm{M}$, DIP $\sim 0.6 \mu \mathrm{M}$ ) conditions. All nutrient regimes used for this experiment were chosen to fall within the range of values observed on natural coral reefs worldwide. DIN typically ranges from 0.05-9.8 $\mu \mathrm{M}$ (O'Neil and Capone 2008), but can be as high as $28.1 \mu \mathrm{M}$ in polluted waters (Lapointe 1997). DIP ranges from 0.08 to $0.3 \mu \mathrm{M}$ (Szmant 2002) but can get up to $1.42 \mu \mathrm{M}$ in areas exposed to severe terrestrial run-off (Da Silva Costa 2001). Culture medium was prepared with Red Sea pro-salt artificial seawater and double distilled water, with no addition of nutrients for the ambient nutrient level treatment, and the addition of sodium nitrate and di-sodium hydrogen phosphate for the enriched and imbalanced media. The exact nutrient levels were tested in both the stock solution of the medium prior to the experiment and directly from the medium in the glass tubes containing $B$. minutum at the end of the experiment. Analysis was performed by NIWA Water Quality Laboratory (Hamilton, New Zealand).

At the end of the experimental period, nitrogen concentration was slightly reduced in all cases, reflecting utilisation by the Symbiodiniaceae (Table 3.1). In comparison, there was a slight elevation in phosphorus levels, perhaps resulting from cellular leakage when water samples were frozen before being filtered and analysed. In support of this, the difference between the expected and measured phosphorus levels was similar across treatments. In spite of this discordance, nutrient ratios still remained within the intended range and the treatment was maintained. As water was refreshed by half the total volume daily it is assumed that, at any one time, the nutrient environment of the various treatments was between the concentration in the stock solutions and the value measured at the end of the experimental period. 
Table 3.1: Nitrogen and phosphorus levels of the ambient, enriched and imbalanced nutrient treatments at the start and end of the experiment $(\mu \mathrm{M})$.

\begin{tabular}{|c|c|c|c|c|c|c|}
\hline & \multicolumn{3}{|c|}{ Stock nutrient medium } & \multicolumn{3}{|c|}{$\begin{array}{l}\text { Medium from algal cultures at the end of the } \\
\text { experimental period }\end{array}$} \\
\hline $\begin{array}{l}\text { Nutrient } \\
\text { Regime }\end{array}$ & Total Nitrogen & $\begin{array}{c}\text { Total } \\
\text { phosphorus }\end{array}$ & $\begin{array}{c}\text { Ratio of } \\
\text { Nitrogen to } \\
\text { phosphorus }\end{array}$ & Total Nitrogen & $\begin{array}{l}\text { Total } \\
\text { phosphorus }\end{array}$ & $\begin{array}{c}\text { Ratio of } \\
\text { Nitrogen to } \\
\text { phosphorus }\end{array}$ \\
\hline $\begin{array}{l}\text { Ambient: Low } \\
\text { nitrogen, low } \\
\text { phosphorus }\end{array}$ & 2.6 & 0.1 & 27 & 1.0 & 0.3 & 4 \\
\hline $\begin{array}{c}\text { Enriched: } \\
\text { High } \\
\text { nitrogen, } \\
\text { high } \\
\text { phosphorus }\end{array}$ & 4.6 & 0.5 & 9 & 1.3 & 0.6 & 2 \\
\hline $\begin{array}{l}\text { Imbalanced: } \\
\text { High } \\
\text { nitrogen, low } \\
\text { phosphorus }\end{array}$ & 26.5 & 0.4 & 75 & 26 & 0.6 & 42 \\
\hline
\end{tabular}

\subsubsection{Experimental design}

Symbiodiniaceae cultures under an ambient, imbalanced or enriched nutrient regime, were either kept at control temperatures $\left(25^{\circ} \mathrm{C}\right)$ or exposed to a temperature increase from $25^{\circ} \mathrm{C}$ to $34^{\circ} \mathrm{C}$ over a oneweek period, then maintained at $34^{\circ} \mathrm{C}$ for 48 hours (Figure 3.1 A). Bleaching thresholds depend on geographic location but generally range between 27 to $36.8^{\circ} \mathrm{C}$ (Hoegh-Guldberg 1999, Fitt and Cook 2001, Coles and Brown 2003, Hoegh-Guldberg et al. 2004, Jokiel 2004). Thus, an upper-temperature maximum of $34^{\circ} \mathrm{C}$ was chosen for this experiment, to ensure a physiological response was observed while retaining ecological relevance. There were 32 biological replicates per nutrient regime; 16 kept at control temperature and 16 exposed to elevated temperature. 
A)

Temperature controlled water baths
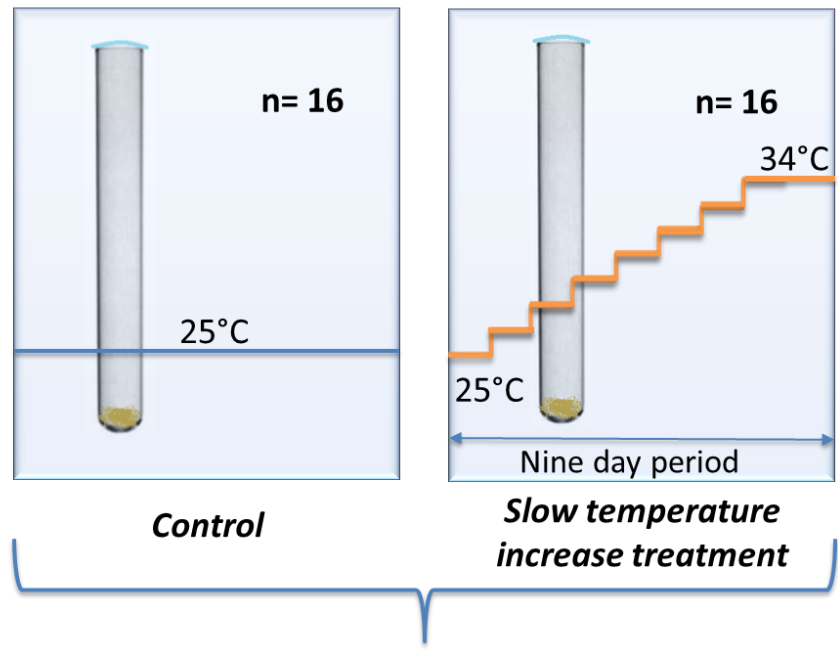

Under three different nutrient regimes:

- Ambient, Imbalanced, Enriched

- $\mathrm{n}=16$ biological replicates per treatment
B)

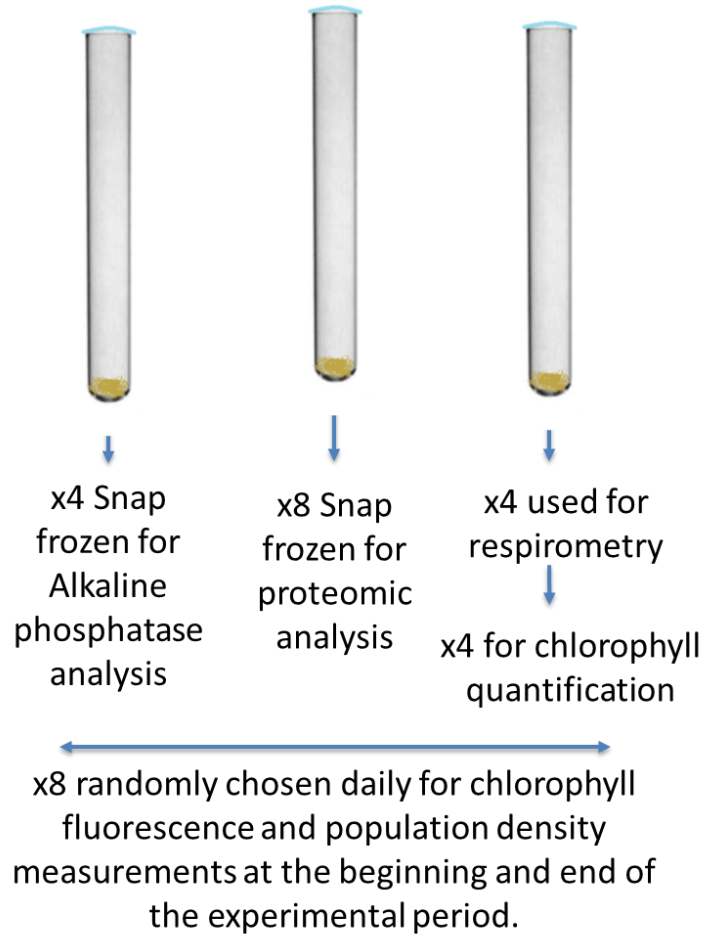

Figure 3.1: A) Visualisation of experimental design. B) Allocation of biological replicates to the various physiological measurements performed in this study.

\subsubsection{Experimental procedure}

\section{Set up of the cultures for the experiment}

The $B$. minutum cultures had previously been maintained in $\mathrm{f} / 2$ medium at $25^{\circ} \mathrm{C}$, on a $12: 12 \mathrm{~h}$ light dark cycle in a temperature-controlled incubator $\left(\mathrm{PAR}=40 \mu \mathrm{mol}\right.$ photons $\mathrm{m}^{-2} \mathrm{~s}^{-1}$, warm fluorescent lamps). Prior to the experiment, $20 \mathrm{ml} \mathrm{B.} \mathrm{minutum} \mathrm{stock} \mathrm{culture} \mathrm{was} \mathrm{centrifuged} \mathrm{at} \mathrm{20,000} \times \mathrm{g}$ for 6 min. The supernatant was removed and the remaining algal pellet resuspended in $10 \mathrm{ml}$ of treatment medium containing either ambient, imbalanced or enriched nutrient levels, and vortexed. This process was repeated twice more to wash the algal cells thoroughly of the original $f / 2$ medium. The final $B$. minutum suspension was then transferred to a $250 \mathrm{ml}$ glass conical flask and acclimated for twoweeks to the experimental nutrient conditions.

After this acclimation period, B. minutum cells were aliquoted into $10 \mathrm{ml}$-glass culture tubes. The stock cell density was first measured with a haemocytometer and adjusted, so that approximately 5 million cells in a volume of $2 \mathrm{ml}$ was added to each tube. The culture tubes were then placed in a 
water bath on 12:12 h light dark cycle (PAR $=60-65 \mu \mathrm{mol}$ photons $\mathrm{m}^{-2} \mathrm{~s}^{-1}$ (T12 florescent light bulbs, warm light), and allowed to acclimate to the increased light intensity for one week before the experiment began. $4 \mathrm{ml}$ of the treatment medium (i.e. half of the total volume) was exchanged daily, to ensure a relatively consistent nutrient regime. Note that, given that the respirometry measurements could not be physically completed in one day, the control and elevated temperature treatment were staggered by $24 \mathrm{~h}$.

\section{Sample processing}

At the end of the experiment, all cultures were transferred to $15 \mathrm{ml}$ centrifuge tubes and centrifuged for $8 \mathrm{~min}$ at $2000 \times \mathrm{g}$. The medium was then decanted and the algal pellet resuspended in $0.5 \mathrm{ml}$ of the appropriate treatment medium and transferred to a $2 \mathrm{ml}$ tube. A further $0.7 \mathrm{ml}$ treatment medium was used to rinse the $15 \mathrm{ml}$ centrifuge tube and added to the previous $0.5 \mathrm{ml}$, bringing the final volume to $1.2 \mathrm{ml}$. A $200 \mu \mathrm{l}$ aliquot of this suspension was taken from each tube for cell counts. Four tubes per temperature treatment were set aside for respirometry measurements and the remaining tubes were spun down for $3 \mathrm{~min}$ at $2000 \times \mathrm{g}$ and the supernatant decanted. The resulting algal pellets were then snap frozen at $-80{ }^{\circ} \mathrm{C}$ for subsequent analysis (Figure 3.1).

\section{Physiological measurements}

Culture population growth, chlorophyll fluorescence, chlorophyll concentration, photosynthetic and respiratory oxygen flux, and alkaline phosphatase activity were measured as described in Chapter 2, section 2.2.4.

\subsubsection{Statistical tests for physiological measurements}

GraphPad Prism v. 7.04 in conjunction with IBM SPSS Statistics $v 25$ was used to perform statistical tests and create graphs for physiological data.

Unpaired two-tailed t-tests, not assuming constant variance, were used to compare quantum yield measurements of control and treatment cultures on a daily basis $(\alpha=0.01)$. In some cases, the unpaired t-test identified significant differences between the quantum yield measurements of control and treatment cultures at the beginning of the experimental period, when they were both kept at the 
same temperature. These discrepancies are likely due to biological variability between replicates and are not discussed in the results section as they do not contribute to the overall trends. For the quantum yield measurements of cultures under the imbalanced nutrient regime a repeated measures ANOVA was performed in placed of multiple unpaired T-tests, due to a consistent difference between control and treatment cultures throughout the experimental period. To determine how the control and treatment measurements changed over time each measurement from day 16 onwards was compared with the average fluorescent measurement over days 9-15 of the experiment when all cultures including treatments were kept at $25^{\circ} \mathrm{C}$ (control conditions) $(\alpha=0.01)$.

For all other physiological measurements, a two-way ANOVA was used to compare the main effects of temperature and nutrient regime, followed by a Tukey post hoc analysis $(\alpha=0.05)$. If there was an interaction between these two variables, the ANOVA was re-run with simple effects, followed by a Dunnet's multiple comparisons post hoc test $(\alpha=0.05)$. ANOVA assumptions of normality and equal variance were assessed using the Shapiro-Wilk and Kolmogornov-Smirnov normality tests, and the Levene's and equal variance test. If these assumptions were not met even after data transformation, a Kruskal-Wallis test followed by a Dunnets multiple comparison test was performed $(\alpha=0.05)$. In addition, the effect of temperature within nutrient regime was also tested using an unpaired two-tailed t-test, or a Mann Whitney U-test if assumptions of normality weren't met $(\alpha=0.05)$. All p-values are Bonferroni-adjusted to avoid Type 1 errors. Data points that were less or more than two standard deviations of the average were removed as outliers. Details of statistical test are in appendix section A2.1.

\subsubsection{Proteomics methodology}

\section{Protein extraction}

This protocol was adapted from Wiśniewski et al. (2009). All protein handling was done in low protein binding tubes to avoid protein loss.

The frozen algal samples were washed twice with High Performance Liquid Chromatography (HPLC) grade water to remove salt. The pellet was then suspended in 5\% sodium deoxycholate (SDC) buffer and ultrasonicated for 20 pulses to lyse cells. B-mercaptoethanol (BME) was then added to $1 \%$ and the sample incubated at $85{ }^{\circ} \mathrm{C}$ for 20 min to denature proteins. Two volumes of ethyl acetate were added and the sample vortexed for $1 \mathrm{~min}$, then centrifuged for $1 \mathrm{~min}$ at $10,000 \times \mathrm{g}$ to separate aqueous and organic layers. The upper ethyl acetate layer was then discarded. This ethyl acetate wash was 
repeated three times to remove photosynthetic pigments, then the lower aqueous layer (sample) was transferred to a molecular weight cut off filter, leaving pellet debris behind. The sample was then centrifuged through the spin filter for $15 \mathrm{~min}$ at $14,000 \times \mathrm{g}$ and the flow-through was discarded. The sample in the filter was then resuspend in $380 \mu \mathrm{l}$ of $50 \mathrm{mM}$ Tris and centrifuged for another $15 \mathrm{~min}$ at $14,000 \times \mathrm{g}$. This wash was repeated once more, and a $10 \mu \mathrm{l}$ sub-sample was taken, diluted 10-fold, acidified and centrifuged to remove any remaining SDC, and assayed for fluorescence-based protein quantification with a Qubit® Protein Assay Kit.

Based on this measurement, $1 \mu \mathrm{g}$ of trypsin was added to the sample per $100 \mu \mathrm{g}$ of total protein and the sample incubated overnight at $37^{\circ} \mathrm{C}$ for digestion. The sample was then centrifuged through the spin filter into a new collection tube, separating the peptides from the undigested protein. Formic acid was then added to $1 \%$ final volume to terminate trypsin digestion. The sample was then vortexed briefly, centrifuged for $1 \mathrm{~min}$ at $16,000 \times \mathrm{g}$ and transferred to a new tube. The peptides were then desalted with C18 tips (Agilent Technologies Bond Elute), dried completely, and resuspended in 70 $\mu 10.1 \%$ formic acid.

\section{Identification of proteins using LC-ESI-MS/MS and MaxQuant proteomics software}

Samples were analysed by liquid chromatography-electrospray ionisation-tandem mass spectrometry (LC-ESI-MS/MS) using methods similar to those of Oakley et al. 2017, with a nonlinear $300 \mathrm{~min}$ gradient (buffer A: 0.1\% formic acid; buffer B: $80 \%$ acetonitrile, $0.1 \%$ formic acid) at $0.3 \mu \mathrm{L} \mathrm{min}^{-1}$ on an Acclaim PepMap C18, $3 \mu \mathrm{m}, 100$ Å column (Thermo Scientific, Auckland, New Zealand) and Ultimate 3000 liquid chromatograph (Dionex, Sunnyvale, CA). Peptides were analysed with an LTQ Orbitral XL (Thermo Scientific) by injection at a $2.2 \mathrm{kV}$ spray voltage and a resolution of 30,000. The top six MS peaks were analysed by the ion trap, rejecting +1 charge states with dynamic exclusion enabled (180 s) (Oakley et al. 2017). The instruments were operated with Chromeleon Xpress (v2.11.0.2914, Dionex), Thermo Xcalibur (v2.1), and ThermoTune Plus (v2.5.5, Thermo Scientific). Each sample was analysed twice as technical replicates.

Protein identification and quantification was conducted using the Andromeda search engine in MaxQuant against the Symbiodinium microadriaticum trEMBL database (Uniprot), plus common contaminants (Cox and Mann 2008). False discovery rate (FDR) thresholds were set at 0.01 for peptide and protein search matches, and a minimum of two peptides per protein was required for identification. Searches assumed trypsin digestion with a maximum of two missed cleavages. 
Oxidation of methionine and acetylation of the protein N-terminus were specified as variable modifications, and carbamidomethylation of cysteine was specified as a fixed modification. Proteins were quantified by label free quantification, with the match between runs feature used to increase the quality of protein quantification between technical replicates (Cox et al. 2014).

\section{Bioinformatic analysis}

R software (v 3.4.3) and Perseus software (v 1.5.0.15) was used for statistical analysis (http://www.perseus-framework.org, (Tyanova et al. 2016). For each biological replicate, protein LFQ intensities and S. microadriaticum sequence matches were imported into Perseus software to create a matrix. Proteins were filtered for contaminants and reverse (false) identifications. All values were normalised by $\log _{2}$ transformation. Proteins that were not identified in at least three biological replicates were then removed. Missing values were imputed from a normal distribution.

With R software (v 3.4.3) a permutational multivariate analysis of variance (PERMANOVA) was performed on the matrix produced by Perseus (results in appendix A2.2). This analysis was used to identify if there was a significant effect of nutrients and temperature on the proteomic data set overall and determine if there was an interaction between these two factors.

A principal component analysis (PCA) was performed with Perseus to visualise the spread of the data and the effect of temperature and nutrient availability on the B. minutum proteome. Also using Perseus differentially abundant proteins between nutrient and temperature treatments were calculated with a student's t-test. Significance was determined with both a Benjamini-Hochberg corrected pvalue $(p<0.05)$ (presented in tables S1, S2 and S3 in the appendix), and the more conservative permutation-based FDR procedure, which adjusts for multiple testing. 1000 permutations were performed with the FDR set to two thresholds, 0.05 and 0.1 . These differences are visualised with Hawaii plots in Figures 3.8 and 3.9.

\section{Protein functional annotation}

The protein sequence matches of differentially abundant proteins between treatments were annotated against the Uniprot SwissProt database using Diamond BLAST, taking the match with the lowest Evalue (Buchfink et al. 2015), in order to match these sequences with Uniprot KB accessions. Diamond BLAST was run in "more sensitive mode" with a maximum E-value of $1 \times 10^{-3}$. These accessions 
were then individually searched in the Uniprot KB database and the available gene annotation information was used to organise proteins into gene ontology (GO) biological functions and processes. The organised tables of all classified differentially abundant proteins were too large to include in the results section and so are located in appendix section A2.2. Notably, if a functional group only contained one protein it was not included in these tables unless it was of particular interest. To represent this data in the results section, bar graphs were produced of protein counts in each functional group, with a minimum of three proteins per group (Figures 3.8, $3.10 \& 3.12$ ).

\section{Protein interaction analysis}

Proteins identified as being differentially abundant between treatments were also analysed with the STRING database (Szklarczyk et al. 2018). This software produces a protein-protein interaction network based on known and predicted protein functional associations (i.e. both involved in a specific biological function) (combined score > 0.5) (Szklarczyk et al. 2018). The functional associations are determined by co-expression, text-mining, biochemical and genetic experimental data and previously annotated pathway and protein-complex knowledge (see Szklarczyk et al. 2018). This analysis determines whether there are more functional interactions between the set of differentially abundant proteins than expected by chance (i.e. more interactions than what would be expected for a random set of proteins of a similar size, Szklarczyk et al. 2018). In addition, the STRING software performs an enrichment analysis, producing enrichment values for KEGG (Kyoto Encyclopedia of Genes and Genomes) pathways and GO biological processes. A limitation of the STRING database for Symbiodiniaceae research is that a subject organism must be chosen for an interaction network to be produced, and Symbiodiniaceae or dinoflagellates are yet to be included in the database. Thus, Arabidopsis thaliana was chosen as it has the best described plant genome, reducing the number of proteins involved in the analysis as not all Symbiodiniaceae genes are homologous with Arabidopsis.

\subsection{Results}

\subsubsection{Physiological measurements}

\section{Chlorophyll a fluorescence measurements}

Across nutrient regimes, elevated temperature had a negative effect on chlorophyll $a$ fluorescence, with this difference being more noticeable for dark-adapted measurements than light adapted ones. 
After $24 \mathrm{~h}$ at $34^{\circ} \mathrm{C}$, the maximum quantum yield of the cultures under enriched and ambient nutrient regimes was 31 and 33\% below that of the control, respectively (unpaired two-tailed t-test: $\mathrm{p}<0.0001$ for both), indicating down-regulation of photosystem II and thermally-induced photoinhibition. Under the imbalanced nutrient regime, the maximum quantum yield was below that of the control for almost the entire experimental period, and so could not be directly compared to the control (Figure 3.2 D). Thus, a repeated-measures ANOVA was performed, to compare how both the control and treatment measurements changed over time. As the controls were also significantly lower, on average, on day 18 of the experiment, no inferences can be made about the treatment culture maximum quantum yield measurement on this day. The fluorescence measurements of treatment cultures were significantly lower at 31 to $34^{\circ} \mathrm{C}$ (by $10-18 \%$ ), compared to measurements at $25^{\circ} \mathrm{C}$, indicating a moderate downregulation of photosystem II in response to elevated temperature. 
A)

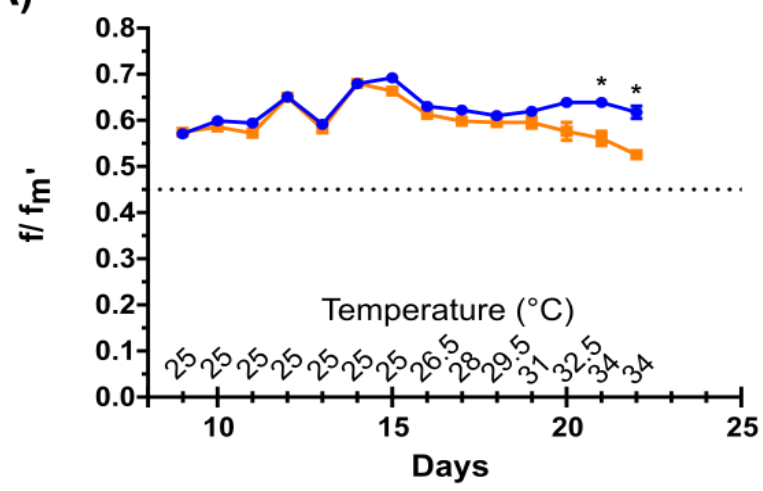

C)

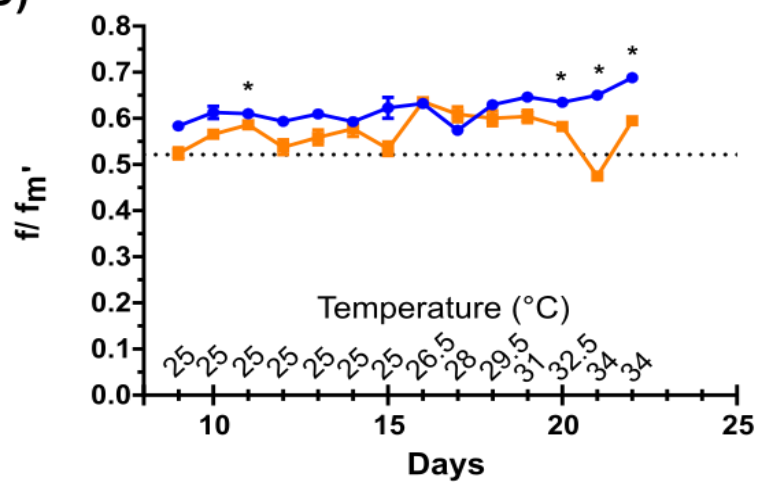

E)

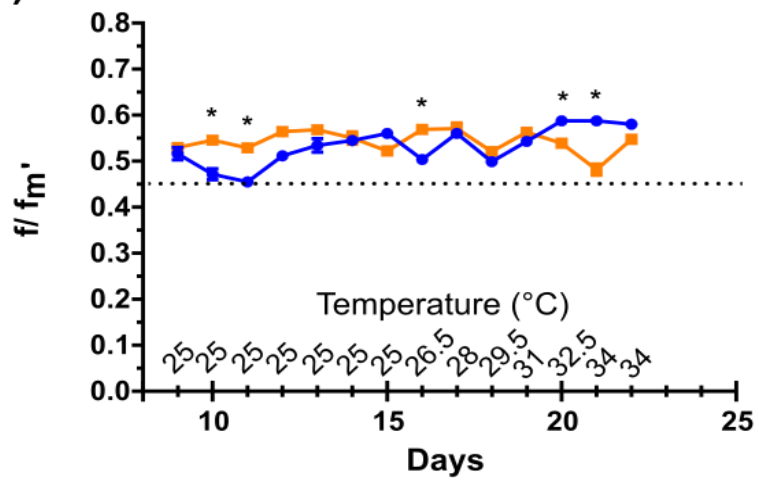

$\rightarrow$ Control
B)

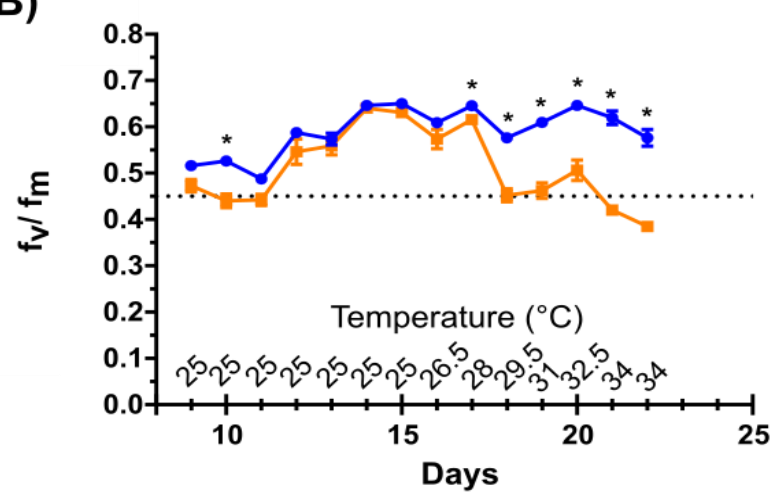

D)

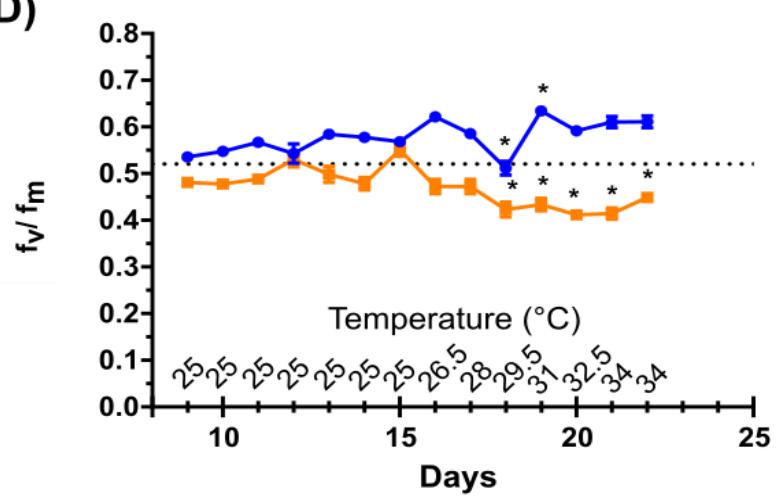

F)

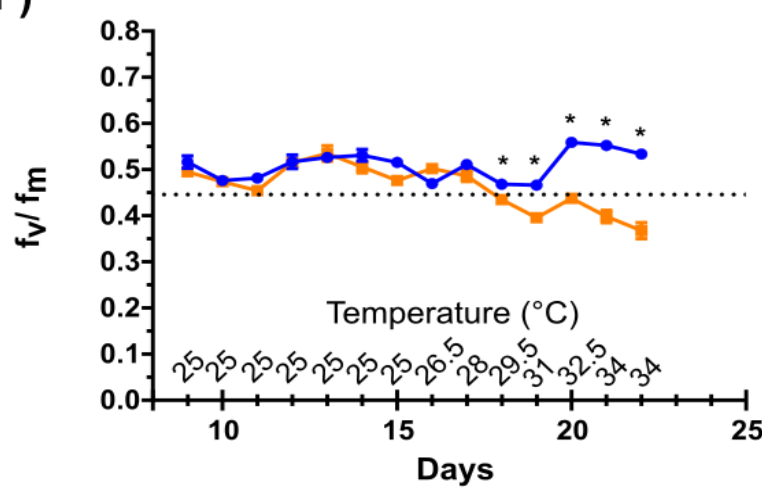

Figure 3.2: Light and dark-adapted chlorophyll $a$ fluorescence measurements of $B$. minutum cultures under ambient, imbalanced and enriched nutrient regimes. A) Effective quantum yield $\left(\mathrm{f}_{\mathrm{v}} / \mathrm{f}_{\mathrm{m}}\right.$ ) for cultures under ambient nutrient conditions. B) Maximum quantum yield $\left(\mathrm{f}_{\mathrm{v}} / \mathrm{f}_{\mathrm{m}}\right)$ for cultures under ambient nutrient conditions. C) Effective quantum yield for cultures under imbalanced nutrient conditions. D) Maximum quantum yield for cultures under imbalanced nutrient conditions. E) Effective quantum yield for cultures under enriched nutrient conditions. F) Maximum quantum yield for cultures under enriched nutrient conditions. Control cultures (blue) were maintained at $25^{\circ} \mathrm{C}$ for the entire experimental period. Treatment cultures (orange) were taken from $25^{\circ} \mathrm{C}$ to $34^{\circ} \mathrm{C}$ over a seven-day period as indicated by the x-axis. Values presented are the mean $+/$ - standard error, if error bars are not seen they are smaller than the data point. The dotted line indicates the value above which yield measurements are considered within the healthy range (Gorbunov et al. 2001). Asterisks indicate statistically significant differences between the control and treatment culture measurements determined by a unpaired two-tailed t-test. Except for graph D, where asterisks indicate whether measurements have changed over time for the control and treatment culture senaratelv ( reneated 


\section{Pigment concentration}

Both nutrient regime and temperature had no significant effect on the cellular concentration of chlorophyll $a$ or carotenoids (Figure 3.3 A \& B). However, for the ratio of carotenoids to chlorophyll $a$, while temperature had no effect, nutrient regime significantly altered the pigment ratio (two-way ANOVA, $\mathrm{f}(2,18)=24.08, \mathrm{p}<0.0001)$. Both imbalanced and enriched nutrient regimes had significantly higher (by about 20\%) ratios of carotenoids to chlorophyll $a$ than did cultures maintained under ambient nutrient levels, regardless of temperature (Tukey post hoc, all p-values $<0.03$; see appendix section A2.1 for details).

\section{Alkaline phosphatase activity}

Both temperature and nutrient regime had a significant influence on APA (Figure 3.3 D). Under the ambient and imbalanced nutrient regimes APA was $36 \%$ greater at $34^{\circ} \mathrm{C}$ relative to $25^{\circ} \mathrm{C}$ (respectively: unpaired t-test, $\mathrm{p}=0.002$, and Mann Whitney U-test, $\mathrm{p}=0.03$ ). Overall, those cultures under the imbalanced nutrient regime had the highest APA (Tukeys multiple comparison test: $\mathrm{p}<$ 0.001for both comparisons).

\section{Population growth rates}

Population growth rates were significantly affected by both nutrient regime and temperature (Figure $3.3 \mathrm{E})$. Population growth was highest under the ambient nutrient regime at $34^{\circ} \mathrm{C}$, and under the enriched nutrient regime (at both 25 and $34^{\circ} \mathrm{C}$ ). Alternately, under ambient nutrient levels at $25^{\circ} \mathrm{C}$, and the imbalanced nutrient regime (at both 25 and $34^{\circ} \mathrm{C}$ ) growth rates were significantly lower (Dunn's multiple comparison test, $\mathrm{p}<0.02$ for all; details in appendix). Temperature had no significant effect on population growth under imbalanced nutrient conditions. However, population growth increased with temperature under both control and enriched nutrient regimes, by 81 and $29 \%$ respectively (unpaired t-test, $\mathrm{p}<0.0001, \mathrm{p}=0.026$ ). 
A)

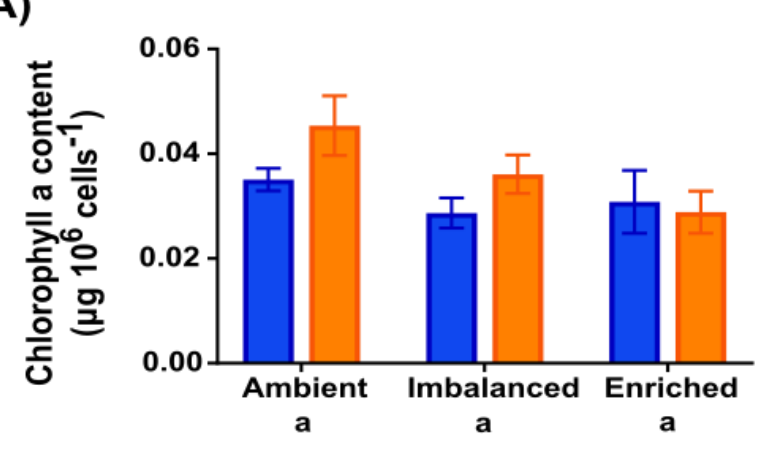

B)
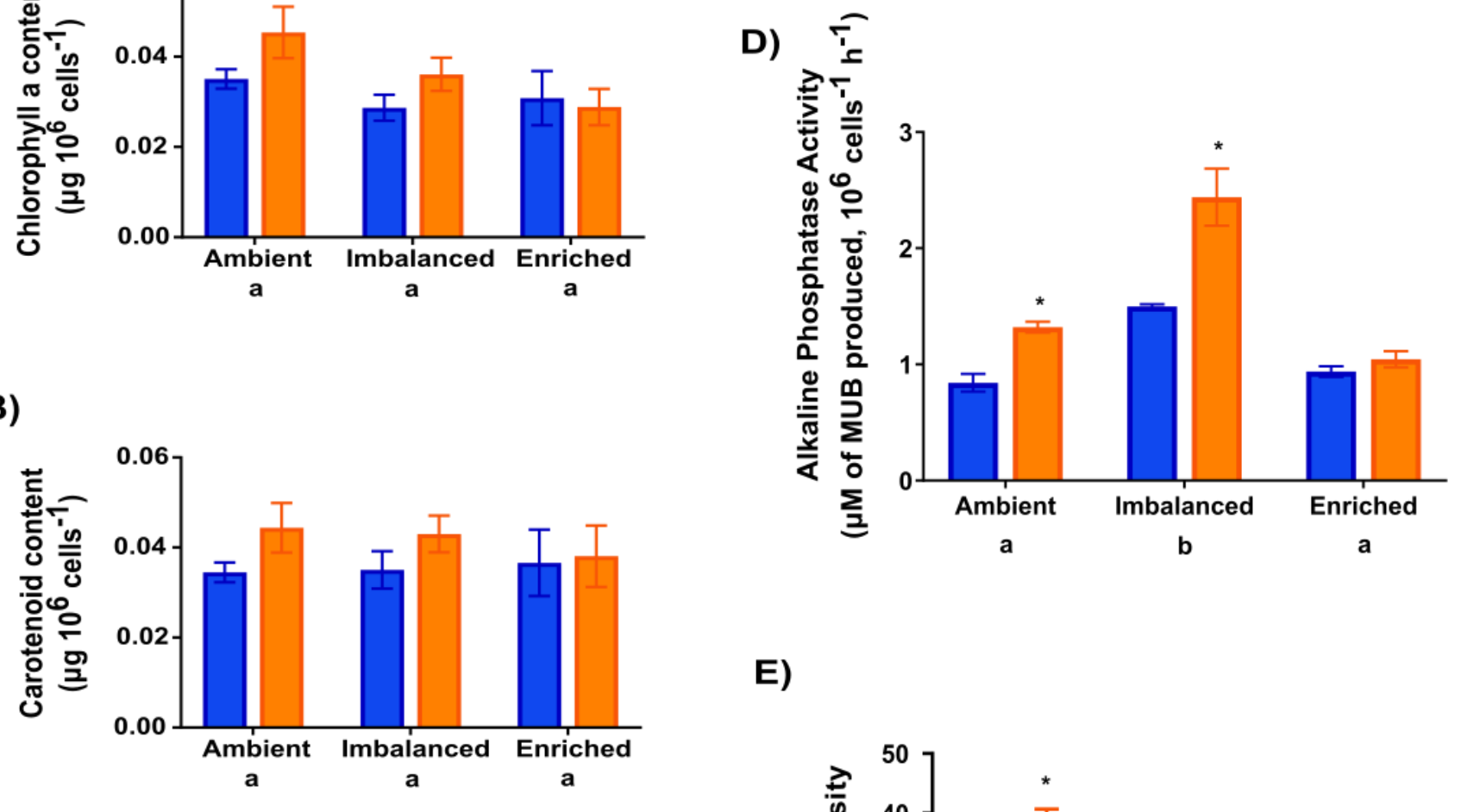

E)
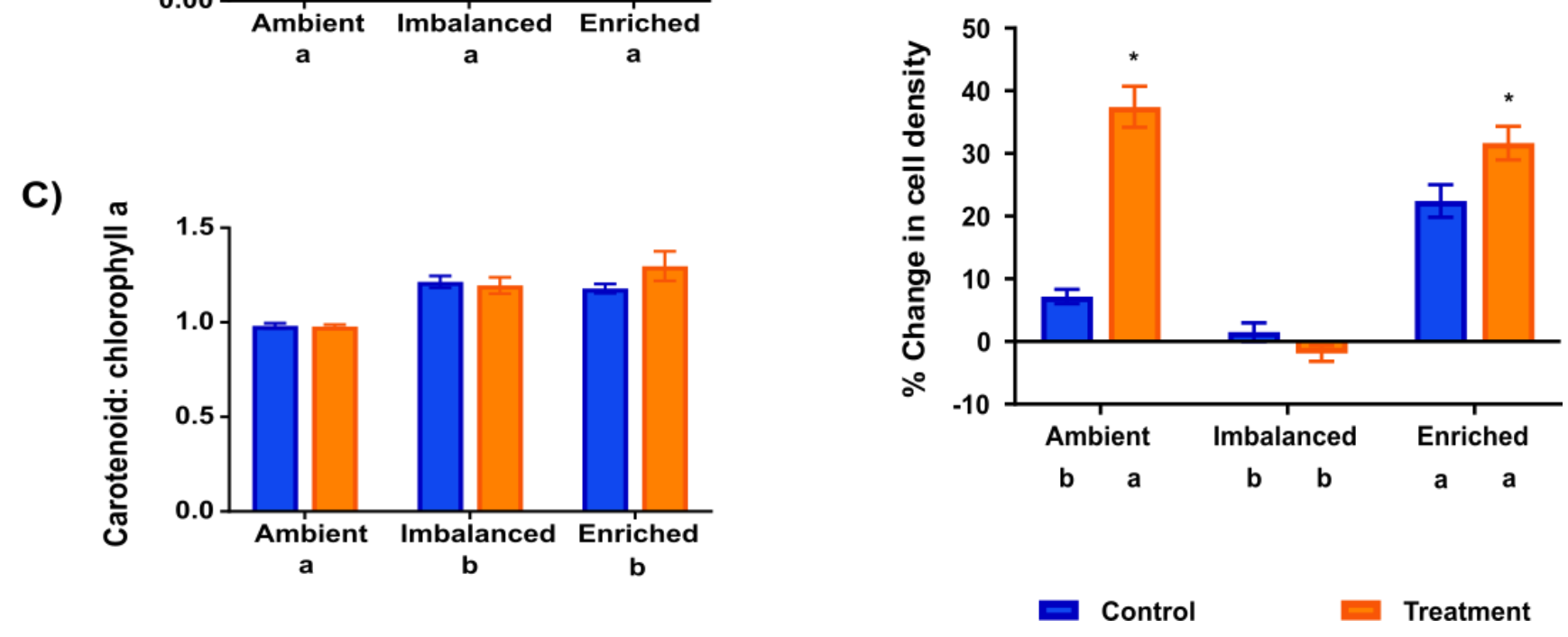

Figure 3.3: (A), (B): The concentration of both chlorophyll a and carotenoids (carotenes and xanthophylls). A) Chlorophyll a concentration; B) Carotenoid concentration (carotenes and xanthophylls); C) The ratio of carotenoids to chlorophyll a ( $\mathrm{n}=4$ for all). (D) Alkaline phosphatase activity ( $\mathrm{n}=4$ for all). (E) The percentage difference in the cell density of cultures from the beginning to the end of the experimental period ( $\mathrm{n}=8$ for ambient, 9 for imbalanced, 10 for enriched). Asterisks indicate whether there was a significant difference between the control and a particular treatment (Unpaired t-test, $\alpha=0.05$ ). The letters indicate whether there is a significant difference between groups (2-way ANOVA, followed by a Tukey post hoc, $\alpha=0.05$ for graphs A, B, C, D) (KruskalWallis test, followed by a Dunn's multiple comparison test post hoc, $\alpha=0.05$ for graph E). Controls (blue) were kept at $25^{\circ} \mathrm{C}$ for the entire experimental period and treatments (orange) were exposed to a temperature increase from $25-34^{\circ} \mathrm{C}$. 'Ambient', 'imbalanced' and 'enriched' represent different nutrient regimes. Values presented are the mean $+/$ - standard error. Details of statistical tests are in appendix section A2.1. 


\section{Oxygen flux measurements}

There was a significant interaction between temperature and nutrient regime on gross photosynthetic rate (two-way ANOVA, interaction, $\mathrm{f}(2,17)=6.638, \mathrm{p}=0.007$ ). There was no difference in rate between nutrient regimes at $25^{\circ} \mathrm{C}$, or any effect of temperature under an imbalanced or enriched regime (Figure $3.4 \mathrm{~A}$ ). However, cultures under the control nutrient regime had a $27 \%$ higher gross photosynthetic rate at $34^{\circ} \mathrm{C}$ than at $25^{\circ} \mathrm{C}$ (unpaired t-test, $\mathrm{p}=0.045$ ), which was also higher (by 23 and $59 \%$, respectively) than the rate under both an imbalanced and enriched nutrient regime at $34^{\circ} \mathrm{C}$ (Tukey's post hoc, $\mathrm{p}=0.01, \mathrm{p}<0.0001$ ).

Both temperature and nutrient regime significantly affected respiration rate, with the effect of temperature being dependent on nutrient availability (two-way ANOVA, interaction, $\mathrm{f}(2,17)=10.17$, $\mathrm{p}=0.001$ ). There was no significant difference between the respiration rates measured at the control temperature, irrespective of nutrient treatment (Figure 3.4 B). However, at elevated temperature, the cultures under the ambient nutrient regime had significantly higher respiration rates than those under imbalanced and nutrient-enriched regimes (Tukey's post hoc, $\mathrm{p}=0.004, \mathrm{p}-<0.0001$, respectively). Within each nutrient regime, respiration rate increased with temperature. The cultures under ambient nutrient level had a dramatically elevated respiration rate at $34^{\circ} \mathrm{C}$, which was $74 \%$ greater than the rate at $25^{\circ} \mathrm{C}$ (unpaired t-test, $\mathrm{p}=0.002$ ). Similarly, the cultures under an imbalanced nutrient regime had a $68 \%$ higher respiration rate at $34^{\circ} \mathrm{C}$ versus $25^{\circ} \mathrm{C}$. Interestingly, this effect of temperature on respiration rate was not as pronounced in cultures under an enriched nutrient regime, with no significant difference between rates at 25 and $34^{\circ} \mathrm{C}$.

Both temperature and nutrient regime significantly affected the gross photosynthesis to respiration ratio $(\mathrm{P}: \mathrm{R})$, with the effect of temperature being dependent on nutrient availability (two-way ANOVA, interaction, $\mathrm{f}(2,17)=5.63, \mathrm{p}=0.01)$. Regardless of nutrient regime, at $34^{\circ} \mathrm{C}$ there was a dramatic decrease in the P:R ratio, by $51-61 \%$, relative to the control temperature (unpaired t-test, 'ambient' and 'enriched' $\mathrm{p}=0.0001$, 'imbalanced' $\mathrm{p}=0.04$ ) (Figure $3.4 \mathrm{C}$ ). Interestingly, at $25^{\circ} \mathrm{C}$ the $P: R$ ratio of cultures under ambient and imbalanced nutrient regimes was higher than that under nutrient-enriched condition, by 18 and $25 \%$ respectively (Tukey's post hoc, $\mathrm{p}=0.01, \mathrm{p}=0.0002$ ). 

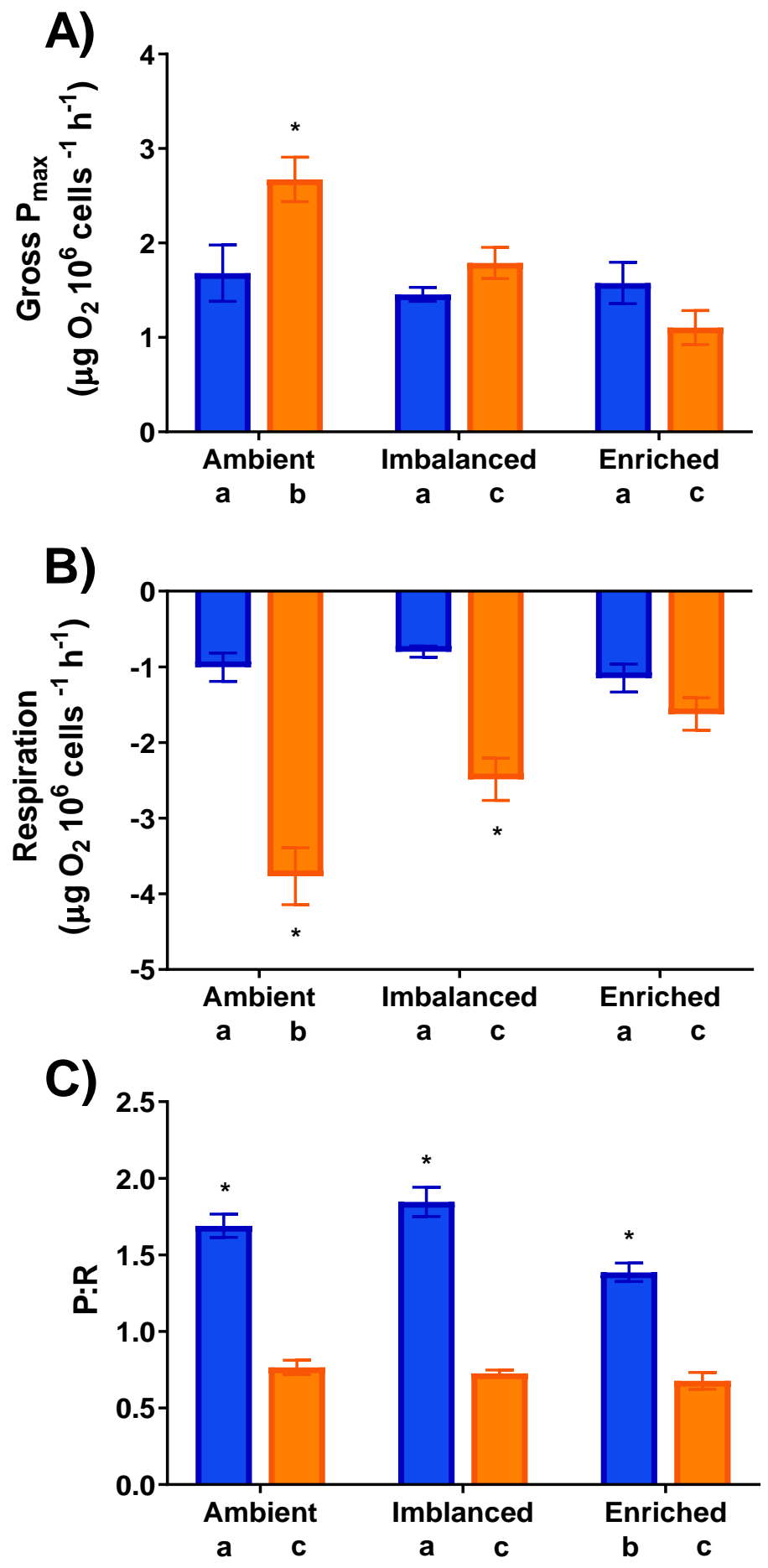

Figure 3.4: Maximum rates of cell-specific photosynthetic oxygen evolution and dark respiratory consumption per hour for cultures exposed to different temperatures and nutrient regimes. Controls (blue) were kept at $25^{\circ} \mathrm{C}$ for the entire experimental period and treatments (orange) were exposed to a temperature increase from $25-34^{\circ} \mathrm{C}$. 'Ambient', 'imbalanced' and 'enriched' represent different nutrient regimes. A) Maximum respiration rate; B) Maximum gross photosynthetic rate; D) The ratio of maximum gross photosynthesis to respiration. Values presented are the mean $+/$ - standard error (n $=4$ for all except 'ambient' controls for which $n=3$ ). The asterisks indicate whether within a nutrient regime there was a significant difference between the control and temperature treatment (unpaired ttest, $\alpha=0.05$ ). The letters indicate whether there was a significant difference between all groups (2way ANOVA, followed by Tukey's post hoc, $\alpha=0.05$ ). Details of statistical tests are in appendix section A2.1. 


\subsubsection{Proteomics analysis}

The predicted interaction between temperature and nutrient availability on the $B$. minutum proteome was not present (PERMANOVA, $\mathrm{p}=0.667$ ). However, separately, both temperature $(\mathrm{f}(1)=3.96, \mathrm{p}=$ $0.001)$, and nutrient regime $(f(2)=2.84, p=0.001)$ had a strong effect on the Symbiodiniaceae proteome. This is reflected in the PCA plot presented in Figure 3.5. There is a vertical separation driven by temperature and a horizontal separation driven by nutrient regime, showing grouping of the ambient and enriched treatments, but the separation of the imbalanced nutrient treatment.

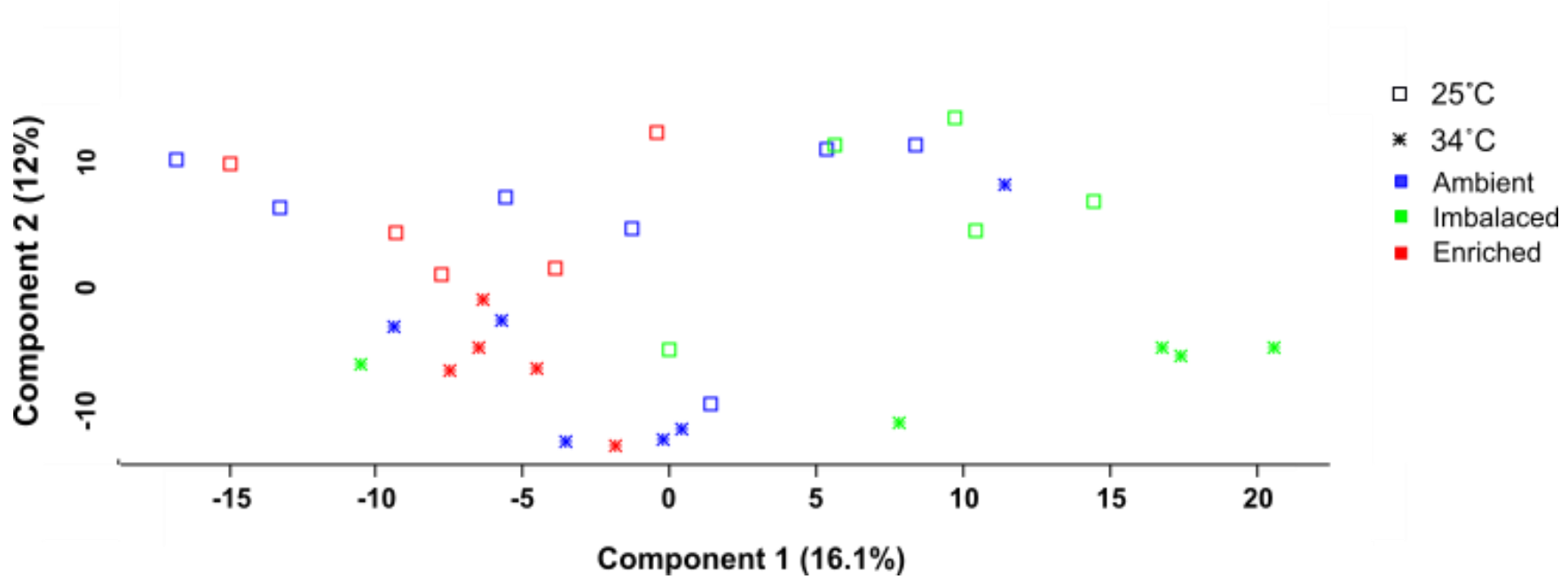

Figure 3.5: Principal component analysis of label-free quantification protein intensities. Each data point represents a biological replicate. Asterix represent cultures that were at $34{ }^{\circ} \mathrm{C}$, while squares represent cultures that were at $25^{\circ} \mathrm{C}$. Blue data points: cultures under an ambient nutrient regime, green data points: cultures under an imbalanced nutrient regime, red data points: cultures under an enriched nutrient regime.

\section{Differentially abundant proteins between treatments}

A total of 988 proteins were matched to proteins in the Uniprot KB database. Of these, 150 were differentially abundant (FDR, $q<0.1$ ) between B.minutum cultures at $25^{\circ} \mathrm{C}$ versus $34^{\circ} \mathrm{C}$. Alternately, protein abundance differences between nutrient regimes depended on which regimes were compared. There were merely 11 proteins differentially abundant between the ambient and enriched regimes. Alternately, when the imbalanced nutrient regime was compared with the ambient and enriched regime there were respectively 111 and 206 proteins differentially abundant (Figures 3.6 and 3.7). 
Notably, 24\% of all matched proteins from this analysis are uncharacterised, meaning that their function is not yet determined. This reflects a lack of dinoflagellate protein functional analysis, which restricts inferences that can be made from this data set.

Table 3.2: Number of differentially abundant proteins between the different thermal and nutrient regimes, based on false discovery rates $(0.05,0.1)$.

\begin{tabular}{l|lll} 
Treatment & $\mathbf{3 4}^{\circ} \mathbf{C}$ & Ambient & Enriched \\
\hline $\mathbf{2 5}{ }^{\circ} \mathbf{C}$ & 104,150 & & \\
Ambient & & & 2,11 \\
Imbalanced & & 42,111 & 106,206
\end{tabular}

\section{Temperature}

85 proteins had a significantly reduced abundance at $34^{\circ} \mathrm{C}$ relative to $25^{\circ} \mathrm{C}$, and 65 proteins had a relatively increased abundance at $34^{\circ} \mathrm{C}$ (Figure 3.6).

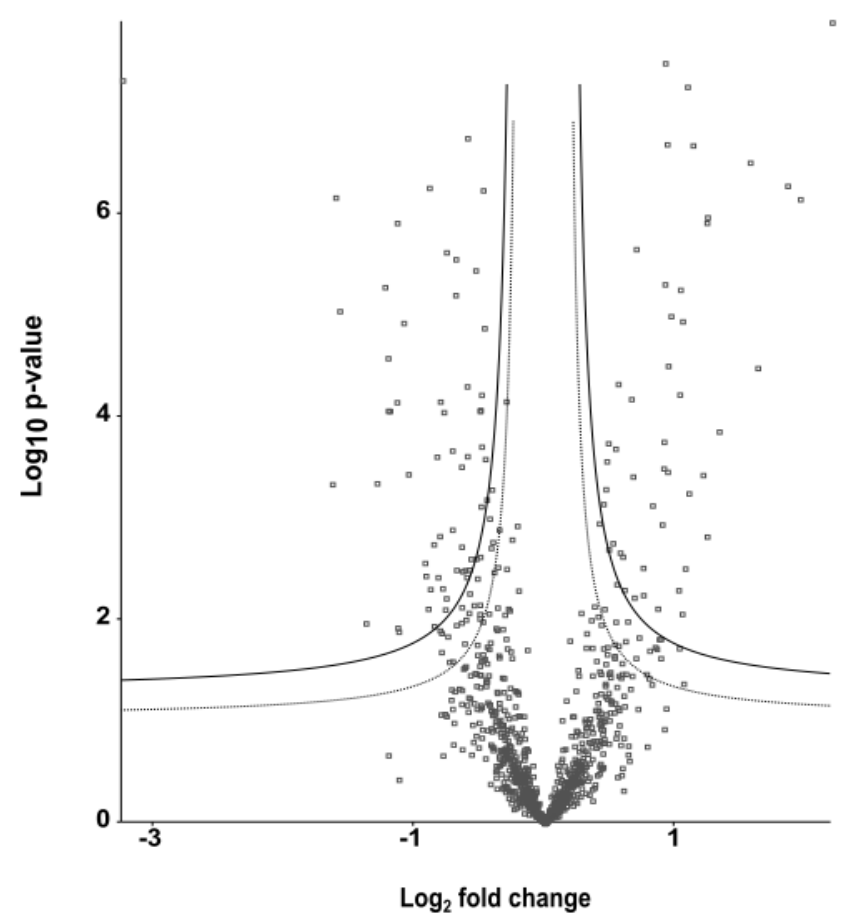

Figure 3.6: Quantitative proteomic analysis of Symbiodiniaceae cultures at 25 versus $34^{\circ} \mathrm{C}$. Each point represents one protein. The lower significance line $=0.1$ false discovery rate (FDR), and the upper significance line $=0.05$ FDR. Proteins to the left above the significance line are depleted at $34^{\circ} \mathrm{C}$ (relative to $25^{\circ} \mathrm{C}$ ) and proteins to the right of the significance line are upregulated at $34^{\circ} \mathrm{C}$. 


\section{Nutrient regime}

The proteome of $B$. minutum under a nutrient-enriched versus ambient nutrient regime was similar (Figure 3.7 A). Only seven proteins had a reduced abundance under the enriched regime relative to the ambient regime, while four proteins had a relatively higher abundance under the nutrient-enriched regime.

The proteome of $B$. minutum under the enriched nutrient regime was divergent from the proteome under the imbalanced regime (Figure 3.7 B). Under the imbalanced nutrient regime, 79 proteins had a relatively reduced abundance, and 127 proteins had an increased abundance, compared to the nutrientenriched regime. Notably, $28 \%$ or 57 of these differentially abundant proteins are uncharacterised.

The proteome of $B$. minutum under the ambient nutrient regime was also divergent from the proteome under the imbalanced regime (Figure 3.7 C), but less so than the nutrient-enriched regime, as there were $46 \%$ fewer differentially abundant proteins. Under the imbalanced nutrient regime, 35 proteins had a relatively reduced abundance, and 76 proteins had an increased abundance, compared to the ambient regime. Notably, $23 \%$ of these differentially abundant proteins are uncharacterised.

Of the proteins with significantly different abundances under an imbalanced nutrient regime versus the ambient and nutrient-enriched regimes, 69 had the same identity across these comparisons (32 with reduced abundance, 37 with increased abundance). However, a significant portion of the differentially abundant proteins had different identities depending on whether the imbalanced regime was compared with the ambient or nutrient-enriched condition (42\% and $80 \%$ of the total number of differentially expressed proteins, respectively) (Figure 3.7 B\&C). 
A)

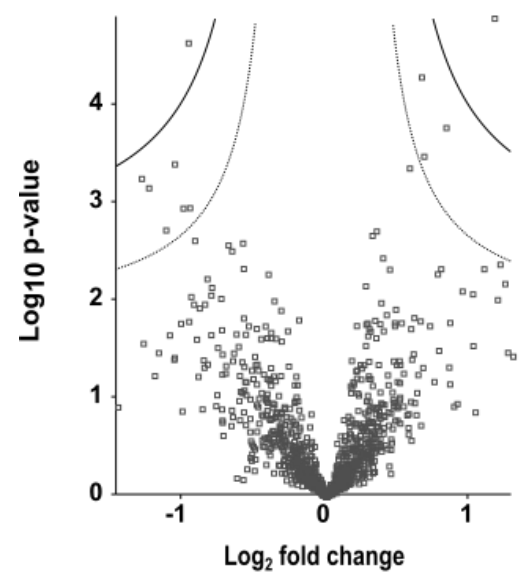

B)

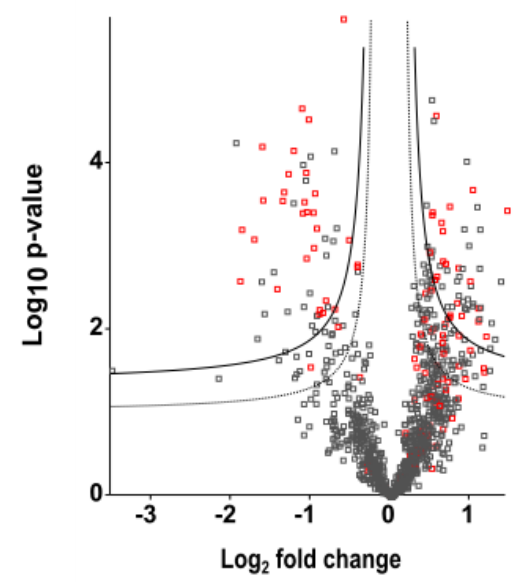

C)

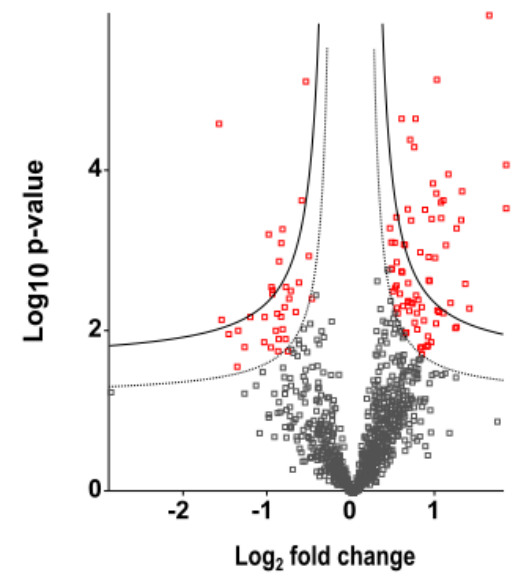

Figure 3.7: Quantitative proteomic analysis of Symbiodiniaceae cultures grown under different nutrient regimes. Each point represents one protein. (A) Ambient versus enriched nutrient regimes, with proteins to the left above the significance line being depleted under an enriched nutrient regime (relative to an ambient nutrient regime), and proteins to the right of the significance line being upregulated under the enriched nutrient regime. (B) Enriched versus imbalanced nutrient regimes, with proteins to the left above the significance line being depleted under an imbalanced (relative to an enriched nutrient regime), and proteins to the right of the significance line being upregulated under an imbalanced nutrient regime. (C) Ambient versus imbalanced nutrient regimes, with proteins to the left above the significance line being depleted under an imbalanced (relative to an ambient nutrient regime), and proteins to the right of the significance line being upregulated under an imbalanced nutrient regime. The proteins coloured red have a differential abundance between the 'ambient' and 'imbalanced' nutrient regime. The lower significance line $=0.1 \mathrm{FDR}$, and the upper significance line $=0.05 \mathrm{FDR}$.

\section{Functional analysis of differentially abundant proteins}

\section{Temperature}

There were more functional associations between the differentially abundant proteins of $B$. minutum cultures at 25 and $34{ }^{\circ} \mathrm{C}$ than expected by chance $\left(\mathrm{p}<1.0 \mathrm{e}^{-16}\right.$ ) (Figure 3.9). Unsurprisingly at $34{ }^{\circ} \mathrm{C}$, there was an enrichment in proteins involved in the cellular response to stress $\left(F D R=3.01 \mathrm{e}^{-09}\right)$, and protein folding $\left(\mathrm{FDR}=1.12 \mathrm{e}^{-14}\right.$ ) (Figure 3.9). Specifically, 23 chaperonin-like proteins and three proteins involved in cellular redox homeostasis were upregulated at $34^{\circ} \mathrm{C}$ (Figure 3.8). There was also enrichment in proteins with predicted functions of protein processing in the endoplasmic reticulum (FDR $=0.0008$ ). In relation, the abundance of proteins that mediate protein sorting and transport was altered by temperature (one down-regulated, three upregulated). From a metabolic 
perspective, there was a reduction in both energy producing and consuming pathways. There was enrichment in photosynthesis $\left(F D R=1.19 \mathrm{e}^{-15}\right)$, with photosynthetic light harvesting proteins, electrons transport proteins, and Calvin cycle proteins showing a reduced abundance at $34{ }^{\circ} \mathrm{C}(15$ and five, respectively) (See table S1, appendix A2.2). Interestingly, there was also enrichment in translation $\left(F D R=3.83 \mathrm{e}^{-05}\right.$ ), with 1 protein upregulated and 8 proteins down-regulated at $34{ }^{\circ} \mathrm{C}$. There was also enrichment in the energy generating pathways of glycolysis/gluconeogenesis (FDR = 0.0186), and the tricarboxylic acid (TCA) cycle (FDR $=0.0398)$, with the proteins that mediate these processes largely down-regulated at $34^{\circ} \mathrm{C}$ (see Figure 3.8).

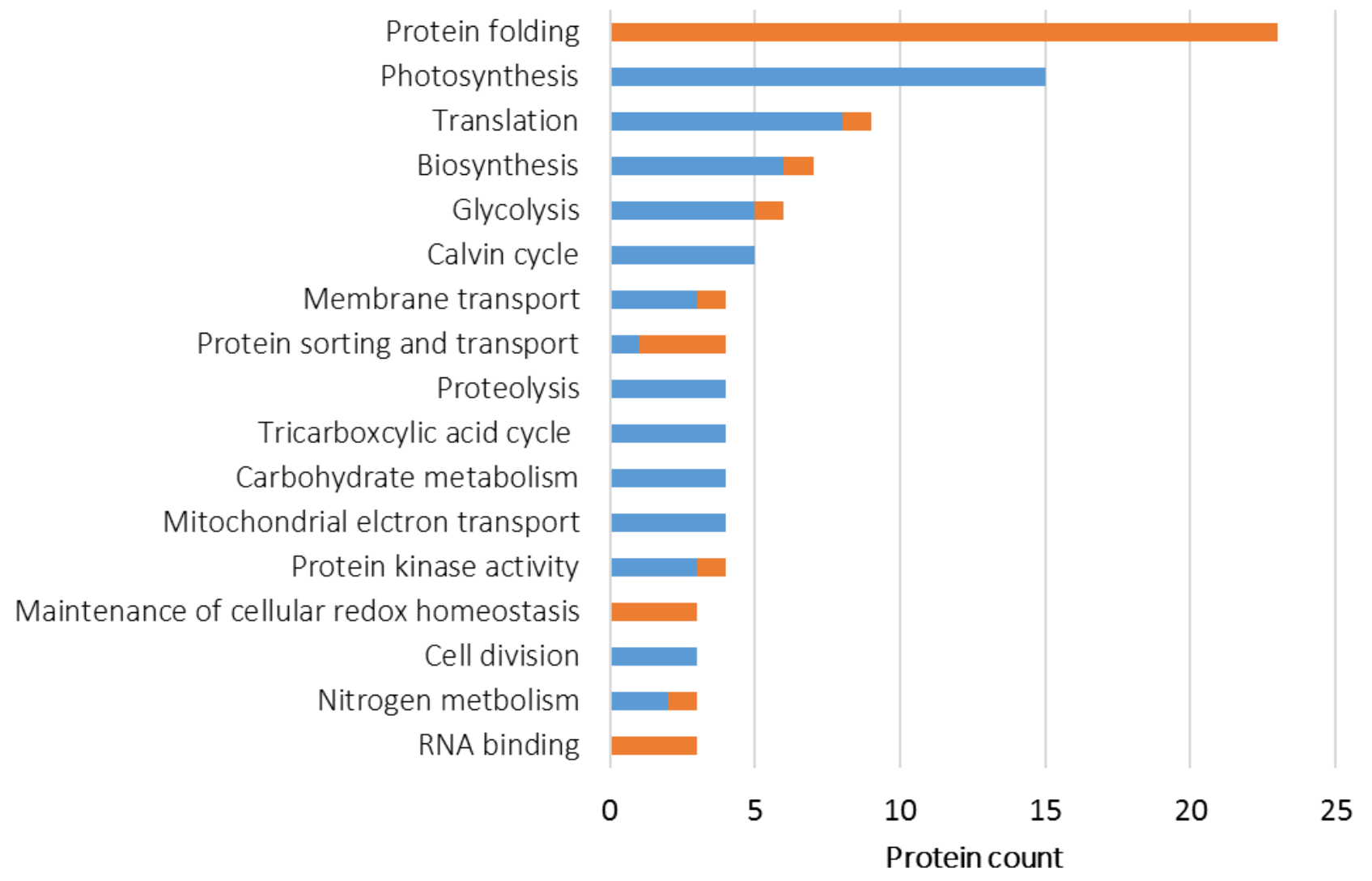

Figure 3.8: Counts of proteins that were differentially abundant $(\mathrm{p}<0.05)$ between control $\left(25^{\circ} \mathrm{C}\right)$ and heat-treated $\left(34^{\circ} \mathrm{C}\right) \mathrm{B}$. minutum, grouped by biological process gene ontology terms. Blue: down regulated at $34^{\circ} \mathrm{C}$ relative to $25^{\circ} \mathrm{C}$. Orange: upregulated at $34^{\circ} \mathrm{C}$ relative to $25^{\circ} \mathrm{C}$. Only biological process groups with three or more differentially abundant proteins are shown. 

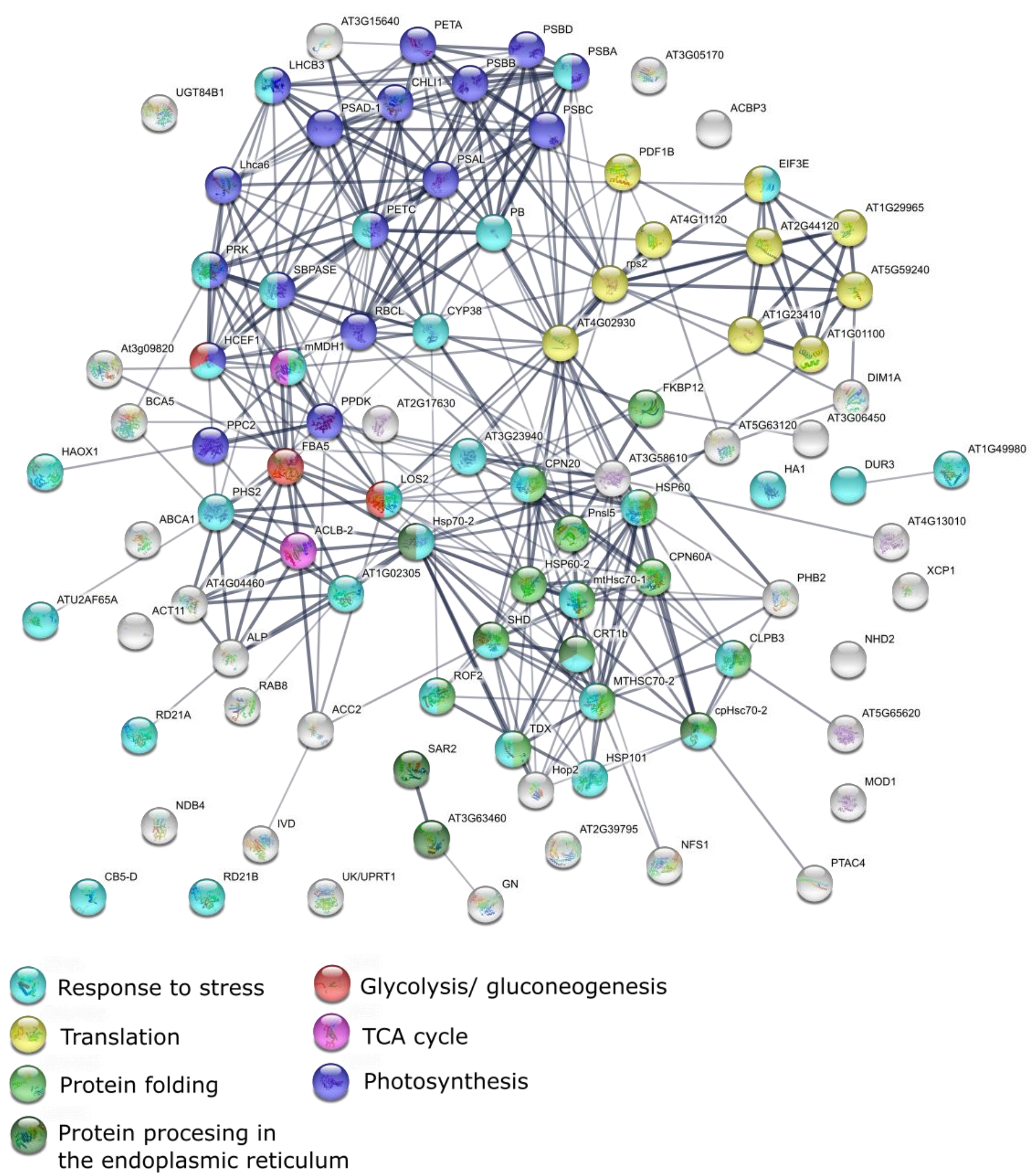

Figure 3.9: Protein interaction network analysis of differentially abundant proteins between 25 and $34{ }^{\circ} \mathrm{C}$ in Breviolum minutum. Interactions are based upon functional associations, with the thickness of connecting lines indicating the confidence in this interaction. Proteins are represented by nodes and colour-coded based on their predicted biological process or pathway ( $\mathrm{p}$-values given in the text). 


\section{Nutrient regime}

\section{Enriched versus Imbalanced}

There were more functional interactions between the set of differentially abundant proteins of $B$. minutum under the enriched and imbalanced nutrient regimes than expected by chance $\left(\mathrm{p}=2.01 \mathrm{e}^{-10}\right)$ (Figure 3.11). Interestingly, there was an enrichment of proteins involved in the cellular response to oxidative stress $(\mathrm{FDR}=0.0402)$ and cellular redox homeostasis $(\mathrm{FDR}=0.0052)$, with eight proteins down-regulated and four proteins up-regulated under an imbalanced nutrient regime. There was also an enrichment of proteins that mediate mRNA metabolic processes $\left(F D R=3.38 \mathrm{e}^{-05}\right)$, ribosome biogenesis $\left(\mathrm{FDR}=7.86 \mathrm{e}^{-07}\right)$, translation $\left(\mathrm{FDR}=4.66 \mathrm{e}^{-10}\right)$, and proteolysis $(\mathrm{FDR}=0.0402)$. Almost all of the proteins involved in these processes were upregulated under the imbalanced regime (see Figure 3.10), indicating a strong influence of nutrient availability on protein synthesis and repair. There was also an upregulation of five proteins involved in protein transport. Overall there was a strong enrichment in metabolic pathways $\left(F D R=8.00 \mathrm{e}^{-07}\right)$. Both glycolysis/gluconeogenesis $\left(\mathrm{FDR}=2.82 \mathrm{e}^{-}\right.$ $\left.{ }^{05}\right)$, and the TCA cycle $\left(\mathrm{FDR}=2.82 \mathrm{e}^{-05}\right)$ proteins were enriched, showing an upregulation under the imbalanced nutrient regime (Figure 3.10). In addition, there was an upregulation of eight proteins involved in carbohydrate metabolism, and three proteins involved in carbohydrate transport (Figure 3.10). In contrast, there was a down-regulation of all 12 identified photosynthesis proteins (Figure 3.10). There was relatively weak enrichment in photosynthesis proteins ( $F D R=0.0260)$. This is because many of these proteins were not included in the protein interaction network analysis as the majority were peridinin and chlorophyll-binding proteins, which are not present in Arabidopsis (see table S2 appendix A2.2). 


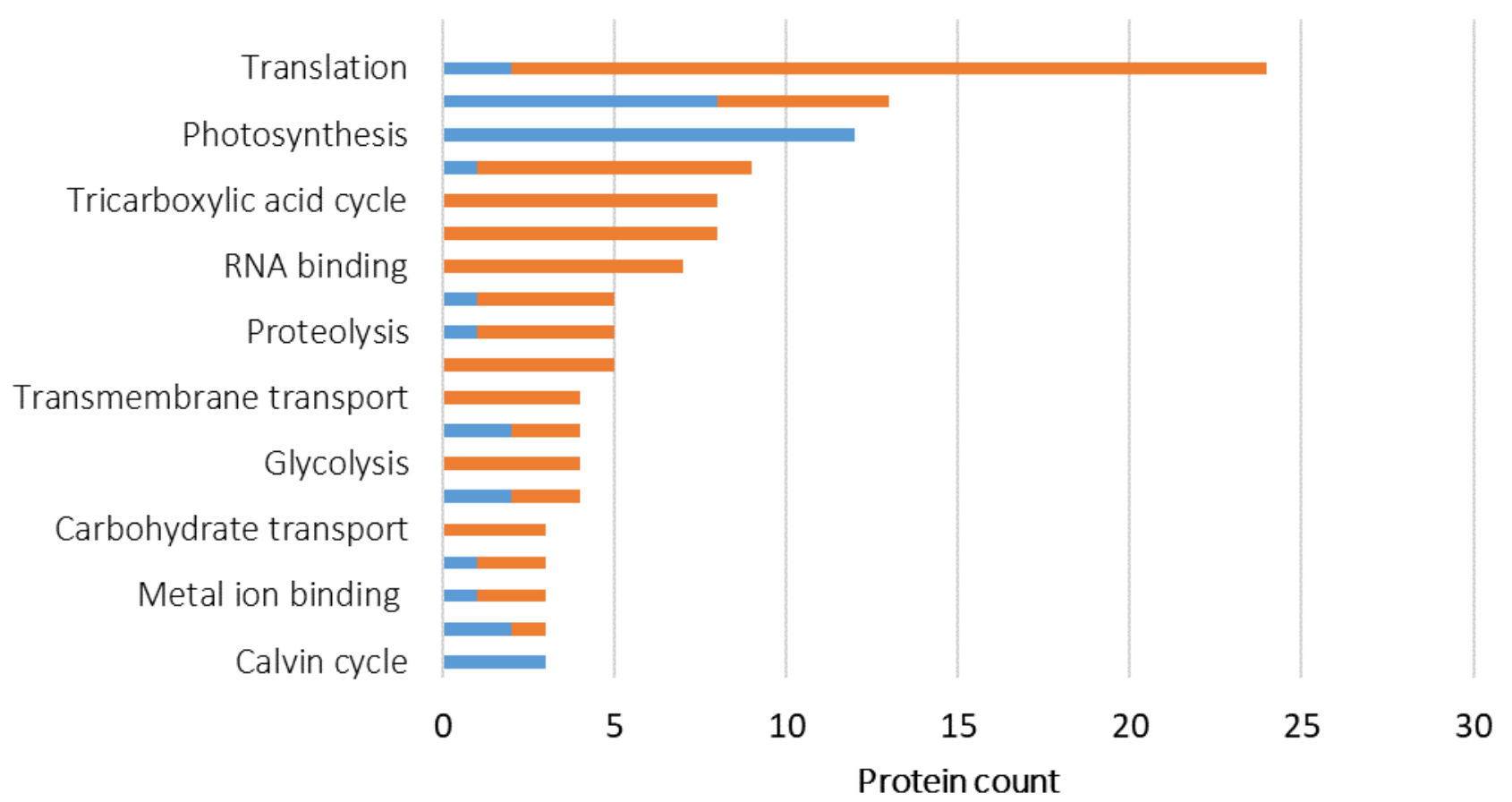

Figure 3.10: Counts of proteins that were differentially abundant $(\mathrm{p}<0.05)$ between Breviolum minutum under the enriched and imbalanced nutrient regimes, grouped by biological process gene ontology terms. 


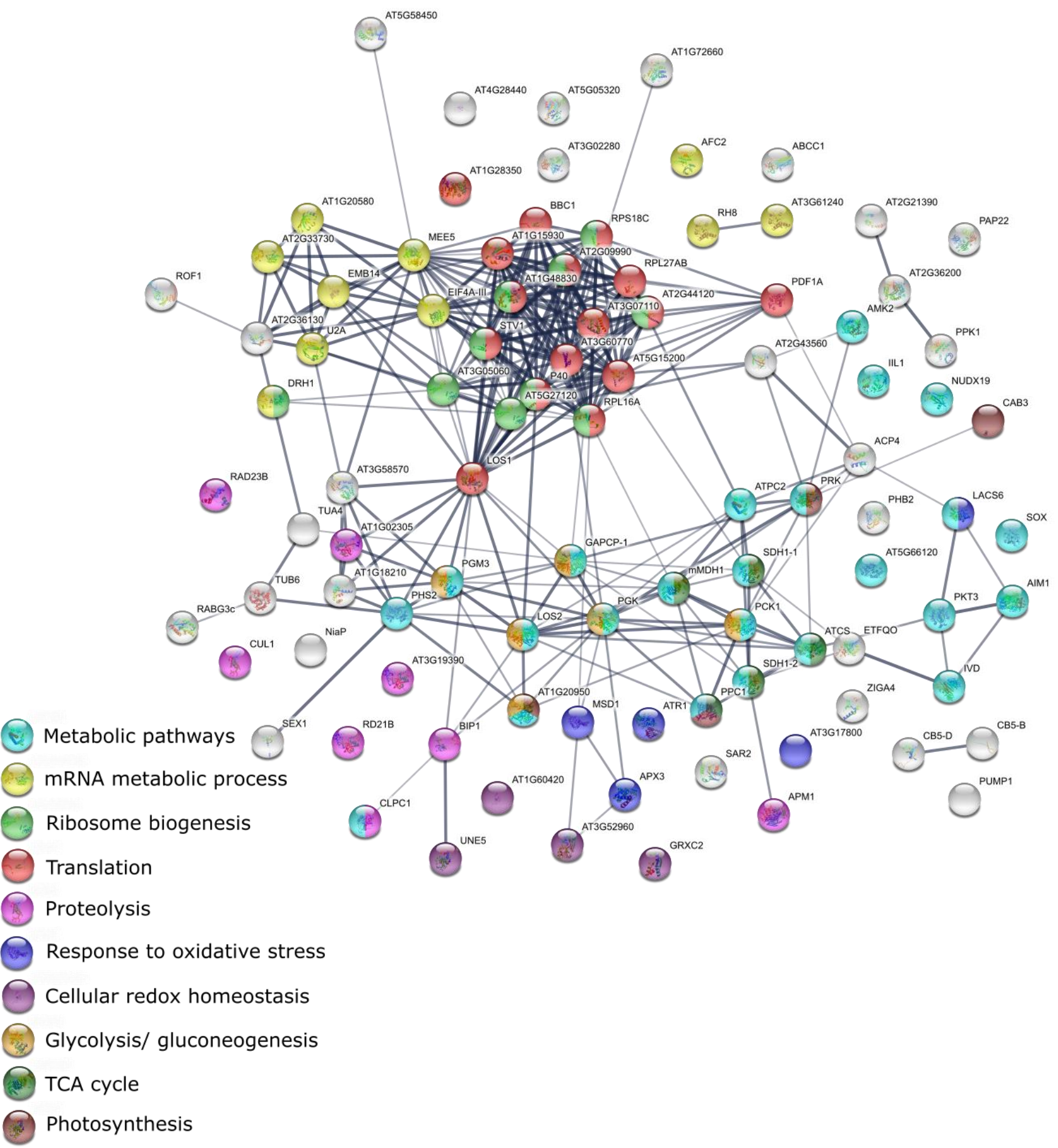

Figure 3.11: Protein interaction network analysis of differentially abundant proteins between an imbalanced and enriched nutrient regime. Interactions are based upon functional associations, with the thickness of connecting lines indicating the confidence in this interaction. Proteins are represented by nodes and colour-coded based on their annotated biological process or pathway ( $p$-values for this functional enrichment analysis are in text above). 


\section{Ambient versus imbalanced}

In contrast to the enriched regime, there were fewer differences between proteomes of cultures under the ambient and imbalanced nutrient regimes. This was reflected in fewer functional interactions between this smaller set of differentially abundant proteins. However, there were still more interactions than expected by chance $\left(\mathrm{p}=6.3 \mathrm{e}^{-4}\right.$ ) (Figure 3.13), and similar groups of proteins were affected. There was an enrichment in metabolic processes $\left(F D R=3.16 \mathrm{e}^{-09}\right)$, such as carbohydrate catabolism $\left(F D R=2.3 \mathrm{e}^{-4}\right)$. In relation, three proteins involved in glycolysis and five proteins involved in carbohydrate metabolism were upregulated under the imbalanced regime relative to the ambient regime (Figure 12). In alignment with the comparison between the enriched and imbalanced regimes, protein metabolism was also enriched $\left(F D R=7.2 \mathrm{e}^{-4}\right)$. Linked to this, there was enrichment in mRNA metabolic processes $\left(F D R=2.1 \mathrm{e}^{-3}\right)$, reflected by the up-regulation of three proteins involved in mRNA splicing via the spliceosome. There was also enrichment in the regulation of translation $\left(\mathrm{FDR}=1.6 \mathrm{e}^{-3}\right)$, linked to altered abundance of proteins that mediate translation under the imbalanced regime (two down-regulated, three up-regulated, Figure 3.12). In addition, there was an upregulation of three proteins that mediate protein transport and five proteins involved in proteolysis. There was also an enrichment of photosynthesis $\left(F D R=7.5 \mathrm{e}^{-3}\right)$, with the seven measured photosynthesis proteins down-regulated under the imbalanced regime (Figure 3.12). The largest group of proteins affected by the ambient versus imbalanced regime were those that mediate cellular redox homeostasis $\left(\mathrm{FDR}=1.3 \mathrm{e}^{-3}\right)$. Under the imbalanced nutrient regime four proteins were up-regulated and six proteins were down-regulated relative to the ambient regime (Figure 3.12). Overall, the cellular process affected by the imbalanced nutrient regime were similar whether this nutrient treatment was compared with the ambient or enriched treatments. 
Maintenance of cellular redox homeostasis

Photosynthesis

Carbohydrate metabolism

Proteolysis

Translation

ATPase activity

Protein modification

Cell division

Response to nutrient environment

Glycolysis

Protein transport

mRNA splicing, via the spliceosome

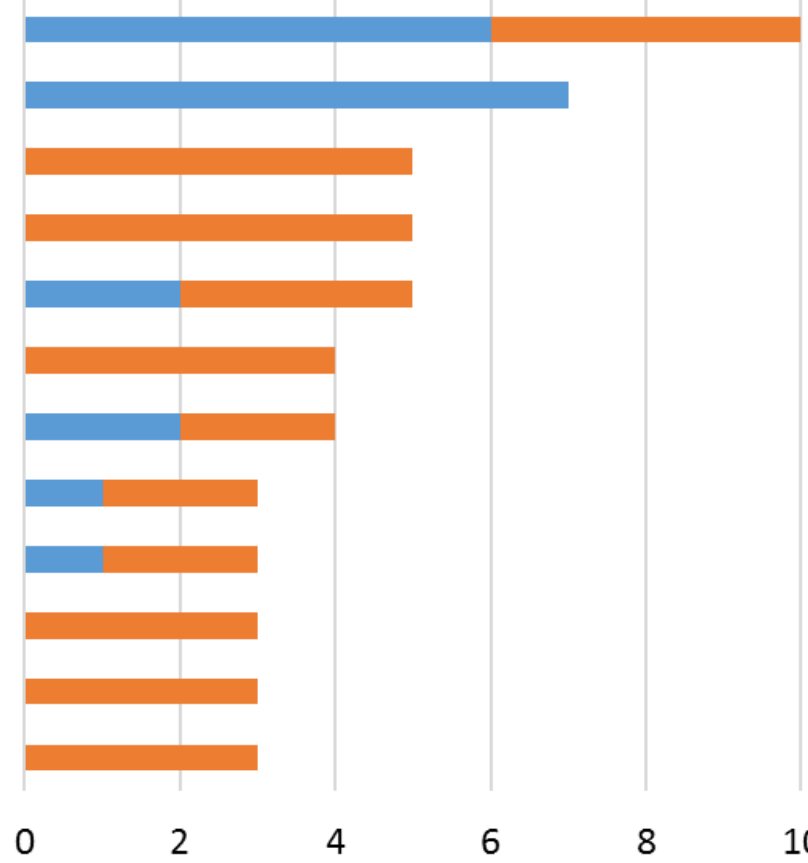

Protein count

Figure 3.12: Counts of proteins that were differentially abundant $(\mathrm{p}<0.05)$ between $B$. minutum under the ambient and imbalanced nutrient regimes, grouped by biological process and function gene ontology terms. 


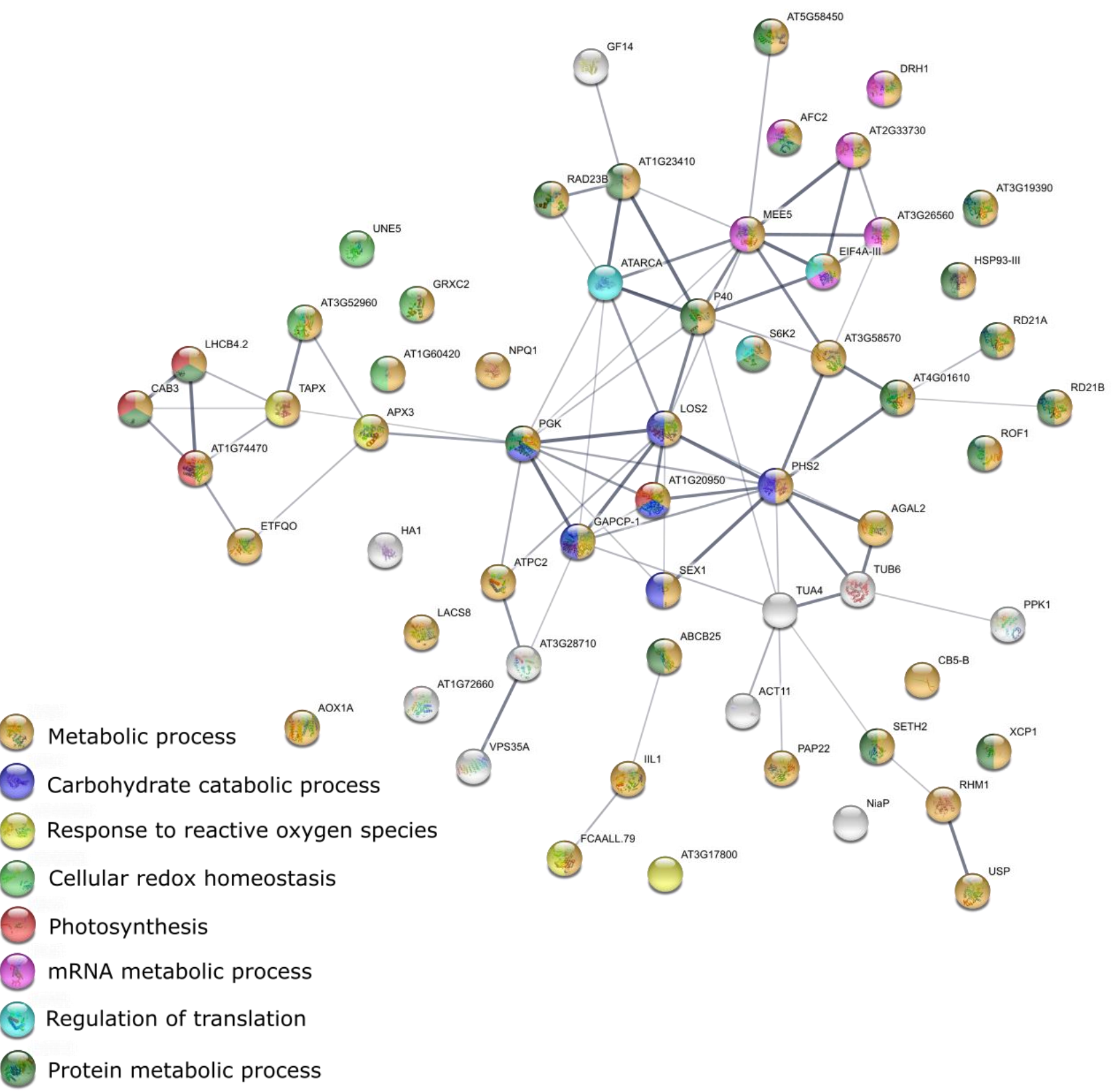

Figure 3.13: Protein interaction network analysis of differentially abundant proteins between an imbalanced and ambient nutrient regime. Interactions are based upon functional associations, with the thickness of connecting lines indicating the confidence in this interaction. Proteins are represented by nodes and colour-coded based on the biological process or pathway they are involved in (p-values for this functional enrichment analysis are in text above). 


\subsection{Discussion}

Although it was hypothesised that there would be an interaction between nutrient regime and the proteomic response to elevated temperature, this was not observed. However, individually both temperature and nutrient regime had a strong impact on the proteome, and thus are discussed separately.

\subsubsection{The proteomic response of Breviolum minutum to elevated temperature}

\section{Overview}

Temperature had a strong effect on the proteome of B. minutum across nutrient regimes. Overall, elevated temperature affected the expression of proteins in several ways: with a reduced abundance of photosynthesis proteins, ribosomal proteins, metabolic proteins (Calvin cycle/glycolysis) and proteins involved in biosynthesis, and an increased abundance of chaperonin proteins and proteins involved in cellular redox homeostasis.

\section{Photosystems}

It is well established that thermal stress causes photosynthetic dysfunction in Symbiodiniaceae (Warner et al. 1996, Weis 2008, Oakley and Davy 2018); accordingly, the quantum yields measured here declined with temperature, indicating thermally-induced photoinhibition (Genty et al. 1989, Warner et al. 1999). Interestingly, the rate of gross photosynthetic oxygen evolution was not significantly affected by temperature when cultures were under an imbalanced or enriched nutrient regime and was positively affected by temperature under the ambient nutrient regime. However, I identified a significant reduction in the abundance of all detected photosystem I and II proteins at $34^{\circ} \mathrm{C}$ relative to $25^{\circ} \mathrm{C}$. This discordance is likely driven by increased photosynthetic enzyme activity at elevated temperature (Peterson et al. 2007).

Interestingly, transcriptomic studies of Symbiodiniaceae under thermal stress have found variable expression of photosynthesis proteins, or no change at all (Barshis et al. 2014, Gierz et al. 2017). It is reasonable to assume that the discrepancy between these studies and the reduced photosynthetic protein abundance at elevated temperature found here is a consequence of post-translational changes in protein abundance (Okamoto et al. 2001, Bachvaroff and Place 2008, Leggat et al. 2011). As was 
observed in this proteomic analysis, previous studies have also identified a reduction of photosynthesis proteins with temperature when using immunoblot analysis and targeted PCR to measure the expression of genes which encode for a given protein (Takahashi et al. 2008, McGinley et al. 2012b, Gierz et al. 2016): specifically, the D1 protein which is part of the water-splitting complex in photosystem II (Takahashi et al. 2008, McGinley et al. 2012b, Gierz et al. 2016), and the light-harvesting antenna chlorophyll $a$-chlorophyll $c 2$-peridinin-protein complexes (acpPC) (Takahashi et al. 2008). Rubisco, which facilitates carboxylation within photosynthesis, has been found to reduce activity at elevated temperature (Lilley et al. 2010). The proteomic analysis identified a reduced abundance of this protein in response to elevated temperature, which has not previously been measured in Symbiodiniaceae due to the poor stability of this enzyme. Overall, the reduction in photosynthetic protein abundance likely reflects thermally-induced disintegration of photosynthetic proteins, possibly mediated by reactive oxygen species (ROS), in conjunction with the inhibition of photosystem repair via the de novo synthesis of proteins (Ohad et al. 1994, Takahashi et al. 2004, Takahashi and Murata 2005, Lesser 2006, Takahashi et al. 2008, Mathur et al. 2014). Supporting this hypothesis, evidence indicates that protein synthesis is impaired at elevated temperature (discussed in next section).

\section{Protein synthesis}

At $34{ }^{\circ} \mathrm{C}$, there was a reduction in ribosomal protein abundance relative to $25^{\circ} \mathrm{C}$ (see table $\mathrm{S} 1$ ), signifying possible thermally-induced ribosomal degradation and reduced protein synthesis at elevated temperature. Interestingly, at $34^{\circ} \mathrm{C}$ there was also a relative increase in abundance of a pentatricopeptide repeat-containing protein, which plays an unspecified role in translation (Hammani et al. 2011), and the 'eukaryotic translation initiation factor 3 (subunit) E' which has been found to inhibit translation in Arabidopsis (Yahalom et al. 2008). However, translation was still occurring in the current study, as there was still an increased abundance of specific proteins, such as chaperonin proteins (discussed in the next section). Consistent with this, analysis of mRNA translation in $A$. thaliana seedlings revealed a 50\% reduction in mRNA translation in response to thermal stress, including those involved in ribosome biogenesis (Yángüez et al. 2013). As was observed by direct analysis of protein abundance in this study, the transcriptomic study of Arabidopsis also identified a selective translation of mRNA encoding for stress response proteins, such as those involved in the response to oxidative stress along with heat shock proteins (Yángüez et al. 2013). Taken together, this suggests that, under thermal stress, Symbiodiniaceae may be redirecting resources away from protein synthesis and towards the maintenance of protein function and cellular homeostasis. 


\section{Chaperonin proteins}

A total of 23 heat-shock or chaperonin-like proteins exhibited a significantly higher abundance at 34 ${ }^{\circ} \mathrm{C}$ relative to $25^{\circ} \mathrm{C}$ (see table $\mathrm{S} 1$ ). Similarly, this trend has been observed across eukaryotic organisms from mammals to plants (Finka et al. 2011), including in transcriptomic analysis of Symbiodiniaceae (Rosic et al. 2011, Gierz et al. 2017). Chaperonins and heat-shock proteins mediate protein folding, assembly and translocation (Boston et al. 1996, Hartl 1996, Wang et al. 2004). Under stressful conditions, they act to stabilise membranes and sustain protein conformation and thus functionality, ultimately maintaining cellular homeostasis (Vierling 1991, Wang et al. 2004). For example, an (FKBP)-type immunophilin in Arabidopsis increases photosynthetic stress tolerance, possibly by maintaining the stability of the core photosystem I protein Psal. Interestingly, in the current study the most significantly upregulated protein in response to elevated temperature was a similar immunophilin, FKBP65, which also acts as a peptidyl prolyl isomerase. Overall, the significant upregulation of chaperonin and heat-shock proteins in B. minutum at elevated temperature represents a core defence mechanism against the dissociative effects of elevated temperature on cellular function.

\section{Redox homeostasis}

Thermal stress increases the production of ROS, causing cellular oxidative damage (Lesser 2006). Like other autotrophs, Symbiodiniaceae have a network of antioxidant enzymes and molecules which act to neutralise ROS (Bayer et al. 2012, Krueger et al. 2014, Hawkins et al. 2015). In this study, three oxidoreductases, which catalyse the removal of electrons from reactive species, had higher abundance at $34{ }^{\circ} \mathrm{C}$ ( see table S1, appendix section A2.2). In addition, the one glycolysis protein that exhibited increased abundance at $34{ }^{\circ} \mathrm{C}$, cystolic fructose-bisphosphate aldolase, may play a role in signal transduction involved in adaption to oxidative stress (Wojtera-Kwiczor et al. 2013). However, it was expected that there would be a greater abundance of differentially-expressed proteins involved in the oxidative stress response between control and treatment temperatures. Other studies have also measured an upregulation of antioxidants in Symbiodiniaceae in response to thermal stress (Krueger et al. 2014, Hawkins et al. 2015). The antioxidant response to elevated temperature varies between Symbiodiniaceae types, with thermally-sensitive types exhibiting a proportionally lower upregulation of antioxidants than thermally-tolerant types in response to elevated temperature (Krueger et al. 2014). This may partially explain the upregulation of so few antioxidants observed in this current study of B. minutum, which is considered to be a Symbiodiniaceae species of intermediate thermal 
tolerance (Swain et al. 2017). Interestingly, there was a considerably greater amount of differentiallyexpressed antioxidant proteins between nutrient regimes than there was between thermal treatments (discussed in section 3.4.2).

\section{Cellular function and metabolism}

Regardless of nutrient regime, cultures at $34{ }^{\circ} \mathrm{C}$ showed a negative metabolic balance (i.e. P:R $<1$ ). In particular, while there was no inhibitory effect of elevated temperature on gross photosynthetic rate, there was a trend of increased respiration rate with increased temperature, as has been observed previously in thermally stressed Symbiodiniaceae (Iglesias-Prieto et al. 1992, Buxton et al. 2009, Oakley et al. 2014b).

Interestingly, cell growth rate increased with elevated temperature under ambient and enriched nutrient conditions. However, the cell counts to calculate population growth were taken at the beginning and end of the experimental period. Thus, what may be driving this trend is an increase in growth rate at moderately elevated temperature $\left(25-30^{\circ} \mathrm{C}\right)$ rather than at a higher temperature (Karako-Lampert et al., 2005; Peterson et al., 2007). This may explain why, conversely, the proteomics data suggest a downregulation of cell division at $34^{\circ} \mathrm{C}$ relative to $25^{\circ} \mathrm{C}$. At elevated temperature there was a reduction in three proteins linked to mitotic division; most significantly the cyclin-dependent kinase regulatory subunit. This protein binds to the catalytic subunit of cyclindependent kinases, which control the progression of the cell cycle through the $G_{1} / S$ and $G_{2} / M$ phases (Malumbres and Barbacid 2005). Thus, the reduced abundance of this regulatory protein at elevated temperature would arrest the cell cycle, reducing rates of mitotic division.

It was hypothesised that, under thermal stress, the abundance of proteins involved in energygeneration pathways, specifically the TCA cycle and glycolysis, would increase, while those involved in energetically-costly cellular processes such as biogenesis would decrease, as has been inferred from metabolomic studies of Symbiodiniaceae (Hillyer et al. 2016a, Hillyer et al. 2017). Instead, there was a reduction in proteins involved in energy-producing pathways such as glycolysis, the TCA cycle, and the Calvin cycle, as well as energy-consuming pathways such as protein synthesis, fatty acid synthesis and general biosynthesis. Overall, this could simply reflect a breakdown of cellular function. It was surprising that, despite dramatically increased respiration rates, cytochrome $b 2$, cytochrome $c$ oxidase (subunit 5b-1), and external alternative $\mathrm{NAD}(\mathrm{P}) \mathrm{H}$-ubiquinone oxidoreductase (b1), which form part of the respiratory electron transport chain, had reduced abundances (see table S1, appendix section 
A2.2). This could be a consequence of increased enzyme activity at elevated temperature (Peterson et al. 2007). As protein abundance does not equal protein activity, performing physiological measurements in conjunction with proteomic analysis is important for making inferences about cellular physiology.

\subsubsection{The proteomic response of Breviolum minutum to nutrient availability}

\section{Overview}

The relative abundance of nitrogen to phosphorus had a strong influence on the Symbiodiniaceae proteome. There was a negligible difference between the proteome of cultures under the ambient or enriched regime. However, when comparing both of these regimes with the imbalanced nutrient treatment, marked differences were apparent in the Symbiodiniaceae proteome. Physiological and proteomic data indicated that the cultures under an imbalanced nutrient regime were experiencing phosphorus deficiency, as has been previously observed in Symbiodiniaceae under high levels of nitrogen relative to phosphorus (Wiedenmann et al. 2013, D'Angelo and Wiedenmann 2014, Rosset et al. 2017). Differential protein abundance suggested that under imbalanced nutrient levels there was a reduction in carbon fixation, with a concomitant increase in protein translation, storage product turn over, and energy generation pathways such as glycolysis and the TCA cycle (see Figure 3.14). There was also evidence of metabolic redundancy, whereby alternate enzymes where used to maintain cellular processes under reduced ATP availability. In addition, there was a significant up- and downregulation of multiple enzymes involved in redox homeostasis in response to nutrient availability, highlighting a previously unidentified link between nutrient availability and the antioxidant network worthy of further investigation. Despite the proteomic data illustrating multiple avenues of altered cellular physiology under an imbalanced nutrient regime, the photosynthetic efficiency and metabolic balance (i.e. P:R ratio) was not lower relative to measurements under the ambient or nutrient enriched regime. This suggests that, by altering metabolism and halting cellular growth, $B$. minutum was able to adapt its cellular physiology to maintain function and homeostasis under an imbalanced nutrient regime. 


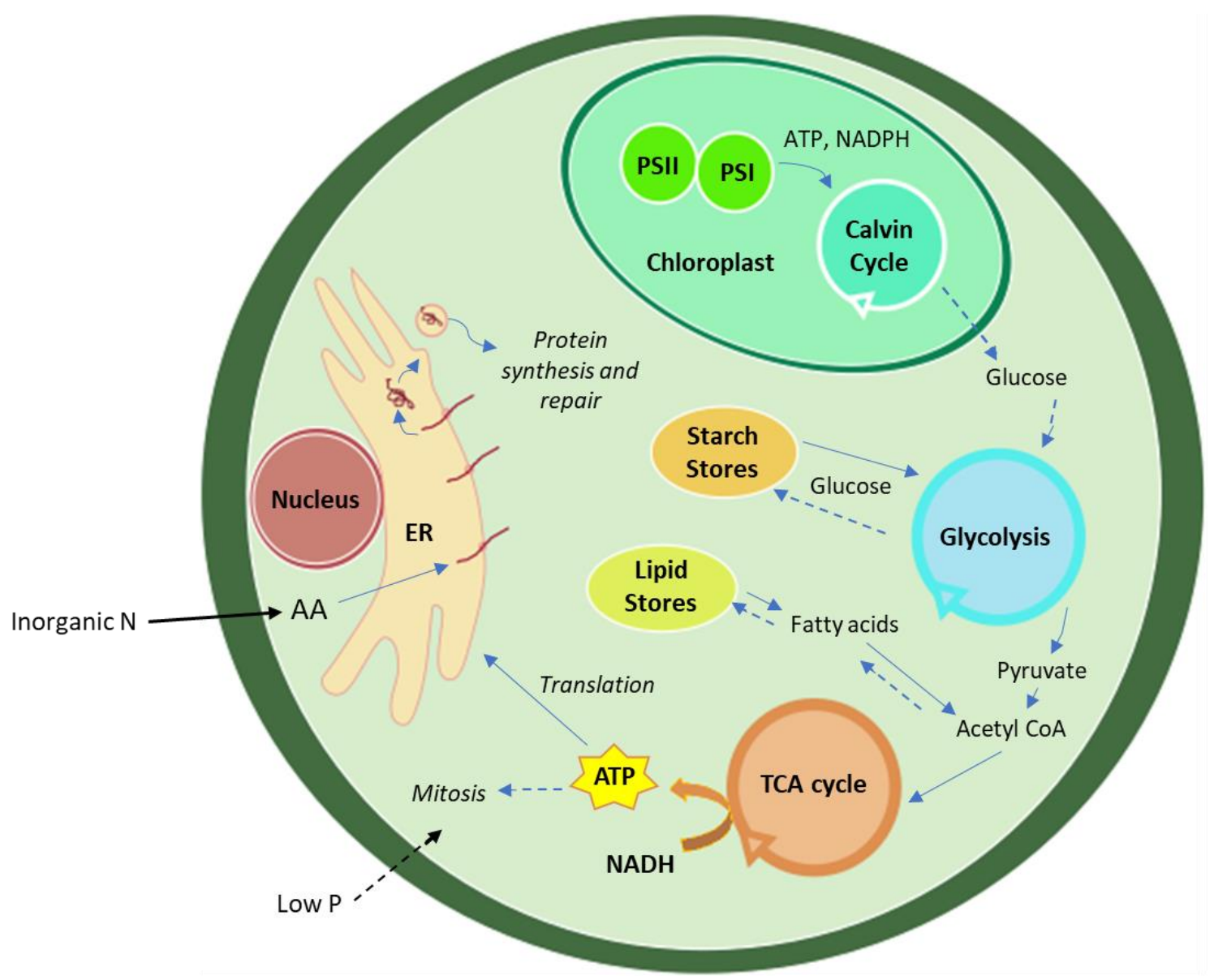

Figure 3.14: Conceptual diagram illustrating possible alterations of cellular physiology in B. minutum in response to an imbalanced nutrient regime. Reduced carbon fixation causes a reduction in photosynthate production and glucose for break down via glycolysis. Stored fatty acids and glucose are broken down by the TCA cycle and glycolysis in order to generate ATP. In conjunction, phosphate deficiency caused by an imbalanced nitrogen to phosphorus ratio halts mitosis decreasing energy demands. The high levels of nitrogen can be used to produce amino acids, which can be utilised in conjunction with the energy generated by the TCA cycle and glycolysis to increase protein translation, synthesis and repair. Dashed arrows indicate a reduction in that pathway. $\mathrm{ER}=$ endoplasmic reticulum, AA= amino acids, $\mathrm{PSII}=$ photosystem $\mathrm{II}, \mathrm{PSI}=$ photosystem $\mathrm{I}$

\section{Nutrient assimilation}

The abundance and activity of proteins that mediate nutrient assimilation and conversion were affected by nutrient availability. There was an increase in the concentration of type- 3 glutamine synthetase and nitrate reductase under an imbalanced regime compared to the ambient and enriched regimes, respectively (see tables $\mathrm{S} 2$ and $\mathrm{S} 3$, appendix section A2.2). Both of these enzymes are 
involved in nitrogen uptake (Wilkerson and Trench 1985, Anderson and Burris 1987). The specific enzymes involved in phosphate uptake in Symbiodiniaceae have not been characterised.

Interestingly, the abundance of purple acid phosphatase was reduced under an imbalanced nutrient regime relative to the enriched regime. Purple acid phosphatases are a large class of enzymes, which are generally thought to recycle inorganic phosphorus from intracellular organic compounds (Barlow and Triemer 1986, Plaxton and Tran 2011), but have also been implicated in cell wall synthesis and the metabolism of ROS in higher plants (Del Pozo et al. 1999, Kaida et al. 2009).

The activity of alkaline phosphatase (APA), which converts extracellular organic phosphorus into inorganic phosphorus allowing uptake (Doonan and Jensen 1980, Vincent and Crowder 1995), was measured in this study. APA was highest under the imbalanced nutrient treatment, even though the levels of available phosphorus were similar to the enriched regime and lower under the ambient nutrient regime. An increase in APA activity is a conserved response to phosphate deficiency in marine autotrophs (Møller et al. 1975, Pérez and Romero 1993), including Symbiodiniaceae (Annis and Cook 2002, Wiedenmann et al. 2013). Thus, the relative abundance of nitrogen to phosphorus may be causing phosphate deficiency under an imbalanced nutrient regime, as has been previously observed in Symbiodiniaceae (Wiedenmann et al. 2013, D’Angelo and Wiedenmann 2014, Rosset et al. 2017). Interestingly, alkaline phosphatase wasn't detected in the proteomics results, possibly because it may be below the detection threshold, and/ or because it is associated with outer cellular membranes (Doonan and Jensen 1980), which is depleted in our sample protocol as cellular debris are pelleted out of solution.

\section{Photosynthesis}

Photosystem proteins had a reduced abundance under an imbalanced nutrient regime compared to the ambient and enriched nutrient regimes. Most of these proteins were either part of the light-harvesting complex or the photosynthetic electron transport chain (see tables S2 and S3, appendix section A2.2). In addition, the relative abundance of Calvin cycle enzymes, which mediate photosynthetic carbon fixation, had a reduced abundance. This may reflect a reduction in chloroplast size relative to the entire cell size. Interestingly, chlorophyll $a$ and carotenoid concentration per cell did not decrease under the imbalanced nutrient regime. However, this measurement does not take into account cell size, which has been found to increase in dinoflagellates, including Symbiodiniaceae under phosphate starvation (Li et al. 2016, Rosset et al. 2017). This increase in cell size is thought to be driven by 
continued carbon fixation facilitating cellular growth while phosphorus deficiency inhibits DNA duplication or checkpoint protein phosphorylation, halting mitosis (Li et al. 2016).

There was no substantial effect of nutrient availability on photosynthetic efficiency. Similarly, previous studies have found quantum yield to be insensitive to nutrient availability in both diatoms and Symbiodiniaceae in hospite (Parkhill et al. 2001, Wiedenmann et al. 2013). Gross photosynthetic rates were also unaffected by nutrient availability at the control temperature. However, relative to the ambient and enriched nutrient regimes, the Symbiodiniaceae under the imbalanced regime had severely depressed population growth, likely reflecting phosphorus limitation, as nitrogen levels were high (Rosset et al. 2017). Taken together, the maintained photosynthetic activity and limited growth indicate an uncoupling between carbon fixation and cell division. One interpretation of this is that, by decreasing energy demands by halting mitosis, it may be possible to sustain photosynthesis (Li et al. 2016, Rosset et al. 2017).

\section{Protein translation}

The proteomic evidence suggests increased protein translation under an imbalanced nutrient regime. Compared to an ambient and enriched nutrient regime, there was a relative increased abundance of proteins involved in RNA binding and mRNA processing, splicing and transport, in addition to numerous ribosomal proteins (see tables S2 and S3). There was a greater number of differentiallyabundant proteins linked to translation between the imbalanced and enriched conditions. In addition to the functional groups described above, there was an increased abundance of proteins involved in ribosomal biogenesis (Nucleolar protein 56 and Nucleolar protein 5-1), along with tRNA adenine(37)N6-methyltransferase and tyrosine tRNA ligase, which aid in the process of tRNA decoding of mRNA and joining the appropriate amino acid to the tRNA anti-codon (Qian et al. 1998, Bonnefond et al. 2005). There was also an increased abundance of Elongation Factor 2, which catalyses the GTPdependent ribosomal translocation step (Jørgensen et al. 2006), and Elongation Factor 1-alpha, which promotes tRNA binding to the ribosome (Visweswaraiah et al. 2011). In addition, there was an upregulation of proteins involved in intracellular protein transport, including coatomer subunit alpha2 and GTP-binding protein SAR1A, which are involved in the transport of newly synthesised proteins from the endoplasmic reticulum to the Golgi body (Pimpl et al. 2000, Hwang and Robinson 2009). Furthermore, there was an increased abundance of proteins involved in proteolysis under an imbalanced nutrient regime, possibly reflecting the recycling of protein building-blocks. Nitrogen is an essential component of amino acids, which are the building blocks of proteins (Bothe et al. 2006). 
The high levels of nitrogen under the imbalanced nutrient regime may have supported an increase in translation. An increase in protein production under an imbalanced nutrient condition may reflect an adaptive response, whereby Symbiodiniaceae are producing the necessary proteins to alter cellular physiology to maintain homeostasis (see Figure 3.14).

Interestingly, the chloroplastic Pentatricopeptide repeat-containing protein, which positively regulates chloroplast RNA processing and translation (Zoschke et al. 2013), had a reduced abundance under the imbalanced nutrient regime relative to the ambient and enriched regimes. This suggests that chloroplast translation may be affected differently by the relative availability of phosphorus and nitrogen than is cytoplasmic translation.

\section{Metabolic adaption to an imbalanced nutrient regime}

A significant portion of the proteins that were differentially expressed between nutrient regimes were involved in metabolism (see Figures 3.13, 3.15). Under an imbalanced nutrient regime, there was an increased abundance of proteins involved in carbohydrate metabolism, such as enzymes involved in both the synthesis and breakdown of glucose, in addition to carbohydrate transport. There was also an upregulation of proteins that mediate the TCA cycle and glycolysis, which are both energy-generation pathways. Energy is generated from glycolysis via the breakdown of glucose into pyruvate, and the TCA cycle by the catabolism of carbohydrates, fats and proteins. Previous transcriptomic and proteomic studies have observed an upregulation of glycolysis and the TCA cycle in response to low phosphorus availability in both higher plants (Li et al. 2007, Li et al. 2008, Zhang et al. 2014) and diatoms (Brembu et al. 2017). This is thought to be an adaptive mechanism to combat the decline in levels of ATP/ADP under phosphorus limitation (Duff et al. 1989, Plaxton and Podestá 2006). The metabolic shift towards energy generation under the imbalanced nutrient regime may be to counteract possible phosphorus limitation. Despite these changes in metabolism, the photosynthesis to respiration ratio of cultures under an imbalanced nutrient regime was the same as for cultures under the ambient regime, and higher than for cultures under an enriched regime at the control temperature, indicating the maintenance of metabolic balance and acclimation to altered nutrient availability.

Nutrient limitation and an imbalanced nutrient regime have been found to cause the accumulation of starch granules and lipid bodies in Symbiodiniaceae (Rosset et al. 2017). If required, these storage products can be broken down to provide a source of carbon and energy (Vitova et al. 2015) (see Figure 3.14). There was an increased abundance of proteins linked to starch and lipid droplet 
metabolism under an imbalanced nutrient regime. This included two proteins central to peroxisomal fatty acid Beta -oxidation. Fatty acid Beta -oxidation is the process by which fatty acids are broken down to generate $\mathrm{NADH}, \mathrm{FADH}_{2}$ and acetyl CoA, which is fed into the TCA cycle (Houten and Wanders 2010) and is linked to the peroxisomal breakdown of lipid droplets (Huang 2018). There was also an increased abundance of chloroplastic alpha-glucan water dikinase, which is integral to starch breakdown (Yu et al. 2001, Baunsgaard et al. 2005), and cytoplasmic phosphoglucomutase, which is linked to the breakdown and synthesis of starch (Malinova et al. 2014). The increased abundance of proteins tied to storage-product turn-over, suggests a possible mechanism by which Symbiodiniaceae may be combatting the stress of an imbalanced nutrient regime.

Reduced orthophosphate and ADP availability in phosphorus deficient autotrophs affects the function of metabolic pathways that require phosphate to transfer energy, namely glycolysis and respiratory electron transport (Plaxton and Tran 2011). A conserved adaptive mechanism in plants to counteract this is metabolic redundancy, whereby alternate enzymes can mediate a single reaction (Plaxton and Tran 2011, Liang et al. 2013). Under imbalanced nutrient conditions, there was an increased abundance of pyrophosphate--fructose 6-phosphate 1-phosphotransferase (PFP) subunit alpha 1, which is a regulatory unit that activates PFP (Lim et al. 2009). PFP can replace ATP-dependent phosphofructokinases, playing a central role in glycolysis by catalysing the interconversion of fructose-6-phosphate to fructose-1,6-bisphosphate (Ronimus et al. 1999). This enzyme uses pyrophosphate as a phosphoryl donor instead of ATP (Stitt 1990, Plaxton 1996), conserving energy and promoting the recycling of inorganic phosphorus, as inorganic phosphorus is a by-product of the reaction (Plaxton and Podestá 2006, Plaxton and Tran 2011). While not energy-conserving, alternative oxidase (AOX) can replace mitochondrial cytochrome $c$ oxidase as the terminal electron acceptor of mitochondrial electron transport in plants and Symbiodiniaceae (Rasmusson et al. 1990, Oakley et al. 2014a). Increased AOX activity has been observed in response to phosphorus limitation in both bean and tobacco plants (Rychter and Mikulska 1990, Sieger et al. 2005), and is thought to maintain respiratory electron flow when phosphorus deficiency limits oxidative phosphorylation. In this study ubiquinol oxidase 1a, mitochondrial AOX1 had an increased abundance under an imbalanced nutrient regime relative to the ambient and enriched regimes; however, this difference was only statistically significant for the ambient and imbalanced comparison. The increased abundance of PFP subunit $a$ and AOX under the imbalanced nutrient condition highlights the metabolic flexibility of Symbiodiniaceae and may represent metabolic adaption to phosphorus deficiency driven by imbalanced nitrogen to phosphorus levels. 


\section{Redox homeostasis}

Surprisingly, more proteins involved in redox homeostasis and the response to oxidative stress were differentially abundant between the three nutrient regimes than between the control and high temperatures $\left(25\right.$ and $34^{\circ} \mathrm{C}$ ) (see table $\mathrm{S} 1$ versus $\mathrm{S} 2$ and $\mathrm{S} 3$, appendix section $\mathrm{A} 2.2$ ). Compared to the ambient and enriched regimes, under an imbalanced nutrient regime there was both a decrease and increase in the abundance of several proteins that act as antioxidants and neutralise reactive species. Like other abiotic stressors, nutrient deficiency has been found to elevate levels of ROS and antioxidants in higher plants (Shin et al. 2005, Tewari et al. 2007, Torabi et al. 2009, Zhang et al. 2014). Although not demonstrated previously in Symbiodininaceae or dinoflagellates more generally, the significant change in the abundance of antioxidant proteins suggests that the antioxidant network is altered in response to nutrient stress. Due to the integral role that antioxidants play in the thermal stress tolerance of Symbiodiniaceae (Krueger et al. 2014, Hawkins et al. 2015), this link is worth further investigation, as it may contribute to the observed reduced thermal tolerance of Symbiodiniaceae under imbalanced nutrient conditions (Wiedenmann et al. 2013, Rosset et al. 2017).

\section{The conserved response of altered phospholipid metabolism in response to phosphorus deficiency}

Phosphorus is a major component of membranes in the form of phospholipids. In response to phosphorus limitation, a conserved response among cyanobacteria and eukaryotes is the replacement of phospholipids with non-phospholipids such as sulpholipids (Van Mooy et al. 2009). This response has also been observed in Symbiodiniaceae under an imbalanced nutrient regime (Wiedenmann et al. 2013) and is hypothesised to underlie the reduced thermal tolerance of Symbiodiniaceae under high nitrogen to phosphorus ratios (Wiedenmann et al. 2013, Rosset et al. 2017). Consistent with this, under an imbalanced nutrient regime, there was an upregulation of proteins involved in the fatty acid Beta -oxidation pathway (described above), which is implicated in the metabolism of membrane lipids (Cornah and Smith 2002). There was also an increased abundance of an uncharacterised sulfatase (PB10D8.02c), which is functionally annotated in the Uniprot kb database as being involved in sulfolipid metabolism; however, this is not substantiated with supporting literature (see tables S2 and S3, appendix section A2.2). The lack of clear evidence in the proteomics data that Symbiodiniaceae are altering membrane lipid composition in response to nutrient availability may reflect the relatively low abundance of enzymes involved in membrane lipid metabolism compared to other proteins detected in the proteome. 


\subsubsection{Conclusion}

The concept that motivated this study was that the adverse effects of eutrophication on the coral holobiont are driven not by the addition of nutrients, but by imbalanced nitrogen to phosphorus ratios causing phosphate deficiency in Symbiodiniaceae (Wiedenmann et al. 2013, D'Angelo and Wiedenmann 2014, Rosset et al. 2017). Specifically, this nutrient imbalance has been observed to decrease the photochemical efficiency of Symbiodiniaceae in hospite and increase bleaching susceptibility relative to enrichment with both nitrogen and phosphorus, or enrichment in favour of phosphorus (Wiedenmann et al. 2013, Rosset et al. 2017). This link between thermal tolerance and nutrient availability was not observed in this study, possibly due to the less extreme nitrogen to phosphorus ratio used for the imbalanced nutrient treatment (59:1 versus 211:1, Rosset et al., 2017), the different experimental species of Symbiodiniaceae, or because this experiment was performed in culture rather than in hospite. However, this study did provide evidence of phosphorus deficiency under an imbalanced nutrient regime. Previous studies have hypothesised that this phosphorus limitation is driven by high levels of nitrogen increasing symbiont population sizes (Wiedenmann et al. 2013, D'Angelo and Wiedenmann 2014). However, in this study, cultures under an imbalanced nutrient regime had severely depressed growth, even though the phosphorus levels in the enriched and imbalanced nutrient regimes were very similar, with the only difference being the relative availability of nitrogen. This evidence suggests that another mechanism other than increased growth rate may be mediating observed phosphorus limitation of Symbiodiniaceae under imbalanced nutrient regimes, highlighting an area for future research.

This study is the first to apply modern proteomics technology to Symbiodiniaceae under different temperature and nutrient treatments, providing novel insight into the shifts in cellular processes that underlie the relatively well characterised physiological responses to temperature and nutrients. Under thermal stress there was a down-regulation of proteins involved in energy-producing pathways (glycolysis, the TCA cycle, and the Calvin cycle), as well as energy-consuming pathways (protein synthesis and fatty acid synthesis), which has not been observed in Symbiodiniaceae before. Also, while photosynthetic protein expression has been found to alter in response to thermal stress (Gierz et al. 2017), the consistent down-regulation of all identified proteins observed here is unique. Overall, this could simply reflect a breakdown of cellular function, as photosynthesis to respiration ratios were negative at $34^{\circ} \mathrm{C}$, indicating that the $B$. minutum cells were experiencing lethal thermal stress. Interestingly, more proteins involved in redox homeostasis were differentially abundant between the three nutrient regimes than between the control and high temperatures $\left(25\right.$ and $\left.34^{\circ} \mathrm{C}\right)$. This link 
between the antioxidant network and nutrient availability is novel and should be a target for future research.

The insight into cellular functioning provided by this study is particularly significant in the case of nutrient availability, as this is the first example of 'omics' techniques applied to Symbiodiniacae under different nutrient regimes. The proteome of cultures under ambient and enriched treatments were similar, while the proteome under the imbalanced nutrient treatment was divergent from both of these treatments. This trend demonstrates that the relative availability of nitrogen to phosphorus determines the response of Sybiodiniaceae cellular physiology to the nutrient environment, highlighting the importance of considering nutrient stoichiometry in future nutrient enrichment studies of both corals and their endosymbionts. Despite protein abundance indicating multiple shifts in protein and carbohydrate metabolism under the imbalanced nutrient regime (see Figure 3.14), physiological measurements of photosynthetic efficiency and oxygen flux indicated B. minutum was able to adapt its physiology to maintain cellular homeostasis, despite showing signs of phosphorus deficiency. Future research should determine whether this physiological adaptability is maintained in hospite. This study demonstrates how proteomics technology can be applied to further the understanding of the cellular mechanisms underlying the physiological response of Symbiodiniacae to its environment. Notably, this analysis is constrained by the limitations of protein homology, and many dinoflagellate proteins are uncharacterised (24\% of those identified in this study), which restricts the inferences that can be made by this data set. However, as the field of dinoflagellate proteomics grows, this data set could be reanalysed. 


\section{Chapter 4: General Discussion}

This thesis explores the physiological response of Breviolum minutum to both elevated temperature and different nutrient environments. Chapter 2 demonstrates intraspecies variability in thermal tolerance within $B$. minutum, emphasised by the difference in physiological responses to thermal stress, depending on whether the temperature is slowly or rapidly increased. To further understand the cellular mechanisms underlying these physiological responses to environmental stress, the third chapter utilises novel proteomics technology to explore the proteomic response of B. minutum to different temperature and nutrient treatments. Even though no interaction between temperature and nutrient regime was found, individually both temperature and nutrient regime had a significant impact on protein abundance. At elevated temperature there was upregulation of proteins that act to stabilise protein structure and maintain cellular redox homeostasis; however, there was a reduction in photosynthesis proteins, ribosomal proteins, and metabolic proteins (Calvin cycle/glycolysis). These findings together with respirometry measurements indicating a negative metabolic balance, indicates B. minutum exhibited a shut-down in cellular function in response to lethal thermal stress.

The physiological response to nutrient availability was less dramatic, however, proteomic data highlighted significant changes occurring at a cellular level. The proteome under ambient nutrient levels and enrichment with both nitrogen and phosphorus had negligible differences, however, the proteome under an imbalanced nutrient regime (i.e. high nitrogen, ambient phosphorus levels) was significantly different. Differential protein abundance suggests that under imbalanced nutrient levels there was a reduction in carbon fixation, with a concomitant increase in protein translation and metabolic pathways involved in energy generation and storage-product turn-over. However, despite these alterations to cellular functioning under an imbalanced nutrient regime, the photosynthetic efficiency and metabolic balance (i.e. P:R ratio) was not lower relative to measurements under the ambient or enriched regime, indicating metabolic adaption to maintain cellular function. In addition, under the imbalanced nutrient regime inhibited population growth, increased alkaline phosphatase activity, and an upregulation of the tricarboxylic acid (TCA) cycle and glycolysis was observed, suggesting that the B. minutum cultures under an imbalanced nutrient regime were experiencing phosphate deficiency, as has been demonstrated previously (Wiedenmann et al. 2013, Rosset et al. 2017).

The results of both chapters pose questions for future research. The results of chapter two provoke the question of how differential thermal tolerance between two strains of the same species of Symbiodiniacae may provide an adaptive advantage to the coral holobiont. Chapter three represents 
the first example of 'omics' techniques applied to Symbiodiniaceae under different levels of nutrient availability, highlighting the dramatic influence of this factor on cellular metabolic processes, and leading to the question of how 'omics' technology can be used to direct future research in order to further our understanding of the effects eutrophication on the coral holobiont. The following sections address these questions, followed by a final section which considers the results of this thesis in the context of knowledge-based reef management.

\subsection{How might the barrier of host-symbiont specificity be overcome in the context of adaptive bleaching?}

Coral bleaching has been proposed as an adaptive mechanism (Buddemeier and Fautin 1993), allowing corals to take up new more thermally tolerant symbionts from the surrounding environment (switching), or permitting a background resident symbiont type to proliferate and become the dominant (shuffling) (Baker 2001, 2003). However, this hypothesis has been a topic of controversy (Hoegh-Guldberg et al. 2002, Douglas 2003, Stat and Gates 2011). There is evidence for both switching and shuffling (Baker 2001, Lewis and Coffroth 2004, Cunning et al. 2015, Silverstein et al. 2015, Bay et al. 2016), but it has also been demonstrated that the novel or altered symbiosis is not sustained and is ephemeral (Thornhill et al. 2006, Coffroth et al. 2010, Stat and Gates 2011, Hill et al. 2014). This may be because newly obtained symbionts are not necessarily beneficial, causing poor symbiosis function and stress (Stat and Gates 2011, Cunning et al. 2015, Matthews et al. 2017).

Underpinning 'the adaptive bleaching hypothesis' is the concept that the cnidarian-dinoflagellate symbiosis can be flexible. However, the proposition of symbiont flexibility may be misguided. Supporting this, a global meta-analysis of coral-Symbiodiniaceae combinations demonstrates that the majority of coral species are specialists (Fabina et al. 2012). In alignment, analysis of the genetic structure of coral populations in the Hawaiian Islands indicate that these associations are consistent with a model of co-evolution (Stat et al. 2015), thus demonstrating a highly conserved and specific symbioses. Many long-term studies have illustrated stability in symbiont composition despite environmental stressors (Goulet and Coffroth 2003, Thornhill et al. 2006, LaJeunesse et al. 2010, McGinley et al. 2012a). Thus, the switching or shuffling of symbionts in response to environmental stress may be restricted to a few coral species. Many corals can only associate with a taxonomicallynarrow complement of symbiotic partners. This restriction could be a caused by host genetics (Barshis et al. 2010), or the specificity of symbioses with regards to host-symbiont recognition molecules, which mediate the establishment and maintenance of the symbiosis (Davy et al. 2012). 
However, the limitations of host-symbiont specificity may be overcome if a coral were to establish a symbiosis with a more thermally resistant partner of the same species. To this end, the second chapter of this thesis provides evidence for differential thermal tolerance between two strains of Breviolum minutum. The NZ01 strain was able to maintain photosynthetic function and metabolic balance up to $34^{\circ} \mathrm{C}$, while in concert the FlAp2 strain exhibited severe photoinhibition and a negative metabolic balance. Future research should aim to test if the thermal tolerance of NZ01 could confer greater symbiosis stability and bleaching resistance in hospite compared to FlAp2. Variability in intraspecies thermal tolerance lends a new perspective to the 'adaptive bleaching hypothesis', as post thermally induced bleaching a more resistant strain of the same species could repopulate a coral, conferring an adaptive advantage. To this end, multiple symbiont genotypes have been identified within one coral host (Parkinson et al. 2015). Due to their fast generation times (Wilkerson et al. 1988), large population sizes (Drew 1972, Littman et al. 2008) and high mutation rates (Van Oppen et al. 2011), Symbiodiniaceae species have the potential to accumulate genetic variation in the form of new genotypes. As coral reefs face rising sea surface temperatures due to global warming, population shifts within the holobiont to more thermally resistant strains of the same species in conjunction with natural selection on symbiont genotypes may provide hope for the adaption and survival of coral reefs into an uncertain future.

\subsection{How might 'omics' technology elucidate the impact of eutrophication on coral reefs at a cellular level and highlight potential avenues for future research?}

Anthropogenic presence along the coastline has a dramatic influence on coastal ecosystems as the natural environment is altered and shaped to fit our needs. A significant effect of this anthropogenic activity is the input of nutrients into coastal ecosystems. This issue is expected to worsen as global warming is projected to increase rainfall variability, resulting in the increased frequency of flooding, and thus nutrient and sediment laden freshwater entering coastal systems (Brodie et al. 2010, Wagner et al. 2010). An ecosystem particularly vulnerable to eutrophication is coral reefs, which usually thrive in oligotrophic tropical waters (Odum and Odum 1955). Nutrient-enriched waters have been linked to the degradation of coral reef ecosystems and a shift to macroalgal dominance (De'ath and Fabricius 2010, Fabricius et al. 2012, Wooldridge 2016).

Eutrophication can reduce the stability of the symbiosis between corals and dinoflagellate algae, which underlies the success of this ecosystem. Increased nutrient availability can cause enlarged symbiont populations which become a carbon sink instead of a carbon source (Ezzat et al. 2015, 
Wooldridge 2016). Consequently, corals in nutrient-enriched waters have been observed to exhibit reduced tissue thickness (McGuire and Szmant 1997, Cruz-Pinon et al. 2003), gamete production (Tomascik and Sander 1987, Loya et al. 2004), and skeletal growth rates (Marubini and Davies 1996, Ferrier-Pagès et al. 2001). Furthermore, the relative availability of nitrogen to phosphorus is essential for determining the response of corals and their symbionts to eutrophication. Nutrient enrichment has adverse effects on Symbiodiniaceae stress tolerance, paradoxically via imbalanced nutrient ratios causing phosphorus deficiency, resulting in photosynthetic dysfunction, reduced carbon fixation, and a lower thermal bleaching tolerance of the holobiont (Wiedenmann et al. 2013, Ezzat et al. 2015, Rosset et al. 2017). The physiological effects of eutrophication on corals and their endosymbionts are relatively well characterised (Fabricius 2005, D'Angelo and Wiedenmann 2014). However, there is a lack of understanding of the effect of nutrient availability on cellular processes and functioning.

The insight into the effects of nutrient availability on cellular function provided by 'omics' data can highlight potential avenues for future research. This thesis represents the first example of an 'omics' technique being applied to Symbiodiniaceae cultures exposed to different nutrient regimes, providing novel insight into how cellular function is altered by nutrient availability. The proteomics data highlighted that nutrient availability had a dramatic influence on the abundance of enzymes involved in redox homeostasis. It is generally accepted that the build-up of reactive oxygen species (ROS) and the resultant oxidative stress, sparks the cellular cascade of cnidarian bleaching (Weis 2008, Rehman et al. 2016, Oakley and Davy 2018). ROS can oxidise membranes, denature proteins and damage nucleic acids, causing significant structural and functional cellular damage in both the symbiont and host (Lesser 2006). Both partners produce antioxidant enzymes which neutralise these reactive species, such as ascorbate peroxidase (APX), catalase and different forms of superoxide dismutase (SOD) (Richier et al. 2005, Lesser 2006, Merle et al. 2007). The antioxidant network of Symbiodiniaceae has a dramatic influence on species specific thermal sensitivity (Krueger et al. 2014, Hawkins et al. 2015). Thus, the altered abundance of antioxidant enzymes in response to different nutrient treatments observed in this study warrants further investigation as this link may further explain the effect of nutrient availability on Symbiodiniaceae thermal tolerance and subsequently host bleaching susceptibility.

In this study different nutrient treatments had a minimal effect on measurements of photosynthetic and metabolic health, however, the proteomics data revealed that on a cellular level there were dramatic shifts in reaction pathways in response to an imbalanced nutrient regime. Specifically, there was an upregulation of carbohydrate metabolism, energy generation pathways such as the TCA cycle and glycolysis, and enzymes involved in storage product turn over (starch/lipid). In addition, there 
was strong evidence of increased protein production, presumably to mediate this upregulated cellular work. Together the proteomic and physiological data collected in this study suggests that by altering carbohydrate metabolism, utilising storage products and halting cellular growth, B. minutum was able to maintain photosynthetic capacity and cellular homeostasis. However, it is possible that in symbiosis $B$. minutum may not be able to maintain physiological function under an imbalanced nitrogen to phosphorus ratio, as in hospite the majority of photosynthates are transferred to the host (Muscatine et al. 1984), leaving less resources to make the necessary alterations to metabolism. This may explain why in contrast to this study, other studies that have investigated the impact of an imbalanced nutrient ratio on Symbiodiniaceae in hospite have observed reduced photosynthetic capacity, carbon fixation and thermal tolerance of Symbiodiniaceae (Wiedenmann et al. 2013, Ezzat et al. 2015, Rosset et al. 2017).

Future work should define how Symbiodiniaceae metabolism is altered in response to the relative availability of nitrogen to phosphorus both in hospite and in culture. To this end a comparative metabolomics study would provide detailed insight into the response of metabolic pathways to nutrient availability, and how these responses may change in symbiosis. Gaining this knowledge is important as metabolic exchange underpins the symbiosis between cnidarian corals and Symbiodiniaceae, and any alteration in Symbiodiniaceae metabolism may influence symbiosis stability (Wooldridge 2013, Matthews et al. 2017). Due to the impact of eutrophication on the thermal tolerance of corals (Rosset et al. 2017), as well as the nutrient ecology of this delicate symbiosis (Ezzat et al. 2015), it is essential to understand how this stressor may impact coral reef survival fully. 'Omics' technology is an invaluable tool to reveal the metabolic changes on a cellular level that underlie observed physiological responses, and direct avenues of research that will facilitate knowledge-based management of coral reefs.

\subsection{The results of this thesis in the context of knowledge-based reef management}

While the threats faced by coral reefs due to global warming remain the hardest to counter, effective coral reef management can build coral reef resilience (West and Salm 2003; Carilli et al. 2009; D'Angelo and Wiedenmann 2014). Underpinning the success of coral reef management is knowledge, as an accurate understanding of an ecosystem is required to guide management action.

The intraspecies variability in thermal tolerance demonstrated in this thesis highlights the intraspecies physiological plasticity of the Symbiodiniaceae family. Compared to interspecies diversity, the intraspecies diversity of Symbiodiniaceae is poorly characterised (Swain et al. 2017). Future 
research should prioritise population genetic studies in order to characterise this genetic diversity (Palstra and Ruzzante 2008), along with the connectivity between populations (Palumbi 2003), and how these populations change with time and the environment (Van Oppen and Gates 2006). All these factors can determine coral reef resilience, thus, integrating a spatial representation of genetic diversity into management models would increase the accuracy of the prediction of coral bleaching events (Palumbi 2003, Van Oppen and Gates 2006). In addition, tracking how the diversity of populations change over time would provide insight into how genetic diversity influences recovery after bleaching events, and determine if connectivity between symbiont populations allows the spread of more thermally resistant genotypes, facilitating the evolutionary adaption of the coral holobiont (Van Oppen and Gates 2006, Howells et al. 2009).

The proteomic results of this study demonstrated that the effect of nutrient levels on Symbiodiniaceae cellular function was determined by the nitrogen to phosphorus ratio. Currently, thresholds for nutrient input into coastal ecosystems are primarily based on achievability instead of ecological impact (Brodie et al. 2017b). The relative abundance of nitrogen to phosphorus is not considered, despite the importance of nutrient stoichiometry in determining the physiological response of the coral holobiont to the nutrient environment (D'Angelo and Wiedenmann 2014). Partially, this is because the exact threshold of nitrogen to phosphorus that has a negative influence on the holobiont and its resistance to thermal stress is not known. This knowledge gap could be addressed by applying 'omics' methods in conjunction with physiological measurements to reef corals both in areas of different nutrient levels in the field, and in manipulative experiments. Both manipulative and modelling studies suggest that reducing eutrophication of coastal ecosystems may build the reliance of coral reefs to global warming (Thurber et al. 2014, Wooldridge 2016). Thus, gaining the knowledge required to set ecologically relevant nutrient input limits is essential to safeguarding coral reefs and the biodiversity they support into the future. 


\section{References}

Abrego, D., K. E. Ulstrup, B. L. Willis, and M. J. van Oppen. 2008. Species-specific interactions between algal endosymbionts and coral hosts define their bleaching response to heat and light stress. Proceedings of the Royal Society B: Biological Sciences 275:2273-2282.

Aihara, Y., S. Takahashi, and J. Minagawa. 2016. Heat induction of cyclic electron flow around photosystem I in the symbiotic dinoflagellate Symbiodinium. Plant Physiology:pp. 01886.02015.

Ainsworth, T. D., S. F. Heron, J. C. Ortiz, P. J. Mumby, A. Grech, D. Ogawa, C. M. Eakin, and W. Leggat. 2016. Climate change disables coral bleaching protection on the Great Barrier Reef. Science 352:338-342.

Anderson, S., and J. Burris. 1987. Role of glutamine synthetase in ammonia assimilation by symbiotic marine dinoflagellates (zooxanthellae). Marine Biology 94:451-458.

Annis, E. R., and C. B. Cook. 2002. Alkaline phosphatase activity in symbiotic dinoflagellates (zooxanthellae) as a biological indicator of environmental phosphate exposure. Marine Ecology Progress Series 245:11-20.

Asada, K. 1999. The water-water cycle in chloroplasts: scavenging of active oxygens and dissipation of excess photons. Annual Review of Plant Biology 50:601-639.

Bachvaroff, T. R., and A. R. Place. 2008. From stop to start: tandem gene arrangement, copy number and trans-splicing sites in the dinoflagellate Amphidinium carterae. PLoS ONE 3:e2929.

Badger, M. R., T. J. Andrews, S. Whitney, M. Ludwig, D. C. Yellowlees, W. Leggat, and G. D. Price. 1998. The diversity and coevolution of Rubisco, plastids, pyrenoids, and chloroplastbased CO2-concentrating mechanisms in algae. Canadian Journal of Botany 76:1052-1071.

Badger, M. R., S. von Caemmerer, S. Ruuska, and H. Nakano. 2000. Electron flow to oxygen in higher plants and algae: rates and control of direct photoreduction (Mehler reaction) and rubisco oxygenase. Philosophical Transactions of the Royal Society of London B: Biological Sciences 355:1433-1446.

Baghdasarian, G., and L. Muscatine. 2000. Preferential expulsion of dividing algal cells as a mechanism for regulating algal-cnidarian symbiosis. The Biological Bulletin 199:278-286.

Baird, A. H., R. Bhagooli, P. J. Ralph, and S. Takahashi. 2009. Coral bleaching: the role of the host. Trends in Ecology \& Evolution 24:16-20.

Baker, A. C. 2001. Ecosystems: reef corals bleach to survive change. Nature 411:765.

Baker, A. C. 2003. Flexibility and specificity in coral-algal symbiosis: diversity, ecology, and biogeography of Symbiodinium. Annual Review of Ecology, Evolution, and Systematics 34:661-689.

Baker, A. C., P. W. Glynn, and B. Riegl. 2008. Climate change and coral reef bleaching: An ecological assessment of long-term impacts, recovery trends and future outlook. Estuarine, Coastal and Shelf Science 80:435-471.

Baker, D. M., J. P. Andras, A. G. Jordán-Garza, and M. L. J. T. I. j. Fogel. 2013. Nitrate competition in a coral symbiosis varies with temperature among Symbiodinium clades. The ISME Jornal 7:1248.

Barlow, S., and R. Triemer. 1986. Phosphatase localization in the endomembrane system of the dinoflagellate Crypthecodinium cohnii. Journal of Histochemistry \& Cytochemistry 34:10211027.

Barott, K. L., A. A. Venn, S. O. Perez, S. Tambutte, and M. Tresguerres. 2015a. Coral host cells acidify symbiotic algal microenvironment to promote photosynthesis. Proceedings of the National Academy of Sciences of the United States of America 112:607-612.

Barott, K. L., A. A. Venn, S. O. Perez, S. Tambutté, and M. Tresguerres. 2015b. Coral host cells acidify symbiotic algal microenvironment to promote photosynthesis. Proceedings of the National Academy of Sciences 112:607-612. 
Barshis, D. J., J. T. Ladner, T. A. Oliver, and S. R. Palumbi. 2014. Lineage-specific transcriptional profiles of Symbiodinium spp. unaltered by heat stress in a coral host. Molecular Biology and Evolution 31:1343-1352.

Barshis, D. J., J. H. Stillman, R. D. Gates, R. J. Toonen, L. W. Smith, and C. Birkeland. 2010. Protein expression and genetic structure of the coral Porites lobata in an environmentally extreme Samoan back reef: does host genotype limit phenotypic plasticity? Molecular Ecology 19:1705-1720.

Baskett, M. L., S. D. Gaines, and R. M. Nisbet. 2009. Symbiont diversity may help coral reefs survive moderate climate change. Ecological Applications 19:3-17.

Baunsgaard, L., H. Lütken, R. Mikkelsen, M. A. Glaring, T. T. Pham, and A. Blennow. 2005. A novel isoform of glucan, water dikinase phosphorylates pre-phosphorylated $\alpha$-glucans and is involved in starch degradation in Arabidopsis. The Plant Journal 41:595-605.

Bay, L. K., V. R. Cumbo, D. Abrego, J. T. Kool, T. D. Ainsworth, and B. L. Willis. 2011. Infection dynamics vary between Symbiodinium types and cell surface treatments during establishment of endosymbiosis with coral larvae. Diversity 3:356-374.

Bay, L. K., J. Doyle, M. Logan, and R. Berkelmans. 2016. Recovery from bleaching is mediated by threshold densities of background thermo-tolerant symbiont types in a reef-building coral. Royal Society Open Science 3:160322.

Bayer, T., M. Aranda, S. Sunagawa, L. K. Yum, M. K. DeSalvo, E. Lindquist, M. A. Coffroth, C. R. Voolstra, and M. Medina. 2012. Symbiodinium transcriptomes: genome insights into the dinoflagellate symbionts of reef-building corals. PLoS ONE 7:e35269.

Bayliss, S. L., Z. R. Scott, M. A. Coffroth, and C. P. terHorst. Genetic variation in Breviolum antillogorgium, a coral reef symbiont, in response to temperature and nutrients. Ecology and Evolution.

Béraud, E., F. Gevaert, C. Rottier, and C. Ferrier-Pagès. 2013. The response of the scleractinian coral Turbinaria reniformis to thermal stress depends on the nitrogen status of the coral holobiont. Journal of Experimental Biology 216:2665-2674.

Berkelmans, R., and M. J. Van Oppen. 2006. The role of zooxanthellae in the thermal tolerance of corals: a 'nugget of hope'for coral reefs in an era of climate change. Proceedings of the Royal Society of London B: Biological Sciences 273:2305-2312.

Berkson, J., T. B. Magath, and M. Hurn. 1939. The error of estimate of the blood cell count as made with the hemocytometer. American Journal of Physiology-Legacy Content 128:309-323.

Berry, J., and O. Bjorkman. 1980. Photosynthetic response and adaptation to temperature in higher plants. Annual Review of Plant Physiology 31:491-543.

Bertucci, A., A. Moya, S. Tambutté, D. Allemand, C. T. Supuran, and D. Zoccola. 2013. Carbonic anhydrases in anthozoan corals-A review. Bioorganic \& Medicinal Chemistry 21:14371450.

Bhagooli, R. 2013. Inhibition of Calvin-Benson cycle suppresses the repair of photosystem II in Symbiodinium: implications for coral bleaching. Hydrobiologia 714:183-190.

Bhagooli, R., and M. Hidaka. 2003. Comparison of stress susceptibility of in hospite and isolated zooxanthellae among five coral species. Journal of Experimental Marine Biology and Ecology 291:181-197.

Björkman, K., and D. M. Karl. 1994. Bioavailability of inorganic and organic phosphorus compounds to natural assemblages of microorganisms in Hawaiian coastal waters. Marine Ecology Progress Series:265-273.

Bonnefond, L., A. Fender, J. Rudinger-Thirion, R. Giegé, C. Florentz, and M. Sissler. 2005. Toward the full set of human mitochondrial aminoacyl-tRNA synthetases: characterization of AspRS and TyrRS. Biochemistry 44:4805-4816.

Boston, R. S., P. V. Viitanen, and E. Vierling. 1996. Molecular chaperones and protein folding in plants. Post-transcriptional control of gene expression in plants. Springer 191-222

Bothe, H., S. Ferguson, and W. E. Newton. 2006. Biology of the nitrogen cycle. Elsevier. 
Bouchard, J. N., and H. Yamasaki. 2008. Heat stress stimulates nitric oxide production in Symbiodinium microadriaticum: a possible linkage between nitric oxide and the coral bleaching phenomenon. Plant and Cell Physiology 49:641-652.

Brembu, T., A. Mühlroth, L. Alipanah, and A. M. Bones. 2017. The effects of phosphorus limitation on carbon metabolism in diatoms. Philosophical Transactions of the Royal Society B: Biological Sciences 372:20160406.

Brodie, J., M. Devlin, and S. Lewis. 2017a. Potential enhanced survivorship of crown of thorns starfish larvae due to near-annual nutrient enrichment during secondary outbreaks on the central mid-shelf of the Great Barrier Reef, Australia. Diversity 9:17.

Brodie, J., F. Kroon, B. Schaffelke, E. Wolanski, S. Lewis, M. Devlin, I. Bohnet, Z. Bainbridge, J. Waterhouse, and A. Davis. 2012. Terrestrial pollutant runoff to the Great Barrier Reef: an update of issues, priorities and management responses. Marine Pollution Bulletin 65:81-100.

Brodie, J., T. Schroeder, K. Rohde, J. Faithful, B. Masters, A. Dekker, V. Brando, and M. Maughan. 2010. Dispersal of suspended sediments and nutrients in the Great Barrier Reef lagoon during river-discharge events: conclusions from satellite remote sensing and concurrent flood-plume sampling. Marine and Freshwater Research 61:651-664.

Brodie, J. E., S. E. Lewis, C. J. Collier, S. Wooldridge, Z. T. Bainbridge, J. Waterhouse, M. A. Rasheed, C. Honchin, G. Holmes, and K. Fabricius. 2017b. Setting ecologically relevant targets for river pollutant loads to meet marine water quality requirements for the Great Barrier Reef, Australia: A preliminary methodology and analysis. Ocean \& Coastal Management 143:136-147.

Brown, B., I. Ambarsari, M. Warner, W. Fitt, R. Dunne, S. Gibb, and D. Cummings. 1999. Diurnal changes in photochemical efficiency and xanthophyll concentrations in shallow water reef corals: evidence for photoinhibition and photoprotection. Coral Reefs 18:99-105.

Brown, B. E. 1997. Coral bleaching: causes and consequences. Coral Reefs 16:S129-S138.

Bruce, T. J., M. C. Matthes, J. A. Napier, and J. A. Pickett. 2007. Stressful "memories" of plants: evidence and possible mechanisms. Plant Science 173:603-608.

Buchfink, B., C. Xie, and D. H. Huson. 2015. Fast and sensitive protein alignment using DIAMOND. Nature methods 12:59.

Buddemeier, R. W., and D. G. Fautin. 1993. Coral bleaching as an adaptive mechanism. Bioscience 43:320-326.

Buxton, L., M. Badger, and P. Ralph. 2009. Effects of moderate heat stress and dissolved inorganic carbon concentration on photosynthesis and respiration of symbiodinium sp.(dinophyceae) in culture and in symbiosis. Journal of Phycology 45:357-365.

Cantin, N. E., M. J. van Oppen, B. L. Willis, J. C. Mieog, and A. P. J. C. R. Negri. 2009. Juvenile corals can acquire more carbon from high-performance algal symbionts. 28:405.

Cardol, P., B. Bailleul, F. Rappaport, E. Derelle, D. Béal, C. Breyton, S. Bailey, F. A. Wollman, A. Grossman, and H. Moreau. 2008. An original adaptation of photosynthesis in the marine green alga Ostreococcus. Proceedings of the National Academy of Sciences 105:7881-7886.

Cardol, P., G. Forti, and G. Finazzi. 2011. Regulation of electron transport in microalgae. Biochimica et Biophysica Acta (BBA)-Bioenergetics 1807:912-918.

Carilli, J., S. D. Donner, and A. C. Hartmann. 2012. Historical temperature variability affects coral response to heat stress. PLoS ONE 7:e34418.

Carlos, A. A., B. K. Baillie, M. Kawachi, and T. Maruyama. 1999. Phylogenetic position of Symbiodinium (Dinophyceae) isolates from tridacnids (Bivalvia), cardiids (Bivalvia), a sponge (Porifera), a soft coral (Anthozoa), and a free-living strain. Journal of Phycology 35:1054-1062.

Carpenter, S. R., N. F. Caraco, D. L. Correll, R. W. Howarth, A. N. Sharpley, and V. H. Smith. 1998. Nonpoint pollution of surface waters with phosphorus and nitrogen. Ecological Applications 8:559-568. 
Chakravarti, L. J., V. H. Beltran, and M. J. van Oppen. 2017. Rapid thermal adaptation in photosymbionts of reef-building corals. Global Change Biology 23:4675-4688.

Chen, M.-C., Y.-M. Cheng, M.-C. Hong, and L.-S. Fang. 2004. Molecular cloning of Rab5 (ApRab5) in Aiptasia pulchella and its retention in phagosomes harboring live zooxanthellae. Biochemical and Biophysical Research Communications 324:1024-1033.

Chen, M.-C., M.-C. Hong, Y.-S. Huang, M.-C. Liu, Y.-M. Cheng, and L.-S. Fang. 2005. ApRab11, a cnidarian homologue of the recycling regulatory protein Rab11, is involved in the establishment and maintenance of the Aiptasia-Symbiodinium endosymbiosis. Biochemical and Biophysical Research Communications 338:1607-1616.

Cinner, J. 2014. Coral reef livelihoods. Current Opinion in Environmental Sustainability 7:65-71.

Coffroth, M. A., D. M. Poland, E. L. Petrou, D. A. Brazeau, and J. C. Holmberg. 2010. Environmental symbiont acquisition may not be the solution to warming seas for reefbuilding corals. PLoS ONE 5:e13258.

Coffroth, M. A. J. P. 2005. Genetic diversity of symbiotic dinoflagellates in the genus Symbiodinium. Protist 156:19-34.

Coker, D. J., S. K. Wilson, and M. S. Pratchett. 2014. Importance of live coral habitat for reef fishes. Reviews in Fish Biology and Fisheries 24:89-126.

Coles, S. 1997. Reef corals occurring in a highly fluctuating temperature environment at Fahal Island, Gulf of Oman (Indian Ocean). Coral Reefs, 16: 269-272

Coles, S., and P. Jokiel. 1977. Effects of temperature on photosynthesis and respiration in hermatypic corals. Marine Biology 43:209-216.

Coles, S. L., and B. E. Brown. 2003. Coral bleaching-capacity for acclimatization and adaptation. Advances in Marine Biology 46:184-226.

Conley, D. J., H. W. Paerl, R. W. Howarth, D. F. Boesch, S. P. Seitzinger, K. E. Havens, C. Lancelot, and G. E. Likens. 2009. Controlling eutrophication: nitrogen and phosphorus. Science 323:1014-1015.

Cook, C., C. D'Elia, and G. Muller-Parker. 1988. Host feeding and nutrient sufficiency for zooxanthellae in the sea anemone Aiptasia pallida. Marine Biology 98:253-262.

Cook, C. B., and S. K. Davy. 2001. Are free amino acids responsible for thehost factor'effects on symbiotic zooxanthellae in extracts of host tissue? Hydrobiologia 461:71-78.

Cornah, J. E., and S. M. Smith. 2002. Synthesis and function of glyoxylate cycle enzymes. Pages 57101 Plant peroxisomes. Springer.

Costanza, R., R. d'Arge, R. De Groot, S. Farber, M. Grasso, B. Hannon, K. Limburg, S. Naeem, R. V. O'neill, and J. Paruelo. 1997. The value of the world's ecosystem services and natural capital. Nature 387:253.

Cox, J., M. Y. Hein, C. A. Luber, I. Paron, N. Nagaraj, and M. Mann. 2014. Accurate proteome-wide label-free quantification by delayed normalization and maximal peptide ratio extraction, termed MaxLFQ. Molecular \& Cellular Proteomics 13:2513-2526.

Cox, J., and M. Mann. 2008. MaxQuant enables high peptide identification rates, individualized ppbrange mass accuracies and proteome-wide protein quantification. Nature Biotechnology 26:1367.

Crawley, A., D. I. Kline, S. Dunn, K. Anthony, and S. Dove. 2010. The effect of ocean acidification on symbiont photorespiration and productivity in Acropora formosa. Global Change Biology 16:851-863.

Cruz-Pinon, G., J. Carricart-Ganivet, and J. Espinoza-Avalos. 2003. Monthly skeletal extension rates of the hermatypic corals Montastraea annularis and Montastraea faveolata: biological and environmental controls. Marine Biology 143:491-500.

Cunning, R., and A. C. Baker. 2013. Excess algal symbionts increase the susceptibility of reef corals to bleaching. Nature Climate Change 3:259. 
Cunning, R., N. Vaughan, P. Gillette, T. R. Capo, J. L. Mate, and A. C. Baker. 2015. Dynamic regulation of partner abundance mediates response of reef coral symbioses to environmental change. Ecology 96:1411-1420.

D'Angelo, C., and J. Wiedenmann. 2014. Impacts of nutrient enrichment on coral reefs: new perspectives and implications for coastal management and reef survival. Current Opinion in Environmental Sustainability 7:82-93.

D'elia, C., S. Domotor, and K. Webb. 1983. Nutrient uptake kinetics of freshly isolated zooxanthellae. Marine Biology 75:157-167.

D’Angelo, C., and J. Wiedenmann. 2014. Impacts of nutrient enrichment on coral reefs: new perspectives and implications for coastal management and reef survival. Current Opinion in Environmental Sustainability 7:82-93.

Da Silva Costa, O. 2001. Nutrification and its effects on coral reefs from Southern Bahia, Brazil. University of Plymouth.

Davies, P. S. 1991. Effect of daylight variations on the energy budgets of shallow-water corals. Marine Biology 108:137-144.

Davies, S. W., J. B. Ries, A. Marchetti, and K. D. Castillo. 2018. Symbiodinium functional diversity in the coral Siderastrea siderea is influenced by thermal stress and reef environment, but not ocean acidification. Frontiers in Marine Science 5:150.

Davison, I. R. 1991. Environmental effects on algal photosynthesis: temperature. Journal of Phycology 27:2-8.

Davy, S. K., D. Allemand, and V. M. Weis. 2012. Cell biology of cnidarian-dinoflagellate symbiosis. Microbiology and Molecular Biology Reviews 76:229-261.

De'ath, G., and K. Fabricius. 2010. Water quality as a regional driver of coral biodiversity and macroalgae on the Great Barrier Reef. Ecological Applications 20:840-850.

De'ath, G., J. M. Lough, and K. E. Fabricius. 2009. Declining coral calcification on the Great Barrier Reef. Science 323:116-119.

De Bary, A. 1879. Die erscheinung der symbiose, Strasbourg, Germany.

De'ath, G., K. E. Fabricius, H. Sweatman, and M. Puotinen. 2012. The 27-year decline of coral cover on the Great Barrier Reef and its causes. Proceedings of the National Academy of Sciences 109:17995-17999.

Del Pozo, J. C., I. Allona, V. Rubio, A. Leyva, A. De La Peña, C. Aragoncillo, and J. Paz-Ares. 1999. A type 5 acid phosphatase gene from Arabidopsis thaliana is induced by phosphate starvation and by some other types of phosphate mobilising/oxidative stress conditions. The Plant Journal 19:579-589.

Detournay, O., C. E. Schnitzler, A. Poole, and V. M. Weis. 2012. Regulation of cnidariandinoflagellate mutualisms: Evidence that activation of a host TGF beta innate immune pathway promotes tolerance of the symbiont. Developmental and Comparative Immunology 38:525-537.

DeVantier, L., G. De'Ath, E. Turak, T. Done, and K. Fabricius. 2006. Species richness and community structure of reef-building corals on the nearshore Great Barrier Reef. Coral Reefs 25:329-340.

Díaz-Almeyda, E., P. Thomé, M. El Hafidi, and R. Iglesias-Prieto. 2011. Differential stability of photosynthetic membranes and fatty acid composition at elevated temperature in Symbiodinium. Coral Reefs 30:217-225.

Donner, S. D., G. J. Rickbeil, and S. F. Heron. 2017. A new, high-resolution global mass coral bleaching database. PLoS ONE 12:e0175490.

Donner, S. D., W. J. Skirving, C. M. Little, M. Oppenheimer, and O. Hoegh-Guldberg. 2005. Global assessment of coral bleaching and required rates of adaptation under climate change. Global Change Biology 11:2251-2265.

Doonan, B. B., and T. E. Jensen. 1980. Ultrastructural localization of alkaline phosphatase in the cyanobacteriaCoccochloris peniocytis andAnabaena cylindrica. Protoplasma 102:189-197. 
Douglas, A. 2003. Coral bleaching - how and why? Marine Pollution Bulletin 46:385-392.

Douglas, A. E. 2008. Conflict, cheats and the persistence of symbioses. New Phytologist 177:849858.

Downs, C., K. E. McDougall, C. M. Woodley, J. E. Fauth, R. H. Richmond, A. Kushmaro, S. W. Gibb, Y. Loya, G. K. Ostrander, and E. Kramarsky-Winter. 2013. Heat-stress and light-stress induce different cellular pathologies in the symbiotic dinoflagellate during coral bleaching. PLoS ONE 8:e77173.

Drew, E. A. 1972. The biology and physiology of alga-invertebrates symbioses. II. The density of symbiotic algal cells in a number of hermatypic hard corals and alcyonarians from various depths. Journal of Experimental Marine Biology and Ecology 9:71-75.

Dromgoole, F. 1978. The effects of oxygen on dark respiration and apparent photosynthesis of marine macro-algae. Aquatic Botany 4:281-297.

Dubinsky, Z., and I. Berman-Frank. 2001. Uncoupling primary production from population growth in photosynthesizing organisms in aquatic ecosystems. Aquatic Sciences 63:4-17.

Duff, S. M., G. B. Moorhead, D. D. Lefebvre, and W. C. Plaxton. 1989. Phosphate starvation induciblebypasses' of adenylate and phosphate dependent glycolytic enzymes in Brassica nigra suspension cells. Plant Physiology 90:1275-1278.

Dunn, S. R., M. Pernice, K. Green, O. Hoegh-Guldberg, and S. G. Dove. 2012. Thermal stress promotes host mitochondrial degradation in symbiotic cnidarians: are the batteries of the reef going to run out? PLoS ONE 7:e39024.

Dunn, S. R., and V. M. Weis. 2009. Apoptosis as a post-phagocytic winnowing mechanism in a coral-dinoflagellate mutualism. Environmental Microbiology 11:268-276.

Eberhard, S., G. Finazzi, and F.-A. Wollman. 2008. The dynamics of photosynthesis. Annual Review of Genetics 42:463-515.

Edmunds, P. J., and R. D. Gates. 2008. Acclimatization in tropical reef corals. Marine Ecology Progress Series 361:307-310.

Ezzat, L., J.-F. Maguer, R. Grover, and C. Ferrier-Pagès. 2015. New insights into carbon acquisition and exchanges within the coral-dinoflagellate symbiosis under NH4+ and NO3- supply. Proceedings of the Royal Society Biology 282:20150610.

Ezzat, L., J. F. Maguer, R. Grover, and C. Ferrier-Pagès. 2016a. Limited phosphorus availability is the Achilles heel of tropical reef corals in a warming ocean. Science 6:31768.

Ezzat, L., E. Towle, J. O. Irisson, C. Langdon, and C. Ferrier-Pagès. 2016b. The relationship between heterotrophic feeding and inorganic nutrient availability in the scleractinian coral Treniformis under a short-term temperature increase. Limnology and Oceanography 61:89102.

Fabina, N. S., H. M. Putnam, E. C. Franklin, M. Stat, and R. D. Gates. 2012. Transmission mode predicts specificity and interaction patterns in coral-Symbiodinium networks. PLoS ONE 7:e44970.

Fabricius, K., K. Okaji, and G. De'Ath. 2010. Three lines of evidence to link outbreaks of the crownof-thorns seastar Acanthaster planci to the release of larval food limitation. Coral Reefs 29:593-605.

Fabricius, K. E. 2005. Effects of terrestrial runoff on the ecology of corals and coral reefs: review and synthesis. Marine Pollution Bulletin 50:125-146.

Fabricius, K. E. 2011. Factors determining the resilience of coral reefs to eutrophication: a review and conceptual model. Pages 493-505 Coral reefs: an ecosystem in transition. Springer.

Fabricius, K. E., T. F. Cooper, C. Humphrey, S. Uthicke, G. De'ath, J. Davidson, H. LeGrand, A. Thompson, and B. Schaffelke. 2012. A bioindicator system for water quality on inshore coral reefs of the Great Barrier Reef. Marine Pollution Bulletin 65:320-332.

Fabricius, K. E., G. De'ath, C. Humphrey, I. Zagorskis, and B. Schaffelke. 2013. Intra-annual variation in turbidity in response to terrestrial runoff on near-shore coral reefs of the Great Barrier Reef. Estuarine, Coastal and Shelf Science 116:57-65. 
Fabricius, K. E., O. Hoegh-Guldberg, J. E. Johnson, L. McCook, and J. Lough. 2007. Vulnerability of coral reefs of the Great Barrier Reef to climate change. Global Change Biology 24: 19781991

Falkowski, P. G. RavenJ. A.(1997) Aquatic Photosynthesis. Malden. Mass. Blackwell Science:375.

Falkowski, P. G., Z. Dubinsky, L. Muscatine, and L. McCloskey. 1993. Population control in symbiotic corals. Bioscience 43:606-611.

Falkowski, P. G., and J. A. Raven. 2007. Aquatic photosynthesis. Priceton University Press. 0691115508.

Fang, F. C. 2004. Antimicrobial reactive oxygen and nitrogen species: concepts and controversies. Nature Reviews Microbiology 2:820.

Feder, J. 1973. The phosphatases. Wiley-Liss, New York.

Ferrari, J., and F. Vavre. 2011. Bacterial symbionts in insects or the story of communities affecting communities. Philosophical Transactions of the Royal Society B: Biological Sciences 366:1389-1400.

Ferrier-Pagès, C., J. P. Gattuso, S. Dallot, and J. Jaubert. 2000. Effect of nutrient enrichment on growth and photosynthesis of the zooxanthellate coral Stylophora pistillata. Coral Reefs 19:103-113.

Ferrier-Pagès, C., V. Schoelzke, J. Jaubert, L. Muscatine, and O. Hoegh-Guldberg. 2001. Response of a scleractinian coral, Stylophora pistillata, to iron and nitrate enrichment. Journal of Experimental Marine Biology and Ecology 259:249-261.

Ferrier-Pagès, C., C. Godinot, C. D'angelo, J. Wiedenmann, and R. Grover. 2016. Phosphorus metabolism of reef organisms with algal symbionts. Ecological Monographs 86:262-277.

Finka, A., R. U. Mattoo, and P. Goloubinoff. 2011. Meta-analysis of heat-and chemically upregulated chaperone genes in plant and human cells. Cell Stress and Chaperones 16:15-31.

Fischlin, A., G. F. Midgley, L. Hughs, J. Price, R. Leemans, B. Gopal, C. Turley, M. Rounsevell, P. Dube, and J. Tarazona. 2007. Ecosystems, their properties, goods and services. Climate Change 2007: Impacts, Adaptation and Vulnerability. Contribution of Working Group II to the Fourth Assessment Report of the Intergovernmental Panel on Climate Change, M.L. Parry, O.F. Canziani, J.P. Palutikof, P.J. van der Linden and C.E. Hanson, Eds., Cambridge University Press, Cambridge, UK, 211-272

Fisher, P., M. Malme, and S. Dove. 2012. The effect of temperature stress on coral-Symbiodinium associations containing distinct symbiont types. Coral Reefs 31:473-485.

Fitt, W., and C. Cook. 2001. The effects of feeding or addition of dissolved inorganic nutrients in maintaining the symbiosis between dinoflagellates and a tropical marine cnidarian. Marine Biology 139:507-517.

Fitt, W., and M. Warner. 1995. Bleaching patterns of four species of Caribbean reef corals. The Biological Bulletin 189:298-307.

Franklin, D. J., O. Hoegh-Guldberg, R. Jones, and J. A. Berges. 2004. Cell death and degeneration in the symbiotic dinoflagellates of the coral Stylophora pistillata during bleaching. Marine Ecology Progress Series 272:117-130.

Franklin, E. C., M. Stat, X. Pochon, H. M. Putnam, and R. D. J. M. E. R. Gates. 2012. GeoSymbio: a hybrid, cloud-based web application of global geospatial bioinformatics and ecoinformatics for Symbiodinium-host symbioses. Molecular Ecology Reasources 12:369-373.

Fujise, L., H. Yamashita, G. Suzuki, K. Sasaki, L. M. Liao, and K. Koike. 2014. Moderate thermal stress causes active and immediate expulsion of photosynthetically damaged zooxanthellae (Symbiodinium) from corals. PLoS ONE 9:e114321.

Ganot, P., A. Moya, V. Magnone, D. Allemand, P. Furla, and C. Sabourault. 2011. Adaptations to endosymbiosis in a cnidarian-dinoflagellate association: differential gene expression and specific gene duplications. PLoS Genetics 7:e1002187.

Gates, R. D., O. Hoegh-Guldberg, M. J. McFall-Ngai, K. Y. Bil, and L. Muscatine. 1995. Free amino acids exhibit anthozoan" host factor" activity: They induce the release of photosynthate from 
symbiotic dinoflagellates in vitro. Proceedings of the National Academy of Sciences 92:7430-7434.

Genty, B., J.-M. Briantais, and N. R. Baker. 1989. The relationship between the quantum yield of photosynthetic electron transport and quenching of chlorophyll fluorescence. Biochimica et Biophysica Acta (BBA)-General Subjects 990:87-92.

Gierz, S. L., S. Foret, and W. Leggat. 2017. Transcriptomic Analysis of Thermally Stressed Symbiodinium Reveals Differential Expression of Stress and Metabolism Genes. Frontiers in Plant Science 8.

Gierz, S. L., B. R. Gordon, and W. Leggat. 2016. Integral light-harvesting complex expression in Symbiodinium within the coral Acropora aspera under thermal stress. Scientific Reports 6:25081.

Godinot, C., C. Ferrier-Pagès, and R. Grover. 2009. Control of phosphate uptake by zooxanthellae and host cells in the scleractinian coral Stylophora pistillata. Limnology and Oceanography 54:1627-1633.

Godinot, C., C. Ferrier-Pagès, P. Montagna, and R. Grover. 2011a. Tissue and skeletal changes in the scleractinian coral Stylophora pistillata Esper 1797 under phosphate enrichment. Journal of Experimental Marine Biology and Ecology 409:200-207.

Godinot, C., R. Grover, D. Allemand, and C. Ferrier-Pagès. 2011b. High phosphate uptake requirements of the scleractinian coral Stylophora pistillata. Journal of Experimental Biology 214:2749-2754.

Gómez, F. 2012. A checklist and classification of living dinoflagellates (Dinoflagellata, Alveolata). Cicimar Oceánides 27:65-140.

Gorbunov, M. Y., Z. S. Kolber, M. P. Lesser, and P. G. Falkowski. 2001. Photosynthesis and photoprotection in symbiotic corals. Limnology and Oceanography 46:75-85.

Goreau, T., and A. Macfarlane. 1990. Reduced growth rate of Montastrea annularis following the 1987-1988 coral-bleaching event. Coral Reefs 8:211-215.

Goulet, T. L., and M. A. Coffroth. 2003. Stability of an octocoral-algal symbiosis over time and space. Marine Ecology Progress Series 250:117-124.

Goulet, T. L., C. B. Cook, and D. Goulet. 2005. Effect of short-term exposure to elevated temperatures and light levels on photosynthesis of different host-symbiont combinations in the Aiptasia pallida/Symbiodinium symbiosis. Limnology and Oceanography 50:1490-1498.

Grant, A., M. Rémond, T. Starke-Peterkovic, and R. Hinde. 2006. A cell signal from the coral Plesiastrea versipora reduces starch synthesis in its symbiotic alga, Symbiodinium sp. Comparative Biochemistry and Physiology Part A: Molecular \& Integrative Physiology 144:458-463.

Grant, A., M. Rémond, K. Withers, and R. Hinde. 2001. Inhibition of algal photosynthesis by a symbiotic coral. Hydrobiologia 461:63-69.

Grottoli, A. G., L. J. Rodrigues, and J. E. Palardy. 2006. Heterotrophic plasticity and resilience in bleached corals. Nature 440:1186.

Grover, R., J.-F. Maguer, D. Allemand, and C. Ferrier-Pagès. 2008. Uptake of dissolved free amino acids by the scleractinian coral Stylophora pistillata. Journal of Experimental Biology 211:860-865.

Guillard, R. R., and M. S. Sieracki. 2005. Counting cells in cultures with the light microscope. Algal Culturing Techniques:239-252.

Gururani, M. A., J. Venkatesh, and L. S. P. Tran. 2015. Regulation of photosynthesis during abiotic stress-induced photoinhibition. Molecular Plant 8:1304-1320.

Hammani, K., A. Gobert, K. Hleibieh, L. Choulier, I. Small, and P. Giegé. 2011. An Arabidopsis dual-localized pentatricopeptide repeat protein interacts with nuclear proteins involved in gene expression regulation. The Plant Cell 23:730-740. 
Harland, A., and P. Davies. 1995. Symbiont photosynthesis increases both respiration and photosynthesis in the symbiotic sea anemone Anemonia viridis. Marine Biology 123:715722.

Hartl, F. U. 1996. Molecular chaperones in cellular protein folding. Nature 381:571.

Hawkins, T. D., and S. K. Davy. 2012. Nitric Oxide Production and Tolerance Differ Among Symbiodinium Types Exposed to Heat Stress. Plant and Cell Physiology 53:1889-1898.

Hawkins, T. D., and S. K. Davy. 2013. Nitric oxide and coral bleaching: is peroxynitrite generation required for symbiosis collapse? Journal of Experimental Biology 216:3185-3188.

Hawkins, T. D., T. Krueger, S. Becker, P. L. Fisher, and S. K. Davy. 2014. Differential nitric oxide synthesis and host apoptotic events correlate with bleaching susceptibility in reef corals. Coral Reefs 33:141-153.

Hawkins, T. D., T. Krueger, S. P. Wilkinson, P. L. Fisher, and S. K. Davy. 2015. Antioxidant responses to heat and light stress differ with habitat in a common reef coral. Coral Reefs 34:1229-1241.

Hikosaka, K., K. Ishikawa, A. Borjigidai, O. Muller, and Y. Onoda. 2005. Temperature acclimation of photosynthesis: mechanisms involved in the changes in temperature dependence of photosynthetic rate. Journal of Experimental Botany 57:291-302.

Hill, R., C. M. Brown, K. DeZeeuw, D. A. Campbell, and P. J. Ralph. 2011. Increased rate of D1 repair in coral symbionts during bleaching is insufficient to counter accelerated photoinactivation. Limnology and Oceanography 56:139-146.

Hill, R., C. Fernance, S. P. Wilkinson, S. K. Davy, and A. Scott. 2014. Symbiont shuffling during thermal bleaching and recovery in the sea anemone Entacmaea quadricolor. Marine Biology 161:2931-2937.

Hill, R., C. Frankart, and P. J. Ralph. 2005. Impact of bleaching conditions on the components of non-photochemical quenching in the zooxanthellae of a coral. Journal of Experimental Marine Biology and Ecology 322:83-92.

Hill, R., A. W. Larkum, C. Frankart, M. Kühl, and P. J. Ralph. 2004. Loss of functional Photosystem II reaction centres in zooxanthellae of corals exposed to bleaching conditions: using fluorescence rise kinetics. Photosynthesis Research 82:59-72.

Hill, R., and P. J. Ralph. 2008. Dark-induced reduction of the plastoquinone pool in zooxanthellae of scleractinian corals and implications for measurements of chlorophyll a fluorescence. SYMBIOSIS-REHOVOT- 46:45.

Hillyer, K. E., D. A. Dias, A. Lutz, S. P. Wilkinson, U. Roessner, and S. K. Davy. 2017. Metabolite profiling of symbiont and host during thermal stress and bleaching in the coral Acropora aspera. Coral Reefs 36:105-118.

Hillyer, K. E., S. Tumanov, S. Villas-Boas, and S. K. Davy. 2016a. Metabolite profiling of symbiont and host during thermal stress and bleaching in a model cnidarian-dinoflagellate symbiosis. Journal of Experimental Biology 219:516-527.

Hillyer, K. E., S. Tumanov, S. Villas-Bôas, and S. K. Davy. 2016b. Metabolite profiling of symbiont and host during thermal stress and bleaching in a model cnidarian-dinoflagellate symbiosis. Journal of Experimental Biology 219:516-527.

Hirose, M., H. Yamamoto, and M. Nonaka. 2008. Metamorphosis and acquisition of symbiotic algae in planula larvae and primary polyps of Acropora spp. Coral Reefs 27:247-254.

Hochachka PW, S. 2002. Biochemical adaptations. Oxford University Press, Oxford.

Hoefnagel, M. H., O. K. Atkin, and J. T. Wiskich. 1998. Interdependence between chloroplasts and mitochondria in the light and the dark. Biochimica et Biophysica Acta (BBA)-Bioenergetics 1366:235-255.

Hoegh-Guldberg, O. 1999. Climate change, coral bleaching and the future of the world's coral reefs. Marine and Freshwater Research 50:839-866.

Hoegh-Guldberg, O., R. J. Jones, S. Ward, and W. K. Loh. 2002. Ecology (Communication arising): Is coral bleaching really adaptive? Nature 415:601. 
Hoegh-Guldberg, O., L. McCloskey, and L. Muscatine. 1987. Expulsion of zooxanthellae by symbiotic cnidarians from the Red Sea. Coral Reefs 5:201-204.

Hoegh-Guldberg, O., P. J. Mumby, A. J. Hooten, R. S. Steneck, P. Greenfield, E. Gomez, C. D. Harvell, P. F. Sale, A. J. Edwards, and K. Caldeira. 2007. Coral reefs under rapid climate change and ocean acidification. Science 318:1737-1742.

Hoegh-Guldberg, O., L. Muscatine, C. Goiran, D. Siggaard, and G. Marion. 2004. Nutrient-induced perturbations to delta $\mathrm{C}-13$ and delta $\mathrm{N}-15$ in symbiotic dinoilagellates and their coral hosts. Marine Ecology Progress Series 280:105-114.

Hoegh-Guldberg, O., and G. J. Smith. 1989a. The effect of sudden changes in temperature, light and salinity on the population density and export of zooxanthellae from the reef corals Stylophora pistillata Esper and Seriatopora hystrix Dana. Journal of Experimental Marine Biology and Ecology 129:279-303.

Hoegh-Guldberg, O., and G. J. Smith. 1989b. Influence of the population density of zooxanthellae and supply of ammonium on the biomass and metabolic characteristics of the reef corals Seriatopora hystrix and Stylophora pistillata. Marine Ecology Progress Series:173-186.

Holcomb, M., D. C. McCorkle, and A. L. Cohen. 2010. Long-term effects of nutrient and CO2 enrichment on the temperate coral Astrangia poculata (Ellis and Solander, 1786). Journal of Experimental Marine Biology and Ecology 386:27-33.

Hou, Y., and S. Lin. 2009. Distinct gene number-genome size relationships for eukaryotes and noneukaryotes: gene content estimation for dinoflagellate genomes. PLoS ONE 4:e6978.

Houten, S. M., and R. J. Wanders. 2010. A general introduction to the biochemistry of mitochondrial fatty acid $\beta$-oxidation. Journal of Inherited Metabolic Disease 33:469-477.

Howarth, R., F. Chan, D. J. Conley, J. Garnier, S. C. Doney, R. Marino, and G. Billen. 2011. Coupled biogeochemical cycles: eutrophication and hypoxia in temperate estuaries and coastal marine ecosystems. Frontiers in Ecology and the Environment 9:18-26.

Howarth, R. W., G. Billen, D. Swaney, A. Townsend, N. Jaworski, K. Lajtha, J. A. Downing, R. Elmgren, N. Caraco, and T. Jordan. 1996. Regional nitrogen budgets and riverine N \& P fluxes for the drainages to the North Atlantic Ocean: Natural and human influences. Pages 75-139 Nitrogen cycling in the North Atlantic Ocean and its watersheds. Springer.

Howells, E., V. Beltran, N. Larsen, L. Bay, B. Willis, and M. J. N. C. C. Van Oppen. 2012. Coral thermal tolerance shaped by local adaptation of photosymbionts. Nature 2:116.

Howells, E., M. Van Oppen, and B. Willis. 2009. High genetic differentiation and cross-shelf patterns of genetic diversity among Great Barrier Reef populations of Symbiodinium. Coral Reefs 28:215-225.

Huang, A. H. 2018. Plant lipid droplets and their associated proteins: potential for rapid advances. Plant Physiology 176:1894-1918.

Hughes, T. P., A. H. Baird, D. R. Bellwood, M. Card, S. R. Connolly, C. Folke, R. Grosberg, O. Hoegh-Guldberg, J. B. Jackson, and J. Kleypas. 2003. Climate change, human impacts, and the resilience of coral reefs. Science 301:929-933.

Hughes, T. P., J. T. Kerry, M. Álvarez-Noriega, J. G. Álvarez-Romero, K. D. Anderson, A. H. Baird, R. C. Babcock, M. Beger, D. R. Bellwood, and R. Berkelmans. 2017. Global warming and recurrent mass bleaching of corals. Nature 543:373.

Hume, B., D’angelo, C., Burt, J., Baker, A. C., Riegl, B., \& Wiedenmann, J. 2013. Corals from the Persian/Arabian Gulf as models for thermotolerant reef-builders: prevalence of clade C3 Symbiodinium, host fluorescence and ex situ temperature tolerance. Marine Pollution Bulletin, 72: 313-322.

Hume, B. C., C. D'Angelo, E. G. Smith, J. R. Stevens, J. Burt, and J. J. S. r. Wiedenmann. 2015. Symbiodinium thermophilum sp. nov., a thermotolerant symbiotic alga prevalent in corals of the world's hottest sea, the Persian/Arabian Gulf. Nature 5:8562.

Hwang, I., and D. G. Robinson. 2009. Transport vesicle formation in plant cells. Current Opinion in Plant Biology 12:660-669. 
Iglesias-Prieto, R., J. L. Matta, W. A. Robins, and R. K. Trench. 1992. Photosynthetic response to elevated temperature in the symbiotic dinoflagellate Symbiodinium microadriaticum in culture. Proceedings of the National Academy of Sciences 89:10302-10305.

Jackson, A. E., and D. Yellowlees. 1990. Phosphate uptake by zooxanthellae isolated from corals. Proceedings of the Royal Society of Biology London 242:201-204.

Jeffries, P., S. Gianinazzi, S. Perotto, K. Turnau, and J.-M. Barea. 2003. The contribution of arbuscular mycorrhizal fungi in sustainable maintenance of plant health and soil fertility. Biology and Fertility of Soils 37:1-16.

Jiang, P.-L., B. Pasaribu, and C.-S. Chen. 2014. Nitrogen-deprivation elevates lipid levels in Symbiodinium spp. by lipid droplet accumulation: morphological and compositional analyses. PLoS ONE 9:e87416.

Johnson, J. E., P. A. Marshall, and G. B. R. M. P. Authority. 2007. Climate change and the Great Barrier Reef: a vulnerability assessment. Great Barrier Reef Marine Park Authority and the Australian Greenhouse Office.

Jokiel, P. L. 2004. Temperature stress and coral bleaching. Pages 401-425 Coral health and disease. Springer.

Jones, B., and M. Nishiguchi. 2004. Counterillumination in the hawaiian bobtail squid, Euprymna scolopes Berry (Mollusca: Cephalopoda). Marine Biology 144:1151-1155.

Jones, R., and O. Hoegh-Guldberg. 2001. Diurnal changes in the photochemical efficiency of the symbiotic dinoflagellates (Dinophyceae) of corals: photoprotection, photoinactivation and the relationship to coral bleaching. Plant, Cell \& Environment 24:89-99.

Jones, R. J., O. Hoegh-Guldberg, A. W. Larkum, and U. Schreiber. 1998. Temperature-induced bleaching of corals begins with impairment of the $\mathrm{CO} 2$ fixation mechanism in zooxanthellae. Plant, Cell \& Environment 21:1219-1230.

Jones, R. J., S. Ward, A. Y. Amri, and O. Hoegh-Guldberg. 2000. Changes in quantum efficiency of Photosystem II of symbiotic dinoflagellates of corals after heat stress, and of bleached corals sampled after the 1998 Great Barrier Reef mass bleaching event. Marine and Freshwater Research 51:63-71.

Jørgensen, R., A. R. Merrill, and G. R. Andersen. 2006. The life and death of translation elongation factor 2. Portland Press Limited.

Kaida, R., Y. Satoh, V. Bulone, Y. Yamada, T. Kaku, T. Hayashi, and T. S. Kaneko. 2009. Activation of $\beta$-glucan synthases by wall-bound purple acid phosphatase in tobacco cells. Plant Physiology 150:1822-1830.

Kanazawa, A., G. J. Blanchard, M. Szabo, P. J. Ralph, and D. M. Kramer. 2014. The site of regulation of light capture in Symbiodinium: Does the peridinin-chlorophyll alpha-protein detach to regulate light capture? Biochimica Et Biophysica Acta-Bioenergetics 1837:12271234.

Kaniewska, P., C.-K. K. Chan, D. Kline, E. Y. S. Ling, N. Rosic, D. Edwards, O. Hoegh-Guldberg, and S. Dove. 2015. Transcriptomic changes in coral holobionts provide insights into physiological challenges of future climate and ocean change. PLoS ONE 10:e0139223.

Kaplan, F., J. Kopka, D. W. Haskell, W. Zhao, K. C. Schiller, N. Gatzke, D. Y. Sung, and C. L. Guy. 2004. Exploring the temperature-stress metabolome of Arabidopsis. Plant Physiology 136:4159-4168.

Karako-Lampert, S., D. Katcoff, Y. Achituv, Z. Dubinsky, and N. Stambler. 2005. Responses of Symbiodinium microadriaticum clade B to different environmental conditions. Journal of Experimental Marine Biology and Ecology 318:11-20.

Karim, W., S. Nakaema, and M. Hidaka. 2015. Temperature effects on the growth rates and photosynthetic activities of Symbiodinium cells. Journal of Marine Science and Engineering 3:368-381. 
Kazandjian, A., V. A. Shepherd, M. Rodriguez-Lanetty, W. Nordemeier, A. W. Larkum, and R. G. Quinnell. 2008. Isolation of symbiosomes and the symbiosome membrane complex from the zoanthid Zoanthus robustus. Phycologia 47:294-306.

Kinsey, D. W., and P. J. Davies. 1979. Effects of elevated nitrogen and phosphorus on coral reef growth. Limnology and Oceanography 24:935-940.

Kitajima, M., and W. Butler. 1975. Quenching of chlorophyll fluorescence and primary photochemistry in chloroplasts by dibromothymoquinone. Biochimica et Biophysica Acta (BBA)-Bioenergetics 376:105-115.

Koop, K., D. Booth, A. Broadbent, J. Brodie, D. Bucher, D. Capone, J. Coll, W. Dennison, M. Erdmann, P. Harrison, O. Hoegh-Guldberg, P. Hutchings, G. B. Jones, A. W. D. Larkum, J. O'Neil, A. Steven, E. Tentori, S. Ward, J. Williamson, and D. Yellowlees. 2001. ENCORE: The effect of nutrient enrichment on coral reefs. Synthesis of results and conclusions. Marine Pollution Bulletin 42:91-120.

Kopp, C., M. Pernice, I. Domart-Coulon, C. Djediat, J. E. Spangenberg, D. T. L. Alexander, M. Hignette, T. Meziane, and A. Meibom. 2013. Highly Dynamic Cellular-Level Response of Symbiotic Coral to a Sudden Increase in Environmental Nitrogen. Marine Biology 4.

Kopp, C., M. Wisztorski, J. Revel, M. Mehiri, V. Dani, L. Capron, D. Carette, I. Fournier, L. Massi, D. Mouajjah, S. Pagnotta, F. Priouzeau, M. Salzet, A. Meibom, and C. Sabourault. 2015. MALDI-MS and NanoSIMS imaging techniques to study cnidarian-dinoflagellate symbioses. Zoology 118:125-131.

Krämer, W. E., I. Caamaño-Ricken, C. Richter, and K. Bischof. 2012. Dynamic regulation of photoprotection determines thermal tolerance of two phylotypes of Symbiodinium clade A at two photon fluence rates. Photochemistry and Photobiology 88:398-413.

Krause, G., and E. Weis. 1991. Chlorophyll fluorescence and photosynthesis: the basics. Annual Review of Plant Biology 42:313-349.

Krediet, C. J., J. C. DeNofrio, C. Caruso, M. S. Burriesci, K. Cella, and J. R. Pringle. 2015. Rapid, precise, and accurate counts of symbiodinium cells using the guava flow cytometer, and a comparison to other methods. PLoS ONE 10:e0135725.

Kromer, S. 1995. Respiration during photosynthesis. Annual Review of Plant Biology 46:45-70.

Kromer, S., G. Malmberg, and P. Gardestrom. 1993. Mitochondrial Contribution to Photosynthetic Metabolism (A Study with Barley (Hordeum vulgare L.) Leaf Protoplasts at Different Light Intensities and CO2 Concentrations). Plant Physiology 102:947-955.

Kroon, F. J., P. M. Kuhnert, B. L. Henderson, S. N. Wilkinson, A. Kinsey-Henderson, B. Abbott, J. E. Brodie, and R. D. Turner. 2012. River loads of suspended solids, nitrogen, phosphorus and herbicides delivered to the Great Barrier Reef lagoon. Marine Pollution Bulletin 65:167-181.

Krueger, T., S. Becker, S. Pontasch, S. Dove, O. Hoegh-Guldberg, W. Leggat, P. L. Fisher, and S. K. Davy. 2014. Antioxidant plasticity and thermal sensitivity in four types of Symbiodinium sp. Journal of Phycology 50:1035-1047.

Krueger, T., T. D. Hawkins, S. Becker, S. Pontasch, S. Dove, O. Hoegh-Guldberg, W. Leggat, P. L. Fisher, and S. K. Davy. 2015. Differential coral bleaching - Contrasting the activity and response of enzymatic antioxidants in symbiotic partners under thermal stress. Comparative Biochemistry and Physiology Part A: Molecular \& Integrative Physiology 190:15-25.

LaJeunesse, T. C. 2001. Investigating the biodiversity, ecology, and phylogeny of endosymbiotic dinoflagellates in the genus Symbiodinium using the ITS region: in search of a "species" level marker. Journal of Phycology 37:866-880.

LaJeunesse, T. C., G. Lambert, R. A. Andersen, M. A. Coffroth, and D. W. Galbraith. 2005. Symbiodinium (pyrrhophyta) genome sizes (dna content) are smallest among dinoflagellates 1. Journal of Phycology 41:880-886.

LaJeunesse, T. C., J. E. Parkinson, P. W. Gabrielson, H. J. Jeong, J. D. Reimer, C. R. Voolstra, and S. R. J. C. B. Santos. 2018. Systematic Revision of Symbiodiniaceae Highlights the Antiquity and Diversity of Coral Endosymbionts. 
LaJeunesse, T. C., R. Smith, M. Walther, J. Pinzón, D. T. Pettay, M. McGinley, M. Aschaffenburg, P. Medina-Rosas, A. L. Cupul-Magaña, and A. L. Pérez. 2010. Host-symbiont recombination versus natural selection in the response of coral-dinoflagellate symbioses to environmental disturbance. Proceedings of the Royal Society of London: Biological Sciences 277:29252934.

LaJeunesse, T. C., R. T. Smith, J. Finney, and H. J. Oxenford. 2009. Outbreak and persistence of opportunistic symbiotic dinoflagellates during the 2005 Caribbean mass coral 'bleaching'event. Proceedings of the Royal Society of Biological Sciences 267.1676: 41394148.

Lange, H., J. Bavouzet, P. Taillandier, and C. Delorme. 1993. Systematic error and comparison of four methods for assessing the viability ofSaccharomyces cerevisiae suspensions. Biotechnology Techniques 7:223-228.

Lapointe, B. E. 1987. Phosphorus-and nitrogen-limited photosynthesis and growth of Gracilaria tikvahiae (Rhodophyceae) in the Florida Keys: an experimental field study. Marine Biology 93:561-568.

Lapointe, B. E. 1997. Nutrient thresholds for bottom-up control of macroalgal blooms on coral reefs in Jamaica and southeast Florida. Limnology and Oceanography 42:1119-1131.

Lapointe, B. E., and J. O'Connell. 1989. Nutrient-enhanced growth of Cladophora prolifera in Harrington Sound, Bermuda: eutrophication of a confined, phosphorus-limited marine ecosystem. Estuarine, Coastal and Shelf Science 28:347-360.

Leggat, W., M. R. Badger, and D. Yellowlees. 1999. Evidence for an inorganic carbon-concentrating mechanism in the symbiotic dinoflagellate Symbiodinium sp. Plant Physiology 121:12471255.

Leggat, W., E. M. Marendy, B. Baillie, S. M. Whitney, M. Ludwig, M. R. Badger, and D. Yellowlees. 2002. Dinoflagellate symbioses: strategies and adaptations for the acquisition and fixation of inorganic carbon. Functional Plant Biology 29:309-322.

Leggat, W., D. Yellowlees, and M. Medina. 2011. Recent progress in Symbiodinium transcriptomics. Journal of Experimental Marine Biology and Ecology 408:120-125.

Lehninger, A. L., D. L. Nelson, and M. M. Cox. 1993. Principles of biochemistry W.H. Freeman and Company, New York, USA.

Lesser, M. P. 1996. Elevated temperatures and ultraviolet radiation cause oxidative stress and inhibit photosynthesis in ymbiotic dinoflagellates. Limnology and Oceanography 41:271-283.

Lesser, M. P. 1997. Oxidative stress causes coral bleaching during exposure to elevated temperatures. Coral Reefs 16:187-192.

Lesser, M. P. 2006. Oxidative stress in marine environments: biochemistry and physiological ecology. Annual Revisions of Physiology. 68:253-278.

Lesser, M. P., and J. H. Farrell. 2004. Exposure to solar radiation increases damage to both host tissues and algal symbionts of corals during thermal stress. Coral Reefs 23:367-377.

Levine, B., and D. J. Klionsky. 2004. Development by self-digestion: molecular mechanisms and biological functions of autophagy. Developmental Cell 6:463-477.

Lewis, C. L., and M. A. Coffroth. 2004. The acquisition of exogenous algal symbionts by an octocoral after bleaching. Science 304:1490-1492.

Li, K., C. Xu, Z. Li, K. Zhang, A. Yang, and J. Zhang. 2008. Comparative proteome analyses of phosphorus responses in maize (Zea mays L.) roots of wild-type and a low-P-tolerant mutant reveal root characteristics associated with phosphorus efficiency. The Plant Journal 55:927939.

Li, K., C. Xu, K. Zhang, A. Yang, and J. Zhang. 2007. Proteomic analysis of roots growth and metabolic changes under phosphorus deficit in maize (Zea mays L.) plants. Proteomics 7:1501-1512.

Li, M., X. Shi, C. Guo, and S. Lin. 2016. Phosphorus deficiency inhibits cell division but not growth in the dinoflagellate Amphidinium carterae. Frontiers in Microbiology 7:826. 
Liang, C., J. Tian, and H. Liao. 2013. Proteomics dissection of plant responses to mineral nutrient deficiency. Proteomics 13:624-636.

Lichtenthaler, H. K. 1988. Applications of chlorophyll fluorescence in photosynthesis research, stress physiology, hydrobiology and remote sensing. Kluwer Academic Publishers.

Lichtenthaler, H. K., and C. Buschmann. 2001. Chlorophylls and carotenoids: Measurement and characterization by UV-VIS spectroscopy. Current Protocols in Analytical Chemistry.

Lilley, R., P. J. Ralph, and A. W. Larkum. 2010. The determination of activity of the enzyme Rubisco in cell extracts of the dinoflagellate alga Symbiodinium sp. by manganese chemiluminescence and its response to short-term thermal stress of the alga. Plant, Cell \& Environment 33:995-1004.

Lim, H., M.-H. Cho, J.-S. Jeon, S. H. Bhoo, Y.-K. Kwon, and T.-R. Hahn. 2009. Altered expression of pyrophosphate: fructose-6-phosphate 1-phosphotransferase affects the growth of transgenic Arabidopsis plants. Molecules and Cells 27:641-649.

Littman, R. A., M. J. van Oppen, and B. L. Willis. 2008. Methods for sampling free-living Symbiodinium (zooxanthellae) and their distribution and abundance at Lizard Island (Great Barrier Reef). Journal of Experimental Marine Biology and Ecology 364:48-53.

Loya, Y., H. Lubinevsky, M. Rosenfeld, and E. Kramarsky-Winter. 2004. Nutrient enrichment caused by in situ fish farms at Eilat, Red Sea is detrimental to coral reproduction. Marine Pollution Bulletin 49:344-353.

Lund, J., C. Kipling, and E. Le Cren. 1958. The inverted microscope method of estimating algal numbers and the statistical basis of estimations by counting. Hydrobiologia 11:143-170.

Lutz, A., J.-B. Raina, C. A. Motti, D. J. Miller, and M. J. van Oppen. 2015. Host coenzyme Q redox state is an early biomarker of thermal stress in the coral Acropora millepora. PLoS ONE 10:e0139290.

Madlung, A., and L. Comai. 2004. The effect of stress on genome regulation and structure. Annalysis of Botany 94:481-495.

Malinova, I., H.-H. Kunz, S. Alseekh, K. Herbst, A. R. Fernie, M. Gierth, and J. Fettke. 2014. Reduction of the cytosolic phosphoglucomutase in Arabidopsis reveals impact on plant growth, seed and root development, and carbohydrate partitioning. PLoS ONE 9:e112468.

Malumbres, M., and M. Barbacid. 2005. Mammalian cyclin-dependent kinases. Trends in Biochemical Sciences 30:630-641.

Man, S. M., and T.-D. Kanneganti. 2016. Converging roles of caspases in inflammasome activation, cell death and innate immunity. Nature Reviews Immunology 16:7.

Marschner, H., and B. Dell. 1994. Nutrient uptake in mycorrhizal symbiosis. Plant and soil 159:89102.

Marubini, F., and P. Davies. 1996. Nitrate increases zooxanthellae population density and reduces skeletogenesis in corals. Marine Biology 127:319-328.

Marx, M.-C., M. Wood, and S. Jarvis. 2001. A microplate fluorimetric assay for the study of enzyme diversity in soils. Soil Biology and Biochemistry 33:1633-1640.

Mathur, S., D. Agrawal, and A. Jajoo. 2014. Photosynthesis: response to high temperature stress. Journal of Photochemistry and Photobiology: Biology 137:116-126.

Matthews, J. L., C. M. Crowder, C. A. Oakley, A. Lutz, U. Roessner, E. Meyer, A. R. Grossman, V. M. Weis, and S. K. Davy. 2017. Optimal nutrient exchange and immune responses operate in partner specificity in the cnidarian-dinoflagellate symbiosis. Proceedings of the National Academy of Sciences of the United States of America 114:13194-13199.

Matthews, J. L., C. A. Oakley, A. Lutz, K. E. Hillyer, U. Roessner, A. R. Grossman, V. M. Weis, and S. K. Davy. 2018. Partner switching and metabolic flux in a model cnidarian-dinoflagellate symbiosis. Proceedings of the Royal Society of Biology 285:20182336.

Maxwell, K., and G. N. Johnson. 2000. Chlorophyll fluorescence-a practical guide. Journal of Experimental Botany 51:659-668. 
McAuley, P., and D. C. Smith. 1982. The green hydra symbiosis. V. Stages in the intracellular recognition of algal symbionts by digestive cells. Proceedings of the Royal Society of London Biology 216:7-23.

McClanahan, T. 2004. The relationship between bleaching and mortality of common corals. Marine Biology 144:1239-1245.

McCloskey, L., T. G. Cove, and E. A. Verde. 1996. Symbiont expulsion from the anemone Anthopleura elegantissima (Brandt)(Cnidaria; Anthozoa). Journal of Experimental Marine Biology and Ecology 195:173-186.

McGinley, M. P., M. D. Aschaffenburg, D. T. Pettay, R. T. Smith, T. C. LaJeunesse, and M. E. Warner. 2012a. Symbiodinium spp. in colonies of eastern Pacific Pocillopora spp. are highly stable despite the prevalence of low-abundance background populations. Marine Ecology Progress Series 462:1-7.

McGinley, M. P., M. D. Aschaffenburg, D. T. Pettay, R. T. Smith, T. C. LaJeunesse, and M. E. Warner. 2012b. Transcriptional response of two core photosystem genes in Symbiodinium spp. exposed to thermal stress. PLoS ONE 7:e50439.

McGinty, E. S., J. Pieczonka, and L. D. J. M. e. Mydlarz. 2012. Variations in reactive oxygen release and antioxidant activity in multiple Symbiodinium types in response to elevated temperature. Microbial Ecology 64:1000-1007.

McGuire, M., and A. Szmant. 1997. Time course of physiological responses to NH4 enrichment by a coral-zooxanthellae symbiosis. Pages 909-914 in Proceedings of the 8th International Coral Reef Symposium.

Menkveld, R., J. VANZYL, and T. v. W. Kotze. 1984. A statistical comparison of three methods for the counting of human spermatozoa. Andrologia 16:554-558.

Merle, P.-L., C. Sabourault, S. Richier, D. Allemand, and P. Furla. 2007. Catalase characterization and implication in bleaching of a symbiotic sea anemone. Free Radical Biology and Medicine 42:236-246.

Meyer, E., and V. M. Weis. 2012. Study of Cnidarian-Algal Symbiosis in the "Omics" Age. Biological Bulletin 223:44-65.

Middlebrook, R., K. R. Anthony, O. Hoegh-Guldberg, and S. Dove. 2010. Heating rate and symbiont productivity are key factors determining thermal stress in the reef-building coral Acropora formosa. Journal of Experimental Biology 213:1026-1034.

Middlebrook, R., O. Hoegh-Guldberg, and W. Leggat. 2008. The effect of thermal history on the susceptibility of reef-building corals to thermal stress. Journal of Experimental Biology 211:1050-1056.

Miller, D., and D. Yellowlees. 1989. Inorganic nitrogen uptake by symbiotic marine cnidarians: a critical review. Proceedings of the Royal Society of London B. 237:109-125.

Møller, M., S. Myklestad, and A. Haug. 1975. Alkaline and acid phosphatases of the marine diatoms Chaetoceros affinis var. willei (Gran) Hustedt and Skeletonema costatum (Grev.) Cleve. Journal of Experimental Marine Biology and Ecology 19:217-226.

Moore, F., and B. Best. 2001. Coral reef crisis: causes and consequences. Pages 5-10 in Global Trade and Consumer Choices: Coral Reefs in Crisis, Proceedings of an American Association for the Advancement of Science (AAAS) Meeting.

Moustafa, A., A. N. Evans, D. M. Kulis, J. D. Hackett, D. L. Erdner, D. M. Anderson, and D. Bhattacharya. 2010. Transcriptome profiling of a toxic dinoflagellate reveals a gene-rich protist and a potential impact on gene expression due to bacterial presence. PLoS One 5:e9688.

Müller, R., M. Morant, H. Jarmer, L. Nilsson, and T. H. Nielsen. 2007. Genome-wide analysis of the Arabidopsis leaf transcriptome reveals interaction of phosphate and sugar metabolism. Plant Physiology 143:156-171. 
Munson, M. A., P. Baumann, and M. G. Kinsey. 1991. Buchnera gen. nov. and Buchnera aphidicola sp. nov., a taxon consisting of the mycetocyte-associated, primary endosymbionts of aphids. International Journal of Systematic and Evolutionary Microbiology 41:566-568.

Muscatine, L., P. Falkowski, Z. Dubinsky, P. Cook, and L. McCloskey. 1989a. The effect of external nutrient resources on the population dynamics of zooxanthellae in a reef coral. Proceedigns of the Royal Society of London. B 236:311-324.

Muscatine, L., P. Falkowski, J. Porter, and Z. Dubinsky. 1984. Fate of photosynthetic fixed carbon in light-and shade-adapted colonies of the symbiotic coral Stylophora pistillata. Proceedigns of the Royal Society of London. B 222:181-202.

Muscatine, L., C. Ferrier-Pagès, A. Blackburn, R. Gates, G. Baghdasarian, and D. Allemand. 1998. Cell-specific density of symbiotic dinoflagellates in tropical anthozoans. Coral Reefs 17:329337.

Muscatine, L., C. Goiran, L. Land, J. Jaubert, J.-P. Cuif, and D. Allemand. 2005. Stable isotopes $(\delta 13 \mathrm{C}$ and $\delta 15 \mathrm{~N})$ of organic matrix from coral skeleton. Proceedings of the National Academy of Sciences of the United States of America 102:1525-1530.

Muscatine, L., R. Pool, and E. Cernichiari. 1972. Some factors influencing selective release of soluble organic material by zooxanthellae from reef corals. Marine Biology 13:298-308.

Muscatine, L., J. Porter, and I. Kaplan. 1989b. Resource partitioning by reef corals as determined from stable isotope composition. Marine Biology 100:185-193.

Muscatine, L., and J. W. Porter. 1977. Reef corals - mutualistic symbioses adapted to nutrient-poor environments. Bioscience 27:454-460.

Muscatine, L., L. R McCloskey, and R. E Marian. 1981. Estimating the daily contribution of carbon from zooxanthellae to coral animal respiration. Limnology and Oceanography 26:601-611.

Mydlarz, L. D., L. Fuess, W. Mann, J. H. Pinzón, and D. J. Gochfeld. 2016. Cnidarian immunity: from genomes to phenomes. Pages 441-466 The Cnidaria, Past, Present and Future. Springer.

Niedzwiedzki, D. M., J. Jiang, C. S. Lo, and R. E. Blankenship. 2014. Spectroscopic properties of the Chlorophyll a-Chlorophyll c2-Peridinin-Protein-Complex (acpPC) from the coral symbiotic dinoflagellate Symbiodinium. Photosynthesis Research 120:125-139.

Nishiyama, Y., and N. Murata. 2014. Revised scheme for the mechanism of photoinhibition and its application to enhance the abiotic stress tolerance of the photosynthetic machinery. Applied Microbiology and Biotechnology 98:8777-8796.

Noguchi, K., and K. Yoshida. 2008. Interaction between photosynthesis and respiration in illuminated leaves. Mitochondrion 8:87-99.

Nordemar, I., M. Nyström, and R. Dizon. 2003. Effects of elevated seawater temperature and nitrate enrichment on the branching coral Porites cylindrica in the absence of particulate food. Marine Biology 142:669-677.

O’Neil, J. M., and D. G. Capone. 2008. Nitrogen cycling in coral reef environments. Nitrogen in the Marine Environment, Eds.: Capone, DG, Bronk, DA, Mulholland, MR, and Carpenter, EJ:949-989.

Oakley, C., and S. Davy. 2018. Cell Biology of Coral Bleaching. Pages 189-211 Coral Bleaching. Springer.

Oakley, C. A., M. F. Ameismeier, L. Peng, V. M. Weis, A. R. Grossman, and S. K. Davy. 2016. Symbiosis induces widespread changes in the proteome of the model cnidarian Aiptasia. Cellular Microbiology 18:1009-1023.

Oakley, C. A., E. Durand, S. P. Wilkinson, L. F. Peng, V. M. Weis, A. R. Grossman, and S. K. Davy. 2017. Thermal Shock Induces Host Proteostasis Disruption and Endoplasmic Reticulum Stress in the Model Symbiotic Cnidarian Aiptasia. Journal of Proteome Research 16:21212134.

Oakley, C. A., B. M. Hopkinson, and G. W. Schmidt. 2014a. Mitochondrial terminal alternative oxidase and its enhancement by thermal stress in the coral symbiont Symbiodinium. Coral Reefs 33:543-552. 
Oakley, C. A., G. W. Schmidt, and B. M. Hopkinson. 2014b. Thermal responses of Symbiodinium photosynthetic carbon assimilation. Coral Reefs 33:501-512.

Odum, H. T., and E. P. Odum. 1955. Trophic structure and productivity of a windward coral reef community on Eniwetok Atoll. Ecological Monographs 25:291-320.

Ohad, I., N. Keren, H. Zer, H. Gong, T. S. Mor, A. Gal, S. Tal, and Y. Domovich. 1994. Lightinduced degradation of the photosystem II reaction centre D1 protein in vivo: an integrative approach. Photoinhibition from the Molecule to the Field, N. Baker, ed (Oxford, UK: Bios Scientific Publishers):161-177.

Okamoto, O. K., D. L. Robertson, T. F. Fagan, J. W. Hastings, and P. Colepicolo. 2001. Different regulatory mechanisms modulate the expression of a dinoflagellate iron-superoxide dismutase. Journal of Biological Chemistry 276:19989-19993.

Padilla-Gamiño, J. L., X. Pochon, C. Bird, G. T. Concepcion, and R. D. Gates. 2012. From parent to gamete: vertical transmission of Symbiodinium (Dinophyceae) ITS2 sequence assemblages in the reef building coral Montipora capitata. PLoS ONE 7:e38440.

Palstra, F. P., and D. E. Ruzzante. 2008. Genetic estimates of contemporary effective population size: what can they tell us about the importance of genetic stochasticity for wild population persistence? Molecular Ecology 17:3428-3447.

Palumbi, S. R. 2003. Population genetics, demographic connectivity, and the design of marine reserves. Ecological Applications 13:146-158.

Pandolfi, J. M. 2015. Incorporating uncertainty in predicting the future response of coral reefs to climate change. Annual Review of Ecology, Evolution, and Systematics 46:281-303.

Parkhill, J. P., G. Maillet, and J. J. Cullen. 2001. Fluorescence-based maximal quantum yield for PSII as a diagnostic of nutrient stress. Journal of Phycology 37:517-529.

Parkinson, J. E., A. T. Banaszak, N. S. Altman, T. C. LaJeunesse, and I. B. Baums. 2015. Intraspecific diversity among partners drives functional variation in coral symbioses. Scientific Reports 5.

Parkinson, J. E., S. Baumgarten, C. T. Michell, I. B. Baums, T. C. LaJeunesse, and C. R. Voolstra. 2016. Gene expression variation resolves species and individual strains among coralassociated dinoflagellates within the genus Symbiodinium. Genome Biology and Evolution 8:665-680.

Parkinson, J. E., and I. B. Baums. 2014. The extended phenotypes of marine symbioses: ecological and evolutionary consequences of intraspecific genetic diversity in coral-algal associations. Frontiers in Microbiology 5:445.

Pasaribu, B., I.-P. Lin, J. T. Tzen, G.-Y. Jauh, T.-Y. Fan, Y.-M. Ju, J.-O. Cheng, C.-S. Chen, and P.L. Jiang. 2014. SLDP: a novel protein related to caleosin is associated with the endosymbiotic Symbiodinium lipid droplets from Euphyllia glabrescens. Marine biotechnology 16:560-571.

Pasaribu, B., L.-C. Weng, I.-P. Lin, E. Camargo, J. T. Tzen, C.-H. Tsai, S.-L. Ho, M.-R. Lin, L.-H. Wang, and C.-S. Chen. 2015. Morphological variability and distinct protein profiles of cultured and endosymbiotic Symbiodinium cells isolated from Exaiptasia pulchella. Scientific Reports 5:15353.

Paxton, C. W., S. K. Davy, and V. M. Weis. 2013. Stress and death of cnidarian host cells play a role in cnidarian bleaching. Journal of Experimental Biology 216:2813-2820.

Pérez, M., and J. Romero. 1993. Preliminary data on alkaline phosphatase activity associated with Mediterranean seagrasses. Botanica Marina 36:499-502.

Perez, S., and V. Weis. 2006. Nitric oxide and cnidarian bleaching: an eviction notice mediates breakdown of a symbiosis. Journal of Experimental Biology 209:2804-2810.

Pernice, M., A. Meibom, A. Van Den Heuvel, C. Kopp, I. Domart-Coulon, O. Hoegh-Guldberg, and S. Dove. 2012. A single-cell view of ammonium assimilation in coral-dinoflagellate symbiosis. The ISME journal 6:1314. 
Peterson, M. E., R. M. Daniel, M. J. Danson, and R. Eisenthal. 2007. The dependence of enzyme activity on temperature: determination and validation of parameters. Biochemical Journal 402:331-337.

Pimpl, P., A. Movafeghi, S. Coughlan, J. Denecke, S. Hillmer, and D. G. Robinson. 2000. In situ localization and in vitro induction of plant COPI-coated vesicles. The Plant Cell 12:22192235.

Plaxton, W. C. 1996. The organization and regulation of plant glycolysis. Annual Review of Plant Biology 47:185-214.

Plaxton, W. C., and F. E. Podestá. 2006. The functional organization and control of plant respiration. Critical Reviews in Plant Sciences 25:159-198.

Plaxton, W. C., and H. T. Tran. 2011. Metabolic adaptations of phosphate-starved plants. Plant Physiology 156:1006-1015.

Pochon, X., and R. D. Gates. 2010. A new Symbiodinium clade (Dinophyceae) from soritid foraminifera in Hawai'i. Molecular Phylogenetics and Evolution 56:492-497.

Pochon, X., H. M. Putnam, and R. D. Gates. 2014. Multi-gene analysis of Symbiodinium dinoflagellates: a perspective on rarity, symbiosis, and evolution. Peerj 2:e394.

Porter, J. W., W. K. Fitt, H. J. Spero, C. S. Rogers, and M. W. White. 1989. Bleaching in reef corals: Physiological and stable isotopic responses. Proceedings of the National Academy of Sciences of the United States of America 86:9342-9346.

Prosser, C. L. 1991. Environmental and metabolism animal physiology.

Qian, Q., J. F. Curran, and G. R. Björk. 1998. The Methyl Group of theN 6-Methyl-N 6Threonylcarbamoyladenosine in tRNA of Escherichia coli Modestly Improves the Efficiency of the tRNA. Journal of Bacteriology 180:1808-1813.

Quigley, K. M., L. K. Bay, and B. L. Willis. 2018. Leveraging new knowledge of Symbiodinium community regulation in corals for conservation and reef restoration. Marine Ecology Progress Series 600:245-253.

Rabalais, N. N., R. E. Turner, R. J. Diaz, and D. Justić. 2009. Global change and eutrophication of coastal waters. ICES Journal of Marine Science 66:1528-1537.

Radi, R., A. Cassina, and R. Hodara. 2002. Nitric oxide and peroxynitrite interactions with mitochondria. Biological Chemistry 383:401-409.

Ragni, M., R. L. Airs, S. J. Hennige, D. J. Suggett, M. E. Warner, and R. J. Geider. 2010. PSII photoinhibition and photorepair in Symbiodinium (Pyrrhophyta) differs between thermally tolerant and sensitive phylotypes. Marine Ecology Progress Series 406:57-70.

Rands, M., B. Loughman, and A. Douglas. 1993. The symbiotic interface in an alga-invertebrate symbiosis. Proceedigns of the Royal Society of London. B 253:161-165.

Rasmusson, A. G., I. M. Møller, and J. M. Palmer. 1990. Component of the alternative oxidase localized to the matrix surface of the inner membrane of plant mitochondria. FEBS Letters 259:311-314.

Reaka-Kudla, M. L. 2001. Known and unknown biodiversity, risk of extinction and conservation strategy in the sea. Pages 19-33 Waters in peril. Springer.

Reaka-Kudla, M. L., D. E. Wilson, and E. O. Wilson. 1997. Biodiversity II: understanding and protecting our biological resources. Journal of Insect Conservation 1:247-250.

Rehman, A. U., M. Szabó, Z. Deák, L. Sass, A. Larkum, P. Ralph, and I. Vass. 2016. Symbiodinium sp. cells produce light-induced intra-and extracellular singlet oxygen, which mediates photodamage of the photosynthetic apparatus and has the potential to interact with the animal host in coral symbiosis. New Phytologist 212:472-484.

Reich, H. G., D. L. Robertson, and G. Goodbody-Gringley. 2017. Do the shuffle: Changes in Symbiodinium consortia throughout juvenile coral development. PLoS ONE 12:e0171768.

Reynolds, J. M., B. U. Bruns, W. K. Fitt, and G. W. Schmidt. 2008. Enhanced photoprotection pathways in symbiotic dinoflagellates of shallow-water corals and other cnidarians. Proceedings of the National Academy of Sciences 105:13674-13678. 
Richier, S., P. Furla, A. Plantivaux, P.-L. Merle, and D. Allemand. 2005. Symbiosis-induced adaptation to oxidative stress. Journal of Experimental Biology 208:277-285.

Roberts, J., P. Davies, L. Fixter, and T. Preston. 1999. Primary site and initial products of ammonium assimilation in the symbiotic sea anemone Anemonia viridis. Marine Biology 135:223-236.

Roberts, J., L. Fixter, and P. Davies. 2001. Ammonium metabolism in the symbiotic sea anemone Anemonia viridis. Hydrobiologia 461:25-35.

Roberty, S., B. Bailleul, N. Berne, F. Franck, and P. Cardol. 2014. PSI Mehler reaction is the main alternative photosynthetic electron pathway in Symbiodinium sp., symbiotic dinoflagellates of cnidarians. New Phytologist 204:81-91.

Robison, J. D., and M. E. Warner. 2006. Differential impacts of photoacclimation and thermal stress on the photobiology of four different phylotypes of Symbiodinium (pyrrhophyta) 1. Journal of Phycology 42:568-579.

Rohwer, F., M. Breitbart, J. Jara, F. Azam, and N. Knowlton. 2001. Diversity of bacteria associated with the Caribbean coral Montastraea franksi. Coral Reefs 20:85-91.

Ronimus, R. S., H. W. Morgan, and Y.-H. Ding. 1999. Phosphofructokinase activities within the order Spirochaetales and the characterisation of the pyrophosphate-dependent phosphofructokinase from Spirochaeta thermophila. Archives of Microbiology 172:401-406.

Rosic, N. N., M. Pernice, S. Dove, S. Dunn, and O. Hoegh-Guldberg. 2011. Gene expression profiles of cytosolic heat shock proteins Hsp70 and Hsp90 from symbiotic dinoflagellates in response to thermal stress: possible implications for coral bleaching. Cell Stress and Chaperones 16:69-80.

Rosset, S., C. D'Angelo, and J. Wiedenmann. 2015. Ultrastructural biomarkers in symbiotic algae reflect the availability of dissolved inorganic nutrients and particulate food to the reef coral holobiont. Frontiers in Marine Science 2:103.

Rosset, S., J. Wiedenmann, A. J. Reed, and C. D'Angelo. 2017. Phosphate deficiency promotes coral bleaching and is reflected by the ultrastructure of symbiotic dinoflagellates. Marine Pollution Bulletin 118: 180-187.

Rowan, R. 2004. Coral bleaching: thermal adaptation in reef coral symbionts. Nature 430:742.

Ruiz-Jones, L. J., and S. R. Palumbi. 2017. Tidal heat pulses on a reef trigger a fine-tuned transcriptional response in corals to maintain homeostasis. Science Advances 3:e1601298.

Rychter, A. M., and M. Mikulska. 1990. The relationship between phosphate status and cyanideresistant respiration in bean roots. Physiologia Plantarum 79:663-667.

Sachs, J. L., and T. P. Wilcox. 2006. A shift to parasitism in the jellyfish symbiont Symbiodinium microadriaticum. Proceedings of the Royal Society of London B: Biological Sciences 273:425-429.

Safaie, A., N. J. Silbiger, T. R. McClanahan, G. Pawlak, D. J. Barshis, J. L. Hench, J. S. Rogers, G. J. Williams, and K. A. Davis. 2018. High frequency temperature variability reduces the risk of coral bleaching. Nature Communications 9.

Santos, S., C. Gutierrez-Rodriguez, H. Lasker, and M. Coffroth. 2003. Symbiodinium sp. associations in the gorgonian Pseudopterogorgia elisabethae in the Bahamas: high levels of genetic variability and population structure in symbiotic dinoflagellates. Marine Biology 143:111-120.

Santos, S. R., and M. A. Coffroth. 2003. Molecular genetic evidence that dinoflagellates belonging to the genus Symbiodinium Freudenthal are haploid. The Biological Bulletin 204:10-20.

Saragosti, E., D. Tchernov, A. Katsir, and Y. Shaked. 2010. Extracellular production and degradation of superoxide in the coral Stylophora pistillata and cultured Symbiodinium. PLoS ONE 5:e12508.

Schaffelke, B., J. Carleton, M. Skuza, I. Zagorskis, and M. J. Furnas. 2012. Water quality in the inshore Great Barrier Reef lagoon: Implications for long-term monitoring and management. Marine Pollution Bulletin 65:249-260. 
Schreiber, U. 2004. Pulse-amplitude-modulation (PAM) fluorometry and saturation pulse method: an overview. Pages 279-319 Chlorophyll a fluorescence. Springer.

Shikanai, T. 2014. Central role of cyclic electron transport around photosystem I in the regulation of photosynthesis. Current Opinion in Biotechnology 26:25-30.

Shin, R., R. H. Berg, and D. P. Schachtman. 2005. Reactive oxygen species and root hairs in Arabidopsis root response to nitrogen, phosphorus and potassium deficiency. Plant and Cell Physiology 46:1350-1357.

Shinzato, C., E. Shoguchi, T. Kawashima, M. Hamada, K. Hisata, M. Tanaka, M. Fujie, M. Fujiwara, R. Koyanagi, and T. Ikuta. 2011. Using the Acropora digitifera genome to understand coral responses to environmental change. Nature 476:320.

Short, F. T., M. W. Davis, R. A. Gibson, and C. F. Zimmermann. 1985. Evidence for phosphorus limitation in carbonate sediments of the seagrass Syringodium filiforme. Estuarine, Coastal and Shelf Science 20:419-430.

Sieger, S. M., B. K. Kristensen, C. A. Robson, S. Amirsadeghi, E. W. Eng, A. Abdel-Mesih, I. M. Møller, and G. C. Vanlerberghe. 2005. The role of alternative oxidase in modulating carbon use efficiency and growth during macronutrient stress in tobacco cells. Journal of Experimental Botany 56:1499-1515.

Silverstein, R. N., A. M. Correa, and A. C. Baker. 2012. Specificity is rarely absolute in coral-algal symbiosis: implications for coral response to climate change. Proceedings of the Royal Society of London B: Biological Sciences 279:2609-2618.

Silverstein, R. N., A. M. Correa, T. C. LaJeunesse, and A. C. Baker. 2011. Novel algal symbiont (Symbiodinium spp.) diversity in reef corals of Western Australia. Marine Ecology Progress Series 422:63-75.

Silverstein, R. N., R. Cunning, and A. C. Baker. 2015. Change in algal symbiont communities after bleaching, not prior heat exposure, increases heat tolerance of reef corals. Global Change Biology 21:236-249.

Slavov, C., V. Schrameyer, M. Reus, P. J. Ralph, R. Hill, C. Büchel, A. W. Larkum, and A. R. Holzwarth. 2016. "Super-quenching" state protects Symbiodinium from thermal stressimplications for coral bleaching. Biochimica et Biophysica Acta (BBA)-Bioenergetics 1857:840-847.

Gustafsson, M., M. E. Baird, and P. J. Ralph. 2014. Modeling photoinhibition-driven bleaching in Scleractinian coral as a function of light, temperature, and heterotrophy. Limnology and Oceanography 59:603-622.

Smith, D. C., and A. E. Douglas. 1987. The biology of symbiosis. Edward Arnold (Publishers) Ltd.

Smith, D. J., D. J. Suggett, and N. R. Baker. 2005. Is photoinhibition of zooxanthellae photosynthesis the primary cause of thermal bleaching in corals? Global Change Biology 11:1-11.

Smith, G., and L. Muscatine. 1999. Cell cycle of symbiotic dinoflagellates: variation in G1 phaseduration with anemone nutritional status and macronutrient supply in the Aiptasia pulchellaSymbiodinium pulchrorum symbiosis. Marine Biology 134:405-418.

Snidvongs, A., and R. Kinzie. 1994. Effects of nitrogen and phosphorus enrichement on in vivo symbiotic zooxanthellae of Pocillopora damicornis. Marine Biology 118:705-711.

Snyder, C. M., E. H. Shroff, J. Liu, and N. S. Chandel. 2009. Nitric oxide induces cell death by regulating anti-apoptotic BCL-2 family members. PLoS ONE 4:e7059.

Stambler, N., N. Popper, Z. Dubinsky, and J. Stimson. 1991. Effects of nutrient enrichment and water motion on the coral Pocillopora damicornis. Pacific Science 45:299-307

Stanley, G. D., and P. K. Swart. 1995. Evolution of the coral-zooxanthellae symbiosis during the Triassic: a geochemical approach. Paleobiology 21:179-199.

Stanley Jr, G. D. 2006. Photosymbiosis and the evolution of modern coral reefs. Evolution 1:3.

Starzak, D. E., R. G. Quinnell, M. R. Nitschke, and S. K. J. M. b. Davy. 2014. The influence of symbiont type on photosynthetic carbon flux in a model cnidarian-dinoflagellate symbiosis. Marine Biology 161:711-724. 
Stat, M., and R. D. Gates. 2011. Clade D Symbiodinium in scleractinian corals: a "nugget" of hope, a selfish opportunist, an ominous sign, or all of the above? Journal of Marine Biology 2011.

Stat, M., D. M. Yost, and R. D. Gates. 2015. Geographic structure and host specificity shape the community composition of symbiotic dinoflagellates in corals from the Northwestern Hawaiian Islands. Coral Reefs 34:1075-1086.

Stimson, J., and R. A. Kinzie III. 1991. The temporal pattern and rate of release of zooxanthellae from the reef coral Pocillopora damicornis (Linnaeus) under nitrogen-enrichment and control conditions. Journal of Experimental Marine Biology and Ecology 153:63-74.

Stitt, M. 1990. Fructose-2, 6-bisphosphate as a regulatory molecule in plants. Annual Review of Plant Biology 41:153-185.

Stochaj, W. R., and A. R. Grossman. 1997. Differences in the protein profiles of cultured and endosymbiotic Symbiodinium sp.(Pyrrophyta) from the anemone Aiptasia pallida (Anthozoa). Journal of Phycology 33:44-53.

Swain, T. D., J. Chandler, V. Backman, and L. J. F. E. Marcelino. 2017. Consensus thermotolerance ranking for 110 Symbiodinium phylotypes: an exemplar utilization of a novel iterative partial-rank aggregation tool with broad application potential. Functional Ecology 31:172183.

Swanson, R., and O. Hoegh-Guldberg. 1998. Amino acid synthesis in the symbiotic sea anemone Aiptasia pulchella. Marine Biology 131:83-93.

Szabó, I., E. Bergantino, and G. M. Giacometti. 2005. Light and oxygenic photosynthesis: energy dissipation as a protection mechanism against photo-oxidation. EMBO Reports 6:629-634.

Szklarczyk, D., A. L. Gable, D. Lyon, A. Junge, S. Wyder, J. Huerta-Cepas, M. Simonovic, N. T. Doncheva, J. H. Morris, and P. Bork. 2018. STRING v11: protein-protein association networks with increased coverage, supporting functional discovery in genome-wide experimental datasets. Nucleic Acids Research 47:D607-D613.

Szmant, A. M. 2002. Nutrient enrichment on coral reefs: Is it a major cause of coral reef decline? Estuaries 25:743-766.

Takahashi, S., and M. R. Badger. 2011. Photoprotection in plants: a new light on photosystem II damage. Trends in Plant Science 16:53-60.

Takahashi, S., and N. Murata. 2005. Interruption of the Calvin cycle inhibits the repair of Photosystem II from photodamage. Biochim Biophys Acta 1708:352-361.

Takahashi, S., T. Nakamura, M. Sakamizu, R. v. Woesik, and H. Yamasaki. 2004. Repair machinery of symbiotic photosynthesis as the primary target of heat stress for reef-building corals. Plant and Cell Physiology 45:251-255.

Takahashi, S., S. Whitney, S. Itoh, T. Maruyama, and M. Badger. 2008. Heat stress causes inhibition of the de novo synthesis of antenna proteins and photobleaching in cultured Symbiodinium. Proceedings of the National Academy of Sciences 105:4203-4208.

Takahashi, S., S. M. Whitney, and M. R. Badger. 2009. Different thermal sensitivity of the repair of photodamaged photosynthetic machinery in cultured Symbiodinium species. Proceedings of the National Academy of Sciences 106:3237-3242.

Takahashi, S., M. Yoshioka-Nishimura, D. Nanba, and M. R. Badger. 2013. Thermal acclimation of the symbiotic alga Symbiodinium spp. alleviates photobleaching under heat stress. Plant Physiology 161:477-485.

Tanaka, Y., T. Miyajima, I. Koike, T. Hayashibara, and H. Ogawa. 2006. Translocation and conservation of organic nitrogen within the coral-zooxanthella symbiotic system of Acropora pulchra, as demonstrated by dual isotope-labeling techniques. Journal of Experimental Marine Biology and Ecology 336:110-119.

Tchernov, D., M. Y. Gorbunov, C. d. Vargas, S. N. Yadav, A. J. Milligan, M. Häggblom, and P. G. Falkowski. 2004. Membrane lipids of symbiotic algae are diagnostic of sensitivity to thermal bleaching in corals. PNAS 37: 13531-13535 
Tchernov, D., H. Kvitt, L. Haramaty, T. S. Bibby, M. Y. Gorbunov, H. Rosenfeld, and P. G. Falkowski. 2011. Apoptosis and the selective survival of host animals following thermal bleaching in zooxanthellate corals. Proceedings of the National Academy of Sciences 108:9905-9909.

Tewari, R. K., P. Kumar, and P. N. Sharma. 2007. Oxidative stress and antioxidant responses in young leaves of mulberry plants grown under nitrogen, phosphorus or potassium deficiency. Journal of Integrative Plant Biology 49:313-322.

Thornhill, D. J., T. C. LaJeunesse, D. W. Kemp, W. K. Fitt, and G. W. Schmidt. 2006. Multi-year, seasonal genotypic surveys of coral-algal symbioses reveal prevalent stability or postbleaching reversion. Marine Biology 148:711-722.

Thornhill, D. J., A. M. Lewis, D. C. Wham, and T. C. LaJeunesse. 2014. Host-specialist lineages dominate the adaptive radiation of reef coral endosymbionts. Evolution 68:352-367.

Thurber, R. L. V., D. E. Burkepile, C. Fuchs, A. A. Shantz, R. McMinds, and J. R. Zaneveld. 2014. Chronic nutrient enrichment increases prevalence and severity of coral disease and bleaching. Global Change Biology 20:544-554.

Tilman, D., J. Fargione, B. Wolff, C. D'antonio, A. Dobson, R. Howarth, D. Schindler, W. H. Schlesinger, D. Simberloff, and D. Swackhamer. 2001. Forecasting agriculturally driven global environmental change. Science 292:281-284.

Titlyanov, E., T. Titlyanova, T. Kalita, and I. Yakovleva. 2004. Rhythmicity in division and degradation of zooxanthellae in the hermatypic coral Stylophora pistillata. Symbiosis 36:211224.

Titlyanov, E., T. Titlyanova, V. Leletkin, J. Tsukahara, R. Van Woesik, and K. Yamazato. 1996. Degradation of zooxanthellae and regulation of their density in hermatypic corals. Marine Ecology Progress Series 139:167-178.

Tolleter, D., F. O. Seneca, J. C. DeNofrio, C. J. Krediet, S. R. Palumbi, J. R. Pringle, and A. R. Grossman. 2013. Coral Bleaching Independent of Photosynthetic Activity. Current Biology 23:1782-1786.

Tomascik, T., and F. Sander. 1987. Effects of eutrophication on reef-building corals. III. Reproduction of the reef-building coral Porites porites. Marine biology. Berlin, Heidelberg 94:77-94.

Torabi, S., M. Wissuwa, M. Heidari, M. R. Naghavi, K. Gilany, M. R. Hajirezaei, M. Omidi, B. Yazdi-Samadi, A. M. Ismail, and G. H. Salekdeh. 2009. A comparative proteome approach to decipher the mechanism of rice adaptation to phosphorous deficiency. Proteomics 9:159-170.

Trapido-Rosenthal, H. G., K. H. Sharp, T. S. Galloway, and C. E. Morrall. 2001. Nitric oxide and cnidarian-dinoflagellate symbioses: pieces of a puzzle. American Zoologist 41:247-257.

Tremblay, P., R. Grover, J. F. Maguer, L. Legendre, and C. Ferrier-Pagès. 2012. Autotrophic carbon budget in coral tissue: a new 13C-based model of photosynthate translocation. Journal of Experimental Biology 215:1384-1393.

Trench, R. 1997. Diversity of symbiotic dinoflagellates and the evolution of microalgal-invertebrate symbioses. Pages 1275-1286 in Proceedings of the 8th International Coral Reef Symposium.

Tyanova, S., T. Temu, P. Sinitcyn, A. Carlson, M. Y. Hein, T. Geiger, M. Mann, and J. Cox. 2016. The Perseus computational platform for comprehensive analysis of (prote) omics data. Nature Methods 13:731.

Tyers, M., and M. Mann. 2003. From genomics to proteomics. Nature 422:193.

Ulstrup, K. E., R. Hill, and P. J. Ralph. 2005. Photosynthetic impact of hypoxia on in hospite zooxanthellae in the scleractinian coralPocillopora damicornis. Marine Ecology Progress Series 286:125-132.

Valiela, I., C. Owens, E. Elmstrom, and J. Lloret. 2016. Eutrophication of Cape Cod estuaries: effect of decadal changes in global-driven atmospheric and local-scale wastewater nutrient loads. Marine Pollution Bulletin 110:309-315. 
Van Der Heijden, M. G., R. D. Bardgett, and N. M. Van Straalen. 2008. The unseen majority: soil microbes as drivers of plant diversity and productivity in terrestrial ecosystems. Ecology letters 11:296-310.

Van der Heijden, M. G., J. N. Klironomos, M. Ursic, P. Moutoglis, R. Streitwolf-Engel, T. Boller, A. Wiemken, and I. R. Sanders. 1998. Mycorrhizal fungal diversity determines plant biodiversity, ecosystem variability and productivity. Nature 396:69.

Van Hooidonk, R., J. Maynard, and S. Planes. 2013. Temporary refugia for coral reefs in a warming world. Nature Climate Change 3:508.

Van Mooy, B. A., H. F. Fredricks, B. E. Pedler, S. T. Dyhrman, D. M. Karl, M. Koblížek, M. W. Lomas, T. J. Mincer, L. R. Moore, and T. Moutin. 2009. Phytoplankton in the ocean use nonphosphorus lipids in response to phosphorus scarcity. Nature 458:69.

Van Oppen, M. 2001. In vitro establishment of symbiosis in Acropora millepora planulae. Coral Reefs 20:200-200.

Van Oppen, M. J., and R. D. Gates. 2006. Conservation genetics and the resilience of reef-building corals. Molecular Ecology 15:3863-3883.

Van Oppen, M. J., P. Souter, E. J. Howells, A. Heyward, and R. Berkelmans. 2011. Novel genetic diversity through somatic mutations: fuel for adaptation of reef corals? Diversity 3:405-423.

Venn, A., J. Loram, and A. Douglas. 2008. Photosynthetic symbioses in animals. Journal of Experimental Botany 59:1069-1080.

Veron, J., O. Hoegh-Guldberg, T. Lenton, J. Lough, D. Obura, P. Pearce-Kelly, C. Sheppard, M. Spalding, M. Stafford-Smith, and A. Rogers. 2009. The coral reef crisis: The critical importance of $<350$ ppm CO2. Marine Pollution Bulletin 58:1428-1436.

Vierling, E. 1991. The roles of heat shock proteins in plants. Annual review of plant biology 42:579620.

Vincent, J. B., and M. W. Crowder. 1995. Phosphatases in cell metabolism and signal transduction. Springer.

Visweswaraiah, J., S. Lageix, B. A. Castilho, L. Izotova, T. G. Kinzy, A. G. Hinnebusch, and E. Sattlegger. 2011. Evidence that eukaryotic translation elongation factor 1A (eEF1A) binds the Gcn 2 protein $C$ terminus and inhibits Gen2 activity. Journal of Biological Chemistry 286:36568-36579.

Vitova, M., K. Bisova, S. Kawano, and V. Zachleder. 2015. Accumulation of energy reserves in algae: from cell cycles to biotechnological applications. Biotechnology advances 33:12041218.

Wagner, D. E., P. Kramer, and R. Van Woesik. 2010. Species composition, habitat, and water quality influence coral bleaching in southern Florida. Marine Ecology Progress Series 408:65-78.

Wakefield, T. S., and S. C. Kempf. 2001. Development of host-and symbiont-specific monoclonal antibodies and confirmation of the origin of the symbiosome membrane in a cnidariandinoflagellate symbiosis. The Biological Bulletin 200:127-143.

Wang, J.-T., and A. E. Douglas. 1997. Nutrients, signals, and photosynthate release by symbiotic algae (the impact of taurine on the dinoflagellate alga Symbiodinium from the sea anemone Aiptasia pulchella). Plant Physiology 114:631-636.

Wang, J., and A. Douglas. 1999. Essential amino acid synthesis and nitrogen recycling in an algainvertebrate symbiosis. Marine Biology 135:219-222.

Wang, J., and A. E. Douglas. 1998. Nitrogen recycling or nitrogen conservation in an algainvertebrate symbiosis? Journal of Experimental Biology 201:2445-2453.

Wang, W., B. Vinocur, O. Shoseyov, and A. Altman. 2004. Role of plant heat-shock proteins and molecular chaperones in the abiotic stress response. Trends in plant science 9:244-252.

Wang, Y., and E. G. Ruby. 2011. The roles of NO in microbial symbioses. Cellular Microbiology 13:518-526. 
Warner, M., and S. Berry-Lowe. 2006. Differential xanthophyll cycling and photochemical activity in symbiotic dinoflagellates in multiple locations of three species of Caribbean coral. Journal of Experimental Marine Biology and Ecology 339:86-95.

Warner, M., W. Fitt, and G. Schmidt. 1996. The effects of elevated temperature on the photosynthetic efficiency of zooxanthellae in hospite from four different species of reef coral: a novel approach. Plant, Cell \& Environment 19:291-299.

Warner, M. E., W. K. Fitt, and G. W. Schmidt. 1999. Damage to photosystem II in symbiotic dinoflagellates: a determinant of coral bleaching. Proceedings of the National Academy of Sciences of the United States of America 96:8007-8012.

Warner, M. E., and D. J. Suggett. 2016. The photobiology of Symbiodinium spp.: linking physiological diversity to the implications of stress and resilience. Pages 489-509 The Cnidaria, Past, Present and Future. Springer.

Weber, M., D. De Beer, C. Lott, L. Polerecky, K. Kohls, R. M. Abed, T. G. Ferdelman, and K. E. Fabricius. 2012. Mechanisms of damage to corals exposed to sedimentation. Proceedings of the National Academy of Sciences 109:E1558-E1567.

Weis, V. M. 2008. Cellular mechanisms of Cnidarian bleaching: stress causes the collapse of symbiosis. Journal of Experimental Biology 211:3059-3066.

Whitehead, L., and A. Douglas. 2003. Metabolite comparisons and the identity of nutrients translocated from symbiotic algae to an animal host. Journal of Experimental Biology 206:3149-3157.

Whitney, S. M., and D. Yellowlees. 1995. Preliminary investigations into the structure and activity of ribulose bisphosphate carboxylase from two photosynthetic dinoflagellates. Journal of Phycology 31:138-146.

Wiedenmann, J., C. D'Angelo, E. G. Smith, A. N. Hunt, F. E. Legiret, A. D. Postle, and E. P. Achterberg. 2013. Nutrient enrichment can increase the susceptibility of reef corals to bleaching. Nature Climate Change 3:160-164.

Wietheger, A., P. L. Fisher, K. S. Gould, and S. K. Davy. 2015. Sensitivity to oxidative stress is not a definite predictor of thermal sensitivity in symbiotic dinoflagellates. Marine Biology 162:2067-2077.

Wilkerson, F., D. Kobayashi, and L. Muscatine. 1988. Mitotic index and size of symbiotic algae in Caribbean reef corals. Coral Reefs 7:29-36.

Wilkerson, F., and R. Trench. 1985. Nitrate assimilation by zooxanthellae maintained in laboratory culture. Marine Chemistry 16:385-393.

Wilkinson, T., and A. Douglas. 1995. Why pea aphids (Acyrthosiphon pisum) lacking symbiotic bacteria have elevated levels of the amino acid glutamine. Journal of Insect Physiology 41:921-927.

Wiśniewski, J. R., A. Zougman, N. Nagaraj, and M. Mann. 2009. Universal sample preparation method for proteome analysis. Nature methods 6:359.

Withers, K. J., A. J. Grant, and R. Hinde. 1998. Effects of free amino acids on the isolated symbiotic algae of the coral Plesiastrea versipora (Lamarck): absence of a host release factor response. Comparative Biochemistry and Physiology Part A: Molecular \& Integrative Physiology 120:599-607.

Wojtera-Kwiczor, J., F. Groß, H.-M. Leffers, M. Kang, M. Schneider, and R. Scheibe. 2013. Transfer of a redox-signal through the cytosol by redox-dependent microcompartmentation of glycolytic enzymes at mitochondria and actin cytoskeleton. Frontiers in Plant Science 3:284.

Wooldridge, S. A. 2009. Water quality and coral bleaching thresholds: Formalising the linkage for the inshore reefs of the Great Barrier Reef, Australia. Marine Pollution Bulletin 58:745-751.

Wooldridge, S. A. 2013. Breakdown of the coral-algae symbiosis: towards formalising a linkage between warm-water bleaching thresholds and the growth rate of the intracellular zooxanthellae. Biogeosciences 10:1647-1658. 
Wooldridge, S. A. 2016. Excess seawater nutrients, enlarged algal symbiont densities and bleaching sensitive reef locations: 1. Identifying thresholds of concern for the Great Barrier Reef, Australia. Marine Pollution Bulletin.

Wooldridge, S. A., S. F. Heron, J. E. Brodie, T. J. Done, I. Masiri, and S. Hinrichs. 2017. Excess seawater nutrients, enlarged algal symbiont densities and bleaching sensitive reef locations: 2 . A regional-scale predictive model for the Great Barrier Reef, Australia. Marine Pollution Bulletin 114:343-354.

Yahalom, A., T. H. Kim, B. Roy, R. Singer, A. G. Von Arnim, and D. A. Chamovitz. 2008. Arabidopsis eIF3e is regulated by the COP9 signalosome and has an impact on development and protein translation. The Plant Journal 53:300-311.

Yángüez, E., A. B. Castro-Sanz, N. Fernandez-Bautista, J. C. Oliveros, and M. M. Castellano. 2013. Analysis of genome-wide changes in the translatome of Arabidopsis seedlings subjected to heat stress. PLoS ONE 8:e71425.

Yellowlees, D., T. A. V. Rees, and W. Leggat. 2008. Metabolic interactions between algal symbionts and invertebrate hosts. Plant, Cell \& Environment 31:679-694.

Young, A. J. 1991. The photoprotective role of carotenoids in higher plants. Physiologia Plantarum 83:702-708.

Yu, T.-S., H. Kofler, R. E. Häusler, D. Hille, U.-I. Flügge, S. C. Zeeman, A. M. Smith, J. Kossmann, J. Lloyd, and G. Ritte. 2001. The Arabidopsis sex1 mutant is defective in the R1 protein, a general regulator of starch degradation in plants, and not in the chloroplast hexose transporter. The Plant Cell 13:1907-1918.

Zhang, K., H. Liu, P. Tao, and H. Chen. 2014. Comparative proteomic analyses provide new insights into low phosphorus stress responses in maize leaves. PLoS ONE 9:e98215.

Zoschke, R., Y. Qu, Y. O. Zubo, T. Börner, and C. Schmitz-Linneweber. 2013. Mutation of the pentatricopeptide repeat-SMR protein SVR7 impairs accumulation and translation of chloroplast ATP synthase subunits in Arabidopsis thaliana. Journal of plant research 126:403414. 


\section{Appendices}

\section{Appendix 1: Appendices for Chapter 2}

\section{A1.1. Confocal microscope images of stained lipid droplets and starch granules}

It was the intention to investigate the accumulation of both starch and lipid bodies under different temperature treatments using the high through put IN Cell analyser $6500 \mathrm{HS}$ microscope confocal microscope. However, the analysis of starch and lipid bodies was unable to be optimised in the time frame of this study due to equipment failure. Images of cells were however taken on the regular Olympus FV1000 confocal microscope (see figure below), to test and optimise cell staining in case the high through put microscope was replaced before the end of my thesis.

\section{Staining protocol:}

Symbiodiniaceae cells were suspended in $75 \%$ ethanol and put under high light conditions (PAR = $40 \mu \mathrm{mol}$ photons $\mathrm{m}^{-2} \mathrm{~s}^{-1}$ ) for $24 \mathrm{hrs}$ to bleach chlorophyll from cells. Cells were then palletised, the ethanol was decanted off and the cells (approximately 2 million) were suspended in artificial salt water (ASW).

\section{Staining lipid droplets}

$2 \mu 1$ of Nile red stock solution $(0.5 \mathrm{mg} / \mathrm{ml}$ in DMSO) was added to a $1 \mathrm{ml}$ suspension of cells, vortexed and left to sit for 30 minutes. After this time the cells were palletised, the solution was decanted off and the pellet was re-suspended in $500 \mu 1$ of ASW. This process was performed in a dark room to prevent the nile red stain from bleaching.

Staining of starch granules

2ul of DMSO and 8ul of lugols stain (2.5gs of potassium and $5 \mathrm{gs}$ of potassium iodine dissolved in $50 \mathrm{mls}$ of distilled water) was added to a $1 \mathrm{ml}$ suspension of cells, vortexed and left to sit for 30 minutes. After this time the cells were palletised, the solution was decanted off and the pellet was resuspended in 500ul of ASW.

\section{Control cells}

$2 \mathrm{ul}$ of DMSO was added to a $1 \mathrm{ml}$ suspension of cells, vortexed and left to sit for 30 minutes. After this time the cells were palletised, the solution was decanted off and the pellet was re-suspended in 500ul of ASW.

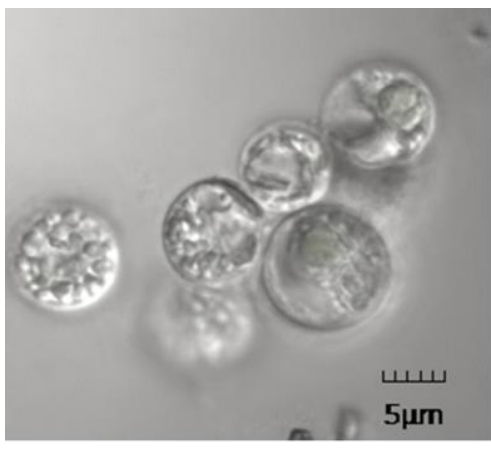

Control

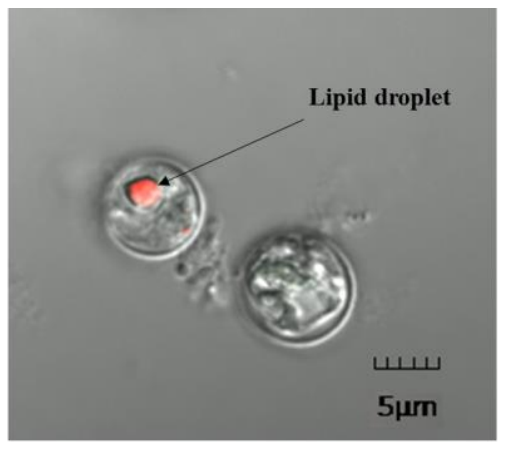

Nile red stain

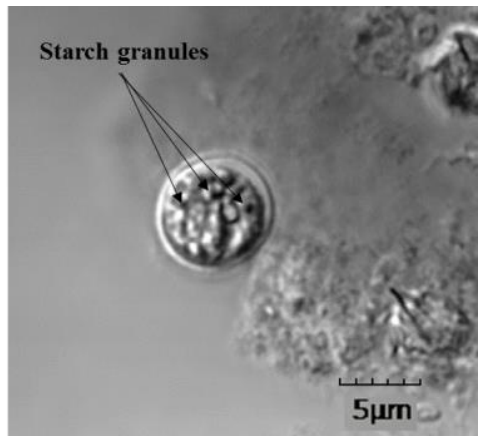

Lugols stain

Figure illustrating the staining of lipid droplets with Nile red stain and starch granules with Lugols stain in FlAp2 cells. Images were taken using the Differential interference contrast (DIC) channel and the 488nm and 568nm laser 


\section{A1.2. Statistical tests for chapter two physiological measurements}

\section{Chlorophyll fluorescence statistical tests}

Multiple Unpaired two tailed T-test results comparing effective quantum yields of control NZ01 cultures and those exposed to a rapid temperature increase daily

\begin{tabular}{|c|c|c|c|}
\hline $\begin{array}{c}\text { Experimental } \\
\text { day }\end{array}$ & t-ratio & df & p-value \\
\hline 8 & 1.774 & 26 & $\mathbf{0 . 4 7 4}$ \\
\hline 9 & 1.968 & 26 & $\mathbf{0 . 4 2 6}$ \\
\hline 10 & 1.722 & 26 & $\mathbf{0 . 4 7 4}$ \\
\hline 11 & 1.615 & 26 & $\mathbf{0 . 4 7 4}$ \\
\hline 12 & 0.166 & 26 & $\mathbf{0 . 9 5 2}$ \\
\hline 13 & 0.282 & 26 & $\mathbf{0 . 9 5 2}$ \\
\hline 14 & 0.579 & 26 & $\mathbf{0 . 9 1 9}$ \\
\hline 15 & 3.279 & 26 & $\mathbf{0 . 0 3 5}$ \\
\hline 16 & 1.841 & 26 & $\mathbf{0 . 4 7 4}$ \\
\hline 17 & 2.297 & 26 & $\mathbf{0 . 2 8 4}$ \\
\hline 18 & 1.045 & 26 & $\mathbf{0 . 7 6 7}$ \\
\hline 19 & 3.615 & 26 & $\mathbf{0 . 0 1 7}$ \\
\hline 20 & 3.721 & 26 & $\mathbf{0 . 0 1 4}$ \\
\hline 21 & 2.194 & 26 & $\mathbf{0 . 3 1 7}$ \\
\hline 22 & 3.559 & 26 & $\mathbf{0 . 0 1 8 8}$ \\
\hline 23 & 8.159 & 26 & $<\mathbf{0 . 0 0 1}$ \\
\hline 24 & 8.366 & 26 & $<\mathbf{0 . 0 0 1}$ \\
\hline
\end{tabular}

Multiple Unpaired two tailed T-test results comparing maximum quantum yields of control NZ01 cultures and those exposed to a rapid temperature increase daily

\begin{tabular}{|c|c|c|c|}
\hline $\begin{array}{c}\text { Experimental } \\
\text { day }\end{array}$ & t-ratio & df & $\begin{array}{c}\text { p- } \\
\text { value }\end{array}$ \\
\hline 8 & 0.964 & 26 & $\mathbf{0 . 9 6 1}$ \\
\hline 9 & 0.929 & 26 & $\mathbf{0 . 9 6 1}$ \\
\hline 10 & 1.399 & 26 & $\mathbf{0 . 8 5 2}$ \\
\hline 11 & 0.066 & 26 & $\mathbf{0 . 9 6 1}$ \\
\hline 12 & 1.008 & 26 & $\mathbf{0 . 9 6 1}$ \\
\hline
\end{tabular}




\begin{tabular}{|c|c|c|c|}
\hline 13 & 0.430 & 26 & $\mathbf{0 . 9 6 1}$ \\
\hline 14 & 1.052 & 26 & $\mathbf{0 . 9 6 1}$ \\
\hline 15 & 4.823 & 26 & $\mathbf{0 . 0 0 1}$ \\
\hline 16 & 0.860 & 26 & $\mathbf{0 . 9 6 1}$ \\
\hline 17 & 2.496 & 26 & $\mathbf{0 . 2 2 3}$ \\
\hline 18 & 1.556 & 26 & $\mathbf{0 . 7 8 9}$ \\
\hline 19 & 0.738 & 26 & $\mathbf{0 . 9 6 1}$ \\
\hline 20 & 0.675 & 26 & $\mathbf{0 . 9 6 1}$ \\
\hline 21 & 1.833 & 26 & $\mathbf{0 . 6 2 4}$ \\
\hline 22 & 5.990 & 26 & $\mathbf{4 . 0 6 1}$ \\
\hline 23 & 6.012 & 26 & $<\mathbf{0 . 0 0 1}$ \\
\hline 24 & 2.542 & 26 & $\mathbf{0 . 2 1 7}$ \\
\hline & & &
\end{tabular}

Results of a repeated measures ANOVA comparing how the effective quantum yields of NZO1 control cultures and those exposed to a slow temperature increase change over time

\begin{tabular}{|c|c|c|c|c|c|}
\hline Variation & df & Sum of squares & Mean square & F & p-value \\
\hline Time & 10 & 0.049 & 0.005 & $\mathbf{7 . 2 3 2}$ & $<\mathbf{0 . 0 0 1}$ \\
\hline Treatment & 1 & 0.225 & 0.225 & $\mathbf{1 8 9 . 0}$ & $<\mathbf{0 . 0 0 1}$ \\
\hline Interaction & 10 & 0.055 & 0.005 & $\mathbf{7 . 5 5 9}$ & $<\mathbf{0 . 0 0 1}$ \\
\hline Residual & 130 & 0.094 & 0.001 & & \\
\hline Total & $\mathbf{1 5 1}$ & & & & \\
\hline
\end{tabular}

Dunnet's multiple comparison test comparing the effective quantum yield of NZ01 cultures averaged over the first six days of the active experimental period (when all cultures including the treatments were at $25^{\circ} \mathrm{C}$ ), with the yields on days 15-24. Due to the interaction of the repeated measures ANOVA as seen in the table above these comparisons were performed separately for the control and treatment cultures.

\begin{tabular}{|c|c|c|c|}
\hline Comparison & $\begin{array}{c}\text { Mean } \\
\text { difference }\end{array}$ & $\begin{array}{c}\mathbf{9 5 \%} \text { confidence } \\
\text { interval of difference }\end{array}$ & Adjusted p-value \\
\hline Control cultures & & & \\
\hline Average vs. day 15 & $-6.629 \mathrm{e}^{-5}$ & -0.028 to 0.028 & $>\mathbf{0 . 9 9 9 9}$ \\
\hline Average vs. day 16 & 0.007 & -0.021 to 0.035 & $\mathbf{0 . 9 9 2 7}$ \\
\hline Average vs. day 17 & -0.004 & -0.032 to 0.024 & $\mathbf{0 . 9 9 9}$ \\
\hline Average vs. day 18 & 0.037 & 0.0087 to 0.065 & $\mathbf{0 . 0 0 4}$ \\
\hline Average vs. day 19 & -0.017 & -0.04542 to 0.0106 & $\mathbf{0 . 4 4 4}$ \\
\hline
\end{tabular}




\begin{tabular}{|l|l|l|l|}
\hline Average vs. day 20 & -0.025 & -0.0515 to 0.004 & $\mathbf{0 . 1 4 4}$ \\
\hline Average vs. day 21 & -0.029 & -0.057 to -0.001 & $\mathbf{0 . 0 3 9}$ \\
\hline Average vs. day 22 & -0.032 & -0.060 to -0.004 & $\mathbf{0 . 0 1 8}$ \\
\hline Average vs. day 23 & -0.041 & -0.069 to -0.013 & $\mathbf{0 . 0 0 1}$ \\
\hline Average vs. day 24 & -0.031 & -0.059 to -0.003 & $\mathbf{0 . 0 2 3}$ \\
\hline Treatment cultures & & & $\mathbf{0 . 0 2 8}$ \\
\hline Average vs. day 15 & 0.030 & 0.002 to 0.058 & $\mathbf{0 . 9 9 9}$ \\
\hline Average vs. day 16 & 0.005 & -0.023 to 0.033 & $\mathbf{0 . 9 9 9}$ \\
\hline Average vs. day 17 & -0.002 & -0.030 to 0.026 & $\mathbf{0 . 3 0 7}$ \\
\hline Average vs. day 18 & -0.020 & -0.048 to 0.008 & $\mathbf{0 . 9 9 9}$ \\
\hline Average vs. day 19 & 0.004 & -0.024 to 0.032 & $\mathbf{0 . 8 9 0}$ \\
\hline Average vs. day 20 & -0.011 & -0.039 to 0.017 & $\mathbf{0 . 9 9 9}$ \\
\hline Average vs. day 21 & -0.005 & -0.03 to 0.023 & $\mathbf{0 . 4 7 3}$ \\
\hline Average vs. day 22 & 0.017 & -0.011 to 0.045 & $\mathbf{0 . 9 9 9}$ \\
\hline Average vs. day 23 & -0.001 & -0.029 to 0.027 & $\mathbf{0 . 1 5 9}$ \\
\hline Average vs. day 24 & -0.024 & -0.051 to 0.005 & \\
\hline
\end{tabular}

Multiple Unpaired two tailed T-test results comparing maximum quantum yields of control NZ01 cultures and those exposed to a slow temperature increase daily

\begin{tabular}{|c|c|c|c|}
\hline $\begin{array}{c}\text { Experimental } \\
\text { day }\end{array}$ & t-ratio & df & p-value \\
\hline 8 & 2.097 & 26 & $\mathbf{0 . 0 4 3}$ \\
\hline 9 & 3.082 & 26 & $\mathbf{0 . 0 4 7}$ \\
\hline 10 & 1.696 & 26 & $\mathbf{0 . 3 2 9}$ \\
\hline 11 & 1.734 & 26 & $\mathbf{0 . 3 2 9}$ \\
\hline 12 & 0.272 & 26 & $\mathbf{0 . 7 8 8}$ \\
\hline 13 & 0.857 & 26 & $\mathbf{0 . 6 3 9}$ \\
\hline 14 & 2.699 & 26 & $\mathbf{0 . 0 9 2}$ \\
\hline 15 & 5.652 & 26 & $<\mathbf{0 . 0 0 1}$ \\
\hline 16 & 3.031 & 26 & $\mathbf{0 . 0 4 8}$ \\
\hline 17 & 2.114 & 26 & $\mathbf{0 . 2 0 3}$ \\
\hline 18 & 7.357 & 26 & $<\mathbf{0 . 0 0 1}$ \\
\hline 19 & 2.385 & 26 & $\mathbf{0 . 1 6 0}$ \\
\hline
\end{tabular}




\begin{tabular}{|l|l|c|c|}
\hline 20 & 4.078 & 26 & $\mathbf{0 . 0 0 5}$ \\
\hline 21 & 2.214 & 26 & $\mathbf{0 . 1 9 7}$ \\
\hline 22 & 8.625 & 26 & $<\mathbf{0 . 0 0 1}$ \\
\hline 23 & 8.506 & 26 & $<\mathbf{0 . 0 0 1}$ \\
\hline 24 & 3.241 & 26 & $\mathbf{0 . 0 3 5}$ \\
\hline
\end{tabular}

Multiple Unpaired two tailed T-test results comparing effective quantum yields of control FlAp2 cultures and those exposed to a rapid temperature increase daily

\begin{tabular}{|c|c|c|c|}
\hline $\begin{array}{c}\text { Experimental } \\
\text { day }\end{array}$ & t-ratio & df & p-value \\
\hline 8 & 1.200 & 30 & 0.422 \\
\hline 9 & 3.107 & 30 & 0.032 \\
\hline 10 & 0.666 & 30 & 0.510 \\
\hline 11 & 2.451 & 30 & 0.116 \\
\hline 12 & 10.32 & 30 & $<0.001$ \\
\hline 13 & 5.592 & 30 & $<0.001$ \\
\hline 14 & 1.695 & 30 & 0.294 \\
\hline 15 & 6.052 & 30 & $<0.001$ \\
\hline 16 & 3.025 & 29 & 0.036 \\
\hline 17 & 1.899 & 30 & 0.294 \\
\hline 18 & 8.145 & 30 & $<0.001$ \\
\hline 19 & 1.880 & 30 & 0.293 \\
\hline 20 & 3.419 & 30 & 0.016 \\
\hline 21 & 4.417 & 30 & 0.001 \\
\hline 22 & 9.016 & 30 & $<0.001$ \\
\hline 23 & 15.13 & 30 & $<0.001$ \\
\hline
\end{tabular}

Multiple Unpaired two tailed T-test results comparing maximum quantum yields of control FlAp2 cultures and those exposed to a rapid temperature increase daily

\begin{tabular}{|c|c|c|c|}
\hline $\begin{array}{c}\text { Experimental } \\
\text { day }\end{array}$ & t-ratio & df & p-value \\
\hline 8 & 0.429 & 30 & $\mathbf{0 . 9 6 4 3}$ \\
\hline 9 & 2.644 & 30 & $\mathbf{0 . 1 3 3}$ \\
\hline 10 & 0.327 & 30 & $\mathbf{0 . 9 6 4}$ \\
\hline
\end{tabular}




\begin{tabular}{|l|l|l|c|}
\hline 11 & 6.187 & 30 & $<\mathbf{0 . 0 0 1}$ \\
\hline 12 & 1.224 & 29 & $\mathbf{0 . 8 2 8}$ \\
\hline 13 & 1.340 & 29 & $\mathbf{0 . 8 1 6}$ \\
\hline 14 & 1.456 & 29 & $\mathbf{0 . 7 8 3}$ \\
\hline 15 & 1.603 & 30 & $\mathbf{0 . 7 1 9}$ \\
\hline 16 & 1.245 & 30 & $\mathbf{0 . 8 2 8}$ \\
\hline 17 & 3.23 & 30 & $\mathbf{0 . 0 3 8}$ \\
\hline 18 & 0.771 & 30 & $\mathbf{0 . 9 0 6}$ \\
\hline 19 & 0.289 & 30 & $\mathbf{0 . 9 6 4}$ \\
\hline 20 & 2.986 & 30 & $\mathbf{0 . 0 6 5}$ \\
\hline 21 & 1.140 & 30 & $\mathbf{0 . 8 2 8}$ \\
\hline 22 & 16.28 & 30 & $<\mathbf{0 . 0 0 1}$ \\
\hline 23 & 19.80 & 30 & $<\mathbf{0 . 0 0 1}$ \\
\hline
\end{tabular}

Multiple Unpaired two tailed T-test results comparing effective quantum yields of control FlAp2 cultures and those exposed to a slow temperature increase daily

\begin{tabular}{|c|c|c|c|}
\hline $\begin{array}{l}\text { Experimental } \\
\text { day }\end{array}$ & t-ratio & df & p-value \\
\hline 8 & 2.038 & 30 & 0.187 \\
\hline 9 & 4.146 & 30 & 0.002 \\
\hline 10 & 0.210 & 30 & 0.835 \\
\hline 11 & 4.339 & 30 & 0.001 \\
\hline 12 & 2.507 & 30 & 0.086 \\
\hline 13 & 5.787 & 30 & $<0.001$ \\
\hline 14 & 1.705 & 30 & 0.267 \\
\hline 15 & 1.596 & 30 & 0.267 \\
\hline 16 & 2.864 & 30 & 0.045 \\
\hline 17 & 4.064 & 30 & 0.002 \\
\hline 18 & 9.856 & 30 & $<0.001$ \\
\hline 19 & 9.200 & 30 & $<0.001$ \\
\hline 20 & 8.746 & 30 & $<0.001$ \\
\hline 21 & 13.75 & 30 & $<0.001$ \\
\hline 22 & 21.69 & 30 & $<0.001$ \\
\hline 23 & 33.25 & 30 & $<0.001$ \\
\hline
\end{tabular}


Multiple Unpaired two tailed T-test results comparing maximum quantum yields of control FlAp2 cultures and those exposed to a slow temperature increase daily

\begin{tabular}{|c|c|c|c|}
\hline $\begin{array}{c}\text { Experimental } \\
\text { day }\end{array}$ & t-ratio & df & p-value \\
\hline 8 & 1.707 & 29 & $\mathbf{0 . 5 1 6}$ \\
\hline 9 & 1.992 & 30 & $\mathbf{0 . 3 9 9}$ \\
\hline 10 & 1.232 & 29 & $\mathbf{0 . 5 9 1}$ \\
\hline 11 & 1.331 & 29 & $\mathbf{0 . 5 9 1}$ \\
\hline 12 & 1.429 & 29 & $\mathbf{0 . 5 9 1}$ \\
\hline 13 & 0.318 & 29 & $\mathbf{0 . 9 3 9}$ \\
\hline 14 & 0.020 & 28 & $\mathbf{0 . 9 8 4}$ \\
\hline 15 & 1.598 & 30 & $\mathbf{0 . 5 3 7}$ \\
\hline 16 & 1.997 & 30 & $\mathbf{0 . 3 9 9}$ \\
\hline 17 & 5.434 & 30 & $<\mathbf{0 . 0 0 1}$ \\
\hline 18 & 5.641 & 30 & $<\mathbf{0 . 0 0 1}$ \\
\hline 19 & 12.83 & 30 & $<\mathbf{0 . 0 0 1}$ \\
\hline 20 & 7.608 & 29 & $<\mathbf{0 . 0 0 1}$ \\
\hline 21 & 12.9 & 30 & $<\mathbf{0 . 0 0 1}$ \\
\hline 22 & 25.14 & 30 & $<0.001$ \\
\hline 23 & 46.55 & 28 & \\
\hline & & & $\mathbf{0 . 0 0 1}$ \\
\hline
\end{tabular}




\section{Chlorophyll a concentration}

ANOVA results comparing the chlorophyll a concentration of NZ01 cultures exposed to control temperatures, a slow or rapid temperature increase.

\begin{tabular}{|c|c|c|c|c|c|}
\hline Variation & df & Sum of squares & Mean square & F & p-value \\
\hline Treatment & $\mathbf{2}$ & 0.017 & 0.009 & $\mathbf{3 . 5 2 8}$ & $\mathbf{0 . 0 9 7}$ \\
\hline Residuals & $\mathbf{6}$ & 0.015 & 0.002 & & \\
\hline Total & $\mathbf{8}$ & 0.032 & & & \\
\hline
\end{tabular}

ANOVA results comparing the chlorophyll a concentration of FlAp2 cultures exposed to control temperatures, a slow or rapid temperature increase.

\begin{tabular}{|c|c|c|c|c|c|}
\hline Variation & df & Sum of squares & Mean square & F & p-value \\
\hline Treatment & $\mathbf{2}$ & 0.005 & 0.002 & $\mathbf{0 . 4 2 1}$ & $\mathbf{0 . 6 6 9}$ \\
\hline Residuals & $\mathbf{9}$ & 0.050 & 0.006 & & \\
\hline Total & $\mathbf{1 1}$ & 0.054 & & & \\
\hline
\end{tabular}

\section{Carotenoid concentration}

ANOVA results comparing the carotenoid concentration of NZ01 cultures exposed to control temperatures, a slow or rapid temperature increase.

\begin{tabular}{|c|c|c|c|c|c|}
\hline Variation & df & Sum of squares & Mean square & F & p-value \\
\hline Treatment & $\mathbf{2}$ & 0.046 & 0.023 & $\mathbf{6 . 3 8 8}$ & $\mathbf{0 . 0 3 3}$ \\
\hline Residuals & $\mathbf{6}$ & 0.022 & 0.004 & & \\
\hline Total & $\mathbf{8}$ & 0.068 & & & \\
\hline
\end{tabular}

Tukeys post hoc comparing the carotenoid concentration of NZ01 cultures exposed to control temperatures, a slow or rapid temperature increase

\begin{tabular}{|c|c|c|c|c|}
\hline Comparisons & df & Mean difference & $\mathbf{q}$ & p-value \\
\hline Control vs. Slow temperature increase & $\mathbf{6}$ & -0.040 & 1.149 & $\mathbf{0 . 7 1 0}$ \\
\hline Control vs. Rapid temperature increase & $\mathbf{6}$ & -0.168 & 4.837 & $\mathbf{0 . 0 3 3}$ \\
\hline $\begin{array}{c}\text { Slow temperature increase vs. Rapid } \\
\text { temperature increase }\end{array}$ & $\mathbf{6}$ & -0.128 & 3.689 & $\mathbf{0 . 0 8 9}$ \\
\hline
\end{tabular}


ANOVA results comparing the carotenoid concentration of FlAp2 cultures exposed to control temperatures, a slow or rapid temperature increase.

\begin{tabular}{|c|c|c|c|c|c|}
\hline Variation & df & Sum of squares & Mean square & F & p-value \\
\hline Treatment & $\mathbf{2}$ & 0.049 & 0.025 & $\mathbf{2 . 9 8 9}$ & $\mathbf{0 . 1 0 1}$ \\
\hline Residuals & $\mathbf{9}$ & 0.074 & 0.008 & & \\
\hline Total & $\mathbf{1 1}$ & 0.123 & & & \\
\hline
\end{tabular}

\section{Carotenoid to chlorophyll a concentration ratio}

ANOVA results comparing the ratio of carotenoids to chlorophyll a concentration of NZ01 cultures exposed to control temperatures, a slow or rapid temperature increase

\begin{tabular}{|c|c|c|c|c|c|}
\hline Variation & df & Sum of squares & Mean square & F & p-value \\
\hline Treatment & $\mathbf{2}$ & 0.814 & 0.407 & $\mathbf{0 . 2 6 1}$ & $\mathbf{0 . 7 7 8}$ \\
\hline Residuals & $\mathbf{6}$ & 9.353 & 1.559 & & \\
\hline Total & $\mathbf{8}$ & 10.17 & & & \\
\hline
\end{tabular}

ANOVA results comparing the ratio of carotenoids to chlorophyll a concentration of FlAp2 cultures exposed to control temperatures, a slow or rapid temperature increase.

\begin{tabular}{|c|c|c|c|c|c|}
\hline Variation & df & Sum of squares & Mean square & F & p-value \\
\hline Treatment & $\mathbf{2}$ & 2.216 & 1.108 & $\mathbf{0 . 8 5 0}$ & $\mathbf{0 . 4 6 3}$ \\
\hline Residuals & $\mathbf{8}$ & 10.43 & 1.304 & & \\
\hline Total & $\mathbf{1 0}$ & 12.64 & & & \\
\hline
\end{tabular}

\section{Respiratory and photosynthetic Oxygen flux}

\section{Gross photosynthesis}

ANOVA results comparing gross photosynthesis rates of NZO1 cultures exposed to control temperatures, a slow or rapid temperature increase

\begin{tabular}{|c|c|c|c|c|c|}
\hline Variation & df & Sum of squares & Mean square & F & p-value \\
\hline Treatment & $\mathbf{2}$ & $2.760 \mathrm{e}^{-06}$ & $1.377 \mathrm{e}^{-06}$ & $\mathbf{6 2 . 8 6}$ & $<\mathbf{0 . 0 0 1}$ \\
\hline Residuals & $\mathbf{8}$ & $1.972 \mathrm{e}^{-07}$ & $2.191 \mathrm{e}^{-08}$ & & \\
\hline Total & $\mathbf{1 0}$ & $2.952 \mathrm{e}^{-06}$ & & & \\
\hline
\end{tabular}


Tukeys post hoc comparing gross photosynthesis rates of NZ01 cultures exposed to control temperatures, a slow or rapid temperature increase

\begin{tabular}{|c|c|c|c|c|}
\hline Comparisons & df & Mean difference & $\mathbf{q}$ & p-value \\
\hline Control vs. Slow temperature increase & $\mathbf{9}$ & $2.956 \mathrm{e}^{-5}$ & 0.399 & $\mathbf{0 . 9 5 7}$ \\
\hline Control vs. Rapid temperature increase & $\mathbf{9}$ & -0.001 & 13.53 & $<\mathbf{0 . 0 0 1}$ \\
\hline $\begin{array}{c}\text { Slow temperature increase vs. Rapid } \\
\text { temperature increase }\end{array}$ & $\mathbf{9}$ & -0.001 & 13.93 & $<\mathbf{0 . 0 0 1}$ \\
\hline
\end{tabular}

ANOVA results comparing gross photosynthesis rates of FlAp2 cultures exposed to control temperatures, a slow or rapid temperature increase

\begin{tabular}{|c|c|c|c|c|c|}
\hline Variation & df & Sum of squares & Mean square & F & p-value \\
\hline Treatment & $\mathbf{2}$ & 0.617 & 0.309 & $\mathbf{3 0 . 3 0}$ & $<\mathbf{0 . 0 0 1}$ \\
\hline Residuals & $\mathbf{8}$ & 0.081 & 0.010 & & \\
\hline Total & $\mathbf{1 0}$ & 0.698 & & & \\
\hline
\end{tabular}

Tukeys post hoc comparing gross photosynthesis rates of Flap2 cultures exposed to control temperatures, a slow or rapid temperature increase

\begin{tabular}{|c|c|c|c|c|}
\hline Comparisons & df & Mean difference & $\mathbf{q}$ & p-value \\
\hline Control vs. Slow temperature increase & $\mathbf{8}$ & 0.556 & 11.01 & $<\mathbf{0 . 0 0 1}$ \\
\hline Control vs. Rapid temperature increase & $\mathbf{8}$ & 0.268 & 4.915 & $\mathbf{0 . 0 2 0}$ \\
\hline $\begin{array}{c}\text { Slow temperature increase vs. Rapid } \\
\text { temperature increase }\end{array}$ & $\mathbf{8}$ & -0.288 & 5.277 & $\mathbf{0 . 0 1 4}$ \\
\hline
\end{tabular}

\section{Respiration}

ANOVA results comparing respiration rates of NZ01 cultures exposed to control temperatures, a slow or rapid temperature increase.

\begin{tabular}{|c|c|c|c|c|c|}
\hline Variation & df & Sum of squares & Mean square & F & p-value \\
\hline Treatment & $\mathbf{2}$ & $5.406 \mathrm{e}^{-07}$ & $2.703 \mathrm{e}^{-07}$ & $\mathbf{1 3 2 . 6}$ & $<\mathbf{0 . 0 0 1}$ \\
\hline Residuals & $\mathbf{9}$ & $1.835 \mathrm{e}^{-08}$ & $2.039 \mathrm{e}^{-09}$ & & \\
\hline Total & $\mathbf{1 1}$ & $5.590 \mathrm{e}^{-07}$ & & & \\
\hline
\end{tabular}

Tukeys post hoc comparing respiration rates of NZ01 cultures exposed to control temperatures, a slow or rapid temperature increase

\begin{tabular}{|c|c|c|c|c|}
\hline Comparisons & df & Mean difference & $\mathbf{q}$ & p-value \\
\hline Control vs. Slow temperature increase & $\mathbf{9}$ & $6.592 \mathrm{e}^{-06}$ & 0.292 & $\mathbf{0 . 9 7 7}$ \\
\hline Control vs. Rapid temperature increase & $\mathbf{9}$ & $4.469 \mathrm{e}^{-04}$ & 19.79 & $<\mathbf{0 . 0 0 1}$ \\
\hline $\begin{array}{c}\text { Slow temperature increase vs. Rapid } \\
\text { temperature increase }\end{array}$ & $\mathbf{9}$ & $4.535 \mathrm{e}^{-04}$ & 20.09 & $<\mathbf{0 . 0 0 1}$ \\
\hline
\end{tabular}


ANOVA results comparing respiration rates of FlAp2 cultures exposed to control temperatures, a slow or rapid temperature increase.

\begin{tabular}{|c|c|c|c|c|c|}
\hline Variation & df & Sum of squares & Mean square & F & p-value \\
\hline Treatment & $\mathbf{2}$ & $6.520 \mathrm{e}^{-06}$ & $3.260 \mathrm{e}^{-06}$ & $\mathbf{6 . 2 2 6}$ & $\mathbf{0 . 0 2 0}$ \\
\hline Residuals & $\mathbf{9}$ & $4.710 \mathrm{e}^{-06}$ & $5.236 \mathrm{e}^{-07}$ & & \\
\hline Total & $\mathbf{1 1}$ & $1.120 \mathrm{e}^{-05}$ & & & \\
\hline
\end{tabular}

Tukeys post hoc comparing respiration rates of FlAp2 cultures exposed to control temperatures, a slow or rapid temperature increase

\begin{tabular}{|c|c|c|c|c|}
\hline Comparisons & df & Mean difference & $\mathbf{q}$ & p-value \\
\hline Control vs. Slow temperature increase & $\mathbf{9}$ & 0.001 & 2.995 & $\mathbf{0 . 1 4 1}$ \\
\hline Control vs. Rapid temperature increase & $\mathbf{9}$ & 0.002 & 4.955 & $\mathbf{0 . 0 1 7}$ \\
\hline $\begin{array}{c}\text { Slow temperature increase vs. Rapid } \\
\text { temperature increase }\end{array}$ & $\mathbf{9}$ & 0.001 & 1.960 & $\mathbf{0 . 3 8 8}$ \\
\hline
\end{tabular}

\section{Photosynthesis to respiration ratio}

ANOVA results comparing the photosynthesis to respiration ratio of NZ01 cultures exposed to control temperatures, a slow or rapid temperature increase

\begin{tabular}{|c|c|c|c|c|c|}
\hline Variation & df & Sum of squares & Mean square & F & p-value \\
\hline Treatment & $\mathbf{2}$ & 0.035 & 0.018 & $\mathbf{0 . 0 8 8}$ & $\mathbf{0 . 9 1 7}$ \\
\hline Residuals & $\mathbf{9}$ & 1.793 & 0.199 & & \\
\hline Total & $\mathbf{1 1}$ & 1.828 & & & \\
\hline
\end{tabular}

ANOVA results comparing the photosynthesis to respiration ratio of FlAp2 cultures exposed to control temperatures, a slow or rapid temperature increase.

\begin{tabular}{|c|c|c|c|c|c|}
\hline Variation & df & Sum of squares & Mean square & F & p-value \\
\hline Treatment & $\mathbf{2}$ & 6.844 & 3.422 & $\mathbf{1 9 6 . 0}$ & $<\mathbf{0 . 0 0 1}$ \\
\hline Residuals & $\mathbf{8}$ & 0.140 & 0.017 & & \\
\hline Total & $\mathbf{1 0}$ & 6.984 & & & \\
\hline
\end{tabular}


Tukeys post hoc comparing photosynthesis to respiration ratio of FlAp2 cultures exposed to control temperatures, a slow or rapid temperature increase

\begin{tabular}{|c|c|c|c|c|}
\hline Comparisons & df & Mean difference & $\mathbf{q}$ & p-value \\
\hline Control vs. Slow temperature increase & $\mathbf{8}$ & 1.741 & 26.35 & $<\mathbf{0 . 0 0 1}$ \\
\hline Control vs. Rapid temperature increase & $\mathbf{8}$ & 1.470 & 20.59 & $<\mathbf{0 . 0 0 1}$ \\
\hline $\begin{array}{c}\text { Slow temperature increase vs. Rapid } \\
\text { temperature increase }\end{array}$ & $\mathbf{8}$ & -0.271 & 3.798 & $\mathbf{0 . 0 6 4}$ \\
\hline
\end{tabular}

\section{Population growth}

ANOVA results comparing population growth rates of NZ01 cultures exposed to control temperatures, a slow or rapid temperature increase. Data was $\log _{10}$ transformed to meet ANOVA assumptions

\begin{tabular}{|c|c|c|c|c|c|}
\hline Variation & df & Sum of squares & Mean square & F & p-value \\
\hline Treatment & $\mathbf{2}$ & 2.784 & 1.392 & $\mathbf{5 6 . 5 7}$ & $<\mathbf{0 . 0 0 1}$ \\
\hline Residuals & $\mathbf{3 9}$ & 0.960 & 0.025 & & \\
\hline Total & $\mathbf{4 1}$ & 3.743 & & & \\
\hline
\end{tabular}

Tukeys post hoc comparing population growth rates of NZO1 cultures exposed to control temperatures, a slow or rapid temperature increase

\begin{tabular}{|c|c|c|c|c|}
\hline Comparisons & df & Mean difference & $\mathbf{q}$ & p-value \\
\hline Control vs. Slow temperature increase & $\mathbf{2}$ & 0.210 & 4.998 & $\mathbf{0 . 0 0 3}$ \\
\hline Control vs. Rapid temperature increase & $\mathbf{3 9}$ & 0.620 & 14.79 & $<\mathbf{0 . 0 0 1}$ \\
\hline $\begin{array}{c}\text { Slow temperature increase vs. Rapid } \\
\text { temperature increase }\end{array}$ & $\mathbf{4 1}$ & 0.410 & 9.788 & $<\mathbf{0 . 0 0 1}$ \\
\hline
\end{tabular}

ANOVA results comparing population growth rates of FlAp2 cultures exposed to control temperatures, a slow or rapid temperature increase

\begin{tabular}{|c|c|c|c|c|c|}
\hline Variation & df & Sum of squares & Mean square & F & p-value \\
\hline Treatment & $\mathbf{2}$ & 226.6 & 113.3 & $\mathbf{2 . 5}$ & $\mathbf{0 . 0 9 3}$ \\
\hline Residuals & $\mathbf{4 5}$ & 2040 & 45.33 & & \\
\hline Total & $\mathbf{4 7}$ & 2266 & & & \\
\hline
\end{tabular}




\section{Alkaline phosphatase assay}

ANOVA results comparing alkaline phosphatase activity of NZ01 cultures exposed to control temperatures, a slow or rapid temperature increase. Values were $\log _{10}$ transformed to meet ANOVA assumptions

\begin{tabular}{|c|c|c|c|c|c|}
\hline Variation & df & Sum of squares & Mean square & F & p-value \\
\hline Treatment & $\mathbf{2}$ & 0.221 & 0.110 & $\mathbf{7 . 0 6 7}$ & $\mathbf{0 . 0 2 7}$ \\
\hline Residuals & $\mathbf{6}$ & 0.094 & 0.016 & & \\
\hline Total & $\mathbf{8}$ & 0.315 & & & \\
\hline
\end{tabular}

Tukeys post hoc comparing alkaline phosphatase activity of NZ01 cultures exposed to control temperatures, a slow or rapid temperature increase

\begin{tabular}{|c|c|c|c|c|}
\hline Comparisons & df & Mean difference & $\mathbf{q}$ & p-value \\
\hline Control vs. Slow temperature increase & $\mathbf{6}$ & -0.381 & 5.270 & $\mathbf{0 . 0 2 3}$ \\
\hline Control vs. Rapid temperature increase & $\mathbf{6}$ & -0.233 & 3.229 & $\mathbf{0 . 1 3 5}$ \\
\hline $\begin{array}{c}\text { Slow temperature increase vs. Rapid } \\
\text { temperature increase }\end{array}$ & $\mathbf{6}$ & 0.147 & 2.043 & $\mathbf{0 . 3 7 9}$ \\
\hline
\end{tabular}

ANOVA results comparing alkaline phosphatase activity of FlAp2 cultures exposed to control temperatures, a slow or rapid temperature increase. Values were $\log _{10}$ transformed to meet ANOVA assumptions

\begin{tabular}{|c|c|c|c|c|c|}
\hline Variation & df & Sum of squares & Mean square & F & p-value \\
\hline Treatment & $\mathbf{2}$ & 0.164 & 0.082 & $\mathbf{5 . 8 3}$ & $\mathbf{0 . 0 3 9}$ \\
\hline Residuals & $\mathbf{6}$ & 0.085 & 0.014 & & \\
\hline Total & $\mathbf{8}$ & 0.249 & & & \\
\hline
\end{tabular}

Tukeys post hoc comparing alkaline phosphatase activity of FlAp2 cultures exposed to control temperatures, a slow or rapid temperature increase

\begin{tabular}{|c|c|c|c|c|}
\hline Comparisons & df & Mean difference & $\mathbf{q}$ & p-value \\
\hline Control vs. Slow temperature increase & $\mathbf{6}$ & -0.030 & 0.310 & $\mathbf{0 . 7 6 7}$ \\
\hline Control vs. Rapid temperature increase & $\mathbf{6}$ & 0.270 & 2.789 & $\mathbf{0 . 0 3 5}$ \\
\hline $\begin{array}{c}\text { Slow temperature increase vs. Rapid } \\
\text { temperature increase }\end{array}$ & $\mathbf{6}$ & 0.300 & 3.099 & $\mathbf{0 . 0 2 1}$ \\
\hline
\end{tabular}




\section{Appendix 2: Appendices for Chapter three}

\section{A2.1. Statistical tests for chapter three physiological data}

\section{Chlorophyll fluorescence statistical tests}

Multiple Unpaired two tailed T-test results comparing effective quantum yields of control cultures and those exposed to a slow temperature increase under an ambient nutrient regime.

\begin{tabular}{|c|c|c|c|}
\hline $\begin{array}{c}\text { Experimental } \\
\text { day }\end{array}$ & t-ratio & df & $\begin{array}{c}\text { p- } \\
\text { value }\end{array}$ \\
\hline 9 & 0.574 & 14 & $\mathbf{0 . 9 2 4}$ \\
\hline 10 & 1.678 & 14 & $\mathbf{0 . 5 7 6}$ \\
\hline 11 & 2.686 & 14 & $\mathbf{0 . 1 7 9}$ \\
\hline 12 & 0.051 & 14 & $\mathbf{0 . 9 9 3}$ \\
\hline 13 & 0.835 & 14 & $\mathbf{0 . 8 8 5}$ \\
\hline 14 & 0.107 & 14 & $\mathbf{0 . 9 9 3}$ \\
\hline 15 & 2.628 & 14 & $\mathbf{0 . 1 8 2}$ \\
\hline 16 & 1.240 & 14 & $\mathbf{0 . 7 3 9}$ \\
\hline 17 & 2.548 & 14 & $\mathbf{0 . 1 9 1}$ \\
\hline 18 & 1.508 & 14 & $\mathbf{0 . 6 3 3}$ \\
\hline 19 & 1.807 & 14 & $\mathbf{0 . 5 3 9}$ \\
\hline 20 & 3.207 & 14 & $\mathbf{0 . 0 7 3}$ \\
\hline 21 & 4.724 & 14 & $\mathbf{0 . 0 0 4}$ \\
\hline 22 & 5.579 & 14 & $\mathbf{0 . 0 0 1}$ \\
\hline & & & \\
\hline
\end{tabular}

Multiple Unpaired two tailed T-test results comparing maximum quantum yields of control cultures and those exposed to a slow temperature increase under an ambient nutrient regime.

\begin{tabular}{|c|c|c|c|}
\hline $\begin{array}{c}\text { Experimental } \\
\text { day }\end{array}$ & t-ratio & df & p-value \\
\hline 9 & 2.786 & 14 & $\mathbf{0 . 0 9 8}$ \\
\hline 10 & 5.434 & 14 & $\mathbf{0 . 0 0 1}$ \\
\hline 11 & 2.775 & 14 & $\mathbf{0 . 0 9 8}$ \\
\hline 12 & 1.406 & 14 & $\mathbf{0 . 4 9 7}$ \\
\hline
\end{tabular}




\begin{tabular}{|c|c|c|c|}
\hline 13 & 0.648 & 14 & $\mathbf{0 . 7 7 6}$ \\
\hline 14 & 0.575 & 14 & $\mathbf{0 . 7 7 6}$ \\
\hline 15 & 1.533 & 14 & $\mathbf{0 . 4 9 7}$ \\
\hline 16 & 1.615 & 14 & $\mathbf{0 . 4 9 7}$ \\
\hline 17 & 5.412 & 14 & $\mathbf{0 . 0 0 1}$ \\
\hline 18 & 7.214 & 14 & $<\mathbf{0 . 0 0 1}$ \\
\hline 19 & 8.44 & 14 & $<\mathbf{0 . 0 0 1}$ \\
\hline 20 & 6.275 & 14 & $<\mathbf{0 . 0 0 1}$ \\
\hline 21 & 11.30 & 14 & $<\mathbf{0 . 0 0 1}$ \\
\hline 22 & 9.747 & 14 & $<\mathbf{0 . 0 0 1}$ \\
\hline
\end{tabular}

Multiple Unpaired two tailed T-test results comparing effective quantum yields of control cultures and those exposed to a slow temperature increase under an imbalanced nutrient regime.

\begin{tabular}{|c|c|c|c|}
\hline $\begin{array}{c}\text { Experimental } \\
\text { day }\end{array}$ & t-ratio & df & p-value \\
\hline 9 & 3.673 & 14 & 0.025 \\
\hline 10 & 3.207 & 14 & 0.050 \\
\hline 11 & 4.398 & 14 & 0.007 \\
\hline 12 & 3.154 & 14 & 0.050 \\
\hline 13 & 2.963 & 14 & 0.060 \\
\hline 14 & 0.881 & 14 & 0.632 \\
\hline 15 & 3.281 & 14 & 0.048 \\
\hline 16 & 0.487 & 14 & 0.634 \\
\hline 17 & 2.050 & 14 & 0.218 \\
\hline 18 & 1.649 & 14 & 0.322 \\
\hline 19 & 2.747 & 14 & 0.076 \\
\hline 20 & 5.194 & 14 & 0.002 \\
\hline 21 & 18.94 & 14 & $<0.001$ \\
\hline 22 & 10.90 & 14 & $<0.001$ \\
\hline
\end{tabular}


Results of a repeated measures ANOVA comparing how the effective quantum yields of control cultures and those exposed to a slow temperature increase change over time, under an imbalanced nutrient regime.

\begin{tabular}{|c|c|c|c|c|c|}
\hline Variation & df & Sum of squares & Mean square & F & p-value \\
\hline Time & $\mathbf{8}$ & 0.074 & 0.009 & $\mathbf{1 2 . 6 2}$ & $<\mathbf{0 . 0 0 1}$ \\
\hline Treatment & $\mathbf{1}$ & 0.440 & 0.440 & $\mathbf{3 3 0 . 4}$ & $<\mathbf{0 . 0 0 1}$ \\
\hline Interaction & $\mathbf{8}$ & 0.092 & 0.011 & $\mathbf{1 6 . 8 5}$ & $<\mathbf{0 . 0 0 1}$ \\
\hline Residual & $\mathbf{5 6}$ & 0.038 & 0.001 & & \\
\hline Total & $\mathbf{7 3}$ & & & & \\
\hline
\end{tabular}

Dunnet's multiple comparison test comparing the maximum quantum yield of cultures averaged over the first seven days of the active experimental period (when all cultures including the treatments were at $25^{\circ} \mathrm{C}$ ), with the yields on days 16-22. Due to the interaction of the repeated measures ANOVA as seen in the table above these comparisons were performed separately for the control and treatment cultures.

\begin{tabular}{|c|c|c|c|}
\hline Comparison & $\begin{array}{c}\text { Mean } \\
\text { difference }\end{array}$ & $\begin{array}{c}\mathbf{9 5 \%} \text { confidence } \\
\text { interval of difference }\end{array}$ & Adjusted p-value \\
\hline Control cultures & & & \\
\hline Average vs. day 16 & -0.039 & -0.075 to -0.004 & $\mathbf{0 . 0 2 5}$ \\
\hline Average vs. day 17 & -0.013 & -0.049 to 0.022 & $\mathbf{0 . 8 6 5}$ \\
\hline Average vs. day 18 & 0.046 & 0.010 to 0.082 & $\mathbf{0 . 0 0 6}$ \\
\hline Average vs. day 19 & -0.048 & -0.084 t o -0.013 & $\mathbf{0 . 0 0 4}$ \\
\hline Average vs. day 20 & -0.018 & -0.054 to 0.018 & $\mathbf{0 . 6 3 7}$ \\
\hline Average vs. day 21 & -0.031 & -0.067 to 0.005 & $\mathbf{0 . 1 1 4}$ \\
\hline Average vs. day 22 & -0.032 & -0.067 to 0.004 & $\mathbf{0 . 1 0 5}$ \\
\hline Treatment cultures & & & $\mathbf{0 . 2 2 8}$ \\
\hline Average vs. day 16 & 0.027 & -0.009 to 0.062 & $\mathbf{0 . 2 2 8}$ \\
\hline Average vs. day 17 & 0.027 & -0.009 to 0.063 & $<\mathbf{0 . 0 0 1}$ \\
\hline Average vs. day 18 & 0.080 & 0.040 to 0.111 & $<\mathbf{0 . 0 0 1}$ \\
\hline Average vs. day 19 & 0.063 & 0.028 to 0.099 & $<\mathbf{0 . 0 0 1}$ \\
\hline Average vs. day 20 & 0.086 & 0.050 to 0.121 & $<\mathbf{0 . 0 0 1}$ \\
\hline Average vs. day 21 & 0.083 & 0.048 to 0.119 & $\mathbf{0 . 0 0 4}$ \\
\hline Average vs. day 22 & 0.048 & 0.012 to 0.083 & \\
\hline
\end{tabular}


Multiple Unpaired two tailed T-test results comparing effective quantum yields of control cultures and those exposed to a slow temperature increase under an enriched nutrient regime.

\begin{tabular}{|c|c|c|c|}
\hline $\begin{array}{c}\text { Experimental } \\
\text { day }\end{array}$ & t-ratio & df & p-value \\
\hline 9 & 0.761 & 14 & $\mathbf{0 . 8 4 2}$ \\
\hline 10 & 5.849 & 14 & $<\mathbf{0 . 0 0 1}$ \\
\hline 11 & 6.457 & 14 & $<\mathbf{0 . 0 0 1}$ \\
\hline 12 & 3.828 & 14 & $\mathbf{0 . 0 1 5}$ \\
\hline 13 & 1.941 & 14 & $\mathbf{0 . 3 5 5}$ \\
\hline 14 & 0.309 & 14 & $\mathbf{0 . 8 4 2}$ \\
\hline 15 & 4.066 & 14 & $\mathbf{0 . 0 1 0}$ \\
\hline 16 & 5.297 & 14 & $\mathbf{0 . 0 0 1}$ \\
\hline 17 & 0.673 & 14 & $\mathbf{0 . 8 4 2}$ \\
\hline 18 & 1.747 & 14 & $\mathbf{0 . 3 5 5}$ \\
\hline 19 & 1.957 & 14 & $\mathbf{0 . 3 5 5}$ \\
\hline 20 & 5.786 & 14 & $<\mathbf{0 . 0 0 1}$ \\
\hline 21 & 8.304 & 14 & $<\mathbf{0 . 0 0 1}$ \\
\hline 22 & 2.971 & 14 & $\mathbf{0 . 0 6 9}$ \\
\hline
\end{tabular}

Multiple Unpaired two tailed T-test results comparing maximum quantum yields of control cultures and those exposed to a slow temperature increase under an enriched nutrient regime.

\begin{tabular}{|c|c|c|c|}
\hline $\begin{array}{c}\text { Experimental } \\
\text { day }\end{array}$ & t-ratio & df & p-value \\
\hline 9 & 1.443 & 14 & $\mathbf{0 . 6 0 9}$ \\
\hline 10 & 0.202 & 14 & $\mathbf{0 . 9 7 5}$ \\
\hline 11 & 2.313 & 14 & $\mathbf{0 . 2 2 9}$ \\
\hline 12 & 0.117 & 14 & $\mathbf{0 . 9 7 5}$ \\
\hline 13 & 0.415 & 14 & $\mathbf{0 . 9 6 9}$ \\
\hline 14 & 1.429 & 14 & $\mathbf{0 . 6 0 9}$ \\
\hline 15 & 2.909 & 14 & $\mathbf{0 . 0 9 8}$ \\
\hline 16 & 2.784 & 14 & $\mathbf{0 . 1 1 1}$ \\
\hline 17 & 1.551 & 14 & $\mathbf{0 . 6 0 5}$ \\
\hline 18 & 4.332 & 14 & $\mathbf{0 . 0 0 7}$ \\
\hline 19 & 6.285 & 14 & $<\mathbf{0 . 0 0 1}$ \\
\hline
\end{tabular}




\begin{tabular}{|l|l|l|l|}
\hline 20 & 8.077 & 14 & $<\mathbf{0 . 0 0 1}$ \\
\hline 21 & 9.792 & 14 & $<\mathbf{0 . 0 0 1}$ \\
\hline 22 & 8.596 & 14 & $<\mathbf{0 . 0 0 1}$ \\
\hline
\end{tabular}

\section{Respiratory and photosynthetic Oxygen flux}

\section{Gross photosynthesis}

Two way- ANOVA results comparing gross photosynthesis rates of cultures exposed to control temperatures or a slow temperature increase, under an ambient, imbalanced or enriched nutrient regime

\begin{tabular}{|c|c|c|c|c|c|}
\hline Variation & df & Sum of squares & Mean square & F & p-value \\
\hline Interaction & $\mathbf{2}$ & $2.003 \mathrm{e}^{-6}$ & $1.002 \mathrm{e}^{-6}$ & $\mathbf{6 . 6 3 8}$ & $\mathbf{0 . 0 0 7}$ \\
\hline $\begin{array}{c}\text { Nutrient } \\
\text { level }\end{array}$ & $\mathbf{2}$ & $2.639 \mathrm{e}^{-6}$ & $1.319 \mathrm{e}^{-6}$ & $\mathbf{8 . 7 4 4}$ & $\mathbf{0 . 0 0 2}$ \\
\hline $\begin{array}{c}\text { Temperature } \\
\text { level }\end{array}$ & $\mathbf{1}$ & $4.583 \mathrm{e}^{-07}$ & $4.583 \mathrm{e}^{-07}$ & $\mathbf{3 . 0 3 7}$ & $\mathbf{0 . 0 9 9}$ \\
\hline Residual & $\mathbf{1 7}$ & $2.565 \mathrm{e}^{-6}$ & $1.509 \mathrm{e}^{-07}$ & & \\
\hline Total & $\mathbf{2 1}$ & & & & \\
\hline
\end{tabular}

Tukeys multiple comparisons test comparing gross photosynthesis rates of cultures under an ambient, imbalanced or enriched nutrient regime at either control or treatment temperatures

\begin{tabular}{|c|c|c|c|}
\hline Comparisons & Mean difference & $\begin{array}{c}\mathbf{9 5 . 0 0 \%} \text { Confidence } \\
\text { interval of difference }\end{array}$ & p-value \\
\hline $\mathbf{2 5}^{\circ} \boldsymbol{C}$ & & & \\
\hline Ambient vs. Imbalanced & $2.956 \mathrm{e}^{-5}$ & $-5.373 \mathrm{e}^{-4}$ to $9.849 \mathrm{e}^{-4}$ & $\mathbf{0 . 7 3 5}$ \\
\hline Ambient vs. Enriched & -0.001 & $-6.574 \mathrm{e}^{-4}$ to $8.648 \mathrm{e}^{-4}$ & $\mathbf{0 . 9 3 5}$ \\
\hline Enriched vs. Imbalanced & -0.001 & $-8.247 \mathrm{e}^{-4}$ to $5.845 \mathrm{e}^{-4}$ & $\mathbf{0 . 9 0 1}$ \\
\hline $\mathbf{3 4}^{\circ} \boldsymbol{C}$ & & & $\mathbf{0 . 0 1 3}$ \\
\hline Ambient vs. Imbalanced & 0.001 & $1.801 \mathrm{e}^{-4}$ to $1.589 \mathrm{e}^{-4}$ & $<\mathbf{0 . 0 0 1}$ \\
\hline Ambient vs. Enriched & 0.002 & $-2.043 \mathrm{e}^{-4}$ to $2.274 \mathrm{e}^{-4}$ to $1.389 \mathrm{e}^{-4}$ & $\mathbf{0 . 0 5 8}$ \\
\hline Enriched vs. Imbalanced & 0.001 & & \\
\hline
\end{tabular}


Tukeys multiple comparisons test comparing gross photosynthesis rates of cultures under an ambient, imbalanced or enriched nutrient regime, regardless of temperature

\begin{tabular}{|c|c|c|c|}
\hline Comparisons & Mean difference & $\begin{array}{c}\text { 95.00\% Confidence interval } \\
\text { of difference }\end{array}$ & p-value \\
\hline Ambient vs. Imbalanced & $6.252 \mathrm{e}^{-4}$ & $1.094 \mathrm{e}^{-4}$ to $1.141 \mathrm{e}^{-3}$ & $\mathbf{0 . 0 1 7}$ \\
\hline Ambient vs. Enriched & $9.073 \mathrm{e}^{-4}$ & $3.915 \mathrm{e}^{-4}$ to $1.423 \mathrm{e}^{-3}$ & $\mathbf{0 . 0 0 1}$ \\
\hline Enriched vs. Imbalanced & $2.821 \mathrm{e}^{-4}$ & $2.162 \mathrm{e}^{-4}$ to $7.803 \mathrm{e}^{-4}$ & $\mathbf{0 . 3 3 8}$ \\
\hline
\end{tabular}

Unpaired two tailed T-test results determining the effect of temperature on gross photosynthetic rates within each nutrient regime separately

\begin{tabular}{|c|c|c|c|}
\hline Treatment & df & t-ratio & p-value \\
\hline Ambient, $25^{\circ} \mathrm{C} v s .34^{\circ} \mathrm{C}$ & $\mathbf{5}$ & 2.656 & $\mathbf{0 . 0 4 5}$ \\
\hline $\begin{array}{c}\text { Imbalanced, } 25^{\circ} \mathrm{C} v s . \\
34^{\circ} \mathrm{C}\end{array}$ & $\mathbf{6}$ & 1.824 & $\mathbf{0 . 1 1 8}$ \\
\hline Enriched, $25^{\circ} \mathrm{C} v \mathrm{~s} .34^{\circ} \mathrm{C}$ & $\mathbf{6}$ & 1.662 & $\mathbf{0 . 1 4 8}$ \\
\hline
\end{tabular}

\section{Respiration}

Two way- ANOVA results comparing respiration rates of cultures exposed to control temperatures or a slow temperature increase, under an ambient, imbalanced or enriched nutrient regime

\begin{tabular}{|c|c|c|c|c|c|}
\hline Variation & df & Sum of squares & Mean square & F & p-value \\
\hline Interaction & $\mathbf{2}$ & $4.864 \mathrm{e}^{-6}$ & $2.432 \mathrm{e}^{-6}$ & $\mathbf{1 0 . 7 1}$ & $\mathbf{0 . 0 0 1}$ \\
\hline Nutrient level & $\mathbf{2}$ & $3.904 \mathrm{e}^{-6}$ & $1.952 \mathrm{e}^{-6}$ & $\mathbf{8 . 5 9 5}$ & $\mathbf{0 . 0 0 3}$ \\
\hline $\begin{array}{c}\text { Temperature } \\
\text { level }\end{array}$ & $\mathbf{1}$ & $1.532 \mathrm{e}^{-5}$ & $1.532 \mathrm{e}^{-6}$ & $\mathbf{6 7 . 4 5}$ & $<\mathbf{0 . 0 0 1}$ \\
\hline Residual & $\mathbf{1 7}$ & $3.861 \mathrm{e}^{-6}$ & $2.271 \mathrm{e}^{-7}$ & & \\
\hline Total & $\mathbf{2 1}$ & & & & \\
\hline
\end{tabular}

Tukeys multiple comparisons test comparing respiration rates of cultures under an ambient, imbalanced or enriched nutrient regime at either control or treatment temperatures

\begin{tabular}{|c|c|c|c|}
\hline Comparisons & $\begin{array}{c}\text { Mean } \\
\text { difference }\end{array}$ & $\begin{array}{c}\mathbf{9 5 . 0 0 \%} \text { Confidence } \\
\text { interval of } \\
\text { difference }\end{array}$ & p-value \\
\hline $\mathbf{2 5}^{\circ} \mathbf{C}$ & & & \\
\hline Ambient vs. Imbalanced & $-2.037 \mathrm{e}^{-4}$ & $-1.137 \mathrm{e}^{-3}$ to $7.300 \mathrm{e}^{-4}$ & $\mathbf{0 . 8 4 3}$ \\
\hline Ambient vs. Enriched & $1.448 \mathrm{e}^{-4}$ & $-7.889 \mathrm{e}^{-4}$ to $1.079 \mathrm{e}^{-3}$ & $\mathbf{0 . 9 1 7}$ \\
\hline Enriched vs. Imbalanced & $3.485 \mathrm{e}^{-4}$ & $-5.160 \mathrm{e}^{-4}$ to $1.213 \mathrm{e}^{-3}$ & $\mathbf{0 . 5 6 6}$ \\
\hline $\mathbf{3 4}^{\circ} \mathbf{C}$ & & & \\
\hline Ambient vs. Imbalanced & $-1.281 \mathrm{e}^{-4}$ & $-2.145 \mathrm{e}^{-3}$ to $-4.162 \mathrm{e}^{-4}$ & $\mathbf{0 . 0 0 4}$ \\
\hline Ambient vs. Enriched & $-2.143 \mathrm{e}^{-4}$ & $-3.008 \mathrm{e}^{-3}$ to $-1.279 \mathrm{e}^{-3}$ & $<\mathbf{0 . 0 0 1}$ \\
\hline Enriched vs. Imbalanced & $-8.627 \mathrm{e}^{-4}$ & $-1.727 \mathrm{e}^{-3}$ to $1.737 \mathrm{e}^{-6}$ & $\mathbf{0 . 0 5 1}$ \\
\hline
\end{tabular}


Tukeys multiple comparisons test comparing respiration rates of cultures under an ambient, imbalanced or enriched nutrient regime, regardless of temperature

\begin{tabular}{|c|c|c|c|}
\hline Comparisons & Mean difference & $\begin{array}{c}\mathbf{9 5 . 0 0 \%} \text { Confidence } \\
\text { interval of difference }\end{array}$ & p-value \\
\hline Ambient vs. Imbalanced & $-9.396 \mathrm{e}^{-4}$ & $-1.572 \mathrm{e}^{-3}$ to $-3.069 \mathrm{e}^{-4}$ & $\mathbf{0 . 0 0 3 8}$ \\
\hline Ambient vs. Enriched & $-1.197 \mathrm{e}^{-3}$ & $-1.829 \mathrm{e}^{-3}$ to $-05.640 \mathrm{e}^{-4}$ & $\mathbf{0 . 0 0 0 4}$ \\
\hline Enriched vs. Imbalanced & $-2.571 \mathrm{e}^{-4}$ & $-8.684 \mathrm{e}^{-4}$ to $3.541 \mathrm{e}^{-4}$ & $\mathbf{0 . 5 3 9 4}$ \\
\hline
\end{tabular}

Unpaired two tailed T-test results determining the effect of temperature on respiration rates within each nutrient regime separately

\begin{tabular}{|c|c|c|c|}
\hline Treatment & df & t-ratio & p-value \\
\hline Ambient, $25^{\circ} \mathrm{C}$ vs. $34^{\circ} \mathrm{C}$ & $\mathbf{5}$ & 5.862 & $\mathbf{0 . 0 0 2}$ \\
\hline Imbalanced, $25^{\circ} \mathrm{C}$ vs. $34^{\circ} \mathrm{C}$ & $\mathbf{6}$ & 5.843 & $\mathbf{0 . 0 0 1}$ \\
\hline Enriched, $25^{\circ} \mathrm{C}$ vs. $34^{\circ} \mathrm{C}$ & $\mathbf{6}$ & 1.683 & $\mathbf{0 . 1 4 3}$ \\
\hline
\end{tabular}

\section{Photosynthesis: respiration ratio}

Two way- ANOVA results comparing the P:R ratio of cultures exposed to control temperatures or a slow temperature increase, under an ambient, imbalanced or enriched nutrient regime

\begin{tabular}{|c|c|c|c|c|c|}
\hline Variation & df & Sum of squares & Mean square & F & p-value \\
\hline Interaction & $\mathbf{2}$ & 0.170 & 0.085 & $\mathbf{5 . 6 3 0}$ & $\mathbf{0 . 0 1 3}$ \\
\hline $\begin{array}{c}\text { Nutrient } \\
\text { level }\end{array}$ & $\mathbf{2}$ & 0.280 & 0.140 & $\mathbf{9 . 2 8 2}$ & $\mathbf{0 . 0 0 2}$ \\
\hline $\begin{array}{c}\text { Temperature } \\
\text { level }\end{array}$ & $\mathbf{1}$ & 4.787 & 4.787 & $\mathbf{3 1 7 . 1}$ & $<\mathbf{0 . 0 0 0 1}$ \\
\hline Residual & $\mathbf{1 7}$ & 0.257 & 0.015 & & \\
\hline Total & $\mathbf{2 1}$ & & & & \\
\hline
\end{tabular}

Tukeys multiple comparisons test comparing the P:R ratio of cultures under an ambient, imbalanced or enriched nutrient regime at either control or treatment temperatures

\begin{tabular}{|c|c|c|c|}
\hline Comparisons & $\begin{array}{c}\text { Mean } \\
\text { difference }\end{array}$ & $\begin{array}{c}\mathbf{9 5 . 0 0 \%} \text { Confidence interval of } \\
\text { difference }\end{array}$ & p-value \\
\hline $\mathbf{2 5}^{\circ} \mathbf{C}$ & & & \\
\hline Ambient vs. Imbalanced & -0.156 & -0.397 to 0.085 & $\mathbf{0 . 2 4 8}$ \\
\hline Ambient vs. Enriched & 0.304 & 0.063 to 0.545 & $\mathbf{0 . 0 1 3}$ \\
\hline Enriched vs. Imbalanced & 0.460 & 0.237 to 0.683 & $<\mathbf{0 . 0 0 1}$ \\
\hline $\mathbf{3 4}^{\circ} \mathbf{C}$ & & & $\mathbf{0 . 8 9 2}$ \\
\hline Ambient vs. Imbalanced & 0.040 & -0.183 to 0.263 & $\mathbf{0 . 5 8 3}$ \\
\hline Ambient vs. Enriched & 0.087 & -0.136 to 0.310 & $\mathbf{0 . 8 4 8}$ \\
\hline Enriched vs. Imbalanced & 0.048 & -0.175 to 0.271 & \\
\hline
\end{tabular}


Tukeys multiple comparisons test comparing the P:R ratio of cultures under an ambient, imbalanced or enriched nutrient regime, regardless of temperature

\begin{tabular}{|c|c|c|c|}
\hline Comparisons & $\begin{array}{c}\text { Mean } \\
\text { difference }\end{array}$ & $\begin{array}{c}\mathbf{9 5 . 0 0 \%} \text { Confidence interval of } \\
\text { difference }\end{array}$ & p-value \\
\hline Ambient vs. Imbalanced & -0.124 & -0.287 to 0.039 & $\mathbf{0 . 1 5 4}$ \\
\hline Ambient vs. Enriched & 0.130 & -0.034 to 0.293 & $\mathbf{0 . 1 3 4}$ \\
\hline Enriched vs. Imbalanced & 0.254 & 0.096 to 0.411 & $\mathbf{0 . 0 0 2}$ \\
\hline
\end{tabular}

Unpaired two tailed T-test results determining the effect of temperature on the P:R ratio within each nutrient regime separately, the test for the imbalanced nutrient regime is Welchs corrected due to unequal standard deviations between temperature treatments

\begin{tabular}{|c|c|c|c|}
\hline Treatment & $d f$ & t-ratio & $p$-value \\
\hline Ambient, $25^{\circ} \mathrm{C}$ vs. $34^{\circ} \mathrm{C}$ & $\mathbf{5}$ & 10.94 & $<\mathbf{0 . 0 0 1}$ \\
\hline $\begin{array}{c}\text { Imbalanced, } 25^{\circ} \mathrm{C} \text { vs. } \\
34^{\circ} \mathrm{C}\end{array}$ & $\mathbf{3 . 3 4 4}$ & 11.39 & $\mathbf{0 . 0 4 1}$ \\
\hline $\begin{array}{c}\text { Enriched, } 25^{\circ} \mathrm{C} \text { vs. } \\
34^{\circ} \mathrm{C}\end{array}$ & $\mathbf{6}$ & 8.709 & $<\mathbf{0 . 0 0 1}$ \\
\hline
\end{tabular}

\section{Chlorophyll a content}

Two way-ANOVA results comparing the chlorophyll a content of cultures exposed to control temperatures or a slow temperature increase, under an ambient, imbalanced or enriched nutrient regime

\begin{tabular}{|c|c|c|c|c|c|}
\hline Variation & df & Sum of squares & Mean square & F & p-value \\
\hline Interaction & $\mathbf{2}$ & $1.646 \mathrm{e}^{-4}$ & $8.232 \mathrm{e}^{-5}$ & $\mathbf{1 . 1 1 4}$ & $\mathbf{0 . 3 5 0}$ \\
\hline $\begin{array}{c}\text { Nutrient } \\
\text { level }\end{array}$ & $\mathbf{2}$ & $4.687 \mathrm{e}^{-4}$ & $2.344 \mathrm{e}^{-4}$ & $\mathbf{3 . 1 7 0}$ & $\mathbf{0 . 0 6 6}$ \\
\hline $\begin{array}{c}\text { Temperature } \\
\text { level }\end{array}$ & $\mathbf{1}$ & $1.647 \mathrm{e}^{-4}$ & $1.647 \mathrm{e}^{-4}$ & $\mathbf{2 . 2 2 8}$ & $\mathbf{0 . 1 5 3}$ \\
\hline Residual & $\mathbf{1 8}$ & $1.331 \mathrm{e}^{-3}$ & $7.392 \mathrm{e}^{-5}$ & & \\
\hline Total & $\mathbf{2 2}$ & & & & \\
\hline
\end{tabular}

Unpaired two tailed T-test results determining the effect of temperature on chlorophyll a content within each nutrient regime separately

\begin{tabular}{|c|c|c|c|}
\hline Treatment & df & t-ratio & p-value \\
\hline Ambient, $25^{\circ} \mathrm{C}$ vs. $34^{\circ} \mathrm{C}$ & $\mathbf{6}$ & 1.695 & $\mathbf{0 . 1 4 1}$ \\
\hline Imbalanced, $25^{\circ} \mathrm{C}$ vs. $34^{\circ} \mathrm{C}$ & $\mathbf{6}$ & 1.584 & $\mathbf{0 . 1 6 4}$ \\
\hline Enriched, $25^{\circ} \mathrm{C}$ vs. $34^{\circ} \mathrm{C}$ & $\mathbf{6}$ & 0.533 & $\mathbf{0 . 7 9 3}$ \\
\hline
\end{tabular}




\section{Carotenoid content}

Two way- ANOVA results comparing the carotenoid content of cultures exposed to control temperatures or a slow temperature increase, under an ambient, imbalanced or enriched nutrient regime

\begin{tabular}{|c|c|c|c|c|c|}
\hline Variation & df & Sum of squares & Mean square & F & p-value \\
\hline Interaction & $\mathbf{2}$ & $7.829 \mathrm{e}^{-5}$ & $3.915 \mathrm{e}^{-5}$ & $\mathbf{0 . 3 4 6}$ & $\mathbf{0 . 7 1 2}$ \\
\hline $\begin{array}{c}\text { Nutrient } \\
\text { level }\end{array}$ & $\mathbf{2}$ & $1.996 \mathrm{e}^{-5}$ & $9.978 \mathrm{e}^{-6}$ & $\mathbf{0 . 0 8 8}$ & $\mathbf{0 . 9 1 6}$ \\
\hline $\begin{array}{c}\text { Temperature } \\
\text { level }\end{array}$ & $\mathbf{1}$ & $2.491 \mathrm{e}^{-4}$ & $2.491 \mathrm{e}^{-4}$ & $\mathbf{2 . 2 0 5}$ & $\mathbf{0 . 1 5 5}$ \\
\hline Residual & $\mathbf{1 8}$ & $2.034 \mathrm{e}^{-3}$ & $1.130 \mathrm{e}^{-4}$ & & \\
\hline Total & $\mathbf{2 2}$ & & & & \\
\hline
\end{tabular}

Unpaired two tailed T-test results determining the effect of temperature on carotenoid content within each nutrient regime separately

\begin{tabular}{|c|c|c|c|}
\hline Treatment & df & t-ratio & p-value \\
\hline Ambient, $25^{\circ} \mathrm{C}$ vs. $34^{\circ} \mathrm{C}$ & $\mathbf{6}$ & 1.675 & $\mathbf{0 . 1 4 5}$ \\
\hline $\begin{array}{c}\text { Imbalanced, } 25^{\circ} \mathrm{C} \text { vs. } \\
34^{\circ} \mathrm{C}\end{array}$ & $\mathbf{6}$ & 1.368 & $\mathbf{0 . 2 2 0}$ \\
\hline $\begin{array}{c}\text { Enriched, } 25^{\circ} \mathrm{C} \text { vs. } \\
34^{\circ} \mathrm{C}\end{array}$ & $\mathbf{6}$ & 0.146 & $\mathbf{0 . 8 8 9}$ \\
\hline
\end{tabular}

\section{The ratio of carotenoids to chlorophyll a}

Two way- ANOVA results comparing the ratio of carotenoids to chlorophyll a of cultures exposed to control temperatures or a slow temperature increase, under an ambient, imbalanced or enriched nutrient regime

\begin{tabular}{|c|c|c|c|c|c|}
\hline Variation & df & Sum of squares & Mean square & F & p-value \\
\hline Interaction & $\mathbf{2}$ & 0.023 & 0.011 & $\mathbf{1 . 7 5 0}$ & $\mathbf{0 . 2 0 2}$ \\
\hline $\begin{array}{c}\text { Nutrient } \\
\text { level }\end{array}$ & $\mathbf{2}$ & 0.314 & 0.157 & $\mathbf{2 4 . 0 8}$ & $<\mathbf{0 . 0 0 1}$ \\
\hline $\begin{array}{c}\text { Temperature } \\
\text { level }\end{array}$ & $\mathbf{1}$ & 0.0061 & 0.006 & $\mathbf{0 . 9 3 7}$ & $\mathbf{0 . 3 4 6}$ \\
\hline Residual & $\mathbf{1 8}$ & 0.117 & 0.007 & & \\
\hline Total & $\mathbf{2 2}$ & & & & \\
\hline
\end{tabular}


Tukeys multiple comparisons test comparing the ratio of carotenoids to chlorophyll a of cultures under an ambient, imbalanced or enriched nutrient regime, regardless of temperature

\begin{tabular}{|c|c|c|c|}
\hline Comparisons & Mean difference & $\begin{array}{c}\text { 95.00\% } \\
\text { Confidence } \\
\text { interval of } \\
\text { difference }\end{array}$ & p-value \\
\hline Ambient vs. Imbalanced & -0.124 & -0.287 to 0.039 & $\mathbf{0 . 1 5 4}$ \\
\hline Ambient vs. Enriched & 0.130 & -0.034 to 0.293 & $\mathbf{0 . 1 3 4}$ \\
\hline Enriched vs. Imbalanced & 0.254 & 0.096 to 0.411 & $\mathbf{0 . 0 0 2}$ \\
\hline
\end{tabular}

Unpaired two tailed T-test results determining the effect of temperature on the ratio of carotenoids to chlorophyll a within each nutrient regime separately

\begin{tabular}{|c|c|c|c|}
\hline Treatment & df & t-ratio & p-value \\
\hline Ambient, $25^{\circ} \mathrm{C}$ vs. $34^{\circ} \mathrm{C}$ & $\mathbf{6}$ & 0.235 & $\mathbf{0 . 8 2 2}$ \\
\hline $\begin{array}{c}\text { Imbalanced, } 25^{\circ} \mathrm{C} \text { vs. } \\
34^{\circ} \mathrm{C}\end{array}$ & $\mathbf{6}$ & 0.357 & $\mathbf{0 . 7 3 3}$ \\
\hline $\begin{array}{c}\text { Enriched, } 25^{\circ} \mathrm{C} \text { vs. } \\
34^{\circ} \mathrm{C}\end{array}$ & $\mathbf{6}$ & 1.456 & $\mathbf{0 . 0 8 9}$ \\
\hline
\end{tabular}

\section{Alkaline phosphatase activity}

Two way-ANOVA results comparing the alkaline phosphatase activity of cultures exposed to control temperatures or a slow temperature increase, under an ambient, imbalanced or enriched nutrient regime

\begin{tabular}{|c|c|c|c|c|c|}
\hline Variation & df & Sum of squares & Mean square & F & p-value \\
\hline Interaction & $\mathbf{2}$ & 0.701 & 0.3505 & $\mathbf{6 . 9 5 0}$ & $\mathbf{0 . 0 0 6}$ \\
\hline $\begin{array}{c}\text { Nutrient } \\
\text { level }\end{array}$ & $\mathbf{2}$ & 4.673 & 2.336 & $\mathbf{4 6 . 3 3}$ & $<\mathbf{0 . 0 0 1}$ \\
\hline $\begin{array}{c}\text { Temperature } \\
\text { level }\end{array}$ & $\mathbf{1}$ & 1.550 & 1.550 & $\mathbf{3 0 . 7 3}$ & $<\mathbf{0 . 0 0 1}$ \\
\hline Residual & $\mathbf{1 8}$ & 0.9078 & 0.050 & & \\
\hline Total & $\mathbf{2 2}$ & & & & \\
\hline
\end{tabular}

Tukeys multiple comparisons test comparing the alkaling phosphatase activity under an ambient, imbalanced or enriched nutrient regime, regardless of temperature

\begin{tabular}{|c|c|c|c|}
\hline Comparisons & Mean difference & $\begin{array}{c}\mathbf{9 5 . 0 0 \%} \\
\text { Confidence } \\
\text { interval of } \\
\text { difference }\end{array}$ & p-value \\
\hline Ambient vs. Imbalanced & -0.888 & -1.175 to 0.601 & $<\mathbf{0 . 0 0 1}$ \\
\hline Ambient vs. Enriched & 0.090 & -0.197 to 0.3763 & $\mathbf{0 . 7 0 8}$ \\
\hline Enriched vs. Imbalanced & 0.978 & 0.691 to 1.264 & $<\mathbf{0 . 0 0 1}$ \\
\hline
\end{tabular}


Unpaired two tailed T-test results determining the effect of temperature on alkaline phosphatase activity within the ambient and enriched regimes separately.

\begin{tabular}{|c|c|c|c|}
\hline Treatment & df & t-ratio & p-value \\
\hline Ambient, $25^{\circ} \mathrm{C} v s .34^{\circ} \mathrm{C}$ & $\mathbf{6}$ & 5.373 & $\mathbf{0 . 0 0 2}$ \\
\hline $\begin{array}{c}\text { Enriched, } 25^{\circ} \mathrm{C} v s . \\
34^{\circ} \mathrm{C}\end{array}$ & $\mathbf{6}$ & 1.274 & $\mathbf{0 . 2 5 0}$ \\
\hline
\end{tabular}

Two tailed Mann Whitney U-test determining the effect of temperature on alkaline phosphatase activity for cultures under the imbalanced nutrient regime

\begin{tabular}{|c|c|c|c|}
\hline Treatment & Sum of ranks & $\begin{array}{c}\text { Difference between } \\
\text { medians }\end{array}$ & p-value \\
\hline $\begin{array}{c}\text { Imbalanced, } 25^{\circ} \mathrm{C} v s . \\
34^{\circ} \mathrm{C}\end{array}$ & 10,26 & 1.195 & $\mathbf{0 . 0 2 9}$ \\
& & & \\
\hline
\end{tabular}

\section{Population growth}

Kruskal-Wallis test comparing the population growth of cultures exposed to control temperatures or a slow temperature increase, under an ambient, imbalanced or enriched nutrient regime (six treatment groups total)

\begin{tabular}{|c|c|c|c|}
\hline Total N & df & Test statistic & p-value \\
\hline 53 & 5 & 45.48 & $<0.001$ \\
\hline
\end{tabular}

Dunn's multiple comparison test of all possible pairwise comparisons between the population growth rate of cultures exposed to control temperatures or a slow temperature increase, under an ambient, imbalanced or enriched nutrient regime (six treatment groups total). $\mathrm{L}=\mathrm{ambient}$ nutrient levels, $\mathrm{HL}=$ imbalanced nutrient levels, $\mathrm{H}=$ enriched nutrient levels, $\mathrm{C}=$ control temperature $\left(25^{\circ} \mathrm{C}\right), \mathrm{T}=$ treatment temperature $\left(34^{\circ} \mathrm{C}\right)$

\begin{tabular}{|c|c|c|}
\hline Comparison & Test statistic & p-value \\
\hline$H L T-H L C$ & 5.778 & $\mathbf{1 . 0 0 0}$ \\
\hline$H L T-L C$ & 14.22 & $\mathbf{1 . 0 0 0}$ \\
\hline$H L T-H C$ & -28.89 & $\mathbf{0 . 0 0 5}$ \\
\hline$H L T-H T$ & -34.17 & $<\mathbf{0 . 0 0 1}$ \\
\hline$H L T-L T$ & 38.56 & $<\mathbf{0 . 0 0 1}$ \\
\hline$H L C-L C$ & 8.444 & $\mathbf{1 . 0 0 0}$ \\
\hline$H L C-H C$ & -21.11 & $\mathbf{0 . 0 7 8}$ \\
\hline$H L C-H T$ & -28.39 & $\mathbf{0 . 0 0 3}$ \\
\hline
\end{tabular}




\begin{tabular}{|c|c|c|}
\hline$H L C-L T$ & 32.78 & $<\mathbf{0 . 0 0 1}$ \\
\hline$L C-H C$ & -12.67 & $\mathbf{1 . 0 0 0}$ \\
\hline$L C-H T$ & -19.94 & $\mathbf{0 . 1 6 5}$ \\
\hline$L C-L T$ & -24.33 & $\mathbf{0 . 0 1 7}$ \\
\hline$H C-H T$ & -7.278 & $\mathbf{1 . 0 0 0}$ \\
\hline$H C-L T$ & 11.67 & $\mathbf{1 . 0 0 0}$ \\
\hline$H T-L T$ & 4.389 & $\mathbf{1 . 0 0 0}$ \\
\hline
\end{tabular}

\section{A2.2. Appendices for proteomic analysis}

Permutational multivariate analysis of variance

Results of a Permutational multivariate analysis of variance (PERMANOVA) of the entire proteomics data set

\begin{tabular}{|c|c|c|c|c|c|}
\hline & $\begin{array}{c}\text { Degrees of } \\
\text { freedom }\end{array}$ & $\begin{array}{c}\text { Sum of } \\
\text { squares }\end{array}$ & $\mathbf{R}^{\mathbf{2}}$ & F statistic & P-value \\
\hline Temperature & 1 & $4.523 \mathrm{e}^{-4}$ & 0.103 & 3.958 & $\mathbf{0 . 0 0 1}$ \\
\hline Nutrient regime & 2 & $6.510 \mathrm{e}^{-4}$ & 0.148 & 2.848 & $\mathbf{0 . 0 0 1}$ \\
\hline Interaction & 2 & $2.006 \mathrm{e}^{-4}$ & 0.046 & 0.878 & $\mathbf{0 . 6 6 7}$ \\
\hline Residual & 27 & 0.003 & 0.703 & & \\
\hline Total & 32 & 0.004 & 1.000 & & \\
\hline
\end{tabular}

\section{Tables of differentially abundant proteins between treatments}

Table S1: Differentially abundant proteins between cultures at $25^{\circ} \mathrm{C}$ and $34^{\circ} \mathrm{C}$ based on $\log _{2}$ fold change and a student's t-test ( $p$-value presented). Orange $=$ proteins with a greater abundance at $34^{\circ} \mathrm{C}$ relative to $25^{\circ} \mathrm{C}$. Blue $=$ proteins that are depleted at $34^{\circ} \mathrm{C}$ relative to $25^{\circ} \mathrm{C}$.

\begin{tabular}{|cc|ccc|}
\hline Genbank Accession & $\begin{array}{c}\text { Fold } \\
\text { change } \\
\left(\mathbf{l o g}_{2}\right)\end{array}$ & p-value & Annotation & $\begin{array}{c}\text { Uniprot KB } \\
\text { Accession }\end{array}$ \\
\hline $\begin{array}{c}\text { Photosynthesis } \\
\text { tr|U6EFR9|U6EFR9_9 } \\
\text { DINO }\end{array}$ & -1.1 & $1.2 \mathrm{E}-02$ & $\begin{array}{c}\text { Photosystem II chlorophyll- } \\
\text { binding protein CP47 }\end{array}$ & A2T359 \\
\hline $\begin{array}{c}\text { tr|U6EFN7|U6EFN7_9 } \\
\text { DINO }\end{array}$ & -1.1 & $1.2 \mathrm{E}-05$ & $\begin{array}{c}\text { Photosystem II CP43 reaction } \\
\text { center protein }\end{array}$ & Q9TM46 \\
\hline $\begin{array}{c}\text { tr|U6EGF5|U6EGF5_9 } \\
\text { DINO }\end{array}$ & -1.0 & $3.6 \mathrm{E}-04$ & Photosystem II protein D1 & Q9MSC2 \\
\hline
\end{tabular}




\begin{tabular}{|c|c|c|c|c|}
\hline \\
\hline $\begin{array}{c}\text { tr|U6EFV0|U6EFV0_9 } \\
\text { DINO }\end{array}$ & -0.9 & $5.5 \mathrm{E}-07$ & Photosytem II D2 protein & АОТОТ0 \\
\hline $\begin{array}{l}\operatorname{tr|A0A1Q9DK91|A0A1~} \\
\text { Q9DK91_SYMMI }\end{array}$ & -0.7 & $1.4 \mathrm{E}-02$ & Carbonic anhydrase 2 & A0R566 \\
\hline $\begin{array}{l}\operatorname{tr} \mid \text { A0A1Q9CDT0|A0A1 } \\
\text { Q9CDT0_SYMMI }\end{array}$ & -0.7 & $6.2 \mathrm{E}-06$ & Apocytochrome f & Q9TKZ1 \\
\hline $\begin{array}{l}\operatorname{tr} \mid \text { A0A1Q9CAL9|A0A1 } \\
\text { Q9CAL9_SYMMI }\end{array}$ & -0.6 & $1.9 \mathrm{E}-03$ & $\begin{array}{l}\text { Cytochrome b6-f complex } \\
\text { iron-sulfur subunit, } \\
\text { chloroplastic }\end{array}$ & $\mathrm{B} 2 \mathrm{~J} 3 \mathrm{~K} 2$ \\
\hline $\begin{array}{c}\operatorname{tr} \mid \text { A0A1Q9C939|A0A1 } \\
\text { Q9C939_SYMMI }\end{array}$ & -0.5 & $9.6 \mathrm{E}-03$ & $\begin{array}{l}\text { Mg-protoporphyrin IX } \\
\text { chelatase }\end{array}$ & P58571 \\
\hline $\begin{array}{l}\operatorname{tr} \mid \text { A0A1Q9DJH5|A0A1 } \\
\text { Q9DJH5_SYMMI }\end{array}$ & -0.5 & $8.6 \mathrm{E}-05$ & $\begin{array}{l}\text { Photosystem I reaction center } \\
\text { subunit II }\end{array}$ & P49481 \\
\hline $\begin{array}{l}\operatorname{tr} \mid \text { A0A1Q9CRG1|A0A1 } \\
\text { Q9CRG1_SYMMI }\end{array}$ & -0.5 & $1.9 \mathrm{E}-04$ & $\begin{array}{c}\text { Fucoxanthin-chlorophyll a-c } \\
\text { binding protein } \mathrm{F}, \\
\text { chloroplastic }\end{array}$ & Q39709 \\
\hline $\begin{array}{l}\operatorname{tr|A0A1Q9C5M9|A0A1~} \\
\text { Q9C5M9_SYMMI }\end{array}$ & -0.5 & $1.3 \mathrm{E}-05$ & $\begin{array}{c}\text { Fucoxanthin-chlorophyll a-c } \\
\text { binding protein A, } \\
\text { chloroplastic }\end{array}$ & Q42395 \\
\hline $\begin{array}{l}\operatorname{tr|A0A1Q9CHC6|A0A1~} \\
\text { Q9CHC6_SYMMI }\end{array}$ & -0.5 & $2.6 \mathrm{E}-04$ & $\begin{array}{c}\text { Photosystem I reaction center } \\
\text { subunit XI }\end{array}$ & Q85FP8 \\
\hline $\begin{array}{l}\operatorname{tr|A0A1Q9D9X1|A0A1~} \\
\text { Q9D9X1_SYMMI }\end{array}$ & -0.4 & 9.9E-04 & Carbonic anhydrase 2 & P9WPJ8 \\
\hline $\begin{array}{c}\operatorname{tr} \mid \text { A0A1Q9CM18|A0A1 } \\
\text { Q9CM18_SYMMI }\end{array}$ & -0.4 & $1.7 \mathrm{E}-03$ & $\begin{array}{c}\text { Fucoxanthin-chlorophyll a-c } \\
\text { binding protein } \mathrm{F}, \\
\text { chloroplastic }\end{array}$ & Q40300 \\
\hline $\begin{array}{c}\operatorname{tr|A0A1Q9DYG4|A0A~} \\
\text { 1Q9DYG4_SYMMI }\end{array}$ & -0.4 & $1.3 \mathrm{E}-03$ & $\begin{array}{c}\text { Fucoxanthin-chlorophyll a-c } \\
\text { binding protein } \mathrm{B}, \\
\text { chloroplastic }\end{array}$ & Q40296 \\
\hline \multicolumn{5}{|l|}{$\begin{array}{l}\text { PS11 repair/ } \\
\text { stabilisation }\end{array}$} \\
\hline $\begin{array}{l}\operatorname{tr} \mid \text { A0A1Q9EK91|A0A1 } \\
\text { Q9EK91_SYMMI }\end{array}$ & -0.6 & $3.1 \mathrm{E}-04$ & $\begin{array}{l}\text { Peptidyl-prolyl cis-trans } \\
\text { isomerase CYP38, } \\
\text { chloroplastic }\end{array}$ & Q9SSA5 \\
\hline $\begin{array}{l}\operatorname{tr|A0A1Q9EXF7|A0A1~} \\
\text { Q9EXF7_SYMMI }\end{array}$ & -0.4 & $1.2 \mathrm{E}-03$ & $\begin{array}{l}\text { ATP-dependent zinc } \\
\text { metalloprotease FtsH }\end{array}$ & O78516 \\
\hline \multicolumn{5}{|l|}{$\begin{array}{c}\text { Mitochondrial electron } \\
\text { transport }\end{array}$} \\
\hline $\begin{array}{l}\operatorname{tr} \mid \text { A0A1Q9E092|A0A1 } \\
\text { Q9E092_SYMMI }\end{array}$ & -1.6 & $6.8 \mathrm{E}-07$ & $\begin{array}{c}\text { External alternative } \\
\text { NAD }(\mathrm{P}) \mathrm{H} \text {-ubiquinone } \\
\text { oxidoreductase B1, } \\
\text { mitochondrial }\end{array}$ & Q9SKT7 \\
\hline $\begin{array}{l}\operatorname{tr} \mid \text { A0A1Q9DIS8|A0A1 } \\
\text { Q9DIS8_SYMMI }\end{array}$ & -0.8 & $2.4 \mathrm{E}-06$ & Cytochrome b2, mitochondrial & P09437 \\
\hline $\begin{array}{l}\operatorname{tr} \mid \text { A0A1Q9E6L1|A0A1 } \\
\text { Q9E6L1_SYMMI }\end{array}$ & -0.7 & $1.1 \mathrm{E}-02$ & $\begin{array}{l}\text { Cytochrome c oxidase subunit } \\
5 \mathrm{~b}-1, \text { mitochondrial }\end{array}$ & Q9LW15 \\
\hline $\begin{array}{l}\operatorname{tr}|A 0 A 1 Q 9 D B 51| A 0 A 1 \\
\text { Q9DB51_SYMMI }\end{array}$ & -0.7 & $3.2 \mathrm{E}-03$ & Cytochrome b2, mitochondrial & P09437 \\
\hline Glycolysis & & & & \\
\hline
\end{tabular}




\begin{tabular}{|c|c|c|c|c|}
\hline $\begin{array}{l}\operatorname{tr|A0A1Q9BUT7|A0A1~} \\
\text { Q9BUT7_SYMMI }\end{array}$ & -0.8 & $8.9 \mathrm{E}-05$ & $\begin{array}{l}\text { Fructose-bisphosphate } \\
\text { aldolase class } 2 \text { (Fragment) }\end{array}$ & O52402 \\
\hline $\begin{array}{l}\operatorname{tr|A0A1Q9CYF7|A0A1~} \\
\text { Q9CYF7_SYMMI }\end{array}$ & -0.6 & $1.8 \mathrm{E}-07$ & Gamma-enolase & P17183 \\
\hline $\begin{array}{l}\operatorname{tr} \mid \text { A0A1Q9EQ13|A0A1 } \\
\text { Q9EQ13_SYMMI }\end{array}$ & -0.5 & $8.4 \mathrm{E}-05$ & $\begin{array}{l}\text { Fructose-bisphosphate } \\
\text { aldolase }\end{array}$ & Q0PAS0 \\
\hline $\begin{array}{l}\operatorname{tr}|A 0 A 1 Q 9 \bar{C} L M 3| A 0 A \\
\text { 1Q9CLM3_SYMMI }\end{array}$ & -0.4 & $8.6 \mathrm{E}-03$ & $\begin{array}{l}\text { Pyruvate dehydrogenase E1 } \\
\text { component }\end{array}$ & Q59637 \\
\hline $\begin{array}{l}\operatorname{tr|A0A1Q9DK79|A0A1~} \\
\text { Q9DK79_SYMMI }\end{array}$ & -0.4 & $1.9 \mathrm{E}-03$ & Enolase & Q8IJN7 \\
\hline $\begin{array}{l}\operatorname{tr|A0A1Q9DPN0|A0A1~} \\
\text { Q9DPN0_SYMMI }\end{array}$ & 0.6 & 4.7E-05 & $\begin{array}{l}\text { Fructose-bisphosphate } \\
\text { aldolase, Cystol }\end{array}$ & P07764 \\
\hline \multicolumn{5}{|l|}{$\begin{array}{l}\text { Carbohydrate } \\
\text { metabolic process }\end{array}$} \\
\hline $\begin{array}{l}\operatorname{tr|A0A1Q9ENC3|A0A1~} \\
\text { Q9ENC3_SYMMI }\end{array}$ & -1.1 & 7.1E-05 & $\begin{array}{l}\text { Putative 1,4-beta-D-glucan } \\
\text { cellobiohydrolase B }\end{array}$ & Q0CMT2 \\
\hline $\begin{array}{c}\operatorname{tr}|A 0 A 1 Q 9 E 889| A 0 A 1 \\
\text { Q9E889_SYMMI }\end{array}$ & -0.7 & $2.1 \mathrm{E}-04$ & $\begin{array}{l}\text { Alpha- } 1,4 \text { glucan } \\
\text { phosphorylase }\end{array}$ & Q00766 \\
\hline $\begin{array}{l}\text { tr|A0A1Q9EPM6|A0A1 } \\
\text { Q9EPM6_SYMMI }\end{array}$ & -0.6 & $2.4 \mathrm{E}-04$ & $\begin{array}{l}\text { 1,3-beta-glucanosyltransferase } \\
\text { GAS2 }\end{array}$ & Q06135 \\
\hline $\begin{array}{l}\operatorname{tr} \mid \text { A0A1Q9CX33|A0A1 } \\
\text { Q9CX33_SYMMI }\end{array}$ & -0.6 & 8.4E-03 & $\begin{array}{l}\text { Ribulose-phosphate } 3 \text { - } \\
\text { epimerase, chloroplastic }\end{array}$ & Q43157 \\
\hline \multicolumn{5}{|l|}{ Calvin cycle } \\
\hline $\begin{array}{l}\operatorname{tr|A0A1Q9D916|A0A1~} \\
\text { Q9D916_SYMMI }\end{array}$ & -0.9 & 2.7E-03 & Phosphoribulokinase & P26302 \\
\hline $\begin{array}{c}\operatorname{tr|A0A1Q9CYG9|A0A~} \\
\text { 1Q9CYG9_SYMMI }\end{array}$ & -0.6 & $3.2 \mathrm{E}-03$ & $\begin{array}{c}\text { Fructose-1,6-bisphosphatase, } \\
\text { chloroplastic }\end{array}$ & Q07204 \\
\hline $\begin{array}{l}\operatorname{tr|A0A1Q9DR48|A0A1~} \\
\text { Q9DR48_SYMMI }\end{array}$ & -0.5 & $5.8 \mathrm{E}-07$ & $\begin{array}{l}\text { Ribulose bisphosphate } \\
\text { carboxylase }\end{array}$ & Q41406 \\
\hline $\begin{array}{l}\operatorname{tr}|A 0 A 1 Q 9 C C W 0| A 0 A \\
\text { 1Q9CCW0_SYMMI }\end{array}$ & -0.4 & $3.0 \mathrm{E}-03$ & $\begin{array}{l}\text { Glyceraldehyde-3-phosphate } \\
\text { dehydrogenase, glycosomal }\end{array}$ & P22512 \\
\hline $\begin{array}{l}\operatorname{tr}|A 0 A 1 Q 9 E 398| A 0 A 1 \\
\text { Q9E398_SYMMI }\end{array}$ & -0.3 & $6.9 \mathrm{E}-05$ & $\begin{array}{c}\text { Sedoheptulose-1,7- } \\
\text { bisphosphatase, chloroplastic }\end{array}$ & O20252 \\
\hline \multicolumn{5}{|l|}{ TCA cycle } \\
\hline $\begin{array}{c}\operatorname{tr}|A 0 A 1 Q 9 D 779| A 0 A 1 \\
\text { Q9D779_SYMMI }\end{array}$ & -0.7 & $2.8 \mathrm{E}-06$ & $\begin{array}{c}\text { Phosphoenolpyruvate } \\
\text { carboxylase, housekeeping } \\
\text { isozyme }\end{array}$ & Q02909 \\
\hline $\begin{array}{c}\operatorname{tr|A0A1Q9F2F5|A0A1~} \\
\text { Q9F2F5_SYMMI }\end{array}$ & -0.6 & 4.9E-05 & $\begin{array}{l}\text { Malate dehydrogenase, } \\
\text { mitochondrial }\end{array}$ & Q42686 \\
\hline $\begin{array}{c}\operatorname{tr|A0A1Q9E704|A0A1~} \\
\text { Q9E704_SYMMI }\end{array}$ & -0.5 & $3.6 \mathrm{E}-06$ & ATP-citrate synthase & Q91V92 \\
\hline $\begin{array}{l}\operatorname{tr|A0A1Q9ERZ3|A0A1~} \\
\text { Q9ERZ3_SYMMI }\end{array}$ & -0.4 & $3.4 \mathrm{E}-03$ & $\begin{array}{l}\text { Isocitrate dehydrogenase } \\
\text { [NADP] } 2\end{array}$ & P41561 \\
\hline \multicolumn{5}{|l|}{ Acetyl-CoA synthesis } \\
\hline $\begin{array}{l}\operatorname{tr} \mid \text { A0A1Q9EJJ4|A0A1 } \\
\text { Q9EJJ4_SYMMI }\end{array}$ & 0.9 & $1.7 \mathrm{E}-04$ & Probable acetate kinase & B8DMG5 \\
\hline $\begin{array}{l}\operatorname{tr|A0A1Q9CKW4|A0A~} \\
\text { 1Q9CKW4_SYMMI }\end{array}$ & 0.8 & $5.6 \mathrm{E}-03$ & Chitin deacetylase 1 & A0A1Q9CKW4 \\
\hline
\end{tabular}




\begin{tabular}{|c|c|c|c|c|}
\hline \multicolumn{5}{|c|}{$\begin{array}{c}\text { Acetyl-CoA binding and } \\
\text { desaturation }\end{array}$} \\
\hline $\begin{array}{l}\operatorname{tr|A0A1Q9E2E2|A0A1~} \\
\text { Q9E2E2_SYMMI }\end{array}$ & 0.8 & $2.0 \mathrm{E}-02$ & Acyl-CoA dehydrogenase & Q5ATG5 \\
\hline $\begin{array}{l}\operatorname{tr} \mid \text { A0A1Q9BTU5|A0A1 } \\
\text { Q9BTU5_SYMMI }\end{array}$ & 0.8 & $3.4 \mathrm{E}-02$ & $\begin{array}{l}\text { Putative acyl-CoA-binding } \\
\text { protein }\end{array}$ & Q9Y7Z3 \\
\hline \multicolumn{5}{|l|}{ Fatty acid biosynthesis } \\
\hline $\begin{array}{l}\operatorname{tr|A0A1Q9D014|A0A1~} \\
\text { Q9D014_SYMMI }\end{array}$ & -0.8 & $2.4 \mathrm{E}-04$ & $\begin{array}{c}\text { Enoyl-[acyl-carrier-protein] } \\
\text { reductase [NADH], } \\
\text { chloroplastic }\end{array}$ & Q6Z0I4 \\
\hline $\begin{array}{l}\operatorname{tr} \mid \text { A0A1Q9CFD2|A0A1 } \\
\text { Q9CFD2_SYMMI }\end{array}$ & -0.5 & $2.5 \mathrm{E}-03$ & Acetyl-CoA carboxylase 1 & Q00955 \\
\hline \multicolumn{5}{|l|}{ Biosynthesis } \\
\hline $\begin{array}{l}\operatorname{tr}|\mathrm{A} 0 \mathrm{~A} 1 \mathrm{Q} 9 \mathrm{C} 6 \mathrm{C} 4| \mathrm{A} 0 \mathrm{~A} 1 \\
\text { Q9C6C4_SYMMI }\end{array}$ & -1.2 & $8.6 \mathrm{E}-05$ & $\begin{array}{l}\text { Putative alcohol } \\
\text { dehydrogenase }\end{array}$ & P37686 \\
\hline $\begin{array}{l}\operatorname{tr} \mid \text { A0A1Q9D3N5|A0A1 } \\
\text { Q9D3N5_SYMMI }\end{array}$ & -1.2 & $8.6 \mathrm{E}-05$ & Ketol-acid reductoisomerase & Q11VZ3 \\
\hline $\begin{array}{l}\operatorname{tr} \mid \text { A0A1Q9E7J3|A0A1 } \\
\text { Q9E7J3_SYMMI }\end{array}$ & -0.8 & $1.5 \mathrm{E}-03$ & Adenosine kinase 1 & O49923 \\
\hline $\begin{array}{l}\text { tr|A0A1Q9DDY6|A0A } \\
\text { 1Q9DDY6_SYMMI }\end{array}$ & -0.6 & 7.4E-03 & Methionine synthase & Q8DCJ7 \\
\hline $\begin{array}{l}\operatorname{tr}|A 0 A 1 Q 9 C A X 4| A 0 A \\
\text { 1Q9CAX4_SYMMI }\end{array}$ & -0.6 & $9.8 \mathrm{E}-03$ & Uridine kinase & Q9FKS0 \\
\hline $\begin{array}{c}\operatorname{tr} \mid \text { A0A1Q9EJ86|A0A1 } \\
\text { Q9EJ86_SYMMI }\end{array}$ & -0.4 & $5.1 \mathrm{E}-04$ & $\begin{array}{l}\text { Phosphoserine } \\
\text { aminotransferase }\end{array}$ & Q820S0 \\
\hline $\begin{array}{c}\operatorname{tr|A0A1Q9DGX7|A0A~} \\
\text { 1Q9DGX7_SYMMI }\end{array}$ & 0.9 & $1.5 \mathrm{E}-02$ & Dihydroxy-acid dehydratase & B0TZC0 \\
\hline \multicolumn{5}{|l|}{ RNA binding } \\
\hline $\begin{array}{l}\operatorname{tr|A0A1Q9DRE2|A0A1~} \\
\text { Q9DRE2_SYMMI }\end{array}$ & 1.0 & $1.9 \mathrm{E}-02$ & $\begin{array}{l}\text { MATH and LRR domain- } \\
\text { containing protein }\end{array}$ & Q8I3Z1 \\
\hline $\begin{array}{l}\operatorname{tr|A0A1Q9C394|A0A1~} \\
\text { Q9C394_SYMMI }\end{array}$ & 0.9 & 7.7E-03 & $\begin{array}{l}\text { ATP-dependent RNA helicase } \\
\text { dbp2 }\end{array}$ & Q2H720 \\
\hline $\begin{array}{l}\operatorname{tr|A0A1Q9EW51|A0A1~} \\
\text { Q9EW51_SYMMI }\end{array}$ & 0.4 & 7.1E-04 & $\begin{array}{c}\text { Splicing factor U2af large } \\
\text { subunit B }\end{array}$ & Q9ZR40 \\
\hline \multicolumn{5}{|c|}{$\begin{array}{c}\text { Regulation/repression of } \\
\text { translation }\end{array}$} \\
\hline $\begin{array}{l}\operatorname{tr|A0A1Q9D0J7|A0A1~} \\
\text { Q9D0J7_SYMMI }\end{array}$ & 0.6 & $2.4 \mathrm{E}-03$ & $\begin{array}{l}\text { Pentatricopeptide repeat- } \\
\text { containing protein, } \\
\text { mitochondrial }\end{array}$ & Q9FME4 \\
\hline $\begin{array}{l}\operatorname{tr} \mid \text { A0A1Q9EEL3|A0A1 } \\
\text { Q9EEL3_SYMMI }\end{array}$ & 0.5 & $1.0 \mathrm{E}-02$ & $\begin{array}{l}\text { Eukaryotic translation } \\
\text { initiation factor } 3 \text { subunit } E\end{array}$ & A7RWP6 \\
\hline \multicolumn{5}{|l|}{ Protein translation } \\
\hline $\begin{array}{l}\operatorname{tr|A0A1Q9E984|A0A1~} \\
\text { Q9E984_SYMMI }\end{array}$ & -1.1 & $1.2 \mathrm{E}-06$ & $\begin{array}{l}\text { rRNA adenine } \mathrm{N}(6)- \\
\text { methyltransferase }\end{array}$ & Q2S0I2 \\
\hline $\begin{array}{l}\operatorname{tr} \mid \text { A0A1Q9EQV9|A0A1 } \\
\text { Q9EQV9_SYMMI }\end{array}$ & -0.9 & $1.1 \mathrm{E}-02$ & $\begin{array}{l}\text { Elongation factor Ts, } \\
\text { mitochondrial }\end{array}$ & B1XQQ0 \\
\hline $\begin{array}{l}\operatorname{tr|A0A1Q9E0V8|A0A1~} \\
\text { Q9E0V8_SYMMI }\end{array}$ & -0.8 & $7.8 \mathrm{E}-03$ & $\begin{array}{l}\text { 60S acidic ribosomal protein } \\
\text { P1 }\end{array}$ & P29763 \\
\hline
\end{tabular}




\begin{tabular}{|c|c|c|c|c|}
\hline $\begin{array}{l}\operatorname{tr} \mid \text { A0A1Q9CLC6|A0A1 } \\
\text { Q9CLC6_SYMMI }\end{array}$ & -0.7 & $2.6 \mathrm{E}-02$ & 30 S ribosomal protein $\mathrm{S} 2$ & O67809 \\
\hline $\begin{array}{l}\operatorname{tr|A0A1Q9CED7|A0A1~} \\
\text { Q9CED7_SYMMI }\end{array}$ & -0.7 & $2.5 \mathrm{E}-02$ & $\begin{array}{l}\text { Ribosomal RNA small subunit } \\
\text { methyltransferase F }\end{array}$ & A8AFL6 \\
\hline $\begin{array}{l}\operatorname{tr}|A 0 A 1 Q 9 D K U 8| A 0 A \\
\text { 1Q9DKU8_SYMMI }\end{array}$ & -0.5 & 7.1E-03 & Endoglucanase & P10475 \\
\hline $\begin{array}{l}\operatorname{tr|A0A1Q9DLA5|A0A1~} \\
\text { Q9DLA5_SYMMI }\end{array}$ & -0.5 & $2.4 \mathrm{E}-03$ & 60 S ribosomal protein $\mathrm{L} 7-4$ & Q6C603 \\
\hline $\begin{array}{l}\operatorname{tr|A0A1Q9D0D0|A0A1~} \\
\text { Q9D0D0_SYMMI }\end{array}$ & -0.5 & $1.0 \mathrm{E}-02$ & 40S ribosomal protein $\mathrm{S} 8$ & Q4N3P0 \\
\hline $\begin{array}{l}\operatorname{tr} \mid \text { A0A1Q9CNF2|A0A1 } \\
\text { Q9CNF2_SYMMI }\end{array}$ & 1.1 & $4.2 \mathrm{E}-02$ & Elongation factor Tu-B & Q4URD7 \\
\hline \multicolumn{5}{|l|}{$\begin{array}{c}\text { Protein } \\
\text { sorting/transport }\end{array}$} \\
\hline $\begin{array}{l}\operatorname{tr} \mid \text { A0A1Q9CN28|A0A1 } \\
\text { Q9CN28_SYMMI }\end{array}$ & -1.3 & $4.5 \mathrm{E}-04$ & $\begin{array}{l}\text { Brefeldin A-inhibited guanine } \\
\text { nucleotide-exchange protein } 2\end{array}$ & Q9LZX8 \\
\hline $\begin{array}{l}\operatorname{tr|A0A1Q9EXN6|A0A1~} \\
\text { Q9EXN6_SYMMI }\end{array}$ & 1.1 & $8.6 \mathrm{E}-03$ & Ras-related protein Rab-34 & Q9TVU5 \\
\hline $\begin{array}{l}\operatorname{tr} \mid \text { A0A1Q9CGS1|A0A1 } \\
\text { Q9CGS1_SYMMI }\end{array}$ & 0.7 & $3.8 \mathrm{E}-04$ & Protein transport protein sec 31 & A1DHK2 \\
\hline $\begin{array}{c}\operatorname{tr}|\mathrm{A} 0 \mathrm{~A} 1 \mathrm{Q} 9 \mathrm{C} 2 \mathrm{~F} 0| \mathrm{A} 0 \mathrm{~A} 1 \\
\text { Q9C2F0_SYMMI }\end{array}$ & 0.6 & $1.6 \mathrm{E}-02$ & GTP-binding protein SAR1A & O04834 \\
\hline \multicolumn{5}{|l|}{ Protein kinase activity } \\
\hline $\begin{array}{l}\operatorname{tr} \mid \text { A0A1Q9D050|A0A1 } \\
\text { Q9D050_SYMMI }\end{array}$ & -3.2 & $4.8 \mathrm{E}-08$ & $\begin{array}{l}\text { Stress-activated protein kinase } \\
\text { JNK }\end{array}$ & Q9N272 \\
\hline $\begin{array}{l}\operatorname{tr|A0A1Q9EMH4|A0A~} \\
\text { 1Q9EMH4_SYMMI }\end{array}$ & -0.9 & 7.7E-03 & $\begin{array}{l}\text { Putative peptidase C1-like } \\
\text { protein }\end{array}$ & Q5UQE9 \\
\hline $\begin{array}{l}\operatorname{tr|A0A1Q9DA97|A0A1~} \\
\text { Q9DA97_SYMMI }\end{array}$ & -0.8 & $6.1 \mathrm{E}-03$ & $\begin{array}{c}\text { Serine/threonine-protein } \\
\text { kinase rio2 }\end{array}$ & Q54T05 \\
\hline $\begin{array}{l}\operatorname{tr}|\mathrm{A} 0 \mathrm{~A} 1 \mathrm{Q} 9 \mathrm{CDH} 4| \mathrm{A} 0 \mathrm{~A} \\
\text { 1Q9CDH4_SYMMI }\end{array}$ & 0.9 & $3.1 \mathrm{E}-05$ & Pyruvate, phosphate dikinase & Q59754 \\
\hline \multicolumn{5}{|l|}{ Nitrogen metabolism } \\
\hline $\begin{array}{l}\operatorname{tr}|A 0 A 1 Q 9 D I 38| A 0 A 1 \\
\text { Q9DI38_SYMMI }\end{array}$ & -0.8 & $2.1 \mathrm{E}-02$ & Type-3 glutamine synthetase & Q54WR9 \\
\hline $\begin{array}{l}\operatorname{tr}|\mathrm{A} 0 \mathrm{~A} 1 \mathrm{Q} 9 \mathrm{E} 3 \mathrm{~K} 5| \mathrm{A} 0 \mathrm{~A} 1 \\
\text { Q9E3K5_SYMMI }\end{array}$ & -0.6 & $5.4 \mathrm{E}-03$ & Type-3 glutamine synthetase & Q54WR9 \\
\hline $\begin{array}{l}\operatorname{tr}|\mathrm{A} 0 \mathrm{~A} 1 \mathrm{Q} 9 \mathrm{C} 7 \mathrm{P} 0| \mathrm{A} 0 \mathrm{~A} 1 \\
\text { Q9C7P0_SYMMI }\end{array}$ & 0.5 & 7.7E-03 & $\begin{array}{c}\text { Putative methyltransferase, } \\
\text { chloroplastic }\end{array}$ & Q0WPT7 \\
\hline \multicolumn{5}{|l|}{ Membrane transport } \\
\hline $\begin{array}{l}\operatorname{tr} \mid \text { A0A1Q9EBH2|A0A1 } \\
\text { Q9EBH2_SYMMI }\end{array}$ & -0.8 & 4.8E-03 & $\mathrm{Na}(+) / \mathrm{H}(+)$ antiporter $\mathrm{NhaD}$ & Q9C6D3 \\
\hline $\begin{array}{l}\operatorname{tr|A0A1Q9D817|A0A1~} \\
\text { Q9D817_SYMMI }\end{array}$ & -0.6 & $3.3 \mathrm{E}-03$ & Plasma membrane ATPase & Q58623 \\
\hline $\begin{array}{l}\operatorname{tr} \mid \text { A0A1Q9F016|A0A1 } \\
\text { Q9F016_SYMMI }\end{array}$ & -0.4 & $6.5 \mathrm{E}-04$ & $\begin{array}{c}\text { Electrogenic sodium } \\
\text { bicarbonate cotransporter } 1\end{array}$ & Q9Y6R1 \\
\hline $\begin{array}{c}\operatorname{tr} \mid \text { A0A1Q9C990|A0A1 } \\
\text { Q9C990_SYMMI }\end{array}$ & 0.8 & 3.7E-02 & $\begin{array}{l}\text { V-type proton ATPase subunit } \\
\text { F }\end{array}$ & Q55AH5 \\
\hline Proteolysis & & & & \\
\hline
\end{tabular}




\begin{tabular}{|c|c|c|c|c|}
\hline $\begin{array}{c}\operatorname{tr|A0A1Q9DNG3|A0A~} \\
\text { 1Q9DNG3_SYMMI }\end{array}$ & -0.6 & $1.7 \mathrm{E}-02$ & $\begin{array}{c}\text { Cathepsin B-like cysteine } \\
\text { proteinase } 6\end{array}$ & P43510 \\
\hline $\begin{array}{l}\operatorname{tr|A0A1Q9DCF4|A0A1~} \\
\text { Q9DCF4_SYMMI }\end{array}$ & -0.6 & $3.7 \mathrm{E}-03$ & Oryzain alpha chain & P25776 \\
\hline $\begin{array}{l}\operatorname{tr} \mid \text { A0A1Q9DPK3|A0A1 } \\
\text { Q9DPK3_SYMMI }\end{array}$ & -0.6 & $2.5 \mathrm{E}-03$ & $\begin{array}{l}\text { Pregnancy-associated } \\
\text { glycoprotein }\end{array}$ & Q28389 \\
\hline $\begin{array}{l}\operatorname{tr|A0A1Q9CV48|A0A1~} \\
\text { Q9CV48_SYMMI }\end{array}$ & -0.5 & 8.7E-03 & Xylem cysteine proteinase 1 & O65493 \\
\hline \multicolumn{5}{|l|}{ Cell division } \\
\hline $\begin{array}{l}\operatorname{tr}|A 0 A 1 Q 9 E X I 4| A 0 A 1 \\
\text { Q9EXI4_SYMMI }\end{array}$ & -1.6 & 8.9E-06 & $\begin{array}{l}\text { Peptidyl-prolyl cis-trans } \\
\text { isomerase }\end{array}$ & P52014 \\
\hline $\begin{array}{l}\operatorname{tr|A0A1Q9DA21|A0A1~} \\
\text { Q9DA21_SYMMI }\end{array}$ & -0.5 & 7.0E-03 & Actin & P53476 \\
\hline $\begin{array}{l}\operatorname{tr|A0A1Q9D6Y6|A0A1~} \\
\text { Q9D6Y6_SYMMI }\end{array}$ & -0.5 & $6.0 \mathrm{E}-05$ & $\begin{array}{l}\text { Cyclin-dependent kinases } \\
\text { regulatory subunit }\end{array}$ & Q40300 \\
\hline \multicolumn{5}{|l|}{ Protein folding } \\
\hline $\begin{array}{c}\operatorname{tr|A0A1Q9DGC0|A0A~} \\
\text { 1Q9DGC0_SYMMI }\end{array}$ & 2.2 & $1.3 \mathrm{E}-08$ & Peptidylprolyl isomerase & Q9FJL3 \\
\hline $\begin{array}{l}\operatorname{tr} \mid \text { A0A1Q9E4G2|A0A1 } \\
\text { Q9E4G2_SYMMI }\end{array}$ & 2.0 & $7.1 \mathrm{E}-07$ & Chaperone protein DnaK & A7HZ39 \\
\hline $\begin{array}{l}\operatorname{tr|A0A1Q9C9B4|A0A1~} \\
\text { Q9C9B4_SYMMI }\end{array}$ & 1.9 & $5.2 \mathrm{E}-07$ & $60 \mathrm{kDa}$ chaperonin 1 & Q05972 \\
\hline $\begin{array}{c}\operatorname{tr} \mid \text { A0A1Q9F4T9|A0A1 } \\
\text { Q9F4T9_SYMMI }\end{array}$ & 1.3 & $1.4 \mathrm{E}-04$ & Chaperone protein ClpB & Q8DJ40 \\
\hline $\begin{array}{l}\operatorname{tr} \mid \text { A0A1Q9EC13|A0A1 } \\
\text { Q9EC13_SYMMI }\end{array}$ & 1.2 & $1.1 \mathrm{E}-06$ & $\begin{array}{l}\text { Heat shock cognate } 90 \mathrm{kDa} \\
\text { protein }\end{array}$ & Q69QQ6 \\
\hline $\begin{array}{l}\operatorname{tr} \mid \text { A0A1Q9C5U7|A0A1 } \\
\text { Q9C5U7_SYMMI }\end{array}$ & 1.2 & $1.2 \mathrm{E}-06$ & $\begin{array}{l}20 \text { kDa chaperonin, } \\
\text { chloroplastic }\end{array}$ & O65282 \\
\hline $\begin{array}{l}\operatorname{tr|A0A1Q9DLJ1|A0A1~} \\
\text { Q9DLJ1_SYMMI }\end{array}$ & 1.1 & $2.1 \mathrm{E}-07$ & $\begin{array}{l}\text { Chaperonin CPN60-2, } \\
\text { mitochondrial }\end{array}$ & Q05045 \\
\hline $\begin{array}{c}\operatorname{tr|A0A1Q9CQB8|A0A1~} \\
\text { Q9CQB8_SYMMI }\end{array}$ & 1.1 & $5.5 \mathrm{E}-08$ & $60 \mathrm{kDa}$ chaperonin 1 & B0CFQ6 \\
\hline $\begin{array}{l}\operatorname{tr|A0A1Q9F1M0|A0A1~} \\
\text { Q9F1M0_SYMMI }\end{array}$ & 1.1 & $3.1 \mathrm{E}-03$ & Peptidylprolyl isomerase & O04287 \\
\hline $\begin{array}{l}\operatorname{tr} \mid \text { A0A1Q9EA76|A0A1 } \\
\text { Q9EA76_SYMMI }\end{array}$ & 1.1 & $1.1 \mathrm{E}-05$ & Protein GrpE & Q2JH51 \\
\hline $\begin{array}{l}\operatorname{tr} \mid \text { A0A1Q9C0C1|A0A1 } \\
\text { Q9C0C1_SYMMI }\end{array}$ & 1.0 & $5.5 \mathrm{E}-06$ & $\begin{array}{l}60 \text { kDa chaperonin } 1 \\
\text { (Fragment) }\end{array}$ & A5ECI7 \\
\hline $\begin{array}{l}\operatorname{tr|A0A1Q9F4W2|A0A1~} \\
\text { Q9F4W2_SYMMI }\end{array}$ & 1.0 & $5.9 \mathrm{E}-05$ & Chaperone protein $\mathrm{ClpB} 2$ & Q8DJ40 \\
\hline $\begin{array}{l}\operatorname{tr|A0A1Q9DD82|A0A1~} \\
\text { Q9DD82_SYMMI }\end{array}$ & 1.0 & $1.0 \mathrm{E}-05$ & $\begin{array}{l}\text { Chaperonin CPN60-2, } \\
\text { mitochondrial (Fragment) }\end{array}$ & P29185 \\
\hline $\begin{array}{c}\operatorname{tr|A0A1Q9DNB8|A0A~} \\
\text { 1Q9DNB8_SYMMI }\end{array}$ & 0.9 & $3.4 \mathrm{E}-04$ & Chaperone protein ClpB1 & P42730 \\
\hline $\begin{array}{l}\operatorname{tr|A0A1Q9DYJ8|A0A1~} \\
\text { Q9DYJ8_SYMMI }\end{array}$ & 0.9 & $2.0 \mathrm{E}-07$ & Chaperone protein DnaK & Q11KJ6 \\
\hline $\begin{array}{l}\operatorname{tr} \mid \text { A0A1Q9CJK1|A0A1 } \\
\text { Q9CJK1_SYMMI }\end{array}$ & 0.9 & $3.2 \mathrm{E}-08$ & $\begin{array}{l}\text { Chaperone protein dnaK2 } \\
\text { (Fragment) }\end{array}$ & Q7U3C4 \\
\hline
\end{tabular}




\begin{tabular}{|c|c|c|c|c|}
\hline $\begin{array}{l}\operatorname{tr} \mid \text { A0A1Q9CRD2|A0A1 } \\
\text { Q9CRD2_SYMMI }\end{array}$ & 0.9 & 4.9E-06 & Heat shock protein 90 & O44001 \\
\hline $\begin{array}{c}\operatorname{tr|A0A1Q9CYK7|A0A~} \\
\text { 1Q9CYK7_SYMMI }\end{array}$ & 0.8 & $3.0 \mathrm{E}-03$ & STI1-like protein & Q8ILC1 \\
\hline $\begin{array}{l}\operatorname{tr} \mid \text { A0A1Q9CBC6|A0A1 } \\
\text { Q9CBC6_SYMMI }\end{array}$ & 0.7 & 2.2E-06 & Heat shock $70 \mathrm{kDa}$ protein & P11144 \\
\hline $\begin{array}{l}\operatorname{tr} \mid \text { A0A1Q9EUD6|A0A1 } \\
\text { Q9EUD6_SYMMI }\end{array}$ & 0.6 & $1.0 \mathrm{E}-02$ & Catechol & O88587 \\
\hline $\begin{array}{l}\operatorname{tr} \mid \text { A0A1Q9CC83|A0A1 } \\
\text { Q9CC83_SYMMI }\end{array}$ & 0.6 & $5.0 \mathrm{E}-03$ & Prohibitin-2 & P50085 \\
\hline $\begin{array}{l}\operatorname{tr|A0A1Q9BSY4|A0A1~} \\
\text { Q9BSY4_SYMMI }\end{array}$ & 0.5 & 2.7E-04 & Endoplasmin-like (Fragment) & Q9STX5 \\
\hline $\begin{array}{l}\operatorname{tr}|A 0 A 1 Q 9 D F 44| A 0 A 1 \\
\text { Q9DF44_SYMMI }\end{array}$ & 0.4 & $1.1 \mathrm{E}-03$ & Calreticulin & P27797 \\
\hline \multicolumn{5}{|c|}{$\begin{array}{c}\text { Redox homeostasis/oxidative stress } \\
\text { response }\end{array}$} \\
\hline $\begin{array}{l}\operatorname{tr} \mid \text { A0A1Q9DIE8|A0A1 } \\
\text { Q9DIE8_SYMMI }\end{array}$ & 0.9 & 1.1E-03 & $\begin{array}{l}\text { Glutaredoxin-related protein } \\
5, \text { mitochondrial }\end{array}$ & Q80Y14 \\
\hline $\begin{array}{l}\operatorname{tr} \mid \text { A0A1Q9DKA2|A0A } \\
\text { 1Q9DKA2_SYMMI }\end{array}$ & 0.7 & $2.4 \mathrm{E}-02$ & $\begin{array}{c}\text { Putative NADPH:quinone } \\
\text { oxidoreductase } 1\end{array}$ & Q92R45 \\
\hline $\begin{array}{l}\operatorname{tr} \mid \text { A0A1Q9EV03|A0A1 } \\
\text { Q9EV03_SYMMI }\end{array}$ & 0.6 & $2.1 \mathrm{E}-03$ & $\begin{array}{l}\text { Quinone-oxidoreductase-like, } \\
\text { chloroplastic }\end{array}$ & Q9AYU1 \\
\hline \multicolumn{5}{|l|}{ Cell wall organisation } \\
\hline $\begin{array}{c}\operatorname{tr}|A 0 A 1 Q 9 C W B 5| A 0 A \\
\text { 1Q9CWB5_SYMMI }\end{array}$ & 0.5 & 1.7E-03 & $\begin{array}{c}\text { Putative glucan 1,3-beta- } \\
\text { glucosidase A }\end{array}$ & B0XN12 \\
\hline \multicolumn{5}{|l|}{$\begin{array}{l}\text { DNA integration/ } \\
\text { recombination }\end{array}$} \\
\hline $\begin{array}{l}\operatorname{tr|A0A1Q9EBZ7|A0A1~} \\
\text { Q9EBZ7_SYMMI }\end{array}$ & 0.7 & $1.5 \mathrm{E}-02$ & Copia protein & P04146 \\
\hline $\begin{array}{c}\operatorname{tr} \mid \text { A0A1Q9E9N4|A0A1 } \\
\text { Q9E9N4_SYMMI }\end{array}$ & 0.5 & $2.0 \mathrm{E}-03$ & $\begin{array}{c}\text { Retrovirus-related Pol } \\
\text { polyprotein from transposon } \\
\text { TNT 1-94 }\end{array}$ & Q94HW2 \\
\hline
\end{tabular}

Table S2: Differentially abundant proteins between the imbalanced and enriched nutrient regime based on $\log _{2}$ fold change and a student's t-test ( $p$-value presented). Orange= proteins with a greater abundance under an imbalanced nutrient regime relative to the enriched regime. Blue= proteins that a depleted under the imbalanced nutrient regime relative to the enriched regime.

\begin{tabular}{|c|c|c|c|c|}
\hline Genbank Accession & $\begin{array}{l}\text { Fold } \\
\text { change } \\
\left(\log _{2}\right)\end{array}$ & p-value & Annotation & $\begin{array}{l}\text { Uniprot KB } \\
\text { accession }\end{array}$ \\
\hline \multicolumn{5}{|l|}{ RNA binding } \\
\hline $\begin{array}{c}\operatorname{tr|A0A1Q9CC39|A0A1~} \\
\text { Q9CC39_SYMMI }\end{array}$ & 1.2 & $\begin{array}{c}3.0 \mathrm{E}- \\
02\end{array}$ & $\begin{array}{l}\text { Ribonuclease } P \text { protein } \\
\text { subunit p25-like protein }\end{array}$ & Q8N5L8 \\
\hline $\begin{array}{l}\operatorname{tr} \mid \text { A0A1Q9DD85|A0A1 } \\
\text { Q9DD85_SYMMI }\end{array}$ & 1.1 & $\begin{array}{c}7.2 \mathrm{E}- \\
03\end{array}$ & Uncharacterized protein & O49453 \\
\hline $\begin{array}{l}\operatorname{tr} \mid \text { A0A1Q9DAU1|A0A1 } \\
\text { Q9DAU1_SYMMI }\end{array}$ & 1.0 & $\begin{array}{c}2.5 \mathrm{E}- \\
03\end{array}$ & $\begin{array}{c}\text { ATP-dependent RNA helicase } \\
\text { DED1 }\end{array}$ & Q8TFK8 \\
\hline
\end{tabular}




\begin{tabular}{|c|c|c|c|c|}
\hline $\begin{array}{l}\operatorname{tr|A0A1Q9DIM7|A0A1~} \\
\text { Q9DIM7_SYMMI }\end{array}$ & 0.8 & $\begin{array}{c}5.1 \mathrm{E}- \\
03\end{array}$ & $\begin{array}{l}\text { ATP-dependent RNA helicase } \\
\text { SUB2 }\end{array}$ & Q27268 \\
\hline $\begin{array}{l}\operatorname{tr|A0A1Q9EA87|A0A1~} \\
\text { Q9EA87_SYMMI }\end{array}$ & 0.8 & $\begin{array}{c}1.7 \mathrm{E}- \\
03\end{array}$ & $\begin{array}{l}\text { DEAD-box ATP-dependent } \\
\text { RNA helicase } 14\end{array}$ & Q5JKF2 \\
\hline $\begin{array}{l}\operatorname{tr} \mid \text { A0A1Q9ECP3|A0A1 } \\
\text { Q9ECP3_SYMMI }\end{array}$ & 0.5 & $\begin{array}{c}3.0 \mathrm{E}- \\
03\end{array}$ & $\begin{array}{l}\text { Putative ATP-dependent RNA } \\
\text { helicase ddx6 }\end{array}$ & Q54E49 \\
\hline $\begin{array}{c}\operatorname{tr|A0A1Q9DVV4|A0A1~} \\
\text { Q9DVV4_SYMMI }\end{array}$ & 0.4 & $\begin{array}{l}2.6 \mathrm{E}- \\
03\end{array}$ & $\begin{array}{l}\text { Heterogeneous nuclear } \\
\text { ribonucleoprotein U-like } \\
\text { protein } 1\end{array}$ & Q1KMD3b \\
\hline \multicolumn{5}{|l|}{$\begin{array}{c}\text { mRNA splicing, via the } \\
\text { spliceosome }\end{array}$} \\
\hline $\begin{array}{l}\operatorname{tr|A0A1Q9D431|A0A1~} \\
\text { Q9D431_SYMMI }\end{array}$ & 1.3 & $\begin{array}{c}1.3 \mathrm{E}- \\
02\end{array}$ & $\begin{array}{c}\text { Small nuclear } \\
\text { ribonucleoprotein Sm D2 }\end{array}$ & Q3SZF8 \\
\hline $\begin{array}{l}\operatorname{tr}|A 0 A 1 Q 9 C S L 2| A 0 A 1 \\
\text { Q9CSL2_SYMMI }\end{array}$ & 1.3 & $\begin{array}{c}8.0 \mathrm{E}- \\
03\end{array}$ & $\begin{array}{l}\text { Pro-apoptotic serine protease } \\
\text { NMA111 }\end{array}$ & O44437 \\
\hline $\begin{array}{l}\operatorname{tr} \mid \text { A0A1Q9DFN0|A0A1 } \\
\text { Q9DFN0_SYMMI }\end{array}$ & 1.0 & $\begin{array}{c}2.4 \mathrm{E}- \\
02\end{array}$ & Splicing factor 3B subunit 3 & Q1LVE8 \\
\hline $\begin{array}{l}\operatorname{tr} \mid \text { A0A1Q9DM51|A0A1 } \\
\text { Q9DM51_SYMMI }\end{array}$ & 0.8 & $\begin{array}{c}3.8 \mathrm{E}- \\
03\end{array}$ & $\begin{array}{l}\text { Pre-mRNA-processing- } \\
\text { splicing factor } 8\end{array}$ & Q99PV0 \\
\hline $\begin{array}{c}\operatorname{tr|A0A1Q9CQC9|A0A1~} \\
\text { Q9CQC9_SYMMI }\end{array}$ & 0.7 & $\begin{array}{c}8.9 \mathrm{E}- \\
03\end{array}$ & $\begin{array}{c}\text { Putative ATP-dependent RNA } \\
\text { helicase DDX23 }\end{array}$ & P93008 \\
\hline $\begin{array}{l}\operatorname{tr}|A 0 A 1 Q 9 C 7 H 6| A 0 A 1 \\
\text { Q9C7H6_SYMMI }\end{array}$ & 0.6 & $\begin{array}{c}2.6 \mathrm{E}- \\
05\end{array}$ & $\begin{array}{l}116 \text { kDa U5 small nuclear } \\
\text { ribonucleoprotein component }\end{array}$ & Q15029 \\
\hline $\begin{array}{c}\operatorname{tr} \mid \text { A0A1Q9EGX9|A0A1 } \\
\text { Q9EGX9_SYMMI }\end{array}$ & 0.6 & $\begin{array}{c}1.5 \mathrm{E}- \\
03\end{array}$ & $\begin{array}{l}\text { Eukaryotic initiation factor } \\
\qquad 4 \mathrm{~A}-\mathrm{I}\end{array}$ & B5DG42 \\
\hline $\begin{array}{l}\operatorname{tr|A0A1Q9D188|A0A1~} \\
\text { Q9D188_SYMMI }\end{array}$ & 0.5 & $\begin{array}{c}9.6 \mathrm{E}- \\
04\end{array}$ & $\begin{array}{l}\mathrm{U} 2 \text { small nuclear } \\
\text { ribonucleoprotein A }\end{array}$ & P09661 \\
\hline \multicolumn{5}{|l|}{ Translation } \\
\hline $\begin{array}{c}\operatorname{tr} \mid \text { A0A1Q9CVC1|A0A1 } \\
\text { Q9CVC1_SYMMI }\end{array}$ & 1.0 & $\begin{array}{c}1.9 \mathrm{E}- \\
02\end{array}$ & 40 S ribosomal protein $\mathrm{S} 13-1$ & P62302 \\
\hline $\begin{array}{l}\operatorname{tr}|A 0 A 1 Q 9 \bar{C} C F 8| A 0 A 1 \\
\text { Q9CCF8_SYMMI }\end{array}$ & 1.0 & $\begin{array}{c}9.4 \mathrm{E}- \\
04\end{array}$ & 40S ribosomal protein S16-1 & P46293 \\
\hline $\begin{array}{l}\operatorname{tr} \mid \text { A0A1Q9DCU8|A0A1 } \\
\text { Q9DCU8_SYMMI }\end{array}$ & 0.9 & $\begin{array}{c}5.5 \mathrm{E}- \\
03\end{array}$ & 40S ribosomal protein $\mathrm{S} 7$ & Q9ZNS1 \\
\hline $\begin{array}{c}\operatorname{tr|A0A1Q9CKK7|A0A1~} \\
\text { Q9CKK7_SYMMI }\end{array}$ & 0.9 & $\begin{array}{c}3.2 \mathrm{E}- \\
02\end{array}$ & $60 \mathrm{~S}$ ribosomal protein L13-1 & P41128 \\
\hline $\begin{array}{c}\operatorname{tr} \mid \text { A0A1Q9D6I3|A0A1Q } \\
\text { 9D6I3_SYMMI }\end{array}$ & 0.9 & $\begin{array}{c}2.3 \mathrm{E}- \\
02\end{array}$ & 40S ribosomal protein S9-1 & Q9LXG1 \\
\hline $\begin{array}{c}\operatorname{tr} \mid \mathrm{A} 0 \mathrm{~A} 1 \mathrm{Q} 9 \mathrm{EQH1|A0A1} \\
\text { Q9EQH1_SYMMI }\end{array}$ & 0.8 & $\begin{array}{c}2.5 \mathrm{E}- \\
03\end{array}$ & $\begin{array}{l}\text { Putative tRNA (Adenine(37)- } \\
\text { N6)-methyltransferase }\end{array}$ & P44740 \\
\hline $\begin{array}{c}\operatorname{tr|A0A1Q9DAV8|A0A1~} \\
\text { Q9DAV8_SYMMI }\end{array}$ & 0.8 & $\begin{array}{c}9.8 \mathrm{E}- \\
03\end{array}$ & $60 \mathrm{~S}$ ribosomal protein $\mathrm{L} 13 \mathrm{a}$ & P93099 \\
\hline $\begin{array}{l}\operatorname{tr} \mid \text { A0A1Q9CZJ5|A0A1 } \\
\text { Q9CZJ5_SYMMI }\end{array}$ & 0.7 & $\begin{array}{c}1.9 \mathrm{E}- \\
03\end{array}$ & Tyrosine--tRNA ligase & F4HWL4 \\
\hline $\begin{array}{l}\operatorname{tr|A0A1Q9DYA7|A0A1~} \\
\text { Q9DYA7_SYMMI }\end{array}$ & 0.7 & $\begin{array}{c}1.2 \mathrm{E}- \\
02\end{array}$ & $\begin{array}{l}\text { Eukaryotic translation } \\
\text { initiation factor } 3 \text { subunit A }\end{array}$ & Q9LD55 \\
\hline $\begin{array}{l}\operatorname{tr|A0A1Q9DLA5|A0A1~} \\
\text { Q9DLA5_SYMMI }\end{array}$ & 0.6 & $\begin{array}{c}2.9 \mathrm{E}- \\
03\end{array}$ & 60 S ribosomal protein $\mathrm{L} 7-4$ & Q6C603 \\
\hline $\begin{array}{l}\text { tr|A0A1Q9E562|A0A1 } \\
\text { Q9E562_SYMMI }\end{array}$ & 0.6 & $\begin{array}{c}1.6 \mathrm{E}- \\
03\end{array}$ & Elongation factor 1-alpha & P90519 \\
\hline
\end{tabular}




\begin{tabular}{|c|c|c|c|c|}
\hline $\begin{array}{l}\operatorname{tr}|A 0 A 1 Q 9 E 562| A 0 A 1 \\
\text { Q9E562_SYMMI }\end{array}$ & 0.6 & $\begin{array}{c}1.6 \mathrm{E}- \\
03\end{array}$ & Elongation factor 1-alpha & P90519 \\
\hline $\begin{array}{c}\operatorname{tr}|A 0 A 1 Q 9 E 037| A 0 A 1 \\
\text { Q9E037_SYMMI }\end{array}$ & 0.5 & $\begin{array}{c}6.4 \mathrm{E}- \\
03\end{array}$ & $60 S$ ribosomal protein $\mathrm{L} 27 \mathrm{a}$ & Q00454 \\
\hline $\begin{array}{c}\operatorname{tr} \mid \text { A0A1Q9DG50|A0A1 } \\
\text { Q9DG50_SYMMI }\end{array}$ & 0.5 & $\begin{array}{l}3.0 \mathrm{E}- \\
05\end{array}$ & Putative nucleolar protein 5-1 & O04658 \\
\hline $\begin{array}{c}\operatorname{tr} \mid \text { A0A1Q9CGQ1|A0A1 } \\
\text { Q9CGQ1_SYMMI }\end{array}$ & 0.5 & $\begin{array}{c}9.4 \mathrm{E}- \\
03\end{array}$ & $\begin{array}{c}\text { N-alpha-acetyltransferase } 25 \\
\text { NatB auxiliary subunit }\end{array}$ & Q14CX7 \\
\hline $\begin{array}{c}\operatorname{tr|A0A1Q9EHS0|A0A1~} \\
\text { Q9EHS0_SYMMI }\end{array}$ & 0.5 & $\begin{array}{c}3.7 \mathrm{E}- \\
04\end{array}$ & 40S ribosomal protein $\mathrm{SA}$ & Q8MPF7 \\
\hline $\begin{array}{c}\operatorname{tr}|A 0 A 1 Q 9 B W B 5| A 0 A 1 \\
\text { Q9BWB5_SYMMI }\end{array}$ & 0.5 & $\begin{array}{c}1.8 \mathrm{E}- \\
02\end{array}$ & 40S ribosomal protein $\mathrm{S} 18$ & Q8ISP0 \\
\hline $\begin{array}{c}\operatorname{tr} \mid \text { A0A1Q9CZA7|A0A1 } \\
\text { Q9CZA7_SYMMI }\end{array}$ & 0.5 & $\begin{array}{c}1.3 \mathrm{E}- \\
03\end{array}$ & Elongation factor 2 & Q23716 \\
\hline $\begin{array}{l}\operatorname{tr}|A 0 A 1 Q 9 C P Y 5| A 0 A 1 \\
\text { Q9CPY5_SYMMI }\end{array}$ & 0.5 & $\begin{array}{c}1.0 \mathrm{E}- \\
02\end{array}$ & Tubulin alpha chain & Q71G51 \\
\hline $\begin{array}{l}\operatorname{tr} \mid \text { A0A1Q9DCZ5|A0A1 } \\
\text { Q9DCZ5_SYMMI }\end{array}$ & 0.5 & $\begin{array}{c}5.6 \mathrm{E}- \\
03\end{array}$ & Nucleolar protein 56 & O00567 \\
\hline $\begin{array}{l}\operatorname{tr|A0A1Q9DJS7|A0A1~} \\
\text { Q9DJS7_SYMMI }\end{array}$ & 0.4 & $\begin{array}{c}3.1 \mathrm{E}- \\
04\end{array}$ & $60 S$ ribosomal protein L11 & P0CT77 \\
\hline $\begin{array}{l}\operatorname{tr|A0A1Q9F6G7|A0A1~} \\
\text { Q9F6G7_SYMMI }\end{array}$ & 0.4 & $\begin{array}{c}5.6 \mathrm{E}- \\
03\end{array}$ & $\begin{array}{l}\text { Peptide deformylase 1A, } \\
\text { chloroplastic }\end{array}$ & Q9FUZ0 \\
\hline $\begin{array}{l}\text { tr|A0A1Q9EEJ5|A0A1 } \\
\text { Q9EEJ5_SYMMI }\end{array}$ & -1.0 & $\begin{array}{c}3.8 \mathrm{E}- \\
04\end{array}$ & $\begin{array}{c}\text { Developmentally-regulated G- } \\
\text { protein } 2\end{array}$ & Q9CAI1 \\
\hline $\begin{array}{l}\operatorname{tr|A0A1Q9CRQ6|A0A1~} \\
\text { Q9CRQ6_SYMMI }\end{array}$ & -0.5 & $\begin{array}{c}6.3 \mathrm{E}- \\
03\end{array}$ & $40 S$ ribosomal protein $S 12$ & Q9SMI3 \\
\hline \multicolumn{5}{|l|}{ Protein transport } \\
\hline $\begin{array}{l}\operatorname{tr} \mid \text { A0A1Q9DET6|A0A1 } \\
\text { Q9DET6_SYMMI }\end{array}$ & 0.9 & $\begin{array}{c}6.0 \mathrm{E}- \\
04\end{array}$ & $\begin{array}{l}\text { TBC1 domain family member } \\
\text { 10B }\end{array}$ & Q96CN4 \\
\hline $\begin{array}{l}\operatorname{tr}|\mathrm{A} 0 \mathrm{~A} 1 \mathrm{Q} 9 \mathrm{C} 2 \mathrm{~F} 0| \mathrm{A} 0 \mathrm{~A} 1 \\
\text { Q9C2F0_SYMMI }\end{array}$ & 0.8 & $\begin{array}{c}2.6 \mathrm{E}- \\
02\end{array}$ & GTP-binding protein SAR1A & O04834 \\
\hline $\begin{array}{l}\operatorname{tr|A0A1Q9DJS8|A0A1~} \\
\text { Q9DJS8_SYMMI }\end{array}$ & 0.7 & $\begin{array}{c}1.1 \mathrm{E}- \\
02\end{array}$ & Coatomer subunit alpha- 2 & Q9AUR8 \\
\hline $\begin{array}{l}\operatorname{tr} \mid \text { A0A1Q9EML6|A0A1 } \\
\text { Q9EML6_SYMMI }\end{array}$ & 0.5 & $\begin{array}{c}3.2 \mathrm{E}- \\
03\end{array}$ & $\begin{array}{l}\text { TBC domain-containing } \\
\text { protein C4G8.04 }\end{array}$ & Q09830 \\
\hline $\begin{array}{c}\operatorname{tr}|A 0 A 1 Q 9 C E 92| A 0 A 1 \\
\text { Q9CE92_SYMMI }\end{array}$ & 0.4 & $\begin{array}{c}3.5 \mathrm{E}- \\
03\end{array}$ & Tic20 family protein & Q9ZST8 \\
\hline \multicolumn{5}{|l|}{ Protein modification } \\
\hline $\begin{array}{c}\operatorname{tr|A0A1Q9F796|A0A1Q~} \\
\text { 9F796_SYMMI }\end{array}$ & -1.6 & $\begin{array}{c}2.7 \mathrm{E}- \\
04\end{array}$ & $\begin{array}{l}\text { Putative ubiquitin-conjugating } \\
\text { enzyme E2 }\end{array}$ & Q9C918 \\
\hline $\begin{array}{c}\operatorname{tr|A0A1Q9CKC1|A0A1~} \\
\text { Q9CKC1_SYMMI }\end{array}$ & -1.1 & $\begin{array}{c}1.3 \mathrm{E}- \\
03\end{array}$ & $\begin{array}{c}\text { Bifunctional lysine-specific } \\
\text { demethylase and histidyl- } \\
\text { hydroxylase N }\end{array}$ & Q5ZMM1 \\
\hline \multicolumn{5}{|l|}{ Proteolysis } \\
\hline $\begin{array}{c}\operatorname{tr} \mid \text { A0A1Q9E724|A0A1 } \\
\text { Q9E724_SYMMI }\end{array}$ & 0.9 & $\begin{array}{l}9.1 \mathrm{E}- \\
05\end{array}$ & Ras-related protein $\mathrm{Rab}-7 \mathrm{a}$ & H9BW96 \\
\hline $\begin{array}{c}\operatorname{tr} \mid \text { A0A1Q9DI57|A0A1Q } \\
\text { 9DI57_SYMMI }\end{array}$ & 0.9 & $\begin{array}{c}4.4 \mathrm{E}- \\
03\end{array}$ & Aminopeptidase M1-A & P55786 \\
\hline $\begin{array}{c}\operatorname{tr} \mid \text { A0A1Q9E4W6|A0A1 } \\
\text { Q9E4W6_SYMMI }\end{array}$ & 0.7 & $\begin{array}{l}3.2 \mathrm{E}- \\
04\end{array}$ & Xylem cysteine proteinase 1 & A2XQE8 \\
\hline
\end{tabular}




\begin{tabular}{|c|c|c|c|c|}
\hline $\begin{array}{l}\text { tr|A0A1Q9CZB8|A0A1 } \\
\text { Q9CZB8_SYMMI }\end{array}$ & 0.6 & $\begin{array}{c}2.6 \mathrm{E}- \\
02\end{array}$ & $\begin{array}{l}\text { ATP-dependent Clp protease } \\
\text { ATP-binding subunit ClpA } \\
\text { homolog CD4B, chloroplastic }\end{array}$ & P31542 \\
\hline $\begin{array}{c}\operatorname{tr} \mid \text { A0A1Q9C9W3|A0A1 } \\
\text { Q9C9W3_SYMMI }\end{array}$ & -0.7 & $\begin{array}{c}7.5 \mathrm{E}- \\
03\end{array}$ & Cathepsin B & P10605 \\
\hline \multicolumn{5}{|l|}{ Protein folding } \\
\hline $\begin{array}{l}\operatorname{tr|A0A1Q9E6M5|A0A1~} \\
\text { Q9E6M5_SYMMI }\end{array}$ & 0.6 & $\begin{array}{c}1.7 \mathrm{E}- \\
02\end{array}$ & Prohibitin-1, mitochondrial & O49460 \\
\hline $\begin{array}{l}\operatorname{tr}|A 0 A 1 Q 9 D 7 G 5| A 0 A 1 \\
\text { Q9D7G5_SYMMI }\end{array}$ & 0.4 & $\begin{array}{c}1.6 \mathrm{E}- \\
03\end{array}$ & Luminal-binding protein 5 & P49118 \\
\hline $\begin{array}{l}\operatorname{tr|A0A1Q9CVR8|A0A1~} \\
\text { Q9CVR8_SYMMI }\end{array}$ & -0.9 & $\begin{array}{c}6.3 \mathrm{E}- \\
03\end{array}$ & Peptidylprolyl isomerase & Q7Z895 \\
\hline $\begin{array}{l}\operatorname{tr} \mid \text { A0A1Q9EJH2|A0A1 } \\
\text { Q9EJH2_SYMMI }\end{array}$ & -0.8 & $\begin{array}{c}7.7 \mathrm{E}- \\
04\end{array}$ & $\begin{array}{l}\text { Peptidyl-prolyl cis-trans } \\
\text { isomerase }\end{array}$ & P42693 \\
\hline \multicolumn{5}{|l|}{ TCA cycle } \\
\hline $\begin{array}{l}\operatorname{tr}|A 0 A 1 Q 9 E B 44| A 0 A 1 \\
\text { Q9EB44_SYMMI }\end{array}$ & 0.9 & $\begin{array}{c}1.8 \mathrm{E}- \\
03\end{array}$ & $\begin{array}{l}\text { Phosphoenolpyruvate } \\
\text { carboxylase }\end{array}$ & Q43299 \\
\hline $\begin{array}{l}\operatorname{tr|A0A1Q9ECP6|A0A1~} \\
\text { Q9ECP6_SYMMI }\end{array}$ & 0.8 & $\begin{array}{c}3.7 \mathrm{E}- \\
02\end{array}$ & Citrate synthase & P20115 \\
\hline $\begin{array}{l}\operatorname{tr|A0A1Q9CHP1|A0A1~} \\
\text { Q9CHP1_SYMMI }\end{array}$ & 0.7 & $\begin{array}{c}3.4 \mathrm{E}- \\
02\end{array}$ & $\begin{array}{l}\text { Putative fumarate reductase } \\
\text { (Fragment) }\end{array}$ & O13755 \\
\hline $\begin{array}{c}\operatorname{tr|A0A1Q9F2F5|A0A1~} \\
\text { Q9F2F5_SYMMI }\end{array}$ & 0.5 & $\begin{array}{c}2.0 \mathrm{E}- \\
02\end{array}$ & $\begin{array}{c}\text { Malate dehydrogenase, } \\
\text { mitochondrial }\end{array}$ & Q42686 \\
\hline $\begin{array}{c}\operatorname{tr}|\mathrm{A} 0 \mathrm{~A} 1 \mathrm{Q} 9 \mathrm{CHC} 2| \mathrm{A} 0 \mathrm{~A} 1 \\
\text { Q9CHC2_SYMMI }\end{array}$ & 0.5 & $\begin{array}{c}2.4 \mathrm{E}- \\
03\end{array}$ & Putative fumarate reductase & O13755 \\
\hline $\begin{array}{c}\operatorname{tr}|A 0 A 1 Q 9 C 547| A 0 A 1 \\
\text { Q9C547_SYMMI }\end{array}$ & 0.5 & $\begin{array}{c}2.5 \mathrm{E}- \\
03\end{array}$ & $\begin{array}{l}\text { Malate dehydrogenase, } \\
\text { mitochondrial }\end{array}$ & P00346 \\
\hline $\begin{array}{l}\operatorname{tr}|A 0 A 1 Q 9 E P 32| A 0 A 1 \\
\text { Q9EP32_SYMMI }\end{array}$ & 0.5 & $\begin{array}{c}1.7 \mathrm{E}- \\
03\end{array}$ & $\begin{array}{l}\text { Putative malate:quinone } \\
\text { oxidoreductase }\end{array}$ & C5DAE0 \\
\hline $\begin{array}{c}\operatorname{tr} \mid \text { A0A1Q9C603|A0A1 } \\
\text { Q9C603_SYMMI }\end{array}$ & 0.4 & $\begin{array}{c}6.1 \mathrm{E}- \\
03\end{array}$ & Putative fumarate reductase & O13755 \\
\hline \multicolumn{5}{|l|}{$\begin{array}{c}\text { Lipid metabolism and } \\
\text { transport }\end{array}$} \\
\hline $\begin{array}{c}\text { tr|A0A1Q9E9F5|A0A1 } \\
\text { Q9E9F5_SYMMI }\end{array}$ & 0.9 & $\begin{array}{c}1.3 \mathrm{E}- \\
02\end{array}$ & $\begin{array}{c}\text { Long-chain-fatty-acid--CoA } \\
\text { ligase } 5\end{array}$ & O88813 \\
\hline $\begin{array}{l}\operatorname{tr|A0A1Q9EMQ5|A0A1~} \\
\text { Q9EMQ5_SYMMI }\end{array}$ & 0.7 & $\begin{array}{c}1.9 \mathrm{E}- \\
02\end{array}$ & Extended synaptotagmin-3 & Q5DTI8 \\
\hline $\begin{array}{l}\operatorname{tr} \mid \text { A0A1Q9CKU8|A0A1 } \\
\text { Q9CKU8_SYMMI }\end{array}$ & 0.6 & $\begin{array}{c}2.4 \mathrm{E}- \\
03\end{array}$ & $\begin{array}{c}\text { Peroxisomal bifunctional } \\
\text { enzyme }\end{array}$ & P07896 \\
\hline $\begin{array}{l}\operatorname{tr} \mid \text { A0A1Q9F1G5|A0A1 } \\
\text { Q9F1G5_SYMMI }\end{array}$ & 0.5 & $\begin{array}{c}3.7 \mathrm{E}- \\
03\end{array}$ & $\begin{array}{l}\text { 3-ketoacyl-CoA thiolase A, } \\
\text { peroxisomal }\end{array}$ & Q921H8 \\
\hline $\begin{array}{l}\text { tr|A0A1Q9CVW7|A0A } \\
\text { 1Q9CVW7_SYMMI }\end{array}$ & -0.7 & $\begin{array}{c}2.3 \mathrm{E}- \\
02\end{array}$ & Acyl carrier protein & Q1XDK6 \\
\hline \multicolumn{5}{|l|}{$\begin{array}{l}\text { Carbohydrate } \\
\text { metabolism }\end{array}$} \\
\hline $\begin{array}{l}\operatorname{tr} \mid \text { A0A1Q9CW69|A0A1 } \\
\text { Q9CW69_SYMMI }\end{array}$ & 0.9 & $\begin{array}{c}3.8 \mathrm{E}- \\
02\end{array}$ & $\begin{array}{c}\text { Alpha-glucan water dikinase, } \\
\text { chloroplastic }\end{array}$ & Q8LPT9 \\
\hline $\begin{array}{l}\operatorname{tr|A0A1Q9DEM9|A0A1~} \\
\text { Q9DEM9_SYMMI }\end{array}$ & 0.7 & $\begin{array}{c}7.2 \mathrm{E}- \\
03\end{array}$ & Cyclomaltodextrinase & Q9X2F4 \\
\hline
\end{tabular}




\begin{tabular}{|c|c|c|c|c|}
\hline $\begin{array}{l}\operatorname{tr|A0A1Q9DTX1|A0A1~} \\
\text { Q9DTX1_SYMMI }\end{array}$ & 0.7 & $\begin{array}{c}1.5 \mathrm{E}- \\
02\end{array}$ & $\begin{array}{l}\text { Putative phosphoglucomutase, } \\
\text { cytoplasmic } 1\end{array}$ & O49299 \\
\hline $\begin{array}{l}\operatorname{tr}|A 0 A 1 Q 9 E 0 X 4| A 0 A 1 \\
\text { Q9E0X4_SYMMI }\end{array}$ & 0.7 & $\begin{array}{c}1.7 \mathrm{E}- \\
02\end{array}$ & $\begin{array}{l}\text { 1,4-beta-D-glucan } \\
\text { cellobiohydrolase B }\end{array}$ & Q8NK02 \\
\hline $\begin{array}{l}\operatorname{tr|A0A1Q9EPM6|A0A1~} \\
\text { Q9EPM6_SYMMI }\end{array}$ & 0.6 & $\begin{array}{c}1.4 \mathrm{E}- \\
02\end{array}$ & $\begin{array}{l}\text { 1,3-beta-glucanosyltransferase } \\
\text { GAS2 }\end{array}$ & Q06135 \\
\hline $\begin{array}{l}\operatorname{tr} \mid \text { A0A1Q9E889|A0A1 } \\
\text { Q9E889_SYMMI }\end{array}$ & 0.6 & $\begin{array}{c}2.5 \mathrm{E}- \\
02\end{array}$ & $\begin{array}{l}\text { Alpha-1,4 glucan } \\
\text { phosphorylase }\end{array}$ & Q00766 \\
\hline $\begin{array}{l}\operatorname{tr|A0A1Q9ENK9|A0A1~} \\
\text { Q9ENK9_SYMMI }\end{array}$ & 0.6 & $\begin{array}{c}5.0 \mathrm{E}- \\
04\end{array}$ & $\begin{array}{l}\text { Alpha- } 1,4 \text { glucan } \\
\text { phosphorylase }\end{array}$ & Q00766 \\
\hline $\begin{array}{l}\operatorname{tr} \mid \text { A0A1Q9CBJ7|A0A1 } \\
\text { Q9CBJ7_SYMMI }\end{array}$ & 0.5 & $\begin{array}{c}1.7 \mathrm{E}- \\
05\end{array}$ & $\begin{array}{l}\text { Phosphoenolpyruvate } \\
\text { carboxykinase [ATP] }\end{array}$ & Q75JD5 \\
\hline $\begin{array}{l}\operatorname{tr} \mid \text { A0A1Q9C9H1|A0A1 } \\
\text { Q9C9H1_SYMMI }\end{array}$ & -0.8 & $\begin{array}{c}2.3 \mathrm{E}- \\
02\end{array}$ & $\begin{array}{l}\text { Putative 6- } \\
\text { phosphogluconolactonase } 4, \\
\text { chloroplastic }\end{array}$ & A2YXS5 \\
\hline \multicolumn{5}{|l|}{ Carbohydrate transport } \\
\hline $\begin{array}{l}\operatorname{tr|A0A1Q9EXD2|A0A1~} \\
\text { Q9EXD2_SYMMI }\end{array}$ & 1.1 & $\begin{array}{c}5.2 \mathrm{E}- \\
03\end{array}$ & $\begin{array}{l}\text { NAD }(\mathrm{P}) \text { transhydrogenase } \\
\text { subunit alpha }\end{array}$ & P07001 \\
\hline $\begin{array}{l}\operatorname{tr}|A 0 A 1 Q 9 C R 20| A 0 A 1 \\
\text { Q9CR20_SYMMI }\end{array}$ & 1.1 & $\begin{array}{c}1.8 \mathrm{E}- \\
03\end{array}$ & UDP-galactose translocator 1 & Q6YC49 \\
\hline $\begin{array}{l}\operatorname{tr} \mid \text { A0A1Q9CUK2|A0A1 } \\
\text { Q9CUK2_SYMMI }\end{array}$ & 0.9 & $\begin{array}{c}5.3 \mathrm{E}- \\
03\end{array}$ & $\begin{array}{c}\text { GDP-mannose transporter } \\
\text { GONST5 }\end{array}$ & Q9SFE9 \\
\hline \multicolumn{5}{|l|}{ Glycolysis } \\
\hline $\begin{array}{c}\operatorname{tr|A0A1Q9CPI2|A0A1Q~} \\
\text { 9CPI2_SYMMI }\end{array}$ & 1.2 & $\begin{array}{c}1.6 \mathrm{E}- \\
02\end{array}$ & $\begin{array}{l}\text { Pyruvate dehydrogenase } \\
{[\mathrm{NADP}(+)], \text { mitochondrial }}\end{array}$ & Q94IN5 \\
\hline $\begin{array}{c}\operatorname{tr}|A 0 A 1 Q 9 C J P 3| A 0 A 1 \\
\text { Q9CJP3_SYMMI }\end{array}$ & 0.9 & $\begin{array}{c}2.6 \mathrm{E}- \\
02\end{array}$ & $\begin{array}{l}\text { Pyrophosphate--fructose 6- } \\
\text { phosphate } 1 \text { - } \\
\text { phosphotransferase subunit } \\
\text { alpha } 1 \text { (Fragment) }\end{array}$ & Q9SYP2 \\
\hline $\begin{array}{l}\operatorname{tr} \mid \text { A0A1Q9F5A8|A0A1 } \\
\text { Q9F5A8_SYMMI }\end{array}$ & 0.7 & $\begin{array}{c}6.5 \mathrm{E}- \\
03\end{array}$ & Enolase & Q8IJN7 \\
\hline $\begin{array}{l}\operatorname{tr|A0A1Q9DXW0|A0A~} \\
\text { 1Q9DXW0_SYMMI }\end{array}$ & 0.4 & $\begin{array}{c}2.0 \mathrm{E}- \\
03\end{array}$ & Enolase & Q8IJN7 \\
\hline \multicolumn{5}{|l|}{ Respiration } \\
\hline $\begin{array}{l}\operatorname{tr} \mid \text { A0A1Q9DIC3|A0A1 } \\
\text { Q9DIC3_SYMMI }\end{array}$ & 0.9 & $\begin{array}{c}2.5 \mathrm{E}- \\
02\end{array}$ & $\begin{array}{l}\text { Pyruvate dehydrogenase } \\
\qquad[\mathrm{NADP}(+)]\end{array}$ & Q94IN5 \\
\hline $\begin{array}{l}\operatorname{tr|A0A1Q9DZF2|A0A1~} \\
\text { Q9DZF2_SYMMI }\end{array}$ & 0.7 & $\begin{array}{c}1.7 \mathrm{E}- \\
02\end{array}$ & $\begin{array}{l}\text { Pyruvate dehydrogenase } \\
\text { [NADP }(+)]\end{array}$ & Q968X7 \\
\hline $\begin{array}{l}\operatorname{tr|A0A1Q9DRG7|A0A1~} \\
\text { Q9DRG7_SYMMI }\end{array}$ & -1.7 & $\begin{array}{c}1.2 \mathrm{E}- \\
02\end{array}$ & $\begin{array}{l}\text { ATP synthase subunit epsilon, } \\
\text { mitochondrial }\end{array}$ & Q06450 \\
\hline $\begin{array}{c}\operatorname{tr|A0A1Q9DEF1|A0A1~} \\
\text { Q9DEF1_SYMMI }\end{array}$ & -1.1 & $\begin{array}{l}1.2 \mathrm{E}- \\
04\end{array}$ & $\begin{array}{l}\text { Electron transfer flavoprotein- } \\
\text { ubiquinone oxidoreductase, } \\
\text { mitochondrial }\end{array}$ & Q921G7 \\
\hline \multicolumn{5}{|l|}{$\begin{array}{l}\text { Response to oxidative } \\
\text { stress/ maintenance of } \\
\text { cellular redox } \\
\text { homeostasis }\end{array}$} \\
\hline $\begin{array}{l}\operatorname{tr} \mid \text { A0A1Q9EUX6|A0A1 } \\
\text { Q9EUX6_SYMMI }\end{array}$ & 1.1 & $\begin{array}{l}3.2 \mathrm{E}- \\
04\end{array}$ & $\begin{array}{c}\text { Hematopoietic prostaglandin } \\
\text { D synthase }\end{array}$ & P46436 \\
\hline
\end{tabular}




\begin{tabular}{|c|c|c|c|c|}
\hline $\begin{array}{c}\operatorname{tr|A0A1Q9DK78|A0A1~} \\
\text { Q9DK78_SYMMI }\end{array}$ & 1.0 & $\begin{array}{c}1.7 \mathrm{E}- \\
02\end{array}$ & $\begin{array}{c}\text { Cytochrome c peroxidase, } \\
\text { mitochondrial }\end{array}$ & Q4ING3 \\
\hline $\begin{array}{c}\text { tr|A0A1Q9EXA9|A0A1 } \\
\text { Q9EXA9_SYMMI }\end{array}$ & 1.0 & $\begin{array}{c}4.2 \mathrm{E}- \\
03\end{array}$ & $\begin{array}{l}\mathrm{NAD}(\mathrm{P}) \text { transhydrogenase } \\
\text { mitochondrial }\end{array}$ & Q61941 \\
\hline $\begin{array}{c}\operatorname{tr|A0A1Q9C1P9|A0A1~} \\
\text { Q9C1P9_SYMMI }\end{array}$ & 0.7 & $\begin{array}{c}8.3 \mathrm{E}- \\
03\end{array}$ & Aconitate hydratase 2 & Q8ZRS8 \\
\hline $\begin{array}{c}\operatorname{tr|A0A1Q9CPC6|A0A1~} \\
\text { Q9CPC6_SYMMI }\end{array}$ & 0.6 & $\begin{array}{c}6.3 \mathrm{E}- \\
04\end{array}$ & Cytochrome b5 & P00175 \\
\hline $\begin{array}{l}\operatorname{tr|A0A1Q9ELS3|A0A1~} \\
\text { Q9ELS3_SYMMI }\end{array}$ & -1.3 & $\begin{array}{c}1.3 \mathrm{E}- \\
04\end{array}$ & $\begin{array}{l}\text { Peroxiredoxin-5, } \\
\text { mitochondrial }\end{array}$ & P99029 \\
\hline $\begin{array}{c}\operatorname{tr}|\mathrm{A} 0 \mathrm{~A} 1 \mathrm{Q} 9 \mathrm{E} 4 \mathrm{~J} 3| \mathrm{A} 0 \mathrm{~A} 1 \mathrm{Q} \\
\text { 9E4J3_SYMMI }\end{array}$ & -1.1 & $\begin{array}{l}2.1 \mathrm{E}- \\
05\end{array}$ & $\begin{array}{l}\text { Protein disulfide-isomerase- } \\
\text { like protein EhSep2 }\end{array}$ & Q50KB1 \\
\hline $\begin{array}{c}\operatorname{tr|A0A1Q9CU18|A0A1~} \\
\text { Q9CU18_SYMMI }\end{array}$ & -1.1 & $\begin{array}{c}3.8 \mathrm{E}- \\
04\end{array}$ & Glutaredoxin-C6 & P55142 \\
\hline $\begin{array}{c}\operatorname{tr}|\mathrm{A} 0 \mathrm{~A} 1 \mathrm{Q} 9 \mathrm{C} 7 \mathrm{~J} 4| \mathrm{A} 0 \mathrm{~A} 1 \mathrm{Q} \\
\text { 9C7J4_SYMMI }\end{array}$ & -1.0 & $\begin{array}{c}2.7 \mathrm{E}- \\
02\end{array}$ & Putative nucleoredoxin 1 & O80763 \\
\hline $\begin{array}{c}\operatorname{tr|A0A1Q9ERR3|A0A1~} \\
\text { Q9ERR3_SYMMI }\end{array}$ & -0.9 & $\begin{array}{c}4.4 \mathrm{E}- \\
02\end{array}$ & Prostaglandin E synthase 2 & Q66LN0 \\
\hline $\begin{array}{c}\operatorname{tr|A0A1Q9EV90|A0A1~} \\
\text { Q9EV90_SYMMI }\end{array}$ & -0.9 & $\begin{array}{c}6.0 \mathrm{E}- \\
03\end{array}$ & $\begin{array}{l}\text { Glutaredoxin-related protein } \\
5 \text {, mitochondrial }\end{array}$ & A0A1Q9DIE8 \\
\hline $\begin{array}{c}\operatorname{tr|A0A1Q9DKA2|A0A1~} \\
\text { Q9DKA2_SYMMI }\end{array}$ & -0.8 & $\begin{array}{c}4.1 \mathrm{E}- \\
02\end{array}$ & $\begin{array}{l}\text { Putative NADPH:quinone } \\
\text { oxidoreductase } 1\end{array}$ & Q92R45 \\
\hline $\begin{array}{c}\operatorname{tr|A0A1Q9DV13|A0A1~} \\
\text { Q9DV13_SYMMI }\end{array}$ & -0.6 & $\begin{array}{c}8.6 \mathrm{E}- \\
03\end{array}$ & Superoxide dismutase [Mn] & Q59094 \\
\hline \multicolumn{5}{|l|}{$\begin{array}{c}\text { Transmembrane } \\
\text { transport }\end{array}$} \\
\hline $\begin{array}{l}\operatorname{tr|A0A1Q9CL25|A0A1~} \\
\text { Q9CL25_SYMMI }\end{array}$ & 1.2 & $\begin{array}{c}4.7 \mathrm{E}- \\
02\end{array}$ & Kinesin-like protein $\mathrm{K} 1 \mathrm{~F} 3 \mathrm{~B}$ & Q61771 \\
\hline $\begin{array}{c}\operatorname{tr}|\mathrm{A} 0 \mathrm{~A} 1 \mathrm{Q} 9 \mathrm{C} 4 \mathrm{C} 2| \mathrm{A} 0 \mathrm{~A} 1 \\
\text { Q9C4C2_SYMMI }\end{array}$ & 1.0 & $\begin{array}{c}4.7 \mathrm{E}- \\
03\end{array}$ & $\begin{array}{l}\text { Putative mitochondrial 2- } \\
\text { oxodicarboxylate carrier }\end{array}$ & Q9P3T7 \\
\hline $\begin{array}{c}\operatorname{tr|A0A1Q9CNB5|A0A1~} \\
\text { Q9CNB5_SYMMI }\end{array}$ & 0.5 & $\begin{array}{c}4.1 \mathrm{E}- \\
04\end{array}$ & $\begin{array}{l}\text { Synaptic vesicle 2-related } \\
\text { protein }\end{array}$ & Q940M4 \\
\hline $\begin{array}{c}\operatorname{tr|A0A1Q9C995|A0A1~} \\
\text { Q9C995_SYMMI }\end{array}$ & 0.4 & $\begin{array}{c}6.8 \mathrm{E}- \\
03\end{array}$ & $\begin{array}{l}\text { Multidrug resistance- } \\
\text { associated protein } 1\end{array}$ & Q9C8G9 \\
\hline \multicolumn{5}{|l|}{$\begin{array}{c}\text { Response to nutrient } \\
\text { environment }\end{array}$} \\
\hline $\begin{array}{l}\operatorname{tr|A0A1Q9ESP5|A0A1~} \\
\text { Q9ESP5_SYMMI }\end{array}$ & 1.4 & $\begin{array}{l}2.5 \mathrm{E}- \\
03\end{array}$ & Nitrate reductase & P49102 \\
\hline $\begin{array}{c}\operatorname{tr|A0A1Q9D6C9|A0A1~} \\
\text { Q9D6C9_SYMMI }\end{array}$ & 0.9 & $\begin{array}{c}6.6 \mathrm{E}- \\
03\end{array}$ & Oxalate decarboxylase & A0A1Q9D6C9 \\
\hline $\begin{array}{c}\operatorname{tr} \mid \text { A0A1Q9CW32|A0A1 } \\
\text { Q9CW32_SYMMI }\end{array}$ & -0.7 & $\begin{array}{c}5.5 \mathrm{E}- \\
03\end{array}$ & Purple acid phosphatase 22 & Q8S340 \\
\hline \multicolumn{5}{|l|}{ Photosynthesis } \\
\hline $\begin{array}{l}\operatorname{tr|A0A1Q9CD48|A0A1~} \\
\text { Q9CD48_SYMMI }\end{array}$ & -3.5 & $\begin{array}{c}3.0 \mathrm{E}- \\
02\end{array}$ & Cytochrome c6 & Q3MDW2 \\
\hline $\begin{array}{c}\operatorname{tr|A0A1Q9CFQ0|A0A1~} \\
\text { Q9CFQ0_SYMMI }\end{array}$ & -2.0 & $\begin{array}{c}5.5 \mathrm{E}- \\
05\end{array}$ & $\begin{array}{l}\text { Peridinin-chlorophyll a- } \\
\text { binding protein, chloroplastic }\end{array}$ & P51874 \\
\hline $\begin{array}{c}\operatorname{tr} \mid \text { G9I8S8|G9I8S8_SYM } \\
\text { MI }\end{array}$ & -1.6 & $\begin{array}{c}6.1 \mathrm{E}- \\
05\end{array}$ & $\begin{array}{l}\text { Chloroplast soluble peridinin- } \\
\text { chlorophyll a-binding protein }\end{array}$ & P51874 \\
\hline
\end{tabular}




\begin{tabular}{|c|c|c|c|c|}
\hline $\begin{array}{c}\operatorname{tr|A0A1Q9E3T0|A0A1~} \\
\text { Q9E3T0_SYMMI }\end{array}$ & -1.3 & $\begin{array}{c}2.1 \mathrm{E}- \\
04\end{array}$ & $\begin{array}{l}\text { Photosystem I } 12 \mathrm{kDa} \\
\text { extrinsic protein, chloroplastic }\end{array}$ & Q84XB6 \\
\hline $\begin{array}{l}\operatorname{tr|A0A1Q9D5R1|A0A1~} \\
\text { Q9D5R1_SYMMI }\end{array}$ & -1.1 & $\begin{array}{c}2.8 \mathrm{E}- \\
04\end{array}$ & $\begin{array}{l}\text { Peridinin-chlorophyll a- } \\
\text { binding protein, chloroplastic }\end{array}$ & P51874 \\
\hline $\begin{array}{l}\operatorname{tr|A0A1Q9CID5|A0A1~} \\
\text { Q9CID5_SYMMI }\end{array}$ & -1.1 & $\begin{array}{c}3.7 \mathrm{E}- \\
04\end{array}$ & $\begin{array}{l}\text { Thylakoid lumenal protein, } \\
\text { chloroplastic }\end{array}$ & A0A1Q9CID5 \\
\hline $\begin{array}{c}\operatorname{tr} \mid \text { G9I8R3|G9I8R3_SY } \\
\text { MMI }\end{array}$ & -1.0 & $\begin{array}{c}2.9 \mathrm{E}- \\
05\end{array}$ & $\begin{array}{l}\text { Chloroplast soluble peridinin- } \\
\text { chlorophyll a-binding protein }\end{array}$ & P51874 \\
\hline $\begin{array}{c}\operatorname{tr}|A 0 A 1 Q 9 D 9 H 7| A 0 A 1 \\
\text { Q9D9H7_SYMMI }\end{array}$ & -1.0 & $\begin{array}{c}3.5 \mathrm{E}- \\
03\end{array}$ & Cytochrome c6 & P25935 \\
\hline $\begin{array}{c}\operatorname{tr} \mid \text { G9I8Q7|G9I8Q7_SY } \\
\text { MMI }\end{array}$ & -1.0 & $\begin{array}{c}2.2 \mathrm{E}- \\
04\end{array}$ & $\begin{array}{l}\text { Chloroplast soluble peridinin- } \\
\text { chlorophyll a-binding protein }\end{array}$ & P51874 \\
\hline $\begin{array}{l}\operatorname{tr}|A 0 A 1 Q 9 C B L 0| A 0 A 1 \\
\text { Q9CBL0_SYMMI }\end{array}$ & -0.7 & $\begin{array}{c}6.9 \mathrm{E}- \\
05\end{array}$ & Cytochrome c-550 & А0T0C6 \\
\hline $\begin{array}{c}\operatorname{tr|A0A1Q9BTB7|A0A1~} \\
\text { Q9BTB7_SYMMI }\end{array}$ & -0.5 & $\begin{array}{c}8.0 \mathrm{E}- \\
04\end{array}$ & $\begin{array}{l}\text { Light-harvesting complex } \\
\text { ILH38 protein }\end{array}$ & P08976 \\
\hline $\begin{array}{c}\operatorname{tr} \mid \text { A0A1Q9DSM1|A0A1 } \\
\text { Q9DSM1_SYMMI }\end{array}$ & -0.5 & $\begin{array}{l}1.4 \mathrm{E}- \\
02\end{array}$ & $\begin{array}{l}\text { Fucoxanthin-chlorophyll a-c } \\
\text { binding protein } \mathrm{F} \text {, } \\
\text { chloroplastic }\end{array}$ & Q40300 \\
\hline \multicolumn{5}{|l|}{$\begin{array}{c}\text { Photosynthetic ATP } \\
\text { synthesis }\end{array}$} \\
\hline $\begin{array}{l}\operatorname{tr}|A 0 A 1 Q 9 E G S 5| A 0 A 1 \\
\text { Q9EGS5_SYMMI }\end{array}$ & -0.7 & $\begin{array}{c}8.9 \mathrm{E}- \\
03\end{array}$ & $\begin{array}{l}\text { ATP synthase subunit b, } \\
\text { chloroplastic }\end{array}$ & P193666 \\
\hline $\begin{array}{c}\operatorname{tr} \mid \text { A0A1Q9DKL5|A0A1 } \\
\text { Q9DKL5_SYMMI }\end{array}$ & -0.4 & $\begin{array}{c}1.6 \mathrm{E}- \\
03\end{array}$ & ATP synthase gamma chain & Q06908 \\
\hline \multicolumn{5}{|l|}{ Calvin cycle } \\
\hline $\begin{array}{l}\operatorname{tr|A0A1Q9D916|A0A1~} \\
\text { Q9D916_SYMMI }\end{array}$ & -0.7 & $\begin{array}{c}1.1 \mathrm{E}- \\
02\end{array}$ & Phosphoribulokinase & P26302 \\
\hline $\begin{array}{c}\operatorname{tr} \mid \text { A0A1Q9E3J0|A0A1Q } \\
\text { 9E3J0_SYMMI }\end{array}$ & -0.6 & $\begin{array}{c}1.8 \mathrm{E}- \\
06\end{array}$ & Phosphoglycerate kinase & P91427 \\
\hline $\begin{array}{l}\operatorname{tr} \mid \text { A0A1Q9E616|A0A1 } \\
\text { Q9E616_SYMMI }\end{array}$ & -0.4 & $\begin{array}{c}1.7 \mathrm{E}- \\
03\end{array}$ & $\begin{array}{l}\text { Glyceraldehyde-3-phosphate } \\
\text { dehydrogenase, chloroplastic }\end{array}$ & O09452 \\
\hline \multicolumn{5}{|l|}{ Metal ion binding } \\
\hline $\begin{array}{l}\operatorname{tr} \mid \text { A0A1Q9C8U4|A0A1 } \\
\text { Q9C8U4_SYMMI }\end{array}$ & 1.1 & $\begin{array}{c}6.0 \mathrm{E}- \\
04\end{array}$ & Arylsulfatase B & P50429 \\
\hline $\begin{array}{c}\operatorname{tr}|A 0 A 1 Q 9 F 3 U 5| A 0 A 1 \\
\text { Q9F3U5_SYMMI }\end{array}$ & 0.8 & $\begin{array}{l}2.2 \mathrm{E}- \\
02\end{array}$ & $\begin{array}{c}\text { Peroxisomal NADH } \\
\text { pyrophosphatase NUDT12 }\end{array}$ & Q7NTZ8 \\
\hline $\begin{array}{c}\operatorname{tr} \mid \mathrm{A0A1Q9CHQ4|A0A1} \\
\text { Q9CHQ4_SYMMI }\end{array}$ & -1.0 & $\begin{array}{l}7.9 \mathrm{E}- \\
05\end{array}$ & $\begin{array}{c}\text { Putative ADP-ribosylation } \\
\text { factor GTPase-activating } \\
\text { protein AGD14 }\end{array}$ & Q8RXE7 \\
\hline \multicolumn{5}{|l|}{ ATP binding } \\
\hline $\begin{array}{l}\operatorname{tr|A0A1Q9CRS4|A0A1~} \\
\text { Q9CRS4_SYMMI }\end{array}$ & 0.6 & $\begin{array}{c}2.6 \mathrm{E}- \\
02\end{array}$ & $\begin{array}{l}\text { Serine/threonine-protein } \\
\text { kinase SRPK }\end{array}$ & Q45FA5 \\
\hline $\begin{array}{l}\operatorname{tr} \mid \text { A0A1Q9D1G3|A0A1 } \\
\text { Q9D1G3_SYMMI }\end{array}$ & 0.6 & $\begin{array}{c}2.2 \mathrm{E}- \\
03\end{array}$ & $\begin{array}{c}\text { Dynein heavy chain } 5, \\
\text { axonemal }\end{array}$ & Q8TE73 \\
\hline \multicolumn{5}{|l|}{ Cell division } \\
\hline $\begin{array}{l}\operatorname{tr} \mid \text { A0A1Q9EA60|A0A1 } \\
\text { Q9EA60_SYMMI }\end{array}$ & -0.8 & $\begin{array}{c}4.3 \mathrm{E}- \\
03\end{array}$ & $\begin{array}{l}\text { Serine/threonine-protein } \\
\text { kinase mph1 }\end{array}$ & O94235 \\
\hline
\end{tabular}




\begin{tabular}{|c|c|c|c|c|}
\hline $\begin{array}{c}\operatorname{tr} \mid \text { A0A1Q9C5V1|A0A1 } \\
\text { Q9C5V1_SYMMI }\end{array}$ & -0.8 & $\begin{array}{c}5.1 \mathrm{E}- \\
03\end{array}$ & $\begin{array}{c}\text { Cytoplasmic dynein } 1 \text { heavy } \\
\text { chain } 1\end{array}$ & Q9JHU4 \\
\hline $\begin{array}{l}\operatorname{tr} \mid \text { A0A1Q9ESU8|A0A1 } \\
\text { Q9ESU8_SYMMI }\end{array}$ & 0.5 & $\begin{array}{c}1.1 \mathrm{E}- \\
03\end{array}$ & Tubulin beta chain & P33188 \\
\hline \multicolumn{5}{|l|}{ Membrane component } \\
\hline $\begin{array}{l}\operatorname{tr|A0A1Q9D1M4|A0A1~} \\
\text { Q9D1M4_SYMMI }\end{array}$ & -1.6 & $\begin{array}{c}2.6 \mathrm{E}- \\
03\end{array}$ & Suprabasin & A0A1Q9D1M4 \\
\hline $\begin{array}{l}\operatorname{tr|A0A1Q9ERC6|A0A1~} \\
\text { Q9ERC6_SYMMI }\end{array}$ & -1.4 & $\begin{array}{c}2.7 \mathrm{E}- \\
04\end{array}$ & $\begin{array}{l}\text { Neurofilament medium } \\
\text { polypeptide }\end{array}$ & A0A1Q9ERC6 \\
\hline \multicolumn{5}{|l|}{ Antibiotic biosynthesis } \\
\hline $\begin{array}{c}\operatorname{tr|A0A1Q9C3P7|A0A1~} \\
\text { Q9C3P7_SYMMI }\end{array}$ & 0.6 & $\begin{array}{c}2.7 \mathrm{E}- \\
02\end{array}$ & $\begin{array}{l}\text { Gramicidin } \mathrm{S} \text { biosynthesis } \\
\text { protein GrsT }\end{array}$ & P14686 \\
\hline $\begin{array}{c}\operatorname{tr}|A 0 A 1 Q 9 C H W 8| A 0 A \\
\text { 1Q9CHW8_SYMMI }\end{array}$ & 0.6 & $\begin{array}{c}1.7 \mathrm{E}- \\
02\end{array}$ & $\begin{array}{l}\text { Phthiocerol synthesis } \\
\text { polyketide synthase type }\end{array}$ & Q9ZGI5 \\
\hline \multicolumn{5}{|l|}{$\begin{array}{l}\text { Amino acid } \\
\text { biosynthesis }\end{array}$} \\
\hline $\begin{array}{l}\operatorname{tr}|A 0 A 1 Q 9 C 4 N 6| A 0 A 1 \\
\text { Q9C4N6_SYMMI }\end{array}$ & -0.8 & $\begin{array}{c}1.0 \mathrm{E}- \\
02\end{array}$ & Pentafunctional AR & C5M1X2 \\
\hline $\begin{array}{l}\operatorname{tr|A0A1Q9DDY6|A0A1~} \\
\text { Q9DDY6_SYMMI }\end{array}$ & 0.8 & $\begin{array}{l}2.9 \mathrm{E}- \\
02\end{array}$ & Methionine synthase & Q8DCJ7 \\
\hline \multicolumn{5}{|l|}{ NAD biosynthesis } \\
\hline $\begin{array}{c}\operatorname{tr|A0A1Q9CQ81|A0A1~} \\
\text { Q9CQ81_SYMMI }\end{array}$ & 1.1 & $\begin{array}{c}5.3 \mathrm{E}- \\
03\end{array}$ & $\begin{array}{l}\text { Nicotinamide phosphoribosyl } \\
\text { transferase }\end{array}$ & Q52I78 \\
\hline \multicolumn{5}{|l|}{ Sulfolipid metabolism } \\
\hline $\begin{array}{l}\operatorname{tr|A0A1Q9DDU9|A0A1~} \\
\text { Q9DDU9_SYMMI }\end{array}$ & 0.7 & $\begin{array}{c}1.6 \mathrm{E}- \\
03\end{array}$ & $\begin{array}{l}\text { Putative sulfatase } \\
\text { PB10D8.02c }\end{array}$ & Q9C0V7 \\
\hline
\end{tabular}

Table S3: Differentially abundant proteins between the imbalanced and ambient nutrient regime based on $\log _{2}$ fold change and a student's t-test ( $p$-value presented). Orange $=$ proteins with a greater abundance under an imbalanced nutrient regime relative to the ambient regime. Blue= proteins that a depleted under the imbalanced nutrient regime relative to the ambient regime.

\begin{tabular}{|c|c|c|c|c|}
\hline Genbank accession & $\begin{array}{l}\text { fold } \\
\text { change } \\
\left(\log _{2}\right)\end{array}$ & p-value & Annotation & $\begin{array}{l}\text { Uniprot } \\
\text { accession }\end{array}$ \\
\hline \multicolumn{5}{|l|}{ RNA binding } \\
\hline 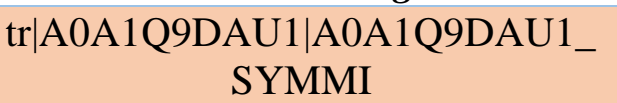 & 0.8 & $3.9 \mathrm{E}-03$ & $\begin{array}{l}\text { ATP-dependent RNA } \\
\text { helicase DED1 }\end{array}$ & Q8TFK8 \\
\hline 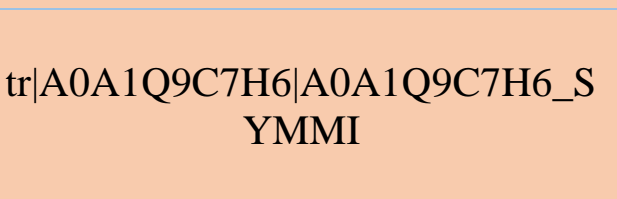 & 0.4 & 4.9E-04 & $\begin{array}{l}116 \mathrm{kDa} \text { U5 small } \\
\text { nuclear } \\
\text { ribonucleoprotein } \\
\text { component }\end{array}$ & Q15029 \\
\hline \multicolumn{5}{|l|}{$\begin{array}{l}\text { mRNA splicing, via the } \\
\text { spliceosome }\end{array}$} \\
\hline $\begin{array}{c}\operatorname{tr}|\mathrm{A} 0 \mathrm{~A} 1 \mathrm{Q} 9 \mathrm{CQG} 3| \mathrm{A} 0 \mathrm{~A} 1 \mathrm{Q} 9 \mathrm{CQG} 3 \\
\text { SYMMI }\end{array}$ & 0.8 & $3.4 \mathrm{E}-03$ & $\begin{array}{l}\text { DEAD-box ATP- } \\
\text { dependent RNA } \\
\text { helicase } 21\end{array}$ & Q53RK8 \\
\hline
\end{tabular}




\begin{tabular}{|c|c|c|c|c|}
\hline 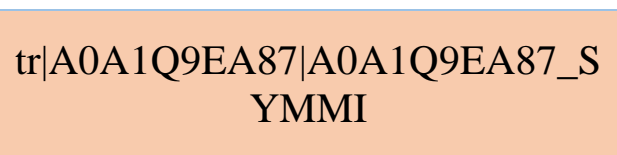 & 0.7 & $5.3 \mathrm{E}-03$ & $\begin{array}{l}\text { DEAD-box ATP- } \\
\text { dependent RNA } \\
\text { helicase } 14\end{array}$ & Q5JKF2 \\
\hline $\begin{array}{c}\operatorname{tr} \mid \text { A0A1Q9EGX9|A0A1Q9EGX9_ } \\
\text { SYMMI }\end{array}$ & 0.5 & $2.6 \mathrm{E}-03$ & $\begin{array}{l}\text { Eukaryotic initiation } \\
\text { factor 4A-III }\end{array}$ & B5DG42 \\
\hline \multicolumn{5}{|l|}{ Translation } \\
\hline $\begin{array}{c}\operatorname{tr|A0A1Q9CHV6|A0A1Q9CHV6~} \\
\text { SYMMI }\end{array}$ & 1.3 & $2.4 \mathrm{E}-03$ & $\begin{array}{l}\text { 30S ribosomal protein } \\
\text { S1-like }\end{array}$ & P38494 \\
\hline 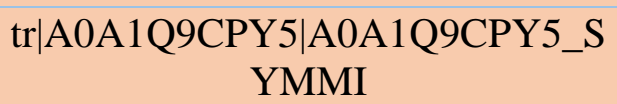 & 0.5 & $5.7 \mathrm{E}-03$ & Tubulin alpha chain & Q71G51 \\
\hline 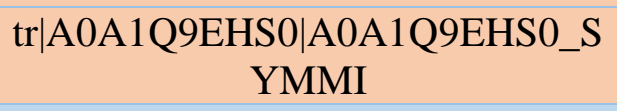 & 0.5 & 2.7E-03 & $\begin{array}{l}\text { 40S ribosomal protein } \\
\text { SA }\end{array}$ & Q8MPF7 \\
\hline $\begin{array}{c}\operatorname{tr}|A 0 A 1 Q 9 C 3 G 1| A 0 A 1 Q 9 C 3 G 1 \_S \\
\text { YMMI }\end{array}$ & -1.0 & $5.9 \mathrm{E}-04$ & $\begin{array}{l}\text { Pentatricopeptide } \\
\text { repeat-containing } \\
\text { protein, chloroplastic }\end{array}$ & Q8GWE0 \\
\hline $\begin{array}{c}\operatorname{tr} \mid \text { A0A1Q9EEJ5|A0A1Q9EEJ5_SY } \\
\text { MMI }\end{array}$ & -0.8 & 3.7E-03 & $\begin{array}{l}\text { Developmentally- } \\
\text { regulated G-protein } 2\end{array}$ & Q9CAI1 \\
\hline \multicolumn{5}{|l|}{ Protein transport } \\
\hline 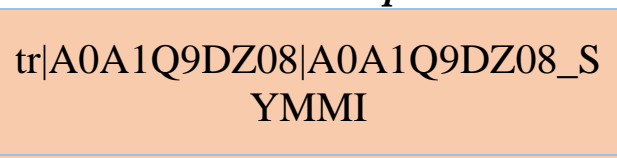 & 0.8 & $1.5 \mathrm{E}-02$ & $\begin{array}{l}\text { Vacuolar protein } \\
\text { sorting-associated } \\
\text { protein } 35 \mathrm{~A}\end{array}$ & Q7X659 \\
\hline $\begin{array}{c}\operatorname{tr} \mid \text { A0A1Q9CE92|A0A1Q9CE92_S } \\
\text { YMMI }\end{array}$ & 0.5 & 7.4E-04 & Tic20 family protein & Q9ZST8 \\
\hline $\begin{array}{c}\operatorname{tr} \mid \text { A0A1Q9EML6|A0A1Q9EML6_ } \\
\text { SYMMI }\end{array}$ & 0.5 & $1.3 \mathrm{E}-03$ & $\begin{array}{l}\text { TBC domain- } \\
\text { containing protein } \\
\text { C4G8.04 }\end{array}$ & Q09830 \\
\hline \multicolumn{5}{|l|}{ Protein folding } \\
\hline 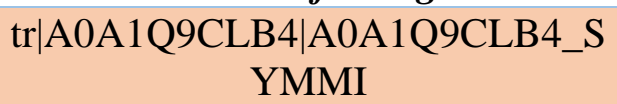 & 1.2 & 8.7E-03 & $\begin{array}{l}\text { Chaperone protein } \\
\text { ClpB }\end{array}$ & Q6N1H2 \\
\hline $\begin{array}{c}\operatorname{tr} \mid \text { A0A1Q9CVR8|A0A1Q9CVR8_ } \\
\text { SYMMI }\end{array}$ & -0.9 & $6.3 \mathrm{E}-03$ & $\begin{array}{l}\text { Peptidylprolyl } \\
\text { isomerase }\end{array}$ & Q38931 \\
\hline \multicolumn{5}{|l|}{ Protein modification } \\
\hline $\begin{array}{c}\operatorname{tr}|A 0 A 1 Q 9 C 1 N 1| A 0 A 1 Q 9 C 1 N 1 \_S \\
\text { YMMI }\end{array}$ & 0.9 & $1.5 \mathrm{E}-02$ & $\begin{array}{c}\text { Putative } \\
\text { glycosyltransferase }\end{array}$ & Q59002 \\
\hline $\begin{array}{c}\operatorname{tr} \mid \text { A0A1Q9CGQ1|A0A1Q9CGQ1_ } \\
\text { SYMMI }\end{array}$ & 0.9 & $2.9 \mathrm{E}-04$ & $\begin{array}{l}\mathrm{N} \text {-alpha- } \\
\text { acetyltransferase } 25, \\
\text { NatB auxiliary subunit }\end{array}$ & Q14CX7 \\
\hline 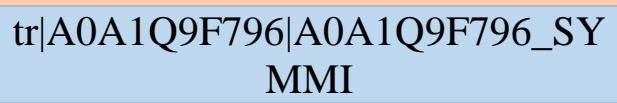 & -1.6 & $2.5 \mathrm{E}-05$ & $\begin{array}{l}\text { Putative ubiquitin- } \\
\text { conjugating enzyme E2 }\end{array}$ & Q9C918 \\
\hline $\begin{array}{c}\operatorname{tr} \mid \text { A0A1Q9CKC1|A0A1Q9CKC1_ } \\
\text { SYMMI }\end{array}$ & -0.7 & $3.0 \mathrm{E}-03$ & $\begin{array}{l}\text { Bifunctional lysine- } \\
\text { specific demethylase } \\
\text { and histidyl- } \\
\text { hydroxylase N }\end{array}$ & Q5ZMM1 \\
\hline \multicolumn{5}{|l|}{ Proteolysis } \\
\hline $\begin{array}{c}\operatorname{tr} \mid \text { A0A1Q9E4W6|A0A1Q9E4W6_ } \\
\text { SYMMI }\end{array}$ & 1.1 & $2.4 \mathrm{E}-04$ & $\begin{array}{l}\text { Xylem cysteine } \\
\text { proteinase } 1\end{array}$ & A2XQE8 \\
\hline $\begin{array}{c}\operatorname{tr}|A 0 A 1 Q 9 D N G 3| A 0 A 1 Q 9 D N G 3 \\
\text { SYMMI }\end{array}$ & 0.9 & $3.8 \mathrm{E}-04$ & $\begin{array}{c}\text { Cathepsin B-like } \\
\text { cysteine proteinase } 6\end{array}$ & P43510 \\
\hline
\end{tabular}




\begin{tabular}{|c|c|c|c|c|}
\hline 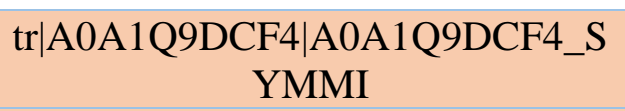 & 0.7 & 4.3E-03 & Oryzain alpha chain & P25776 \\
\hline 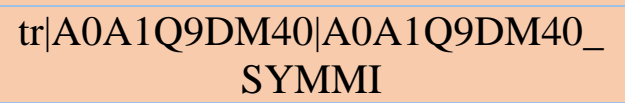 & 0.7 & $4.0 \mathrm{E}-04$ & 14-3-3-like protein & O65352 \\
\hline $\begin{array}{c}\operatorname{tr}|A 0 A 1 Q 9 C V 48| A 0 A 1 Q 9 C V 48 \_S \\
\text { YMMI }\end{array}$ & 0.7 & $4.2 \mathrm{E}-03$ & $\begin{array}{l}\text { Xylem cysteine } \\
\text { proteinase } 1\end{array}$ & O65493 \\
\hline \multicolumn{5}{|l|}{ Photosystems } \\
\hline 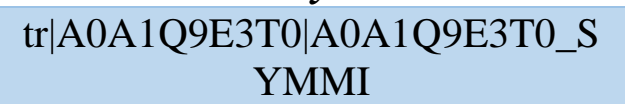 & -1.5 & $1.0 \mathrm{E}-02$ & $\begin{array}{l}\text { Photosystem II } 12 \mathrm{kDa} \\
\text { extrinsic protein }\end{array}$ & Q84XB6 \\
\hline 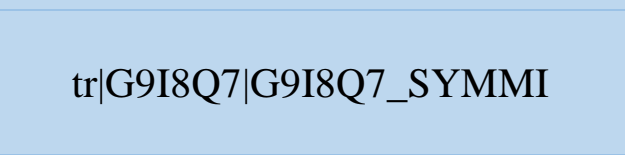 & -0.9 & 7.6E-04 & $\begin{array}{l}\text { Chloroplast soluble } \\
\text { peridinin-chlorophyll } \\
\text { a-binding protein }\end{array}$ & P51874 \\
\hline 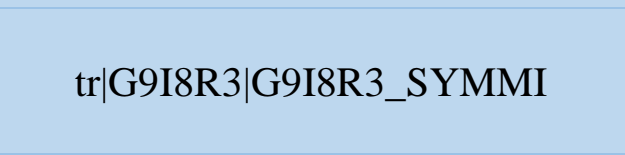 & -0.8 & $5.1 \mathrm{E}-04$ & $\begin{array}{l}\text { Chloroplast soluble } \\
\text { peridinin-chlorophyll } \\
\text { a-binding protein }\end{array}$ & P51874 \\
\hline $\begin{array}{c}\operatorname{tr}|A 0 A 1 Q 9 D 5 R 1| A 0 A 1 Q 9 D 5 R 1 \_S \\
\text { YMMI }\end{array}$ & -0.8 & 2.7E-03 & $\begin{array}{l}\text { Peridinin-chlorophyll } \\
\text { a-binding protein, } \\
\text { chloroplastic }\end{array}$ & P51874 \\
\hline 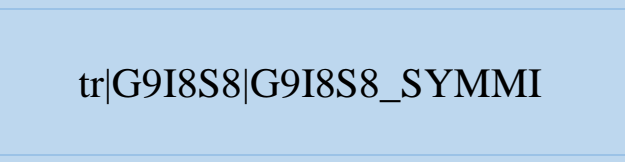 & -0.8 & $1.7 \mathrm{E}-02$ & $\begin{array}{l}\text { Chloroplast soluble } \\
\text { peridinin-chlorophyll } \\
\text { a-binding protein }\end{array}$ & P51874 \\
\hline 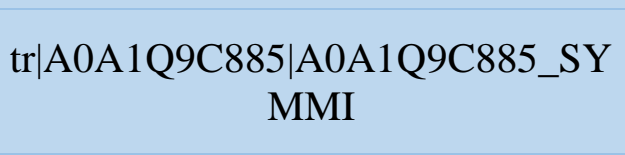 & -0.6 & $2.4 \mathrm{E}-03$ & $\begin{array}{l}\text { Caroteno-chlorophyll } \\
\text { a-c-binding protein } \\
\text { (Fragment) }\end{array}$ & P55738 \\
\hline $\begin{array}{c}\operatorname{tr}|A 0 A 1 Q 9 B T B 7| A 0 A 1 Q 9 B T B 7 \_S \\
\text { YMMI }\end{array}$ & -0.6 & $2.2 \mathrm{E}-04$ & $\begin{array}{l}\text { Light-harvesting } \\
\text { complex }\end{array}$ & P08976 \\
\hline \multicolumn{5}{|l|}{ Photosynthetic ATP synthesis } \\
\hline $\begin{array}{c}\operatorname{tr}|A 0 A 1 Q 9 E G S 5| A 0 A 1 Q 9 E G S 5 \_S \\
\text { YMMI }\end{array}$ & -1.0 & $1.5 \mathrm{E}-02$ & $\begin{array}{l}\text { ATP synthase subunit } \\
\text { b, chloroplastic }\end{array}$ & P193666 \\
\hline $\begin{array}{c}\operatorname{tr|A0A1Q9DKL5|A0A1Q9DKL5~} \\
\text { SYMMI }\end{array}$ & -0.5 & 3.7E-03 & $\begin{array}{l}\text { ATP synthase gamma } \\
\text { chain }\end{array}$ & Q06908 \\
\hline \multicolumn{5}{|l|}{ Calvin cycle } \\
\hline 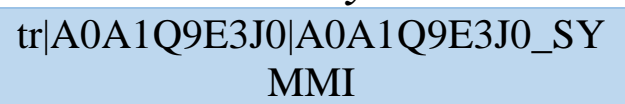 & -0.6 & 7.3E-06 & $\begin{array}{l}\text { Phosphoglycerate } \\
\text { kinase }\end{array}$ & P91427 \\
\hline 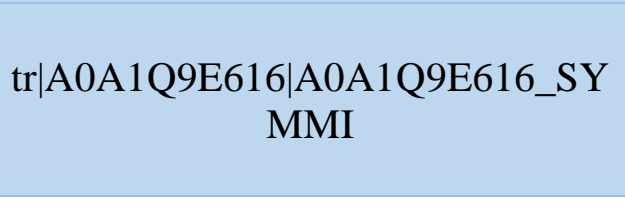 & -0.5 & $1.1 \mathrm{E}-03$ & $\begin{array}{l}\text { Glyceraldehyde-3- } \\
\text { phosphate } \\
\text { dehydrogenase, } \\
\text { chloroplastic }\end{array}$ & O09452 \\
\hline \multicolumn{5}{|l|}{ Respiratory electron transport } \\
\hline $\begin{array}{c}\operatorname{tr}|A 0 A 1 Q 9 D E F 1| A 0 A 1 Q 9 D E F 1 \_S \\
\text { YMMI }\end{array}$ & -1.0 & $3.3 \mathrm{E}-03$ & $\begin{array}{l}\text { Electron transfer } \\
\text { flavoprotein- } \\
\text { ubiquinone } \\
\text { oxidoreductase, } \\
\text { mitochondrial }\end{array}$ & Q921G7 \\
\hline \multicolumn{5}{|l|}{ Carbohydrate metabolism } \\
\hline $\begin{array}{c}\operatorname{tr}|\mathrm{A} 0 \mathrm{~A} 1 \mathrm{Q} 9 \mathrm{CW} 69| \mathrm{A} 0 \mathrm{~A} 1 \mathrm{Q} 9 \mathrm{CW69} \\
\text { SYMMI }\end{array}$ & 1.2 & $4.2 \mathrm{E}-03$ & $\begin{array}{l}\text { Alpha-glucan water } \\
\text { dikinase }\end{array}$ & Q8LPT9 \\
\hline 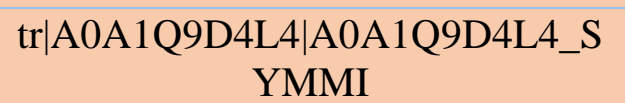 & 1.1 & 3.7E-04 & Alpha-galactosidase & P51569 \\
\hline
\end{tabular}




\begin{tabular}{|c|c|c|c|c|}
\hline $\begin{array}{c}\operatorname{tr}|\mathrm{A} 0 \mathrm{~A} 1 \mathrm{Q} 9 \mathrm{ENK} 9| \mathrm{A} 0 \mathrm{~A} 1 \mathrm{Q} 9 \mathrm{ENK} 9 \\
\text { SYMMI }\end{array}$ & 0.6 & 7.9E-04 & $\begin{array}{l}\text { Alpha-1,4 glucan } \\
\text { phosphorylase }\end{array}$ & Q00766 \\
\hline $\begin{array}{c}\text { tr|A0A1Q9DTP6|A0A1Q9DTP6_S } \\
\text { YMMI }\end{array}$ & 0.6 & $2.1 \mathrm{E}-05$ & $\begin{array}{l}\text { Platelet glycoprotein Ib } \\
\text { alpha chain }\end{array}$ & G4MM92 \\
\hline $\begin{array}{c}\operatorname{tr} \mid \text { A0A1Q9EPM6|A0A1Q9EPM6_ } \\
\text { SYMMI }\end{array}$ & 0.6 & $3.3 \mathrm{E}-03$ & $\begin{array}{c}\text { 1,3-beta- } \\
\text { glucanosyltransferase } \\
\text { GAS2 }\end{array}$ & Q06135 \\
\hline \multicolumn{5}{|l|}{ Glycolysis } \\
\hline 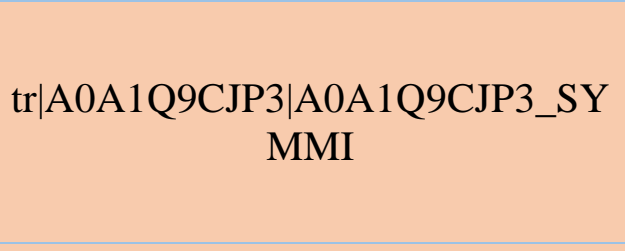 & 1.2 & 5.0E-04 & $\begin{array}{l}\text { Pyrophosphate-- } \\
\text { fructose 6-phosphate 1- } \\
\text { phosphotransferase } \\
\text { subunit alpha } 1 \\
\text { (Fragment) }\end{array}$ & Q9SYP2 \\
\hline 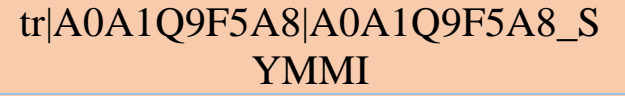 & 1.0 & $1.4 \mathrm{E}-04$ & Enolase & Q8IJN7 \\
\hline 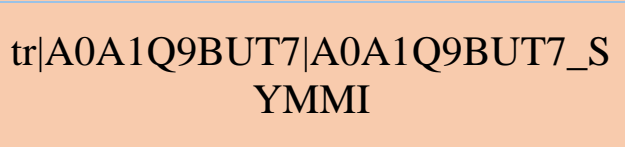 & 0.8 & 9.9E-04 & $\begin{array}{l}\text { Fructose-bisphosphate } \\
\text { aldolase class } 2 \\
\text { (Fragment) }\end{array}$ & O52402 \\
\hline \multicolumn{5}{|l|}{ Fatty acid beta oxidation } \\
\hline 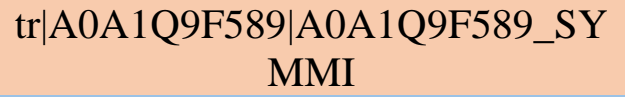 & 1.4 & $5.0 \mathrm{E}-03$ & Aconitate hydratase 2 & Q8ZRS8 \\
\hline 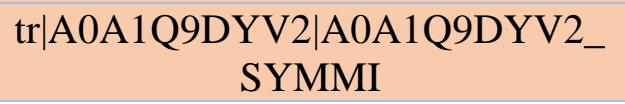 & 0.5 & $1.6 \mathrm{E}-03$ & $\begin{array}{l}\text { Long chain acyl-CoA } \\
\text { synthetase } 8\end{array}$ & Q9SJD4 \\
\hline \multicolumn{5}{|l|}{ TCA cycle } \\
\hline 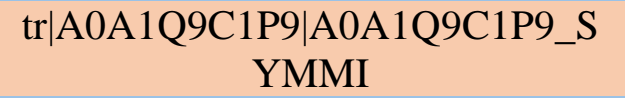 & 0.7 & $4.0 \mathrm{E}-03$ & Aconitate hydratase 2 & Q8ZRS8 \\
\hline \multicolumn{5}{|l|}{ Lipid transport } \\
\hline $\begin{array}{c}\operatorname{tr|A0A1Q9EMQ5|A0A1Q9EMQ5~} \\
\text { SYMMI }\end{array}$ & 1.1 & $1.0 \mathrm{E}-04$ & $\begin{array}{c}\text { Extended } \\
\text { synaptotagmin-3 }\end{array}$ & Q5DTI8 \\
\hline \multicolumn{5}{|l|}{$\begin{array}{c}\text { Response to oxidative stress/ } \\
\text { maintenance of cellular redox } \\
\text { homeostasis }\end{array}$} \\
\hline 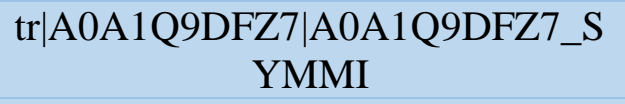 & -1.6 & $6.9 \mathrm{E}-03$ & Flavodoxin & P14070 \\
\hline 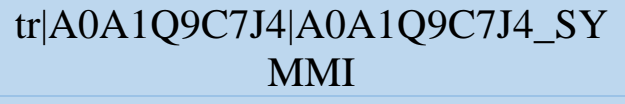 & -1.1 & $1.3 \mathrm{E}-02$ & $\begin{array}{c}\text { Putative nucleoredoxin } \\
1\end{array}$ & O80763 \\
\hline 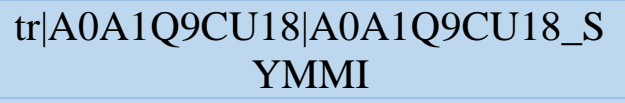 & -0.9 & 9.4E-03 & Glutaredoxin-C6 & P55142 \\
\hline $\begin{array}{c}\operatorname{tr}|A 0 A 1 Q 9 E L S 3| A 0 A 1 Q 9 E L S 3 \_S \\
\text { YMMI }\end{array}$ & -0.9 & $5.8 \mathrm{E}-03$ & $\begin{array}{l}\text { Peroxiredoxin-5, } \\
\text { mitochondrial }\end{array}$ & P99029 \\
\hline 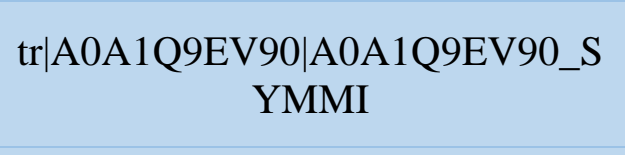 & -0.9 & $1.2 \mathrm{E}-02$ & $\begin{array}{l}\text { Glutaredoxin-related } \\
\text { protein } 5, \\
\text { mitochondrial }\end{array}$ & $\begin{array}{l}\text { A0A1Q9D } \\
\text { IE8 }\end{array}$ \\
\hline $\begin{array}{c}\operatorname{tr}|A 0 A 1 Q 9 E 4 J 3| A 0 A 1 Q 9 E 4 J 3 \_S Y \\
\text { MMI }\end{array}$ & -0.9 & $1.3 \mathrm{E}-03$ & $\begin{array}{l}\text { Protein disulfide- } \\
\text { isomerase-like protein } \\
\text { EhSep2 }\end{array}$ & Q50KB1 \\
\hline $\begin{array}{c}\operatorname{tr}|A 0 A 1 Q 9 D K 78| A 0 A 1 Q 9 D K 78 \_S \\
\text { YMMI }\end{array}$ & 1.0 & $5.2 \mathrm{E}-03$ & $\begin{array}{l}\text { Cytochrome c } \\
\text { peroxidase, } \\
\text { mitochondrial }\end{array}$ & Q4ING3 \\
\hline
\end{tabular}




\begin{tabular}{|c|c|c|c|c|}
\hline $\begin{array}{l}\operatorname{tr|A0A1Q9CAM9|A0A1Q9CAM9~} \\
\text { SYMMI }\end{array}$ & 0.8 & $5.7 \mathrm{E}-03$ & $\begin{array}{l}\text { Putative rhamnose } \\
\text { biosynthetic enzyme } 1\end{array}$ & Q9SYM5 \\
\hline 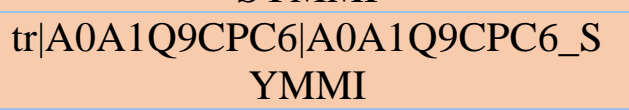 & 0.7 & $2.1 \mathrm{E}-05$ & Cytochrome b5 & P00175 \\
\hline $\begin{array}{c}\operatorname{tr}|A 0 A 1 Q 9 D W 66| A 0 A 1 Q 9 D W 66 \\
\text { SYMMI }\end{array}$ & 0.5 & $1.6 \mathrm{E}-03$ & $\begin{array}{l}\text { Putative L-ascorbate } \\
\text { peroxidase } 7, \\
\text { chloroplastic }\end{array}$ & Q69SV0 \\
\hline \multicolumn{5}{|l|}{ ATPase activity } \\
\hline $\begin{array}{l}\text { tr|A0A1Q9D817|A0A1Q9D817_S } \\
\text { YMMI }\end{array}$ & 0.7 & $4.2 \mathrm{E}-03$ & $\begin{array}{l}\text { Plasma membrane } \\
\text { ATPase }\end{array}$ & Q58623 \\
\hline 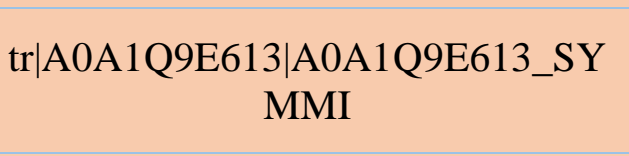 & 0.6 & $9.9 \mathrm{E}-03$ & $\begin{array}{l}\text { ATP-binding cassette } \\
\text { sub-family B member } \\
6, \text { mitochondrial }\end{array}$ & Q08D64 \\
\hline $\begin{array}{l}\text { tr|A0A1Q9D1G3|A0A1Q9D1G3_S } \\
\text { YMMI }\end{array}$ & 0.6 & $8.0 \mathrm{E}-04$ & $\begin{array}{c}\text { Dynein heavy chain } 5, \\
\text { axonemal }\end{array}$ & Q8TE73 \\
\hline $\begin{array}{c}\text { tr|A0A1Q9DT94|A0A1Q9DT94_S } \\
\text { YMMI }\end{array}$ & 0.5 & $3.0 \mathrm{E}-03$ & $\begin{array}{l}\text { Putative V-type proton } \\
\text { ATPase subunit } d\end{array}$ & Q8RU33 \\
\hline \multicolumn{5}{|l|}{ Response to nutrient environment } \\
\hline $\begin{array}{l}\text { tr|A0A1Q9D6C9|A0A1Q9D6C9_S } \\
\text { YMMI }\end{array}$ & 0.8 & $9.0 \mathrm{E}-03$ & Oxalate decarboxylase & $\begin{array}{l}\text { A0A1Q9D } \\
6 \text { C } 9\end{array}$ \\
\hline $\begin{array}{l}\text { tr|A0A1Q9E3K5|A0A1Q9E3K5_S } \\
\text { YMMI }\end{array}$ & 0.7 & 7.4E-03 & $\begin{array}{l}\text { Type- } 3 \text { glutamine } \\
\text { synthetase }\end{array}$ & Q54WR9 \\
\hline $\begin{array}{l}\text { tr|A0A1Q9CW32|A0A1Q9CW32_ } \\
\text { SYMMI }\end{array}$ & -1.0 & $2.7 \mathrm{E}-03$ & $\begin{array}{l}\text { Purple acid } \\
\text { phosphatase } 22\end{array}$ & Q8S340 \\
\hline \multicolumn{5}{|l|}{ Cell division } \\
\hline $\begin{array}{l}\text { tr|A0A1Q9DA17|A0A1Q9DA17_S } \\
\text { YMMI }\end{array}$ & 0.7 & $3.9 \mathrm{E}-05$ & Actin & P53476 \\
\hline 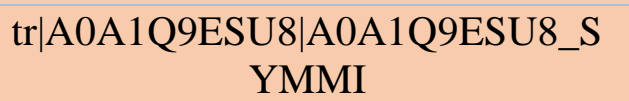 & 0.5 & 3.6E-04 & Tubulin beta chain & P33188 \\
\hline $\begin{array}{l}\text { tr|A0A1Q9EA60|A0A1Q9EA60_S } \\
\text { YMMI }\end{array}$ & -0.8 & $9.0 \mathrm{E}-03$ & $\begin{array}{l}\text { Serine/threonine- } \\
\text { protein kinase mph1 }\end{array}$ & O94235 \\
\hline \multicolumn{5}{|l|}{ Metal binding } \\
\hline 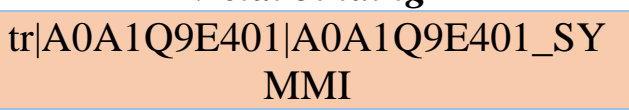 & 1.1 & 8.0E-04 & $\begin{array}{l}\text { Selenium-binding } \\
\text { protein } 1\end{array}$ & Q569D5 \\
\hline $\begin{array}{l}\text { tr|A0A1Q9CRS4|A0A1Q9CRS4_S } \\
\text { YMMI }\end{array}$ & 1.0 & $1.8 \mathrm{E}-04$ & Pumilio-like 3 & $\begin{array}{l}\text { A0A1Q9C } \\
\text { RS4 }\end{array}$ \\
\hline \multicolumn{5}{|l|}{ Cellar signalling } \\
\hline $\begin{array}{c}\text { tr|A0A1Q9C6V5|A0A1Q9C6V5_S } \\
\text { YMMI }\end{array}$ & 0.8 & $7.0 \mathrm{E}-03$ & Arylsulfatase B & P33727 \\
\hline $\begin{array}{c}\operatorname{tr|A0A088MHG6|A0A088MHG6~} \\
\text { SYMMI }\end{array}$ & 0.5 & $7.5 \mathrm{E}-04$ & $\begin{array}{l}\text { Guanine nucleotide } \\
\text { binding protein beta } \\
\text { subunit-like protein }\end{array}$ & $\mathrm{O} 42248$ \\
\hline 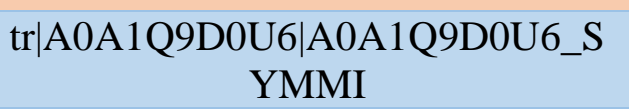 & -1.2 & $6.3 \mathrm{E}-03$ & Calmodulin-1 & P06787 \\
\hline \multicolumn{5}{|l|}{ Alternate oxidase activity } \\
\hline 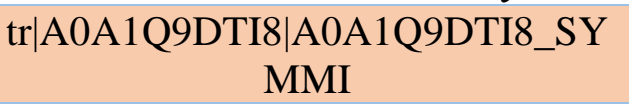 & 0.6 & $4.6 \mathrm{E}-03$ & $\begin{array}{l}\text { Ubiquinol oxidase 1a, } \\
\text { mitochondrial }\end{array}$ & Q39219 \\
\hline
\end{tabular}




\begin{tabular}{|ccccc|}
\hline $\begin{array}{c}\text { tr|A0A1Q9CWR4|A0A1Q9CWR4_ } \\
\text { SYMMI }\end{array}$ & 0.6 & $1.0 \mathrm{E}-02$ & $\begin{array}{c}\text { Violaxanthin de- } \\
\text { epoxidase, } \\
\text { chloroplastic }\end{array}$ & Q40251 \\
\hline $\begin{array}{c}\text { Chlorophyll biosynthesis } \\
\text { tr|A0A1Q9CGJ6|A0A1Q9CGJ6_S } \\
\text { YMMI }\end{array}$ & 0.7 & $2.4 \mathrm{E}-03$ & $\begin{array}{c}\text { Geranylgeranyl } \\
\text { diphosphate reductase, } \\
\text { chloroplastic }\end{array}$ & Q9ZS34 \\
\hline $\begin{array}{c}\text { Sulpholipid metabolic process } \\
\text { tr|A0A1Q9DDU90A1Q9DDU9_ } \\
\text { SYMMI }\end{array}$ & 1.0 & 7.0E-06 & $\begin{array}{c}\text { Putative sulfatase } \\
\text { PB10D8.02c }\end{array}$ & Q9C0V7 \\
\hline $\begin{array}{c}\text { NAD+ biosynthesis } \\
\text { tr|A0A1Q9CQ81|A0A1Q9CQ81_S } \\
\text { YMMI }\end{array}$ & 1.3 & 3.9E-04 & $\begin{array}{c}\text { Nicotinamide } \\
\text { phosphoribosyl } \\
\text { transferase }\end{array}$ & Q52I78 \\
\hline
\end{tabular}

